NBSIR 77-1300

\title{
A Computer-Assisted Evaluation of the Thermochemical Data of the Compounds of Thorium
}

D. D. Wagman, R. H. Schumm and V. B. Parker

Physical Chemistry Division

Institute for Materials Research

National Bureau of Standards

Washington, D.C. 20234

August 1977

n-amserst for

QC onal Atomic Energy Agency

.456 i Standard Reference Data, NBS

no. $77-1300$

1977 


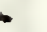




\section{A COMPUTER-ASSISTED EVALUATION OF THE THERMOCHEMICAL DATA OF THE COMPOUNDS OF THORIUM}

D. D. Wagman, R. H. Schumm and V. B. Parker

Physical Chemistry Division Institute for Materials Research National Bureau of Standards

Washington, D.C. 20234

August 1977

Prepared for

International Atomic Energy Agency and

Office of Standard Reference Data, NBS

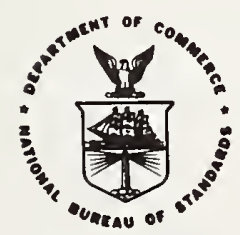

U.S. DEPARTMENT OF COMMERCE, Juanita M. Kreps, Secretary Dr. Sidney Harman, Under Secretary Jordan J. Baruch, Assistant Secretary for Science and Technology NATIONAL BUREAU OF STANDARDS, Ernest Ambler, Acting Director 



\section{TABLE OF CONTENTS}

I. Genera 1 Description

1. Introduction 2

2. Explanation of the Contents of Tables 3

3. Unit of Energy and Fundamental Constants 8

4. Internal Consistency of the Tables 9

5. Arrangement of the Tables 11

II. Tables of Values for $\Delta H f^{\circ}, \triangle G f^{\circ}$ and $S^{\circ}$ at $298.15 \mathrm{~K}$ for the Compounds of Thorium and the Reaction Catalog

III. Bibliography

IV. Appendix: Therma 1 Functions for Thorium Compounds A-1

$\begin{array}{lr}\text { Th }(\mathrm{c}, 1) & \mathrm{A}-6 \\ \mathrm{Th}(\mathrm{g}) & \mathrm{A}-7 \\ \mathrm{ThO}_{(\mathrm{g})} & \mathrm{A}-8 \\ \mathrm{ThO}_{2}(\mathrm{c}) & \mathrm{A}-9 \\ \mathrm{ThO}_{2}(\mathrm{~g}) & \mathrm{A}-10 \\ \mathrm{ThF}_{(\mathrm{g})} & \mathrm{A}-11 \\ \mathrm{ThF}_{2}(\mathrm{~g}) & \mathrm{A}-12 \\ \mathrm{ThF}_{3}(\mathrm{~g}) & \mathrm{A}-13 \\ \mathrm{ThF}_{4}(\mathrm{c}, 1) & \mathrm{A}-14 \\ \mathrm{ThF}_{4}(\mathrm{~g}) & \mathrm{A}-15 \\ \mathrm{ThCl}_{(\mathrm{g})} & \mathrm{A}-16 \\ \mathrm{ThCl}_{2}(\mathrm{~g}) & \mathrm{A}-17 \\ \end{array}$




$\begin{array}{lc}\operatorname{ThCl}_{3}(\mathrm{~g}) & \mathrm{A}-18 \\ \operatorname{ThCl}_{4}(\mathrm{c}, 1) & \mathrm{A}-19 \\ \operatorname{ThCl}_{4}(\mathrm{~g}) & \mathrm{A}-20 \\ \operatorname{ThBr}(\mathrm{g}) & \mathrm{A}-21 \\ \operatorname{ThBr}_{2}(\mathrm{~g}) & \mathrm{A}-22 \\ \operatorname{ThBr}_{3}(\mathrm{~g}) & \mathrm{A}-23 \\ \operatorname{ThBr}_{4}(\mathrm{c}, 1) & \mathrm{A}-24 \\ \operatorname{ThBr}_{4}(\mathrm{~g}) & \mathrm{A}-25 \\ \operatorname{ThI}_{(\mathrm{g})} & \mathrm{A}-26 \\ \operatorname{ThI}_{2}(\mathrm{~g}) & \mathrm{A}-27 \\ \operatorname{ThI}_{3}(\mathrm{~g}) & \mathrm{A}-28 \\ \operatorname{ThI}_{4}(\mathrm{c}, 1) & \mathrm{A}-29\end{array}$


A Computer-Assisted Evaluation of the Thermochemical Data of the Compounds of Thorium

by D. Wagman, R. Schumm, and V. B. Parker

Abstract

Selected values are given for the thermochemical properties of the compounds of thorium. They are obtained from a computer-assisted least sums-least squares approach to the evaluation of thermodynamic data networks. The properties given, where data are available, are enthalpy of formation, Gibbs energy of formation, and entropy at 298.15 K $\left(\Delta H f^{\circ}(298), \Delta G f^{\circ}(298)\right.$, and $\left.S^{\circ}(298)\right)$. The values are consistent with the CODATA Key Values for The rmodynamics. The reaction catalog from which this self consistent set of values is generated is given with a statistical analysis. Some thermal functions are also given, as well as detailed comments when necessary.

Keywords: Data evaluation; enthalpy; entropy; Gibbs energy; thermochemical data networks; thermochemical tables; thorium compounds. 


\section{Introduction}

This report presents a computer-assisted comprehensive evaluation of the thermochemical properties of compounds of thorium at $298 \mathrm{~K}$ that is consistent with the CODATA Key Values for Thermodynamics [75COD] ${ }^{\mathrm{a}}$. A combined least sums-least squares technique previously described by Garvin et al [76GAR/PAR] has been used. This computer-based evaluation is an extension of the CATCH program developed by J. B. Pedley, University of Sussex who used linear least squares analysis on enthalpy measurements [69GU/PED].

Thorium represents the first complete analysis of an element and its compounds to be done by this least sums-least squares approach to the evaluation of thermodynamic data networks. A self consistent set of values for $\Delta \mathrm{Hf}^{\circ}, \triangle \mathrm{Gf} \mathrm{f}^{\circ}$, and $\mathrm{S}^{\circ}$ is generated from the given reaction catalog. A detailed analysis of the values leads us to believe that they are as good as and as reliable as those which would be obtained in the traditional sequential method. Comparisons of the performance of the computer-assisted method used here versus the traditional method are given in [76GAR/PAR].

A major advantage of this method is the rapid updating that is possible when new data become available or if the interpretation of the existing data must be revised. Another advantage of this method is that a translation from one scale of auxiliary values (CODATA here) to the NBS Technical Note 270 Scale or some other scale can be made readily.

a References are given in a chronologically ordered bibliography at the end of the report. They are cited in the text in coded form indicating year of publication and the first two authors. 
This report provides the following data: the final selceted values for $\Delta H f^{\circ}, \Delta G f^{\circ}$ and $S^{\circ}$ for the conpounds considered and their predicted reliabilities, the values used for auxiliary compounds, a catalog of the measurements upon which the solution is based, some newly developed thermal functions and, where, needed, comments on the interpretation of the data.

The values for properties of auxiliary cornpounds are either CODATA selections [75COD] or compatible with them [75PAR/WAG]. The reaction catalog (following the selected values), from which the solution is derived, contains the set of experimental enthalpy, Gibbs energy and entropy changes at $298 \mathrm{~K}$ as we11 as entropies at 298 K from low temperature Cp measurements or statistica 1 calculations. It also contains for each reaction the initial assigned uncertainty, the final weighting factor, the residual (i.e., the observed minus the predicted value), the standard deviation of the predicted value (95 per cent confidence leve1), a coded citation to the Bibliography, and, occasionally, brief comments on corrections made to or interpretation of individual measurements.

User: of tho tables and reaction catalog are invited to comment on the selections, correct errors and bring new measurements to our attention.

\section{Explanation of the Contents of the Tables}

'I'he following material provides definitions and conventions used in the tables. 


\subsection{Chemical Formulae and Physical States}

The tables were reproduced from computer printout in which only capital (upper case) letters are available. Normal one-line chemical formulae are used, with the following modifications:

- Subscripts (counts of atoms) and superscripts (charge) are printed on line: $\mathrm{TH}^{\prime} \mathrm{CL}^{\prime} 4=\mathrm{ThCl}_{4} ; \mathrm{TH}^{\prime} \mathrm{CL} \mathrm{L}^{\prime}+3=\mathrm{ThCl}^{3+}$

- The apostrophe' appears after each letter in a chemical symbol that would normally be written in lower case: TH'N = ThN

- The centered dot, used in hydrates and minerals, is shown as a colon: $\mathrm{TH}^{\prime} \mathrm{F} 4: 2.5 \mathrm{H} 2 \mathrm{O}=\mathrm{ThF}_{4} \cdot 2.5 \mathrm{H}_{2} \mathrm{O}$

- The physical state of the substance is appended to the chemical formula in parentheses: $\mathrm{TH}^{\prime} \mathrm{CL} / 4(\mathrm{C})=\mathrm{ThCl}_{4}$, crystaline Conventions with respect to physical state are given in Table A.

\subsection{Definition of Symbols for Thermochemical Properties}

The headings used in the tabulated Thermochemical Values have the following meanings:

DELTA HF $=\Delta \mathrm{Hf}^{\circ}$, standard enthalpy of formation at $298.15 \mathrm{~K}$; DELTA $G F=\triangle G F^{\circ}$, standard Gibbs energy (formerly free energy), $G$, of formation at 298.15 K;

+/- SIGMA = Predicted reliability, "one sigma" (see paragraph 4.1)

In the reaction catalog the heading, PROP. MEAS. refers to $\triangle H$, $\Delta G$, and $\Delta S$ at $298 \mathrm{~K}$ for the process given; the symbols used are $H, G$, and S; the symbol S means S $_{298}$ when it refers to a "process" written without reactants:

$$
=\mathrm{TH}^{\prime} \mathrm{CL} \mathrm{L}^{\prime} 4(\mathrm{C}) \quad \mathrm{S}=45.5 \text { (standard entropy of cryst. } \mathrm{ThCl}_{4} \text { ) }
$$


A11 $\mathrm{H}$ and $\mathrm{G}$ relationships are in $\mathrm{kcal} / \mathrm{mol}$; a $11 \mathrm{~S}$ relationships in $\operatorname{cal} /(\operatorname{mol} \cdot \mathrm{K})$

All values refer to one mole of substance for the formula given.

\subsection{Conventions Regarding Pure Substances}

The values of the thermodynamic properties of the pure substances given in these tables are for the substances in their standard states. These standard states are defined as follows:

For a pure solid or liquid, the standard state at any temperature is the substance in the condensed phase under a pressure of one atmosphere.

For a gas the standard state at any temperature is the hypothetical ideal gas at unit fugacity, in which state the enthalpy is that of the real gas at the same temperature and at zero pressure.

The phase of a substance is indicated in parentheses at the end of the chemical formula. See Table A.

The values of $\Delta H f^{\circ}$ and $\Delta G f^{\circ}$ given in the tables represent the change in the appropriate thermodynamic quantity when one mole of the substance in its standard state is formed, isothermal1y at the indicated temperature, from the elements, each in its appropriate standard reference state. The standard reference state at $298 \mathrm{~K}$ for each element except phosphorus has been chosen to be the standard state that is thermodynamically stable at $298 \mathrm{~K}$ and at one atmosphere pressure. For phosphorus the standard reference state is the crystalline white form; the more stable forms have not been well characterized thermochemically. The same reference states have been maintained for the 
elements at $0 \mathrm{~K}$ except for the liquid elements bromine and mercury for which the reference states have been chosen as the stable crystalline forms. The standard reference states for the elements are indicated in the tables by the fact that the values of $\Delta \mathrm{Hf}^{\circ}$ and $\triangle \mathrm{Gf}^{\circ}$ are exactly zero.

The values of $\mathrm{S}^{\circ}$ represent the virtual or "thermal" entropy of the substance in the standard state at $298.15 \mathrm{~K}$, omitting contributions from nuclear spins and isotopic mixing. Where data have been available only for a particular isotope, they have been corrected when possible to the normal isotopic composition.

\subsection{Convention Regarding Solutions}

For all dissolved substances the composition of the solvent is indicated in parentheses following the chemical formula. Except in special cases, discussed below, the number of moles of the solvent associated with one mole of solute is stated explicitly. See Table A for the conventions used.

In some cases the concentration of the solute can not be specified. These are indicated as "AU" (aqueous, unspecified) for water solutions and by "U" for non-aqueous and mixed media. In all these cases the solution may be assumed to be "dilute".

The standard state for a non-dissociated solute in aqueous solution is taken as the hypothetical ideal solution of unit molality, which has been designated as "std. state, $m=1$ ". For strong electrolytes in aqueous solution the conventional standard state is the ideal 
solution of unit activity (unit mean molality). The designation "A" is used for strong electrolytes in the standard state and "AO" for undissociated species in water solution. In non-aqueous media two standard states are commonly used. For the mole fraction scale, "std. state, $x_{2}=1$ ", $x$ is added to the formula of the solvent. For the molal scale, "std. state, $\mathrm{m}=1$ ", either $\mathrm{s}$ or $\mathrm{M}$ is appended to the formula.

The value of $\Delta \mathrm{Hf}^{\circ}$ for a solute in its standard state is equal to the apparent molal enthalpy of formation of the substance in the infinitely dilute real solution, since the enthalpy of dilution of an ideal solution is zero. At this dilution the partial molal enthalpy is equal to the apparent molal quantity. At concentrations other than the standard state, the value of $\Delta H f^{\circ}$ represents the apparent enthalpy of the reaction of formation of the solution from the elements comprising the solute, each in its standard reference state, and the appropriate total number of moles of solvent. In this representation the value of $\Delta \mathrm{Hf}^{\circ}$ for the solvent is not required. The experimental value for a heat of dilution is obtained directly as the difference between the two values of $\Delta \mathrm{Hf}^{\circ}$ at the corresponding concentrations. At finite concentrations the partial molal enthalpy of formation differs from the apparent enthalpy.

The values of the thermodynamic properties tabulated for the individual ions in aqueous solution are based on the usual convention 
that the values of $\Delta \mathrm{Hf}^{\circ}, \Delta \mathrm{Gf}^{\circ}, \mathrm{S}^{\circ}$ and $\mathrm{CP}^{\circ}$ for $\mathrm{H}^{+}$(aq, std. state, $\mathrm{m}=1$ ) are zero. The properties of a neutra 1 electrolyte in aqueous solution in the standard state are equal to the algebraic sum of these values for the appropriate kinds and numbers of individual ions assumed to constitute the molecule of the given electrolyte. For an ionic aqueous species e.g., $\mathrm{HSO}_{4}^{-}$, the properties tabulated refer to that undissociated ion, i.e. they are not equal to the sum of those for its constituent ions. By adopting the above convention with respect to aqueous $\mathrm{H}^{+}$, it follows that the thermodynamic relation $\Delta G f^{\circ}=\Delta H f^{\circ}-T\left(\Delta S f^{\circ}+\mathrm{n} \cdot 0.5 S^{\circ}\left(H_{2}\right)\right)$ holds for individual ionic species, with $\mathrm{n}$ equal to the algebraic value of the charge. For neutral electrolytes the normal consistency relationship applies. See section 4 .

\section{Unit of Energy and Fundamental Constants}

Al1 of the energy values given in these tables are expressed in terms of the thermochemical calorie. This unit, defined as equal to 4.184 joules exactly, is generally accepted for the presentation of chemical thermodynamic data. Values reported in other units have been converted to calories by means of the conversion factors for molecular energy given in Table B.

Values in this report are consistent with the CODATA Fundamental Constants [73COD].

The formula weights in the tables have been calculated from the molecular formula using the 1969 Table of Atomic Weights [70IUP]. 
Values are given to four decimal places for convenience in the computer processing.

\section{Internal Consistency of the Tables}

The processes given in the reaction catalog have been obtained from the original articles, using consistent values for all subsidiary and auxiliary quantities. The original data were corrected where possible for differences in energy units, molecular weights, temperature scales, etc. Thus we have sought to maintain a uniform scale of energies for all processes in the tables and catalog. In addition the final tabulated values of the properties of a substance satisfy all the known physical and thermodynamic relationships among these properties. The quantities $\Delta H f^{\circ}, \Delta G f^{\circ}$, and $S^{\circ}$ at $298.15 \mathrm{~K}$ satisfy the relation:

$$
\Delta G f^{\circ}=\Delta H f^{\circ}-T \Delta S f^{\circ}
$$

to the precision given. The special case of solutions is discussed in section 2.4. Furthermore the calculated value of any thermodynamic quantity for a reaction is independent of the path chosen for the evaluation.

\subsection{Uncertainties}

The uncertainty reported for a selected value is "one sigma" as developed in the final least squares solution of the reaction data base. The magnitude of the uncertainty reflects the overall fit of the data in the entire network. It is not an estimate made by the data analyst based on how well various small sections of the network fit together. 
These uncertainties, when used in the usual "square root of sum of squares" formula overestimate the uncertainty of a process. This is because the selected values (answers) are highly correlated. Better estimates for processes that are in the data network are provided in the listing of the reaction catalog. These estimates allow for the correlations among values.

Since the selected values are computer generated, more places are given than may be warranted by the uncertainty. Although these extra figures in the absolute sense are not significant, the retention and use of these figures ensures that the experimental data from which these values are derived may be recovered with an accuracy equal to that of the origina 1 quantities. This is particularly important for enthalpies of solution, dilution and transition.

\subsection{Relationship to Other Tables of Thermodynamic Properties}

The chemical thermodynamic properties in the present table may be combined with those published by CODATA in order to calculate the change in a property for a process. However, we recommend against these values being combined with those in any other tabulation or with a property reported in an original research paper. In particular, we warn against indiscriminate combination with the NBS Technica1 Note 270 Series. A conversion to the NBS Technical Note 270 Scale will be made and included in that series.

There are several reasons for avoiding the combination of thermochemical data from more than one table. The most important is that different large-scale tables use different thermochemical properties 
of formation for substances that are ubiquitous in the rmochemical measurements. Outstanding examples are in the common inorganic acids and their ions. Another reason is that the groups preparing different tables may have relied on different measurements as the basis for selecting property values.

It is difficult to predict a priori how a change in one selected formation property would affect values assigned to other substances because of the way these are linked by complex networks. In general, it may be expected that the advantage of internal consistency of a table will be lost if values from several sources are combined and the experimental measurements may be reproduced poorly.

No general, simple algorithm can be suggested for overcoming this problem. If it becomes necessary to extend a table of data to substances other than those tabulated, the user is advised to consult the group that prepared the table about the procedure that he plans to use.

\section{Arrangement of the Tables}

The compounds in the tables are entered according to the Standard Order of Arrangement, (see Figure 1), by the principle of latest position. In this scheme, a compound is listed under the element occurring latest in the 1ist; water of hydration is neglected. Within a given element-table will be found all of the compounds of that element with elements occurring earlier in the order; the arrangement within a table follows the same ordering. 


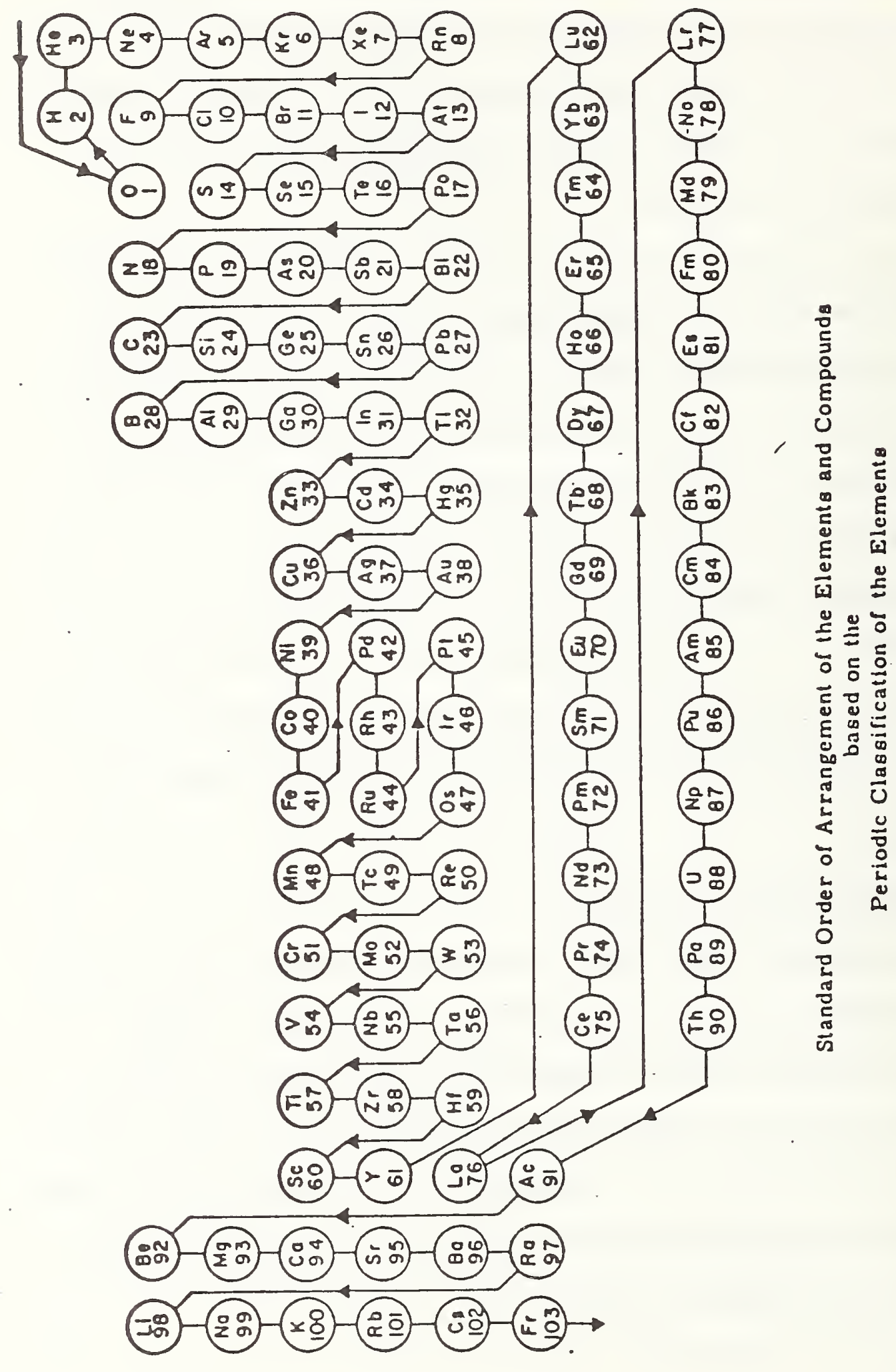

एँ 


\section{TABLE A: Physical State Conventions}

The following conventions are used to designate the physical state of a substance. These apply to the attached table and to the NBS Thermochemica 1 Data Bank. This information appears in a parenthetical expression appended to the molecular formula. Some of the explanations imply a thermochemical value, particularly those for solutions. These normally are used in describing enthalpy mea surements .

Basic Symbols

\section{Explanation}

Gaseous, e.g., HCL' (G) for HCl(g) Gaseous reference standard state for an element, e.g., $02(G S)$ for $\mathrm{O}_{2}(\mathrm{~g})$ Crystalline, e.g., NH4CL'(C) for $\mathrm{NH}_{4} \mathrm{Cl}(\mathrm{c})$ Crystalline reference standard state for an element, e.g., $\quad R B^{\prime}(C S)$ for $R b(c)$ Liquid, e.g., $\quad \mathrm{H} 2 \mathrm{O}(\mathrm{L})$ for $\mathrm{H}_{2} \mathrm{O}(l)$ Liquid reference standard state for an element, e.g., $\quad B R^{\prime} 2(\mathrm{LS})$ for $\mathrm{Br}_{2}(l)$

Amorphous

Glassy

Hypothetical standard state of the ideal aqueous solution at unit activity. For a neutral electrolyte the value of a property is equal to the algebraic sum of the values for the ions assumed to constitute the molecule of the electrolyte, e.g. HCL'(A) $=H+(A)+C L^{\prime}-(A)$. For an ionic species this notation is commonly used to refer to the undissociated ion as written. e.g. $\mathrm{HSO}_{4-(A)}$ 
Hypothetical standard state of the ideal aqueous solution at unit activity of the undissociated (non-ionized) species, e.g. $\mathrm{HF}(\mathrm{AO}), \mathrm{HF} 2-(\mathrm{AO})$. May also be used whenever the designation (A) could be ambiguous. Note that the descriptions $\mathrm{HSO}_{4}-(\mathrm{A})$ and $\mathrm{HSO}_{4}-(\mathrm{AO})$ are equivalent, but that $\mathrm{HF}(\mathrm{A})$ and $\mathrm{HF}(\mathrm{AO})$ are not. Aqueous solution of undefined, but usually dilute, concentration, e.g. XE'03(AU).

The symbols used above occasionally are modified by numbers to distinguish two substances in the same state that have the same molecular weight, as for isomers: (AU2), (C3). They are also used in combination with descriptive material, e.g. (C:HE'), (C:AL') etc. to mean "crystalline, hexagona1", "crysta1line, a1pha form" etc.

\section{Specia1 notations for substances in solutions}

The notations for the "state" of a substance in solution may combine a definition of the system, e.g. HC $\ell$ in 220 moles of water, and a specification of the thermochemical property associated with it. Usually the thermochemical property is the apparent integral enthalpy or free energy of formation or an absolute entropy, i.e. the formation properties of the solvent are not included. If a partial molal property is tabulated the notation D: (" $D$ " for "differential") occurs as the first term in the state bracket. The notations given below illustrate the differences for integral and differential (partial mola1) properties, and extrapolated values. Examples are given for aqueous, mixed, and non-aqueous solvents. 
HCL' (200H20)

$\mathrm{HCL}^{\prime}$ (D : 200H20) and $\mathrm{H} 2 \mathrm{O}\left(\mathrm{D}: \mathrm{HCL}^{\prime}+2 \mathrm{OOH} 2 \mathrm{O}\right)$

UCL ' 4 (HCL ' $04+50 \mathrm{H} 20)$

UCL ' 4 (HCL ' $\mathrm{O}_{4+}$ $50 \mathrm{H} 20: \mathrm{AU})$
An aqueous solution of specified composition, e.g. one mole of $\mathrm{HC} \ell$ in 200 moles $\mathrm{H}_{2} \mathrm{O}$. The value of $\triangle H f$ represents the apparent integral enthalpy of formation.

These represent the partial molal (enthalpy) of formation of the substance in a solution of specified concentration, e.g. the partial molal enthalpy of formation of $\mathrm{HC} l$ and $\mathrm{H}_{2} \mathrm{O}$ respectively, in a solution consisting of 1 mole $\mathrm{HC} l$ and 200 moles $\mathrm{H}_{2} \mathrm{O}$.

This describes a solute dissolved in a mixed solvent, e.g. one mole of $\mathrm{UCl}_{\ell_{4}}$ in a mixture of 1 mole of $\mathrm{HClO}_{4}$ and 50 moles $\mathrm{H}_{2} \mathrm{O}$. The value of $\Delta H f$ represents the apparent integral enthalpy of formation of the substance, $U C \ell_{4}$, in the medium. This represents a solute at an unspecified but usually dilute concentration in a solvent mixture of fixed composition. 


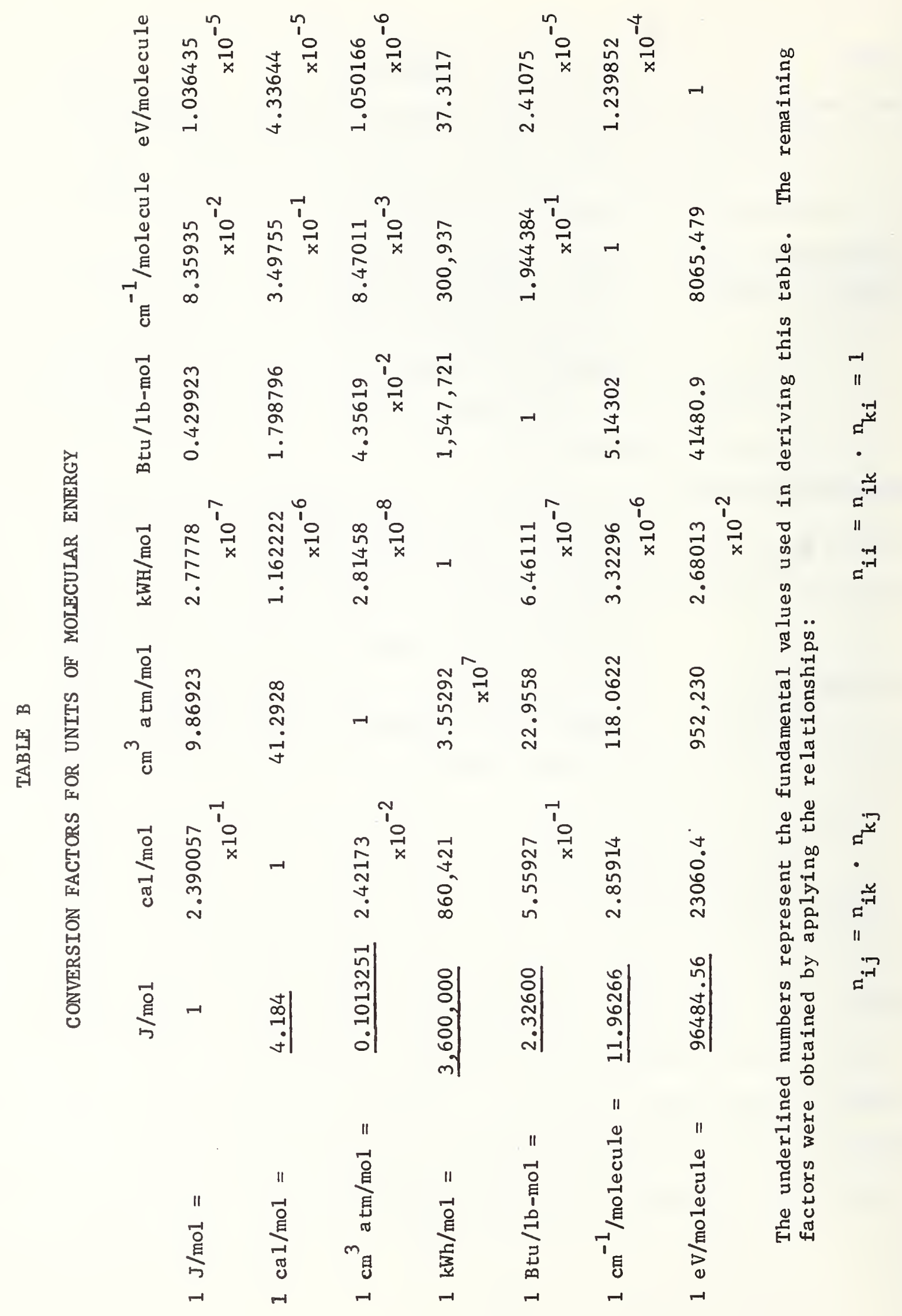


II. Tables of Values for $\Delta H f^{\circ}, \triangle G f^{\circ}$ and $S^{\circ}$ at $298.15 \mathrm{~K}$ for the Compounds of Thorium and the Reaction Catalog 
告

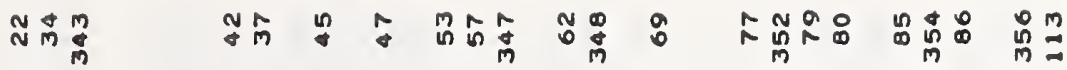

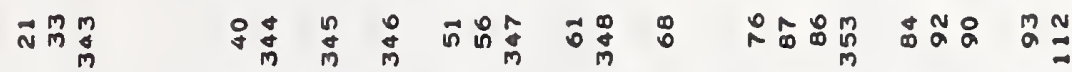

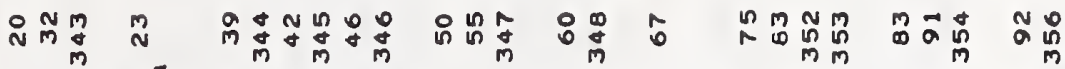

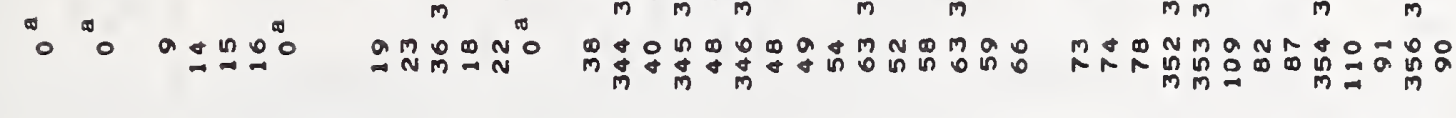

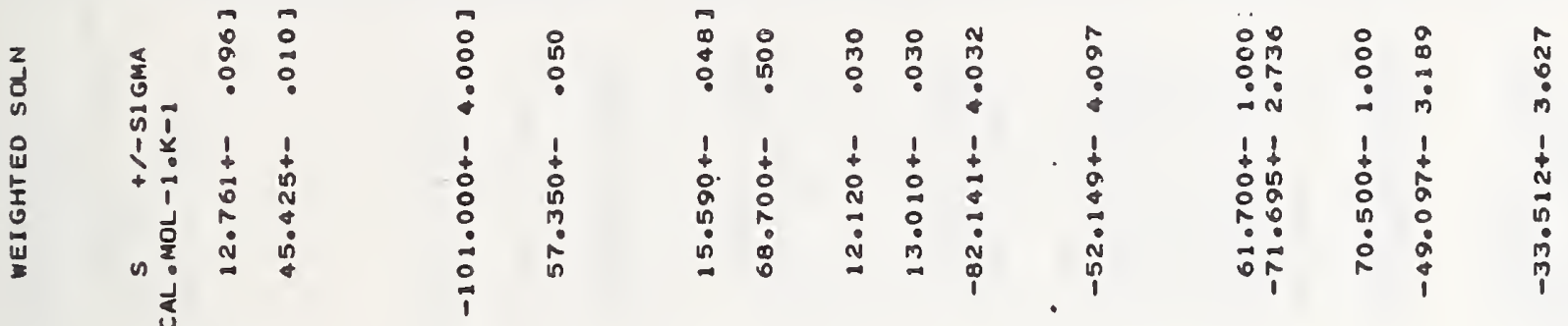

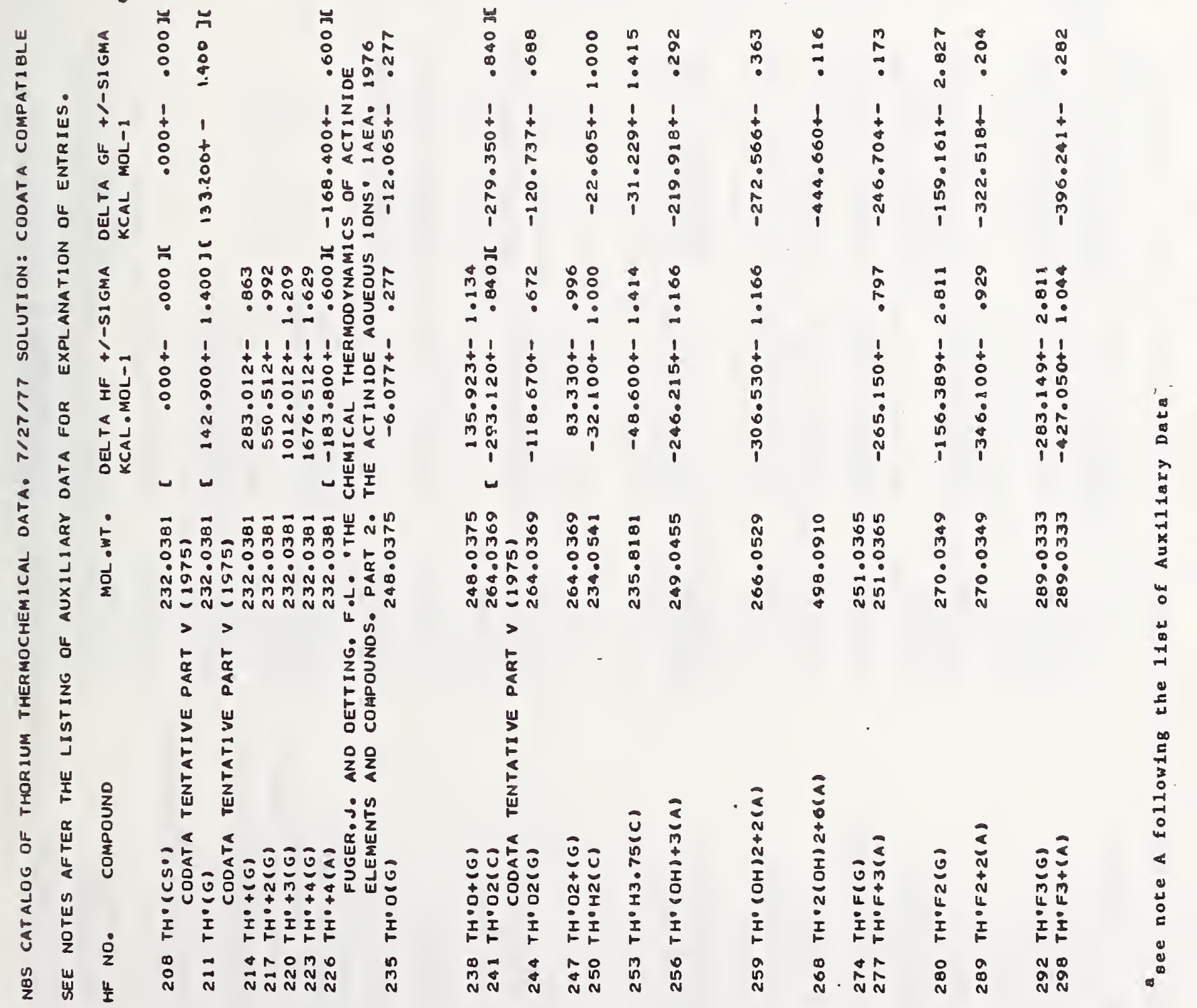




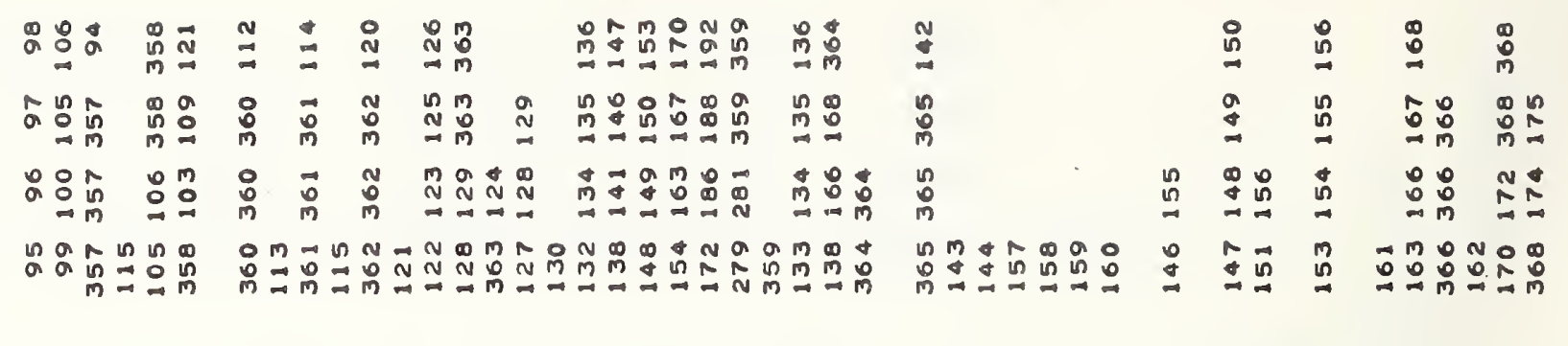

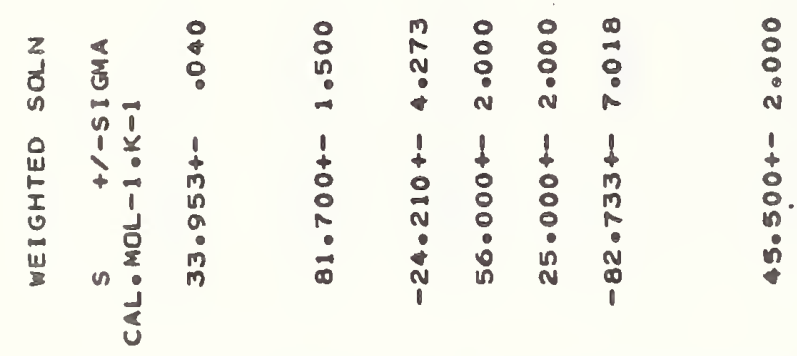

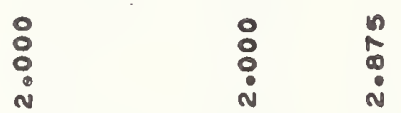

$\begin{array}{ll}a & : \\ i & \vdots\end{array}$

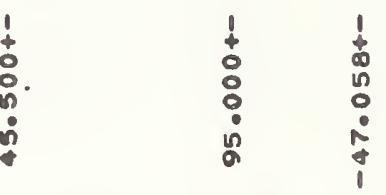

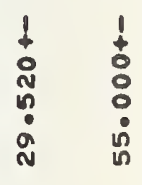

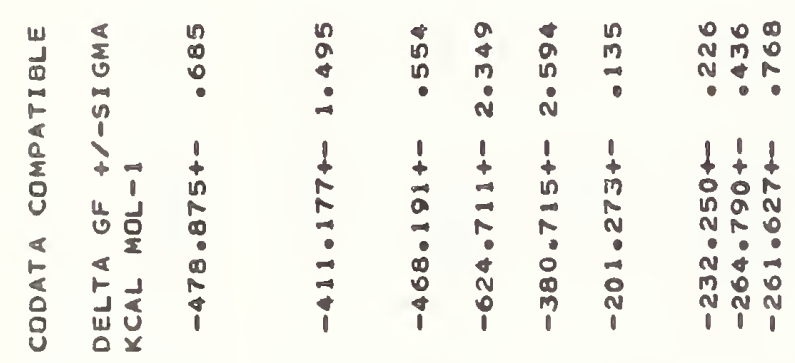

$\begin{array}{lll}\infty & 0 \\ 0 & 0 & \vdots \\ 0 & 0\end{array}$

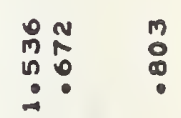

$\begin{array}{lll}1 & 1 & 1 \\ \vdots & + & 0 \\ 0 & 0 & 0 \\ 0 & 0 & 0 \\ 0 & 0 & 0 \\ 0 & 0 & 0 \\ N & 0 & 0 \\ N & N & N\end{array}$

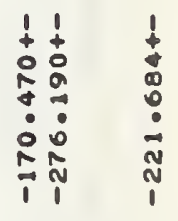

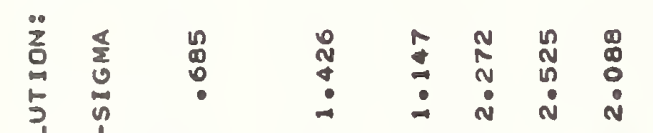

+

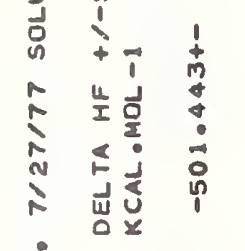

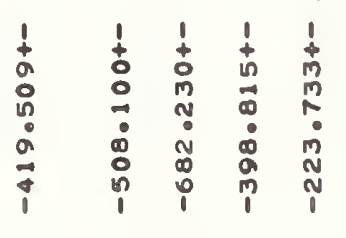

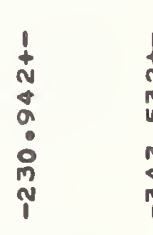

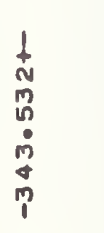

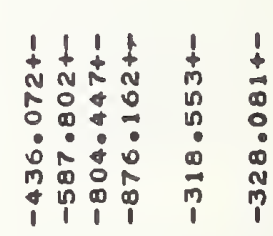

음

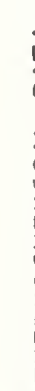

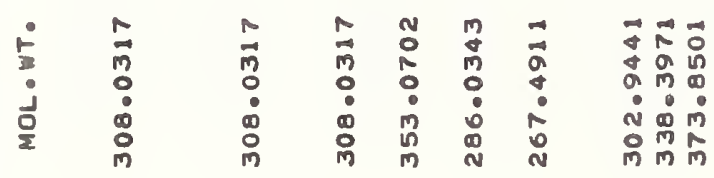

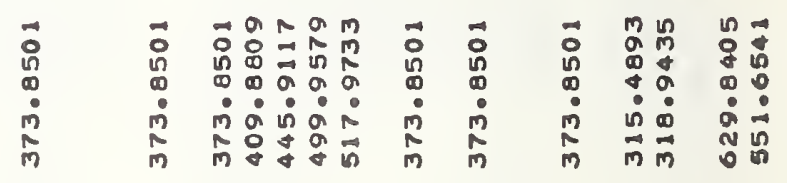

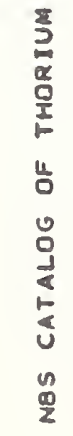

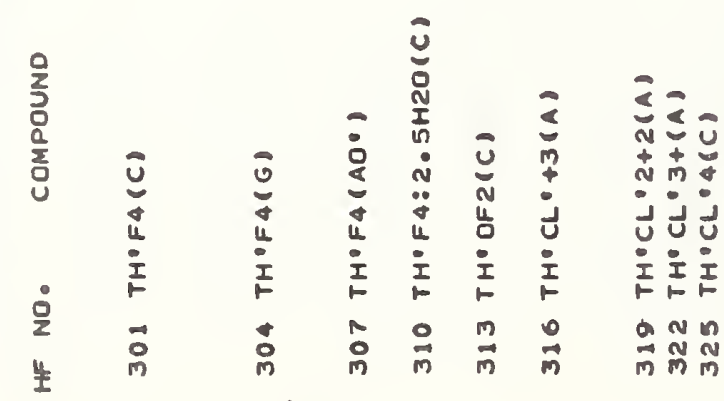

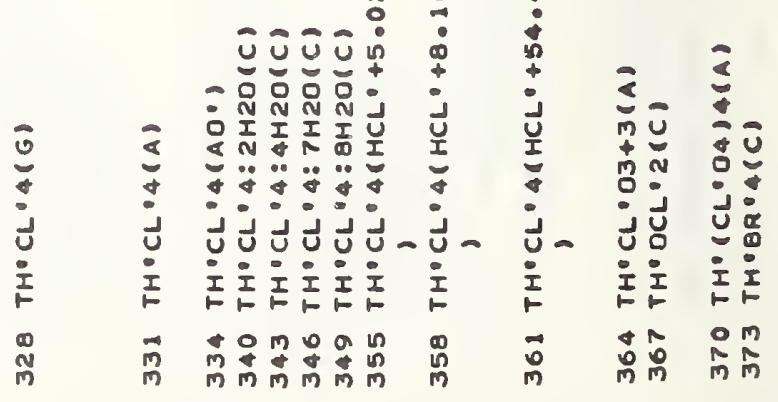

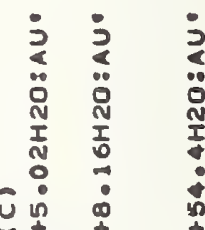




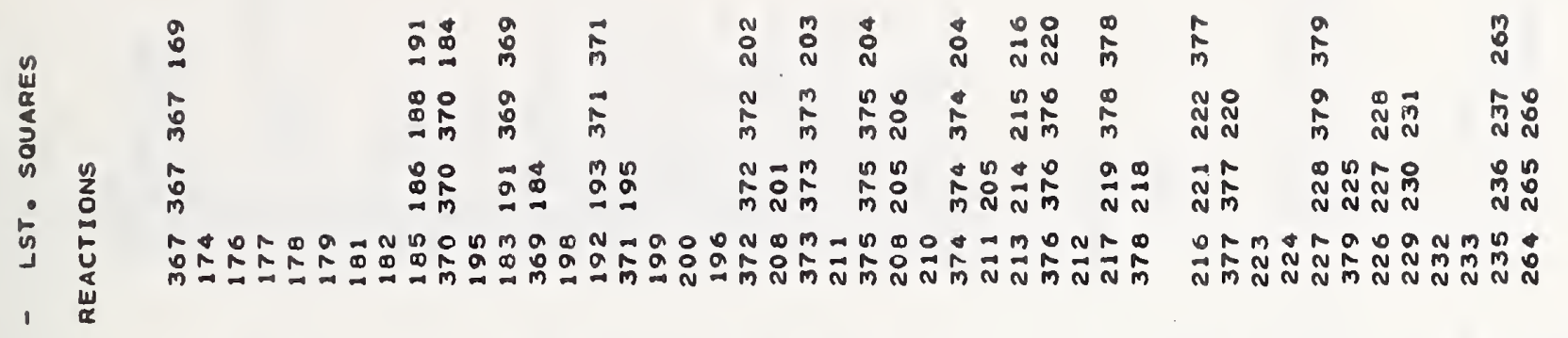

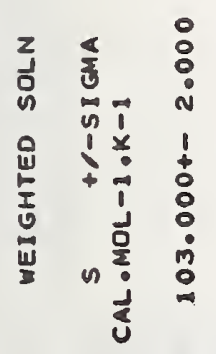

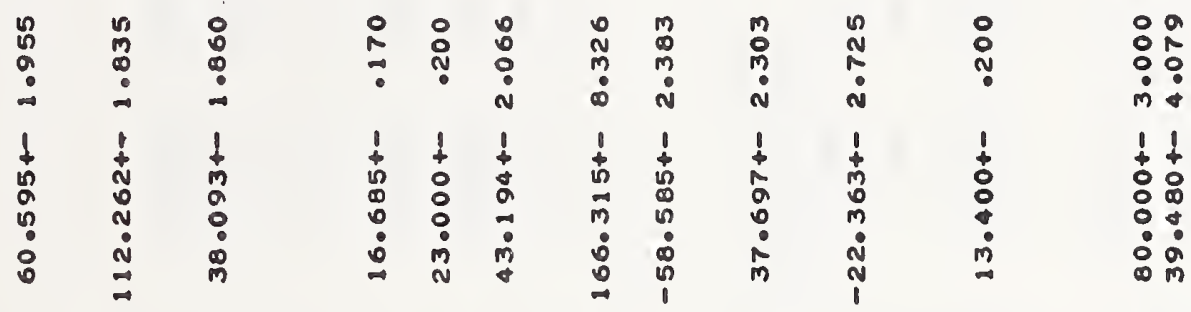

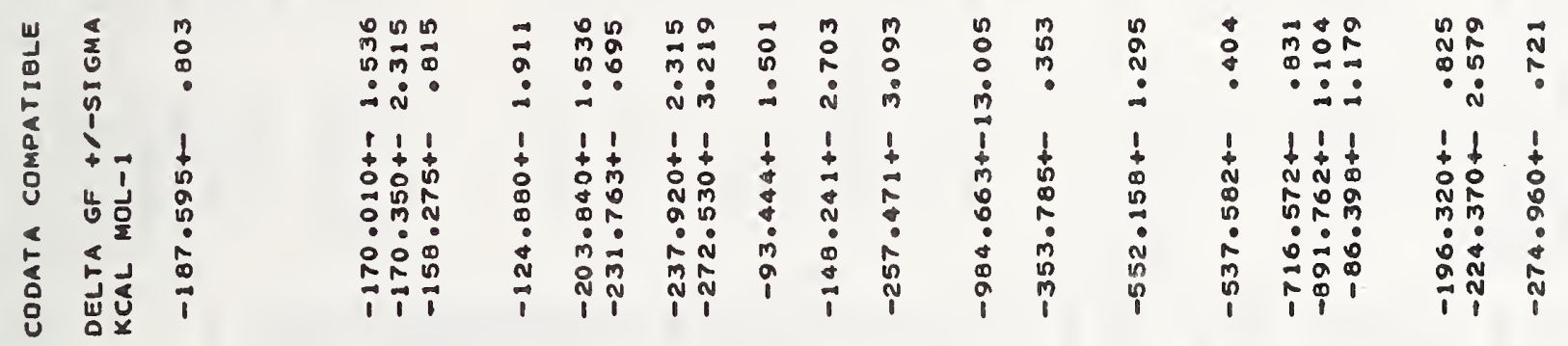

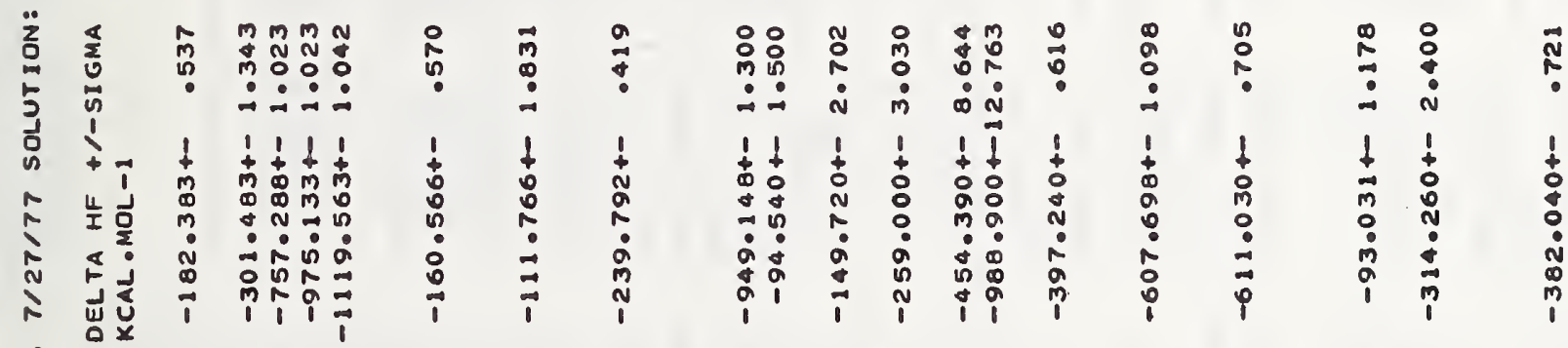

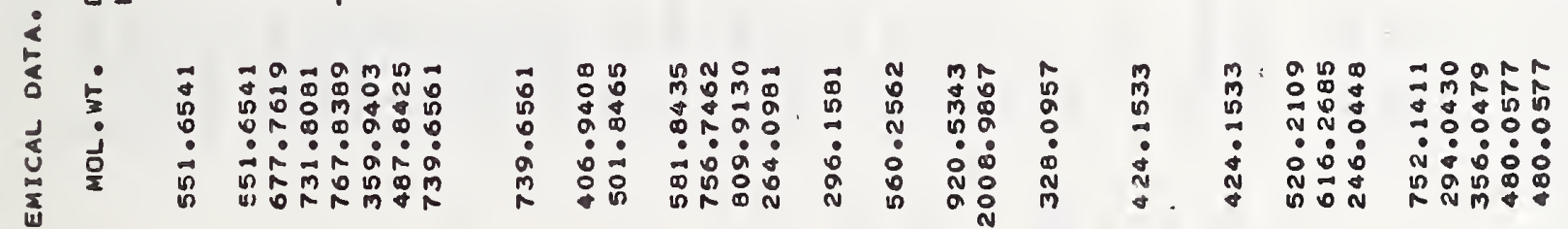

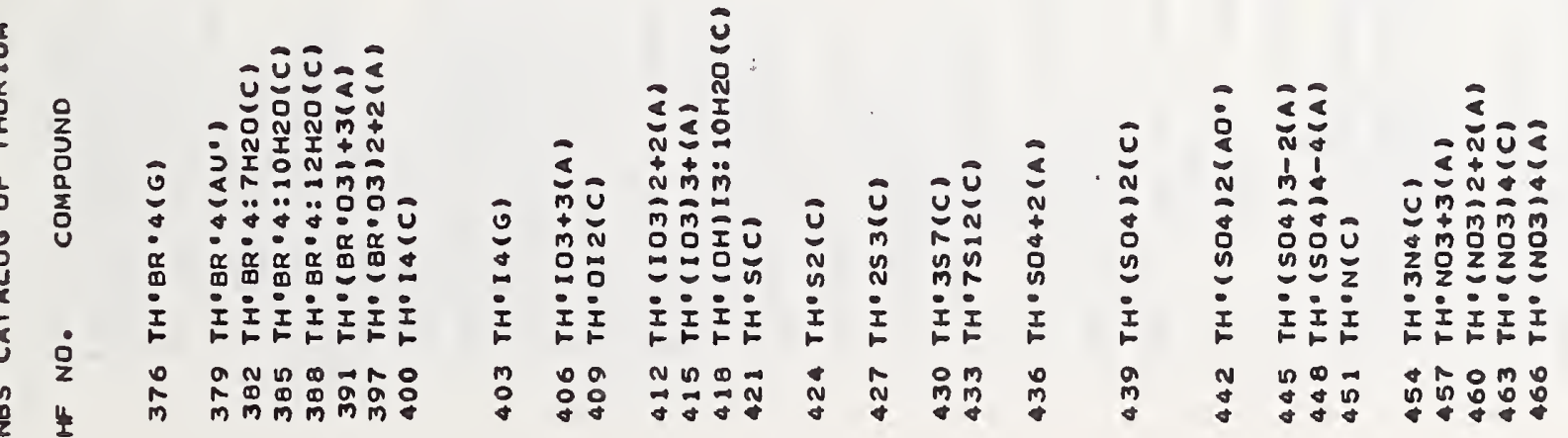




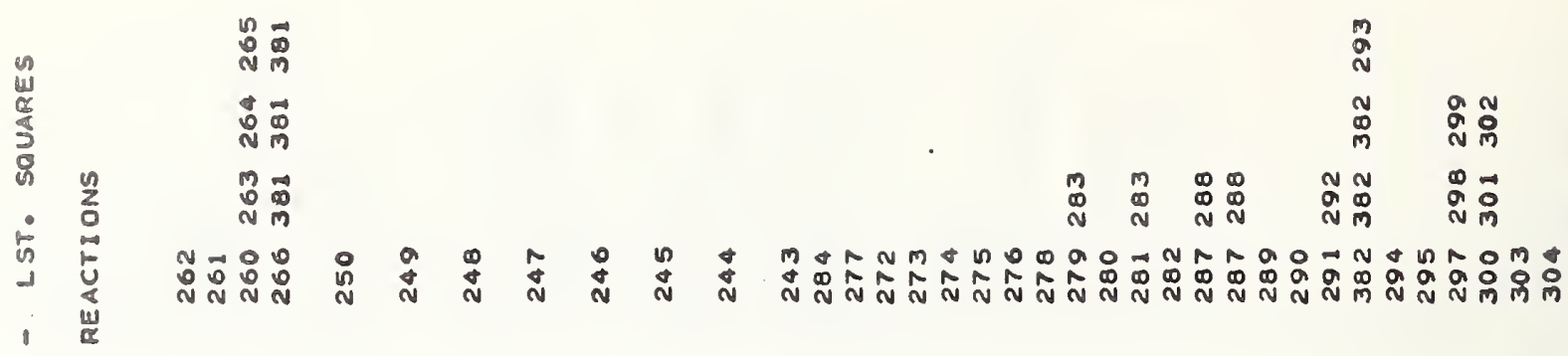

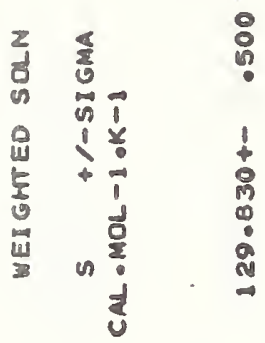

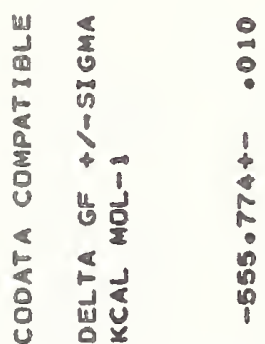

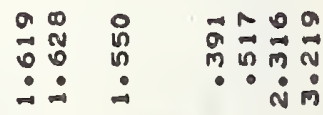

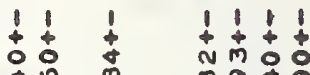

薄

$\therefore \dot{0} \quad \dot{0}: \dot{0}$

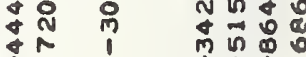

(1)

矛

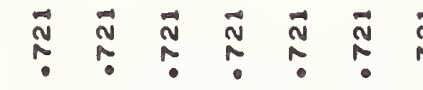

(2)

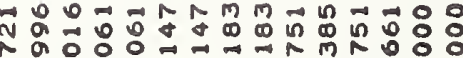

11

111

+

$1+5$

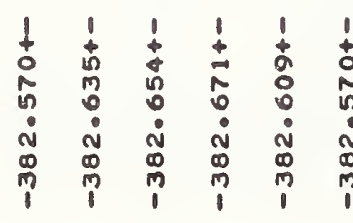

$\dot{-1} \dot{-} \dot{-} \dot{-} \dot{-} \dot{-} \cdot$

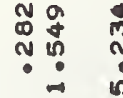

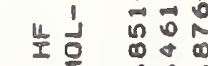

$\stackrel{\circ}{\circ}$

$\ll$

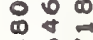

N 岂远

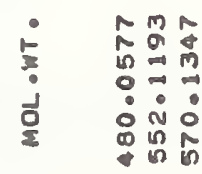

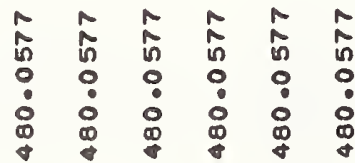

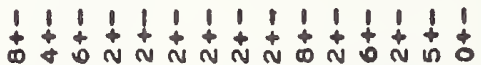

N N

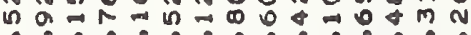

$\dot{0} \dot{0} \dot{0} \dot{0} \dot{0} \dot{0} \dot{0} \dot{0} \dot{0} \dot{m}$

N $=0007$

m,

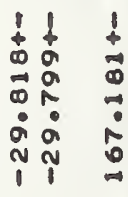

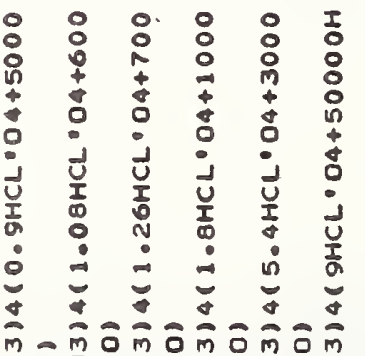

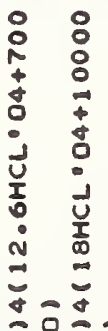

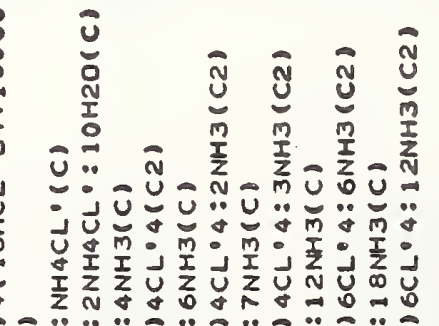

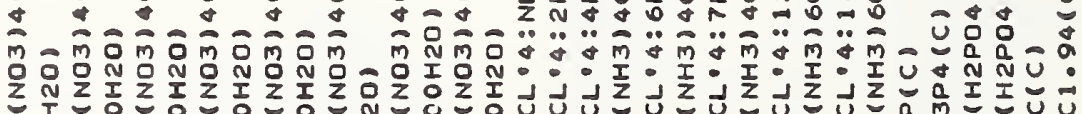

I

I논

$i_{i}^{i} i_{i}^{i}$

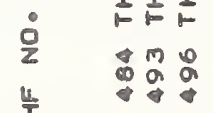

\%

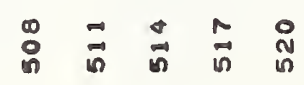

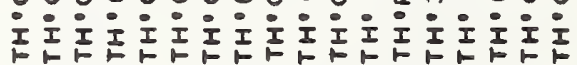

은

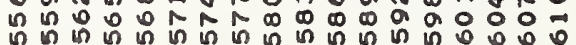

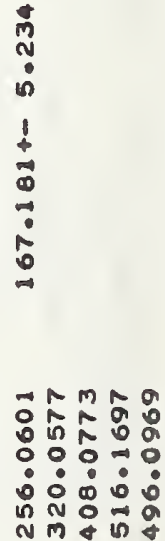




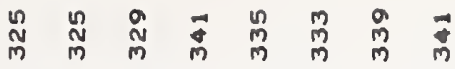

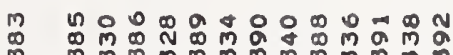

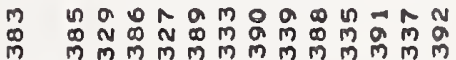

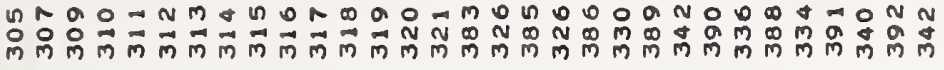

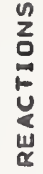

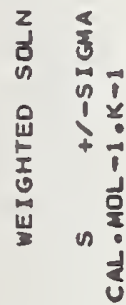

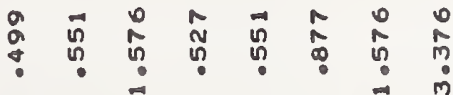

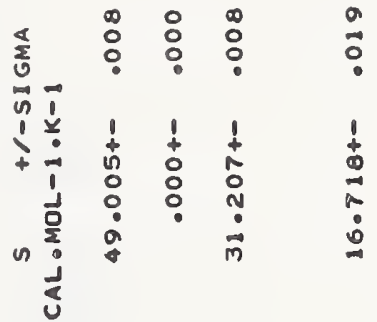

$\stackrel{\circ}{0}$

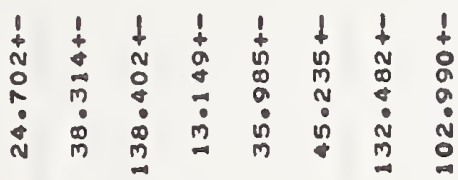

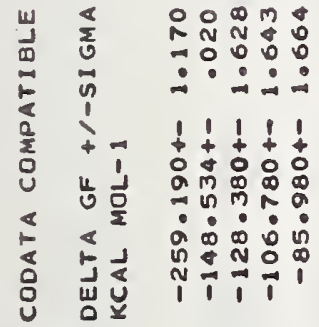

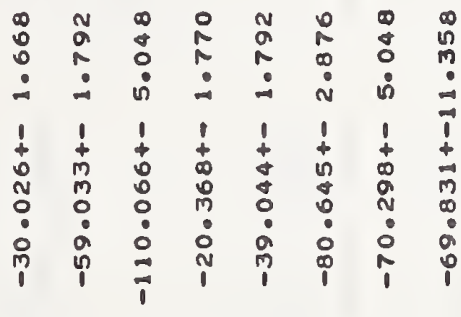

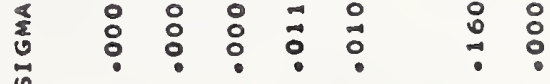

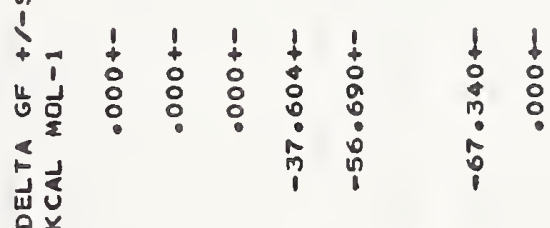

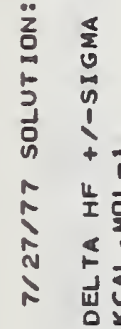

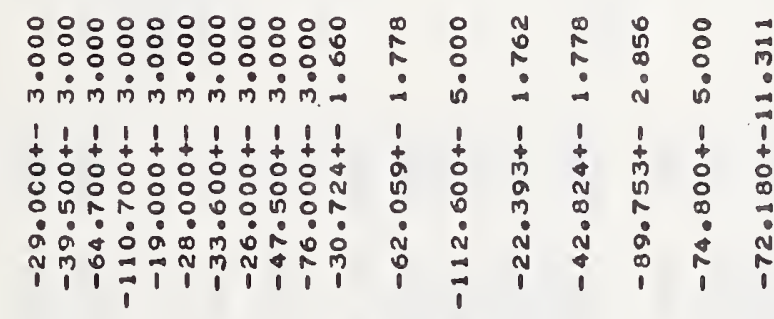

인

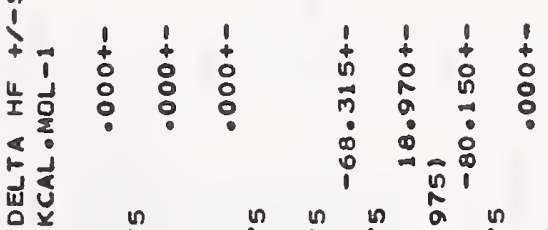

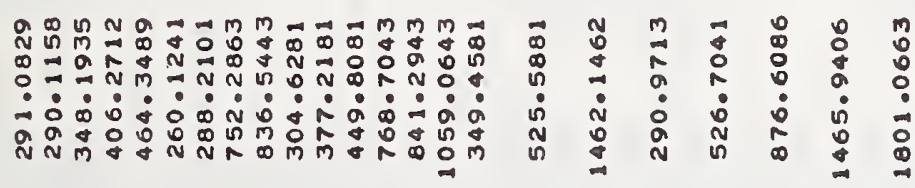

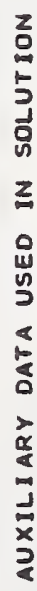

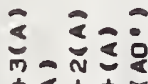

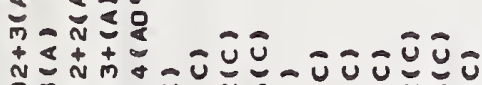

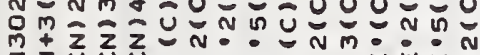

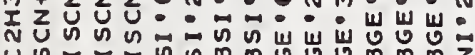

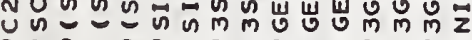

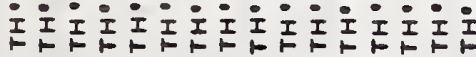

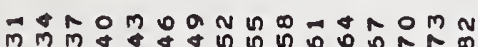

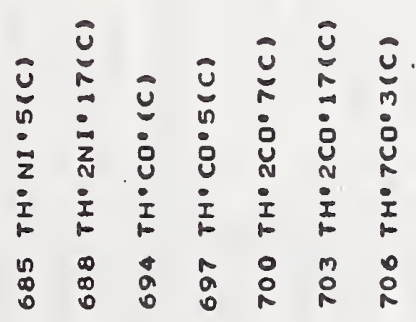
.

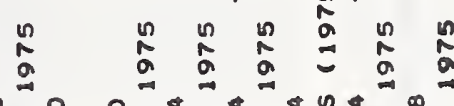

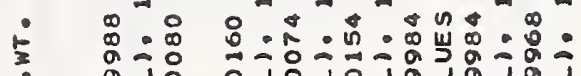
ग $\quad$ oj

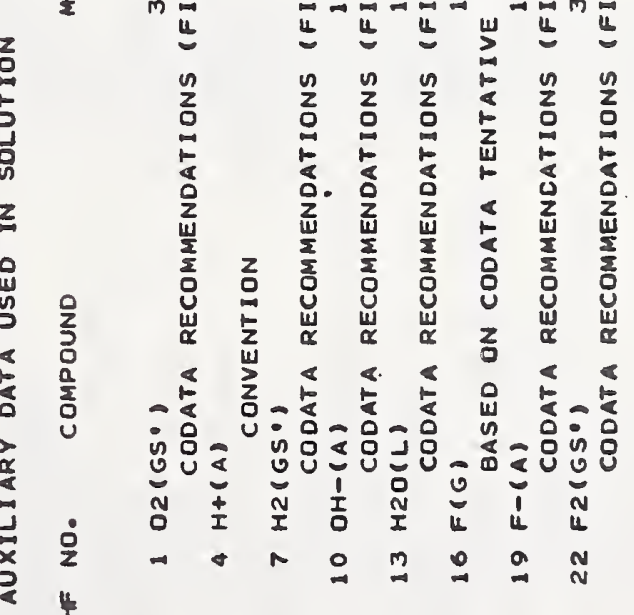




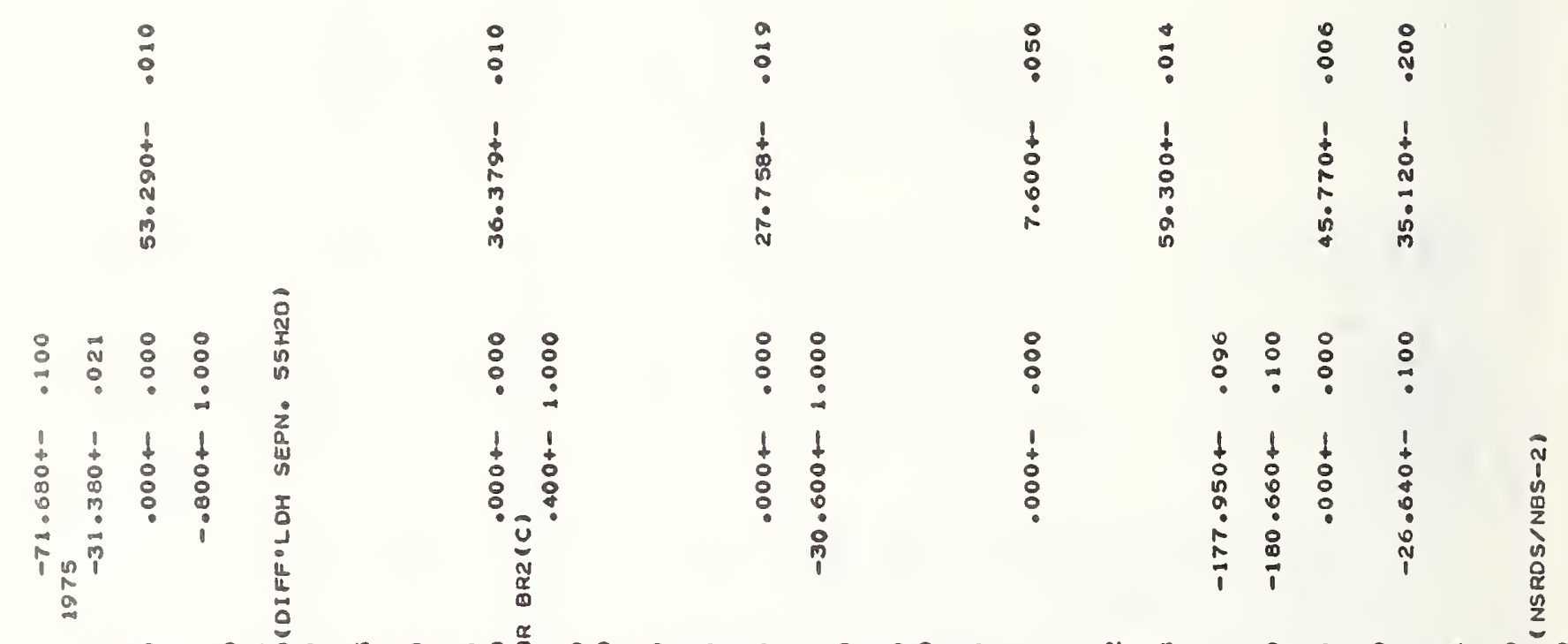

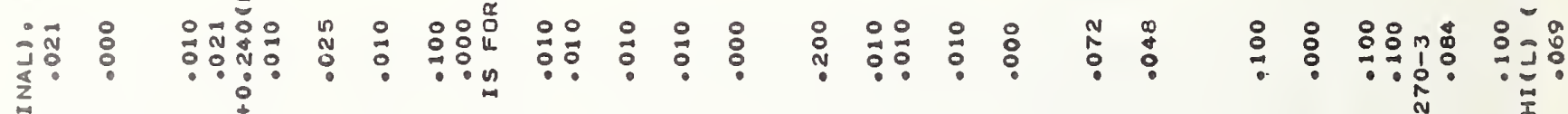

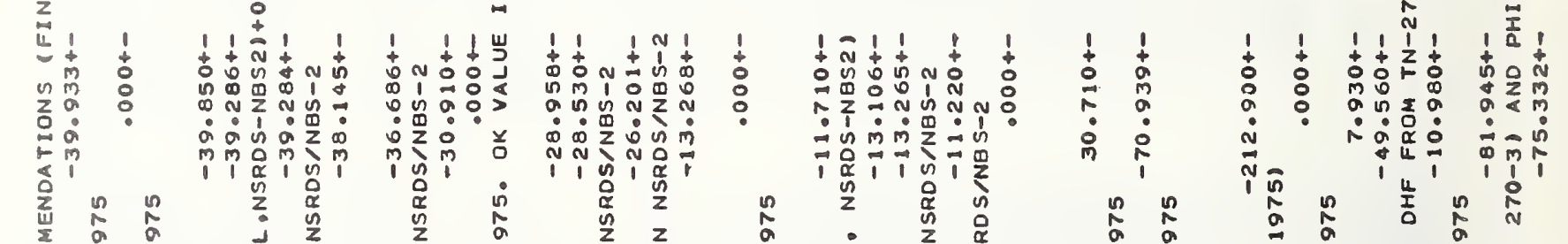
紊等

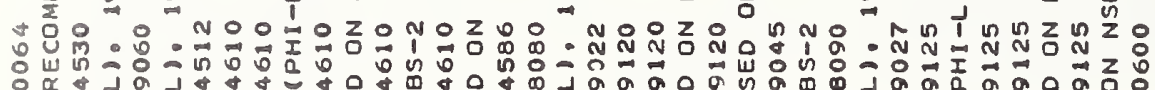

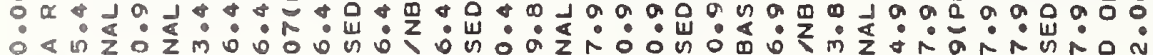

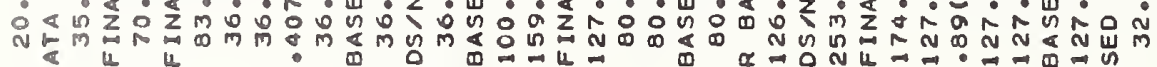

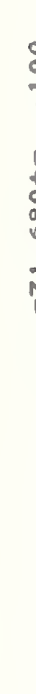

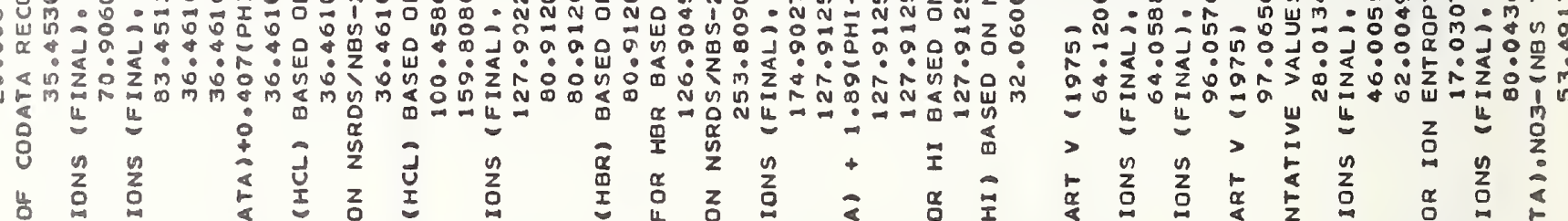

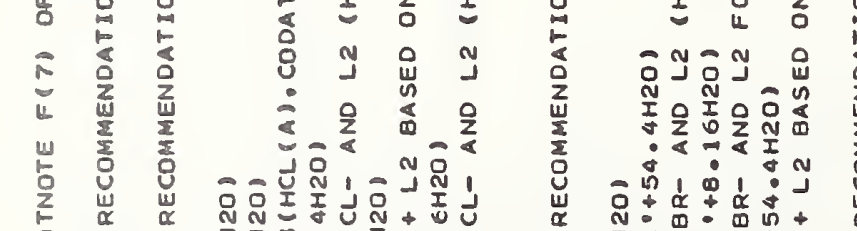

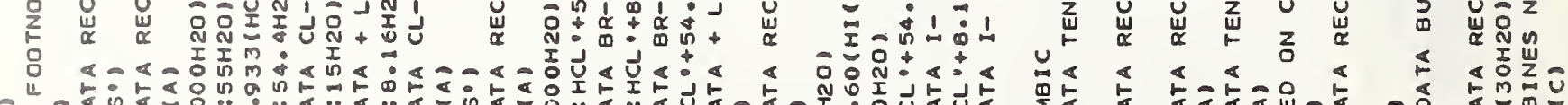

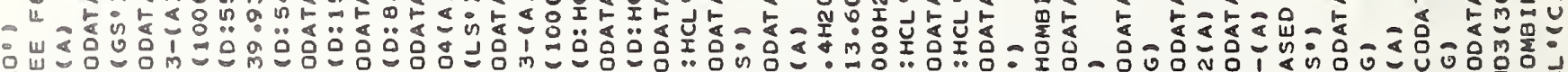

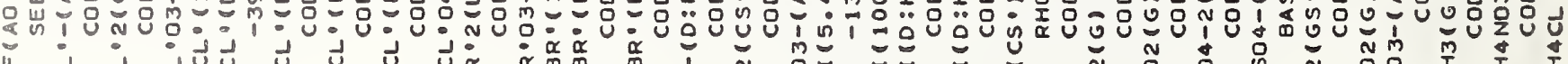

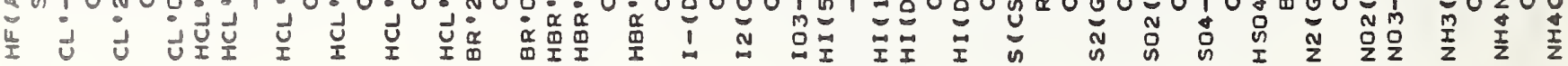

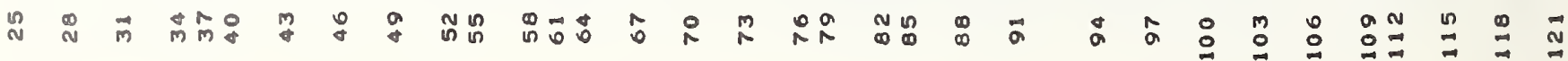




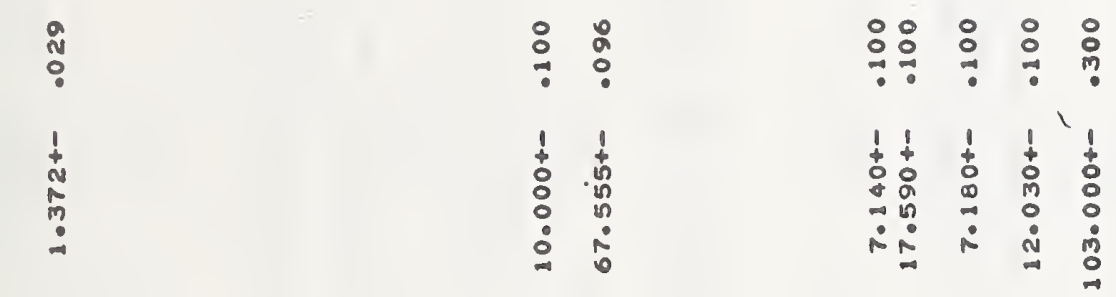

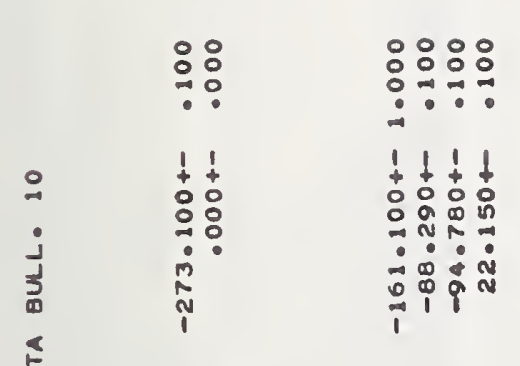

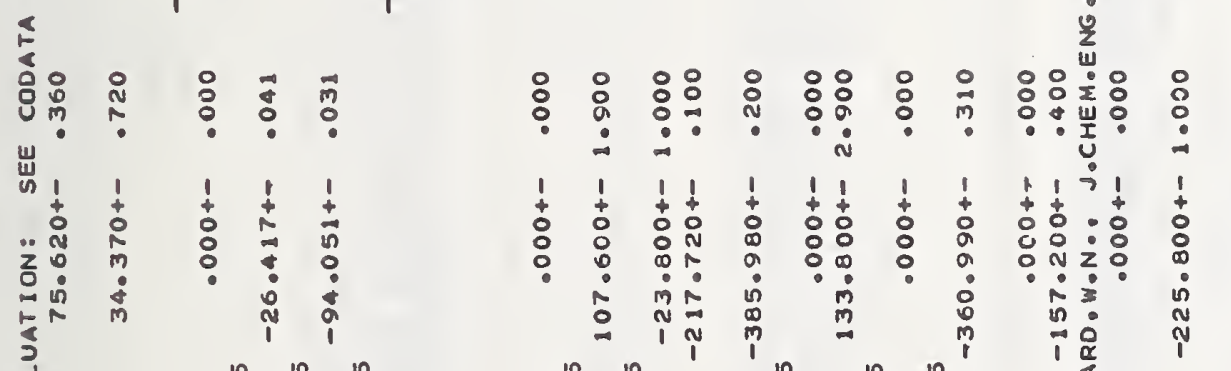

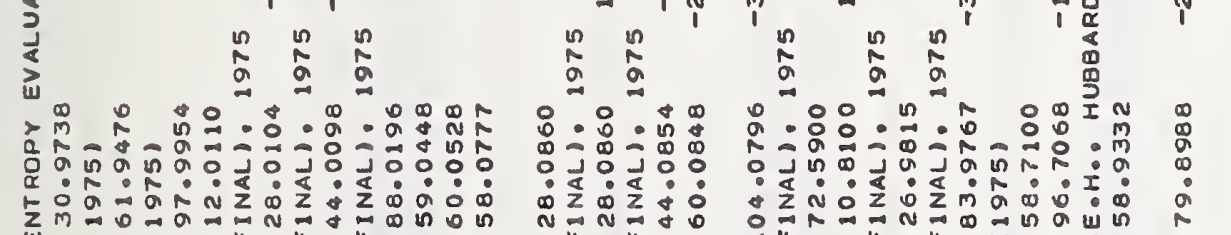

$\sum_{u} m=0$

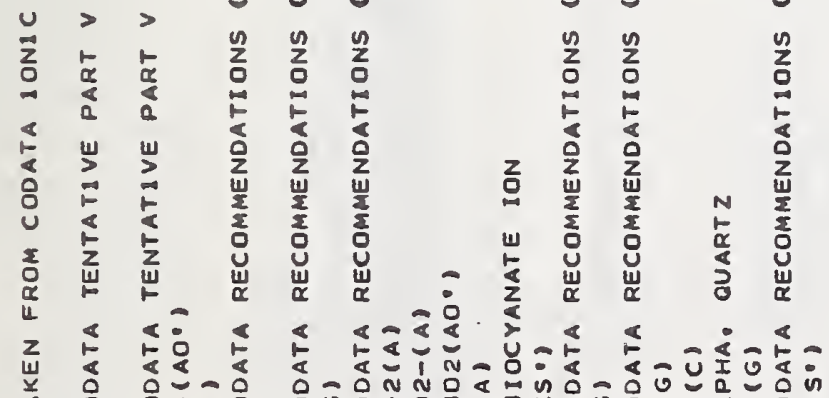

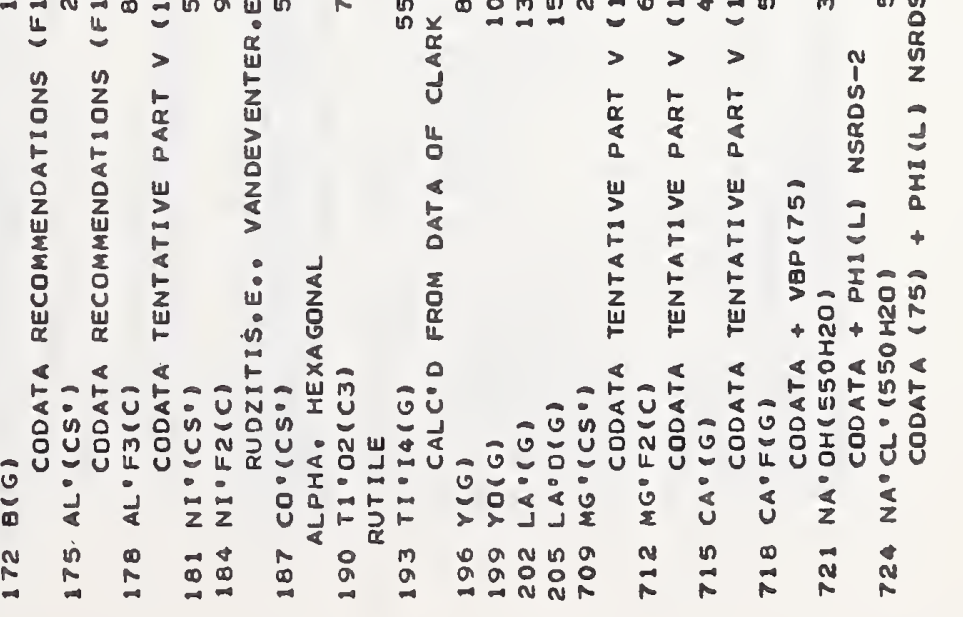




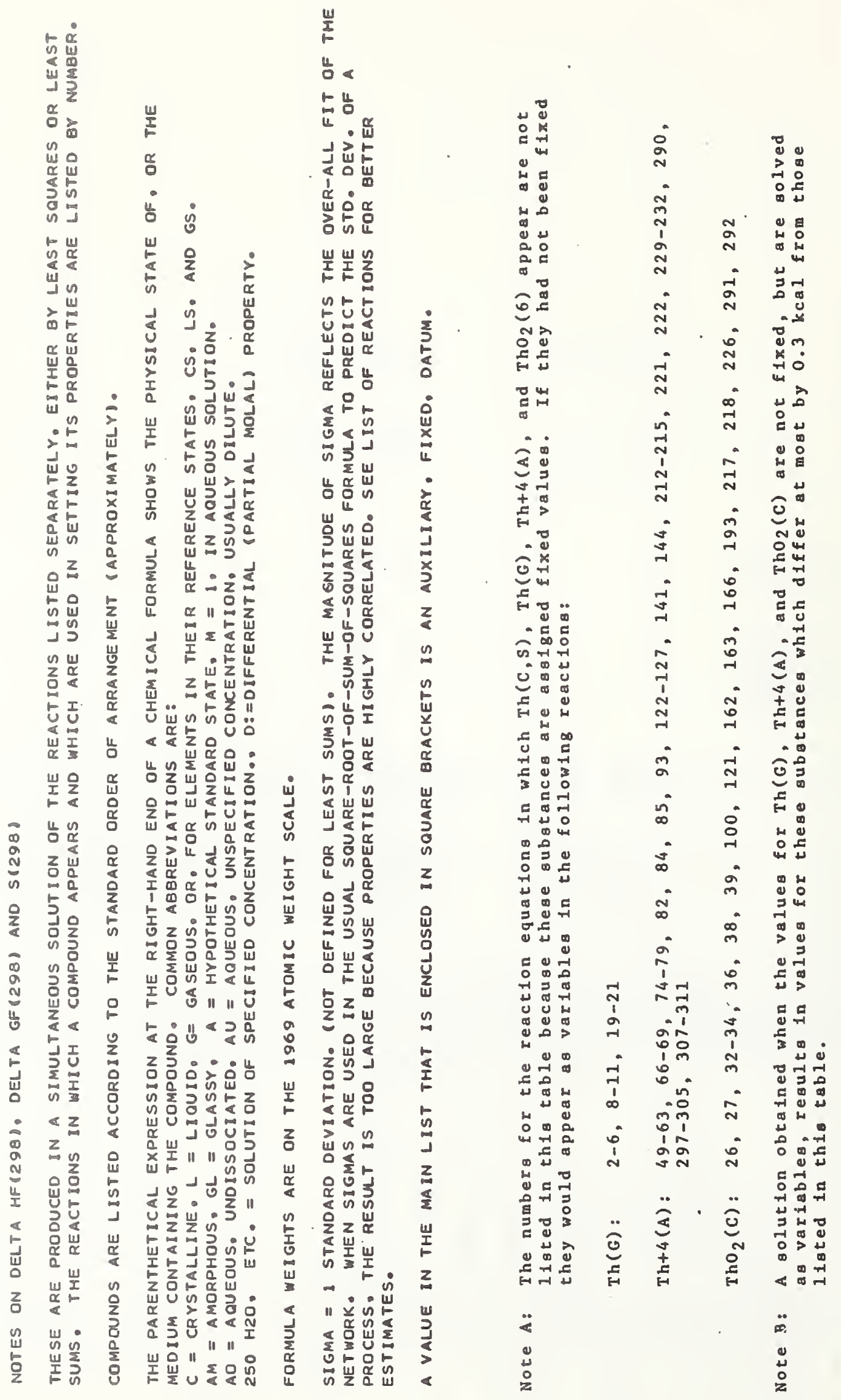


岁总总

娈兽

新

竞

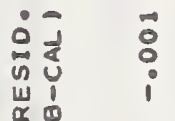

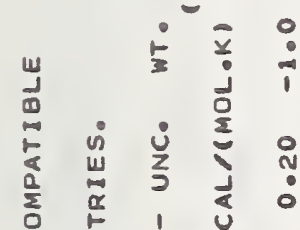

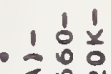

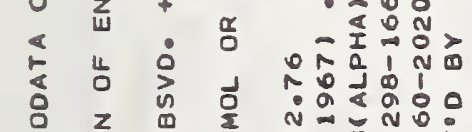

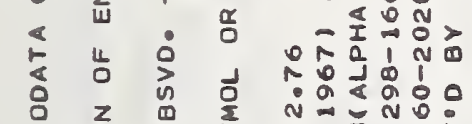

z 0 更

要: 0

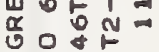

ษ两思

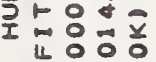

西品: :

일

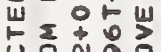

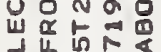

岃以

红思的

《०: "

-

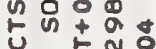

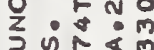

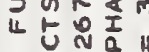

: $\frac{2}{2}: \frac{1}{4}$

空" 11 主并

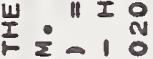

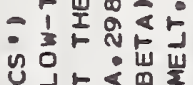

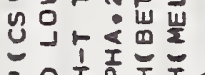

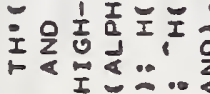

n $I \ddot{x}=0$ z

造

-

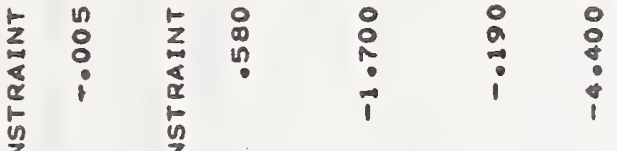

$\because \quad$ J

วั

u

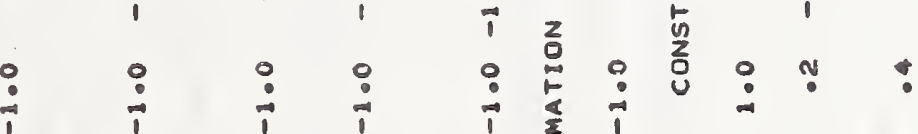

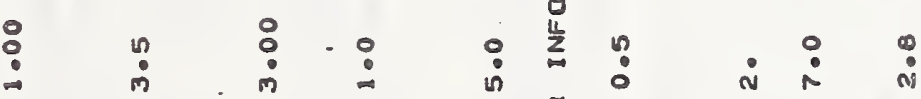

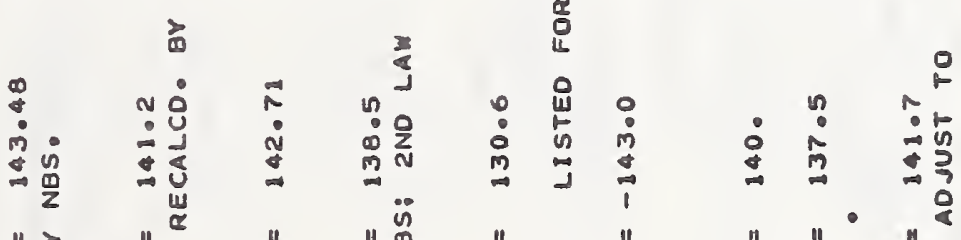

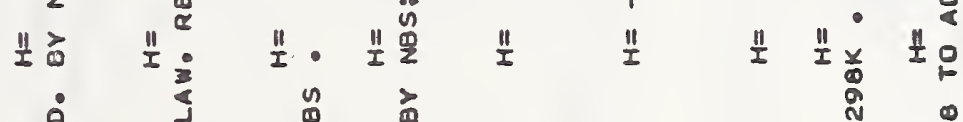

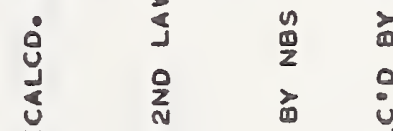

น

$\frac{1}{10}$

渵

2.

前前立

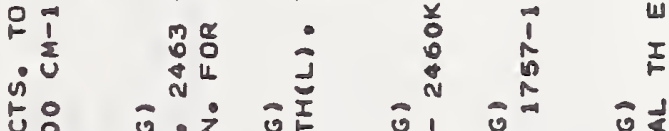

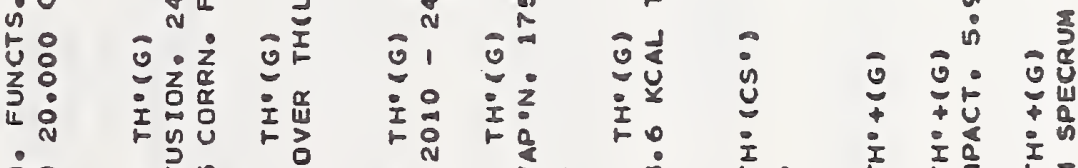

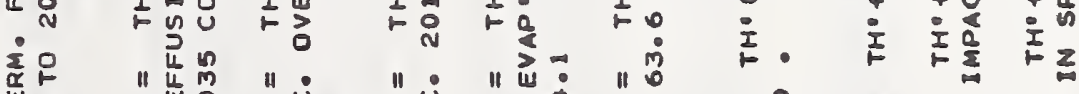

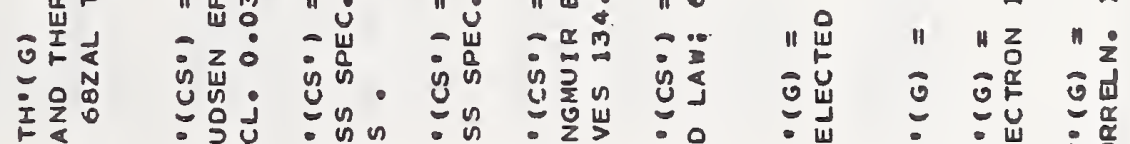

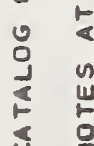

崖 


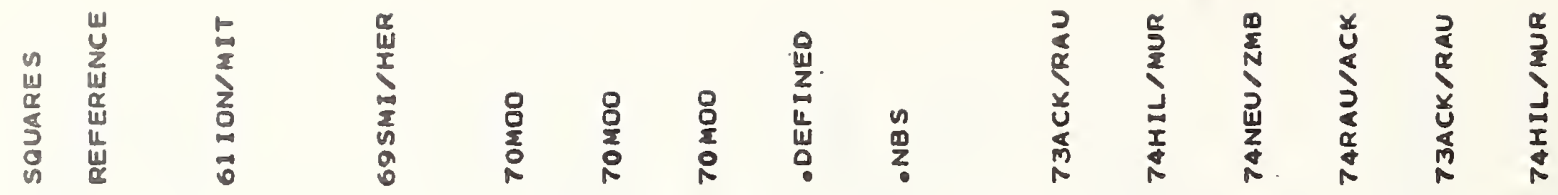

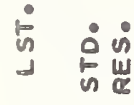

竞

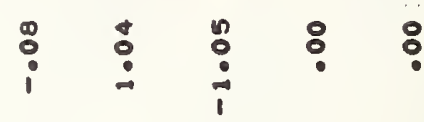

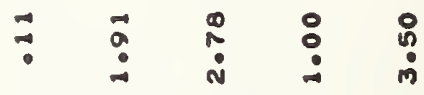

竎

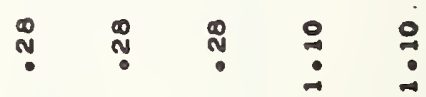

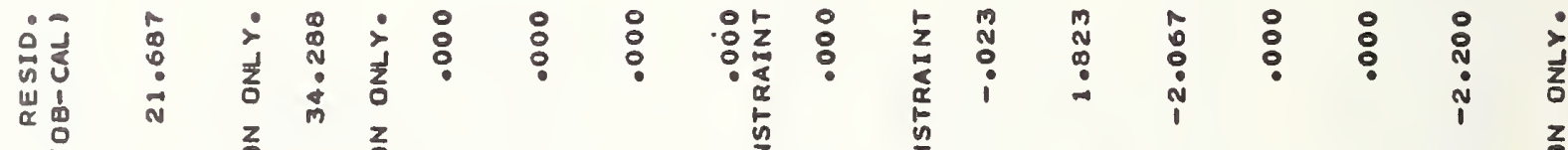

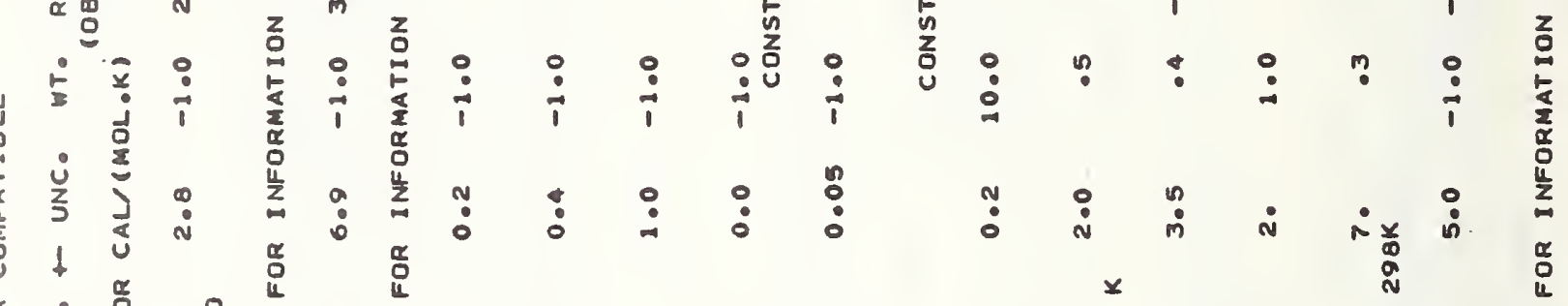

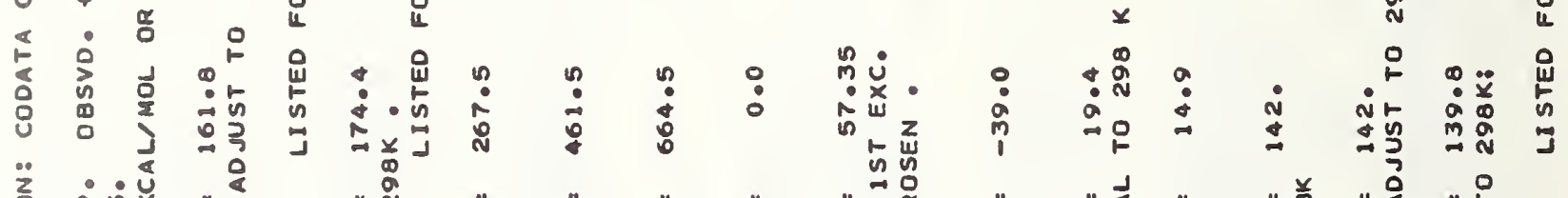

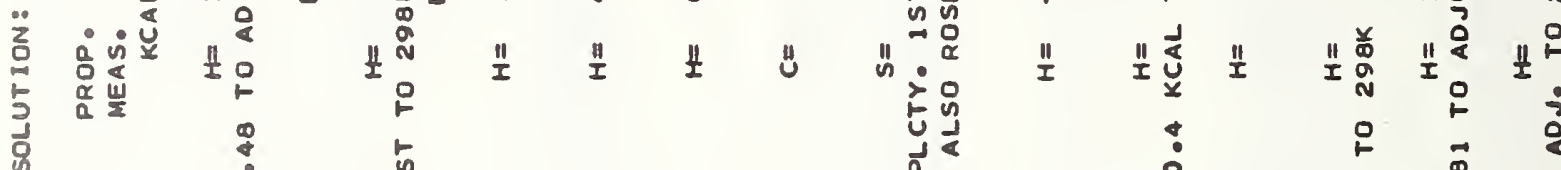
$\begin{array}{llllll} & & \\ 0\end{array}$

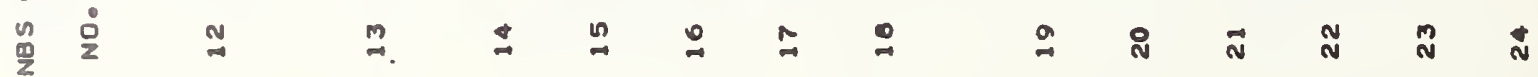




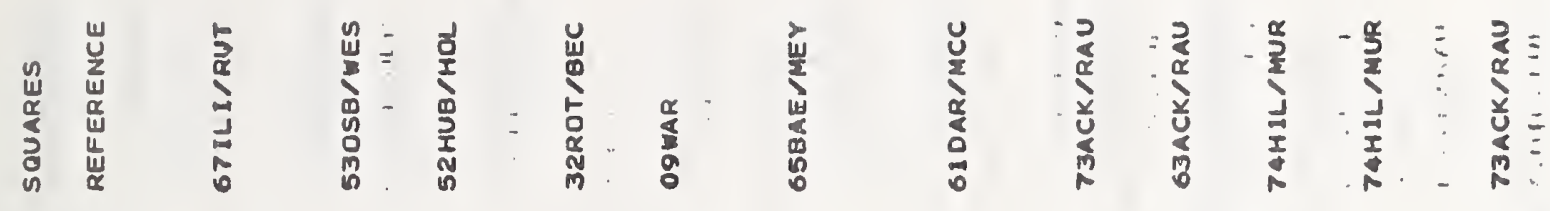

高总兽

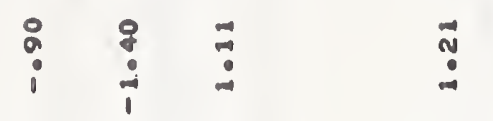

新

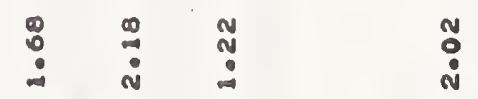

竎离

ำ

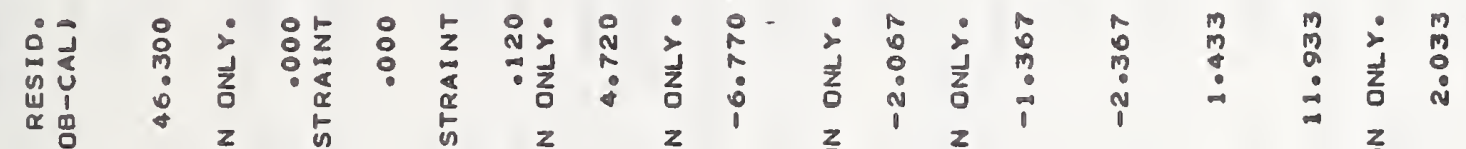

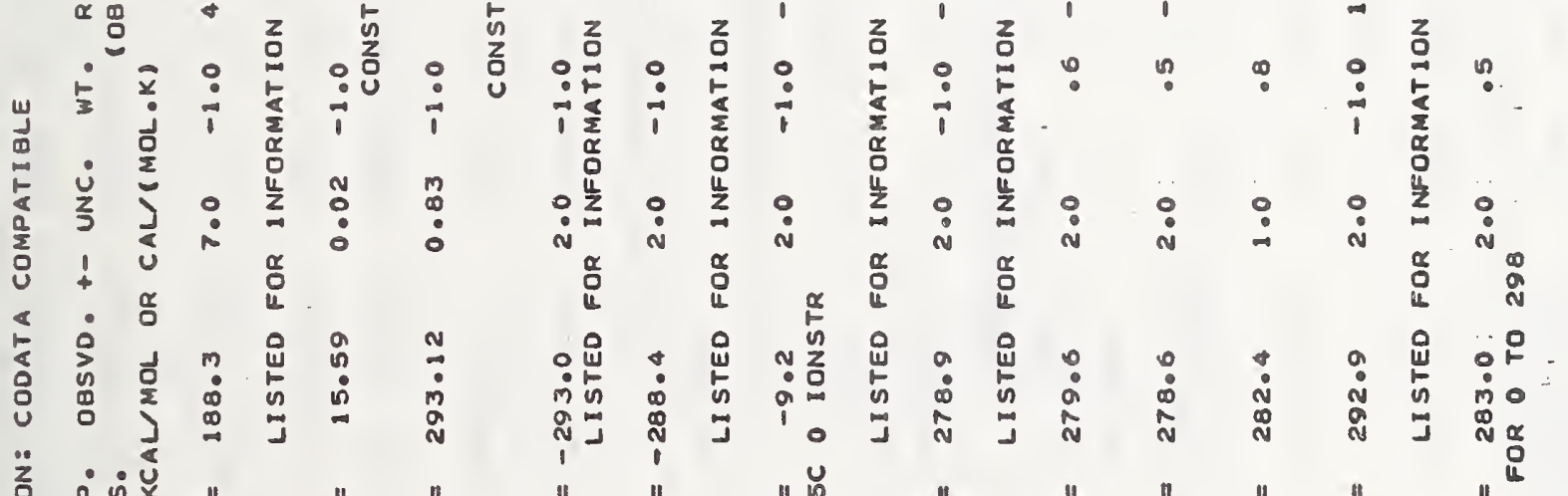
突

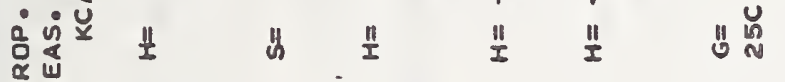
8

일

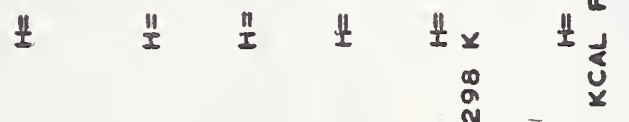

ह

辛

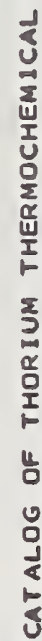

$\div:$

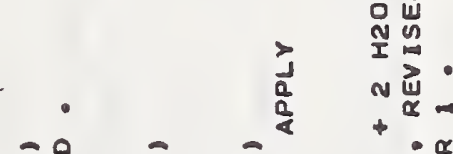

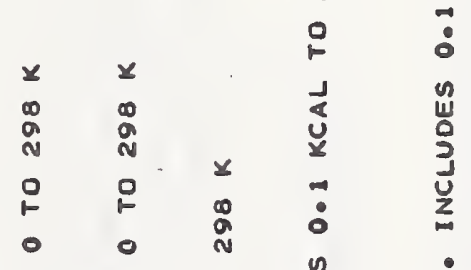

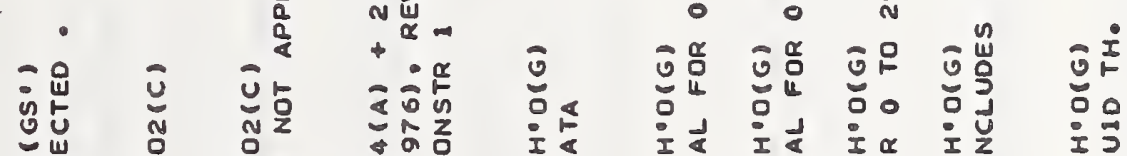

弅出

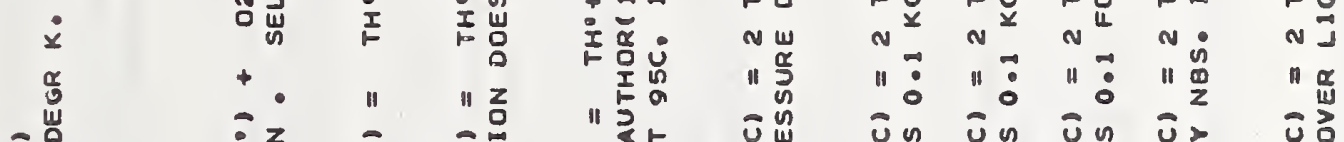

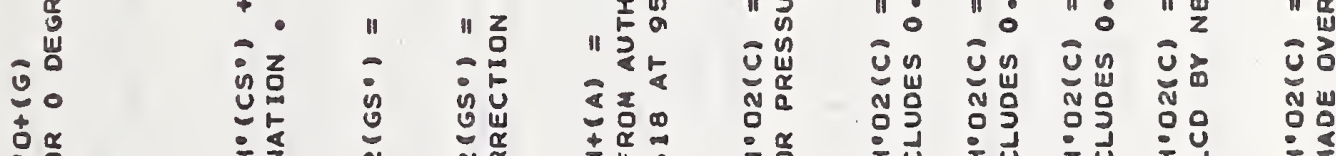

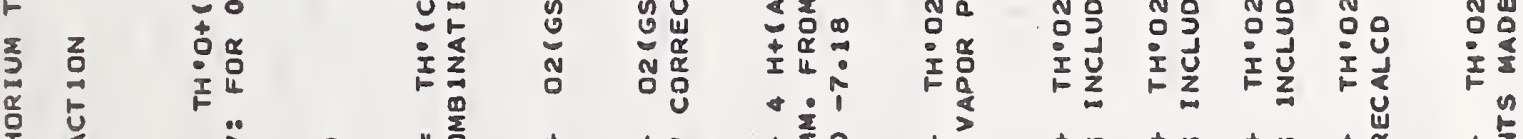

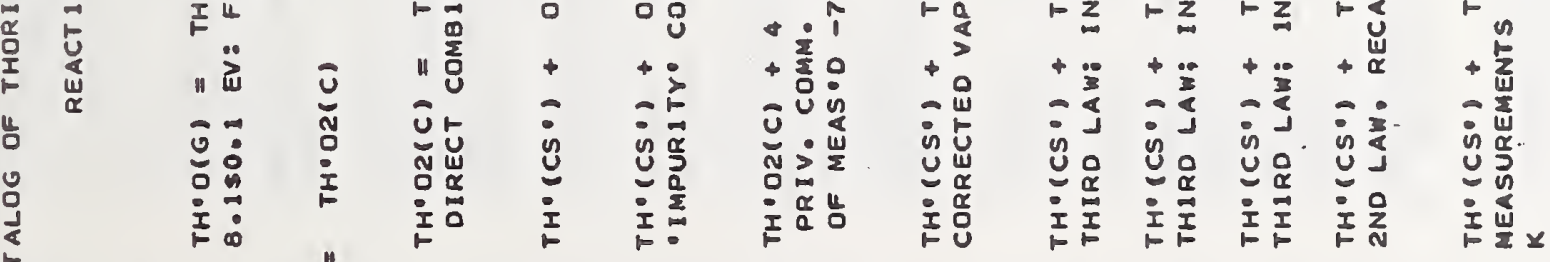




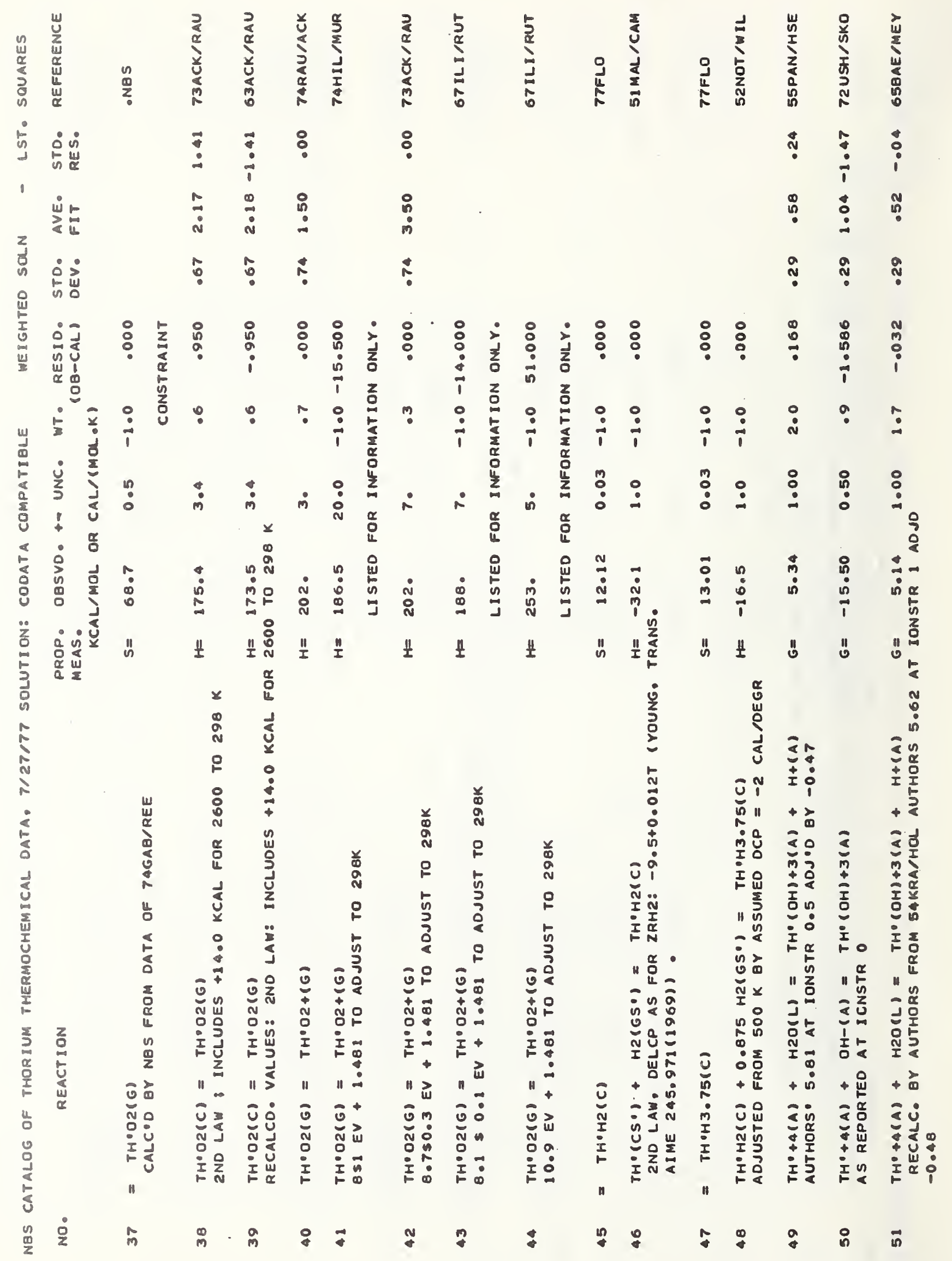




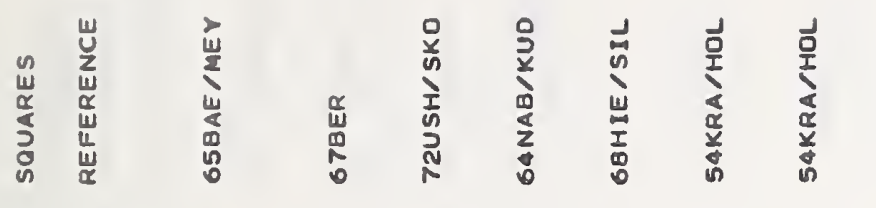

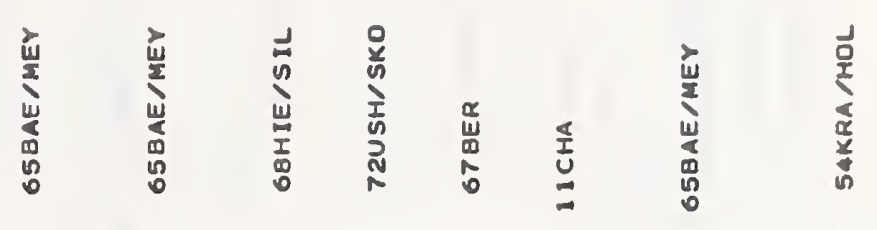

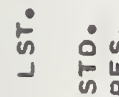

崖

ชับ

ชา

总畜

๕ับ

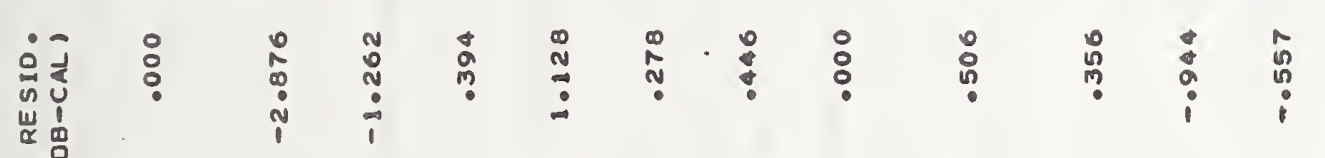

ค.

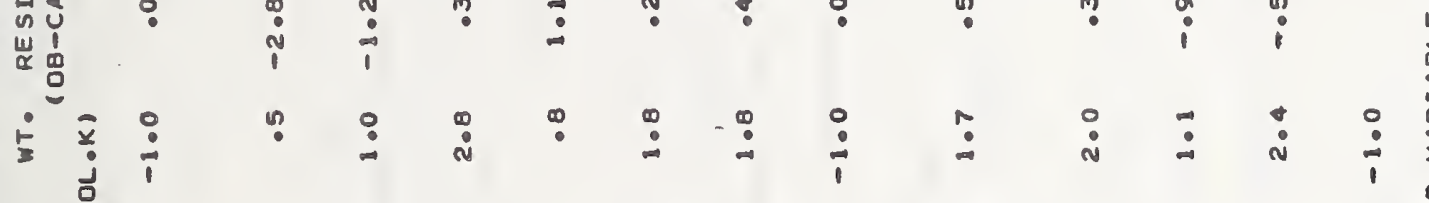

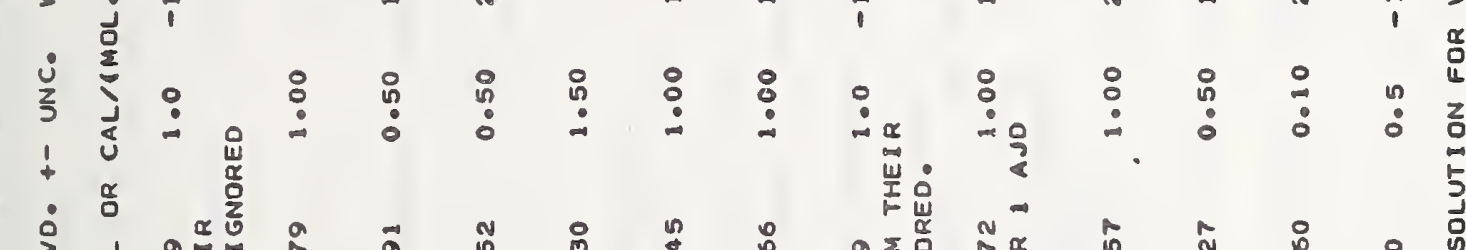

善高

ชั

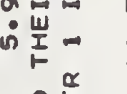

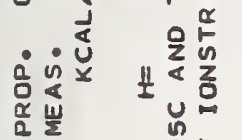
1. 范

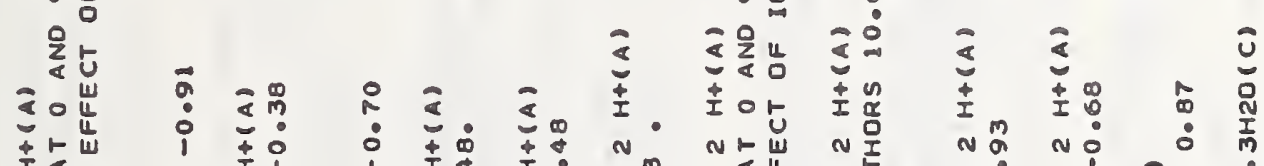

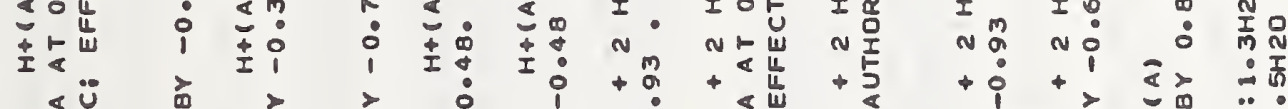

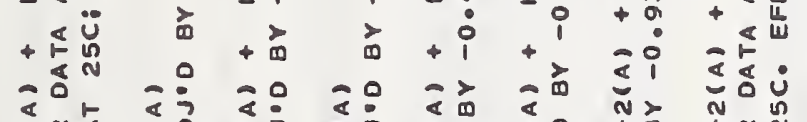

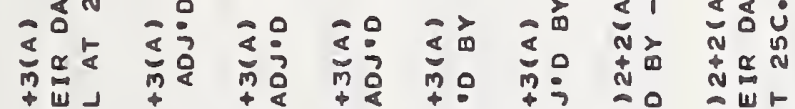

过 吾

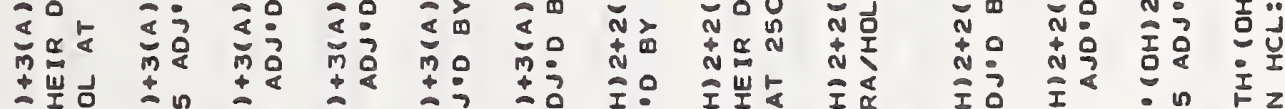

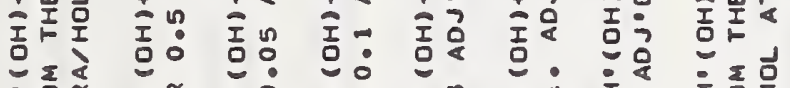

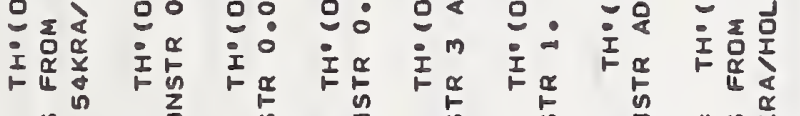

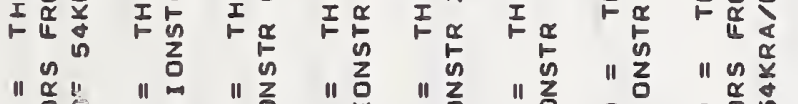

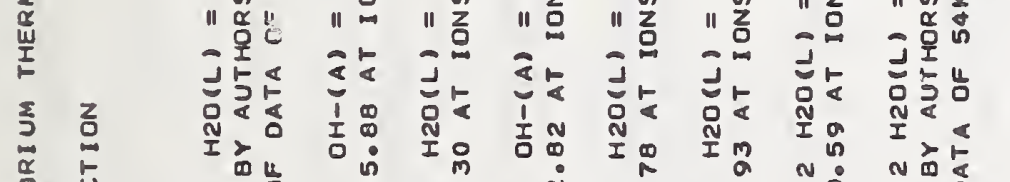

울

㟧

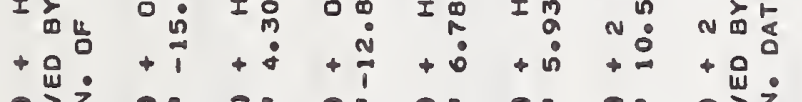

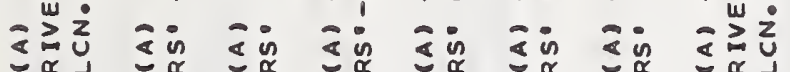

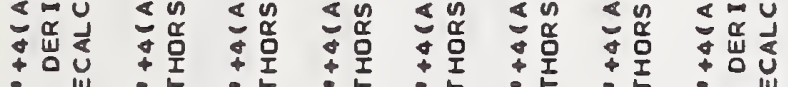

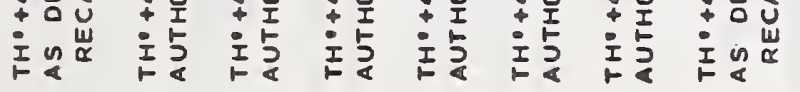

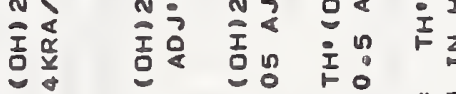

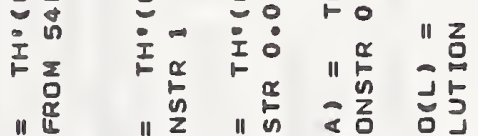

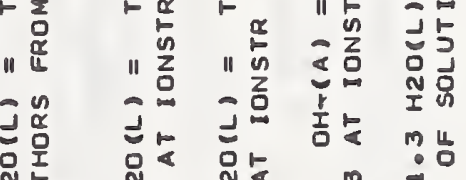

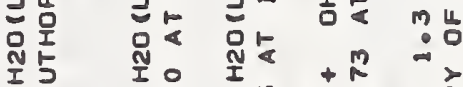

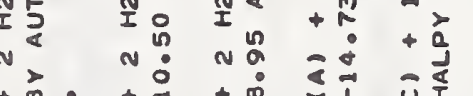

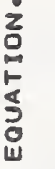

空

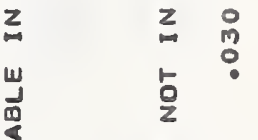

竞:

总

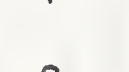




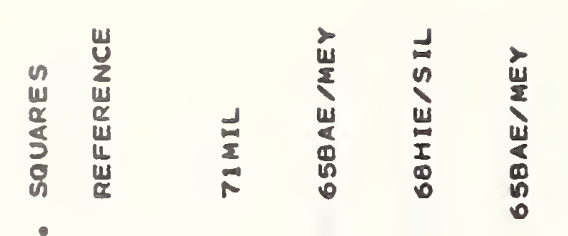

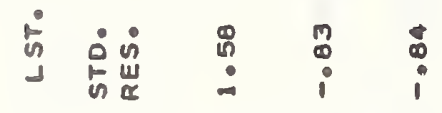

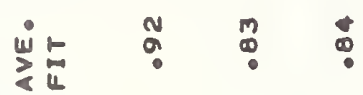

总

点

造离 $\cong \stackrel{N}{\because}$

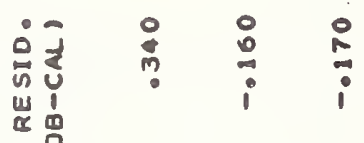

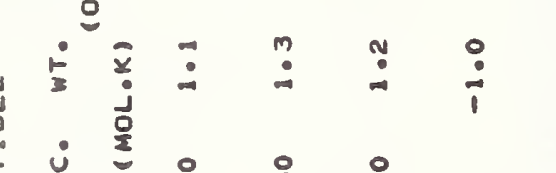

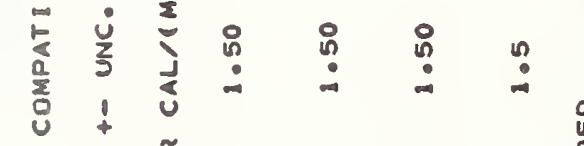

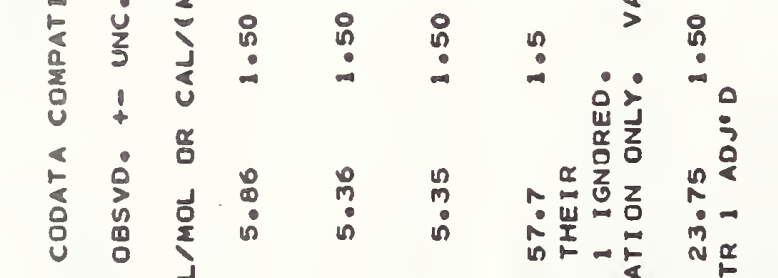

范

ล̊

U⿺⿻一𠃋

(1)

$\therefore$ 过

妾

崖

岁

通

n

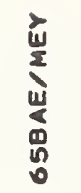

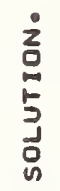

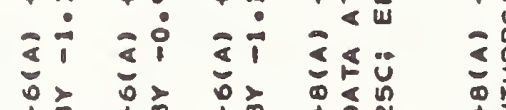

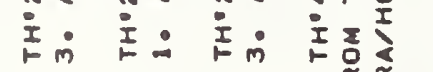

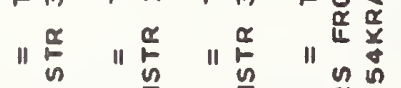

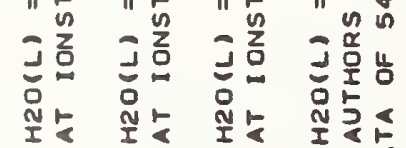

I

11 采

בิ

옹ㅁำ

I点

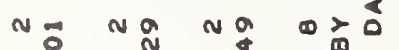

$+2$

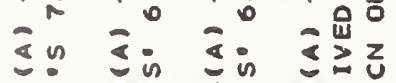

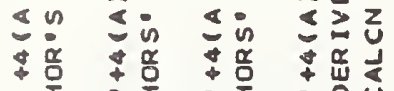

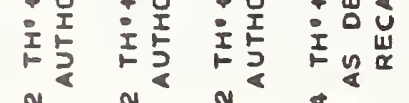

$\leq \dot{0}$

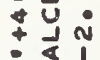

$\frac{1}{n}$
w
$\frac{7}{0}$
0
0

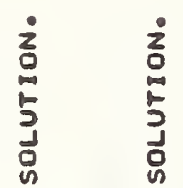

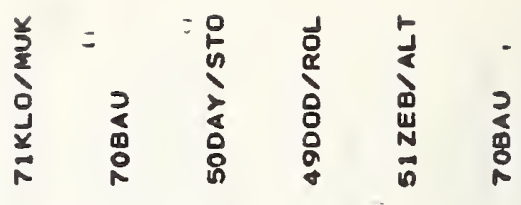

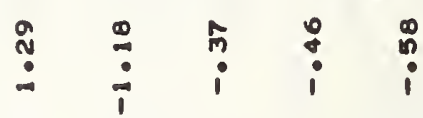

$\stackrel{0}{\circ}: \stackrel{0}{:}: \stackrel{?}{:}$

$\cong \cong \cong \cong$

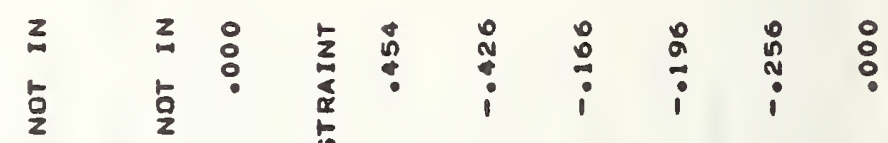

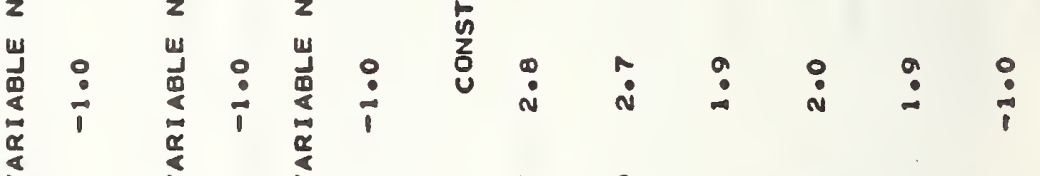

:

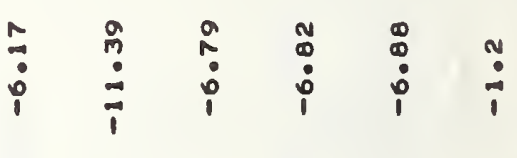

แू⿺辶⿻

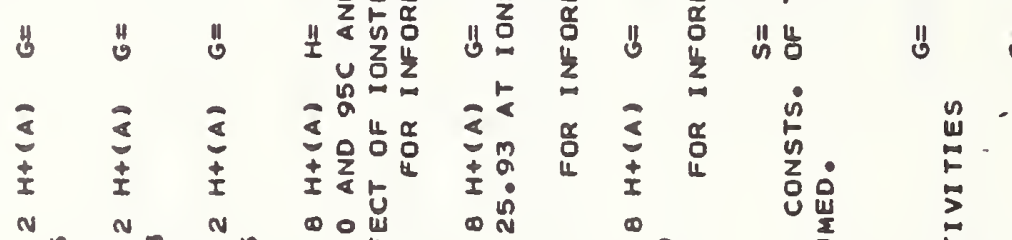

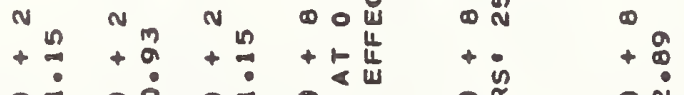

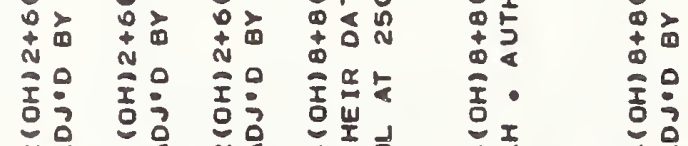

施

in

11.

د

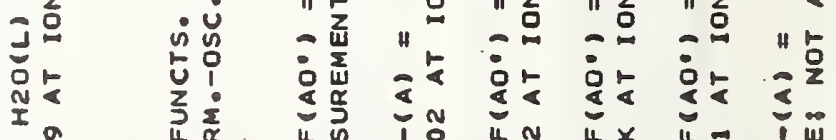

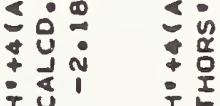

i is

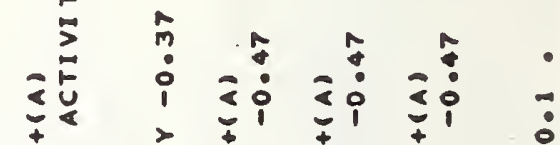

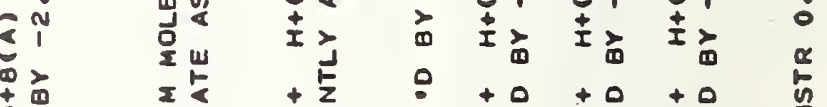

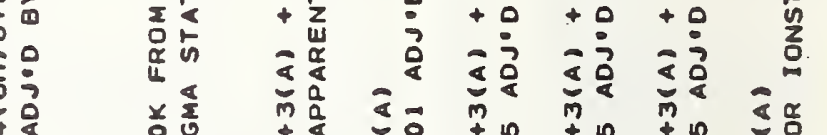

क ?

$+\infty$

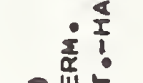

tu mo to

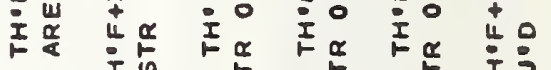

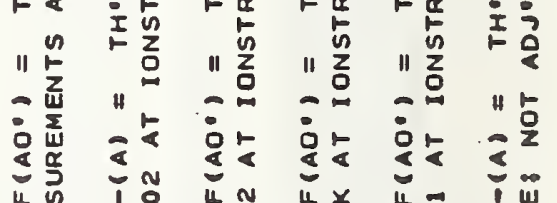

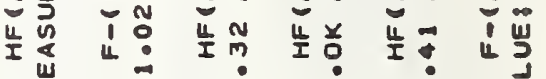

可㟧

$+\frac{w}{2}+\vec{i}+\dot{i}+\dot{i}+\dot{i}+\frac{\dot{p}}{5}$

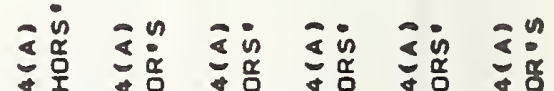

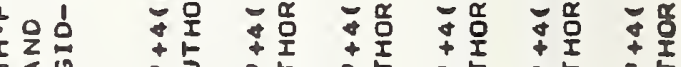

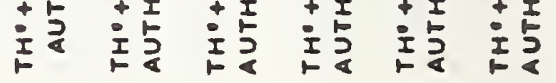




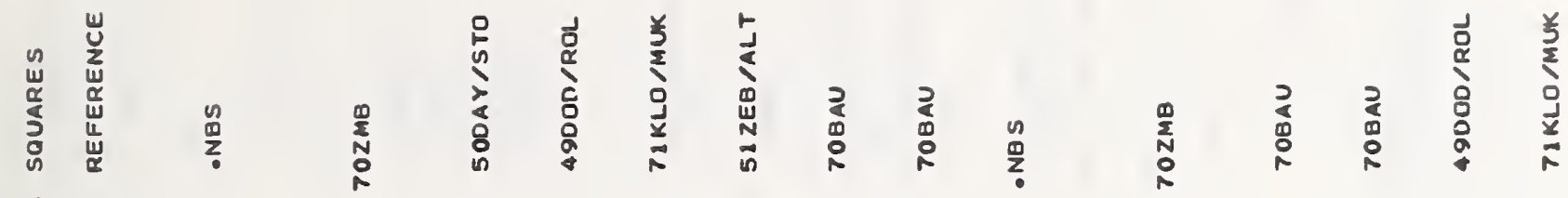

容

憝

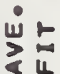

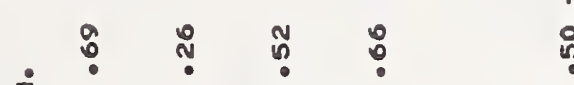

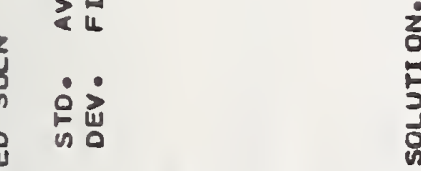

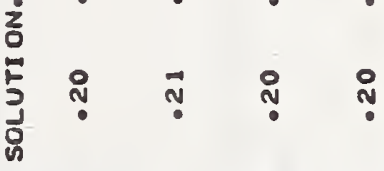

$\begin{array}{lll}i & 2 \\ 0 & 0 \\ 5 & 5 \\ 0 & 0 \\ 0 & 0 \\ 0 & 0\end{array}$

กั

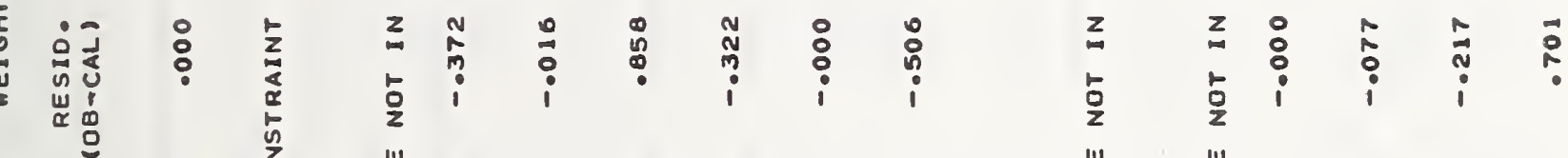

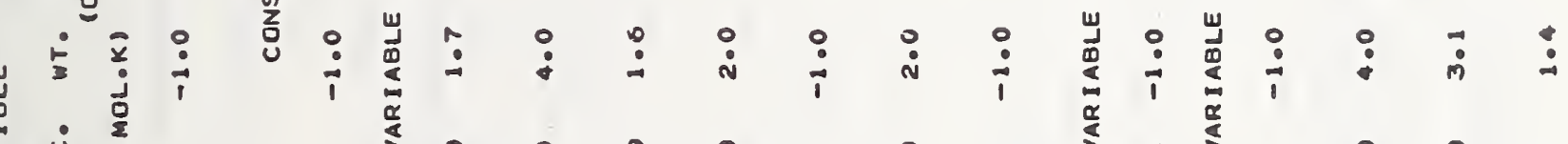

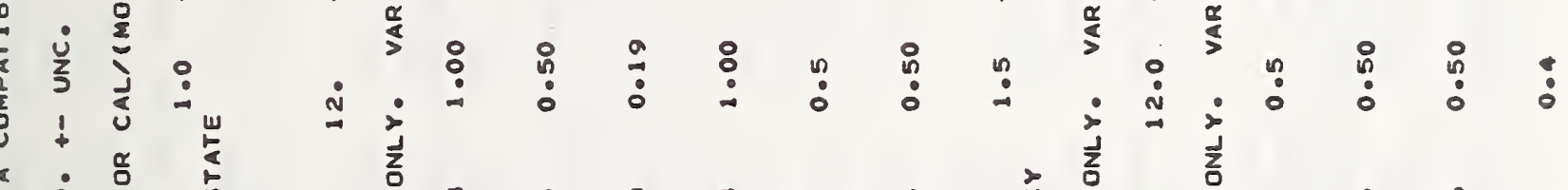

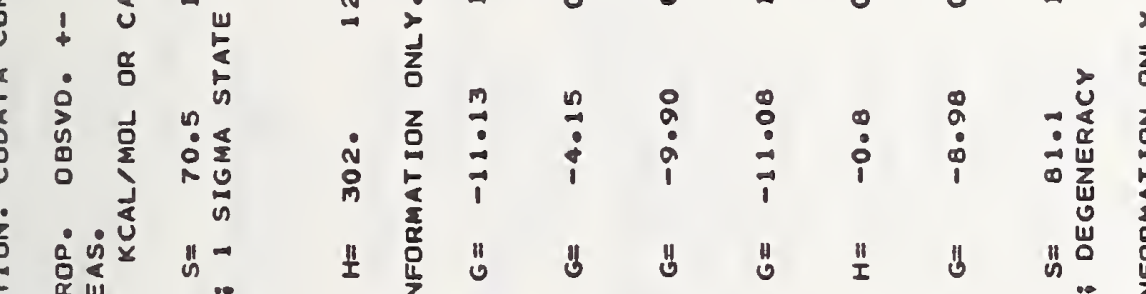

西

s

音

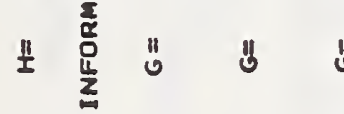

운

出

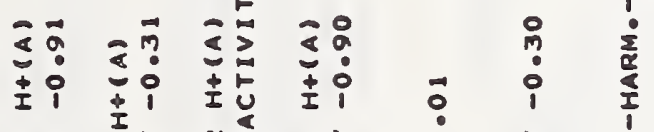

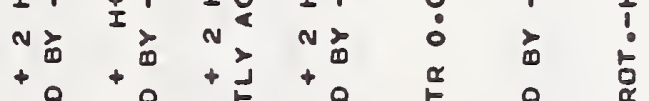

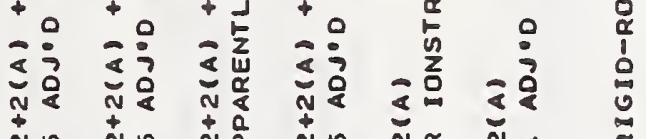

ก! 每

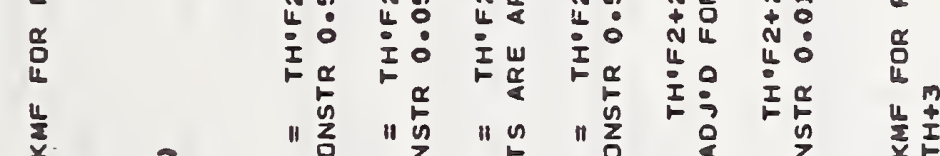

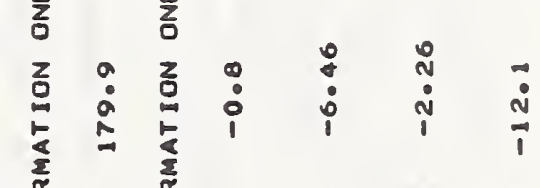
U⿺

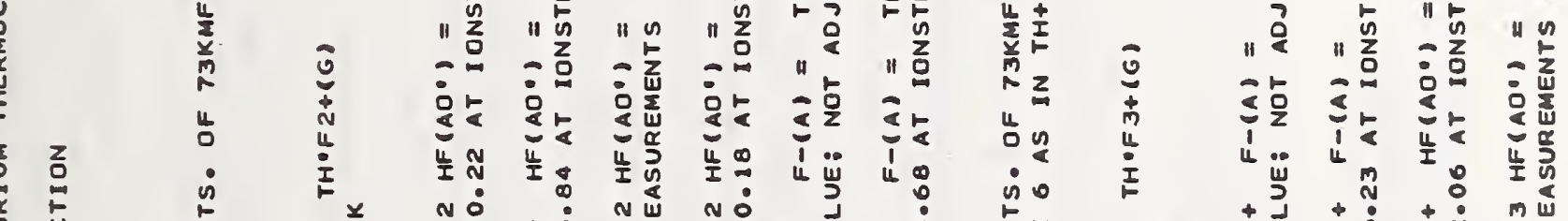

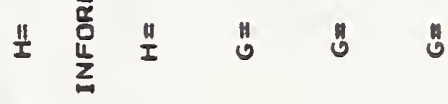

品

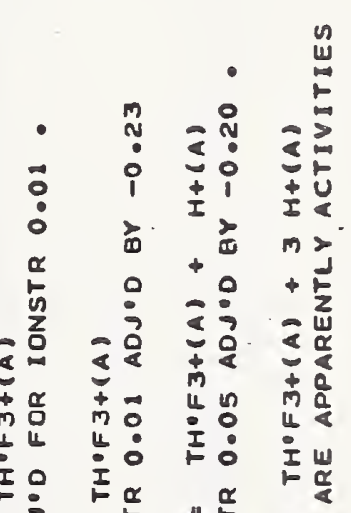

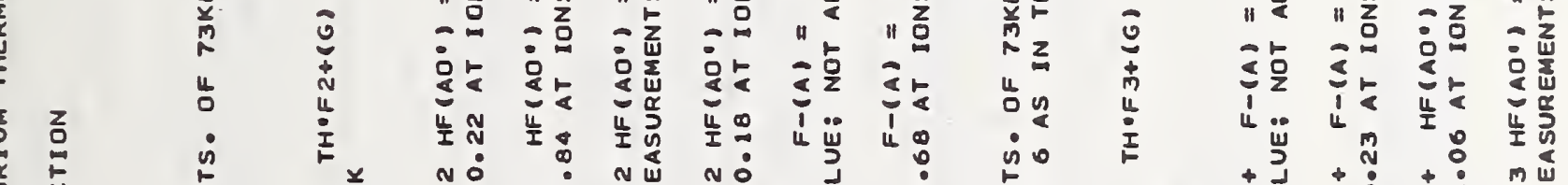

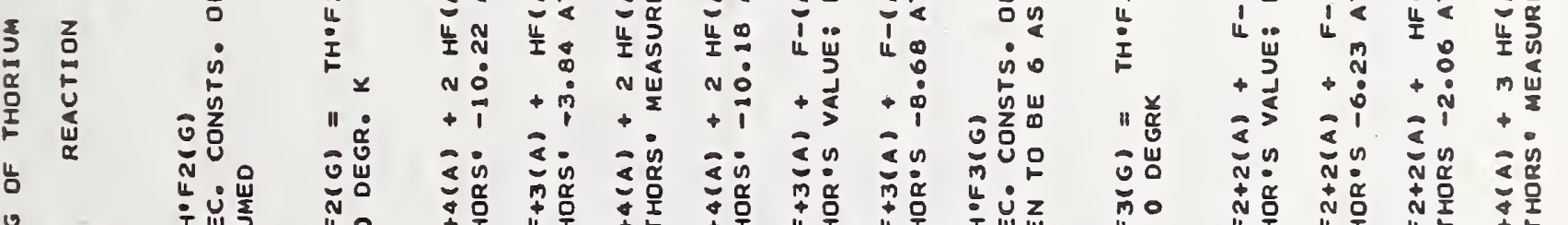

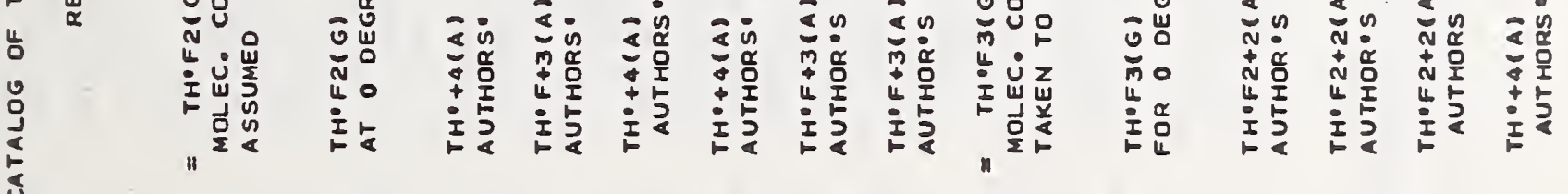

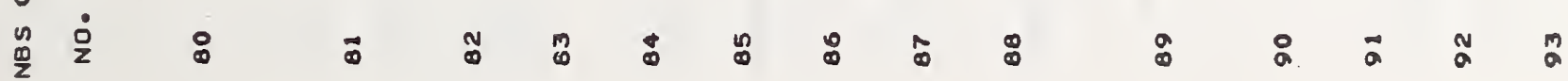




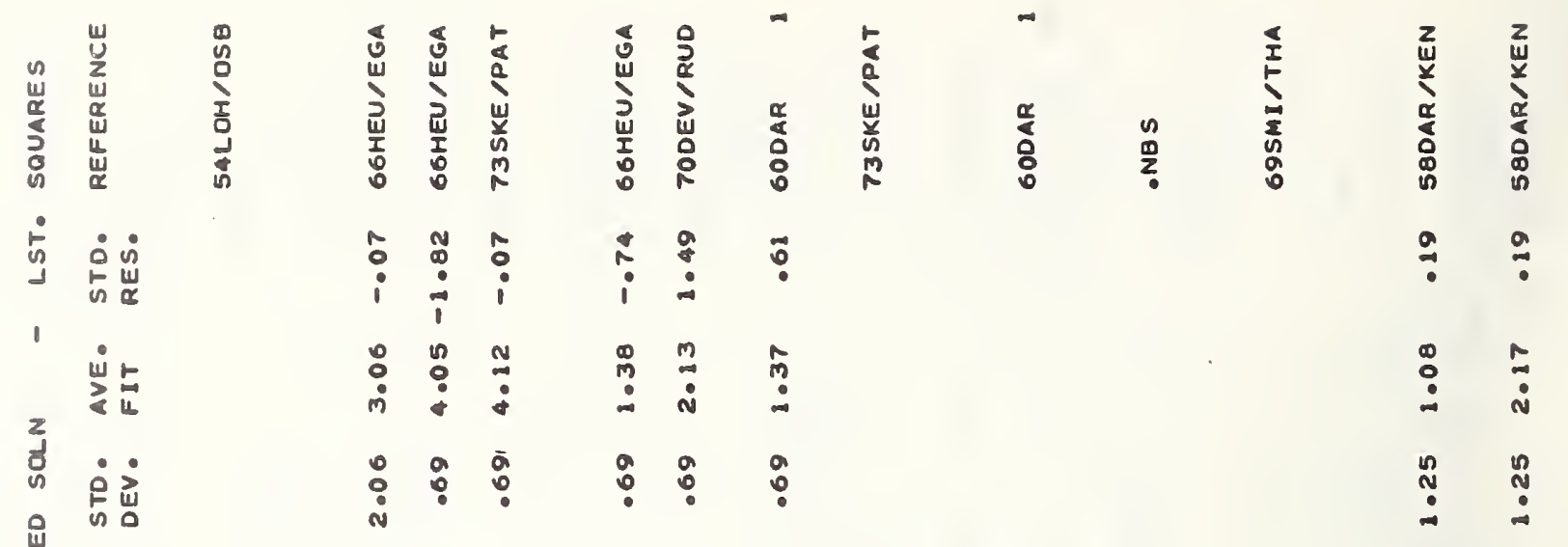

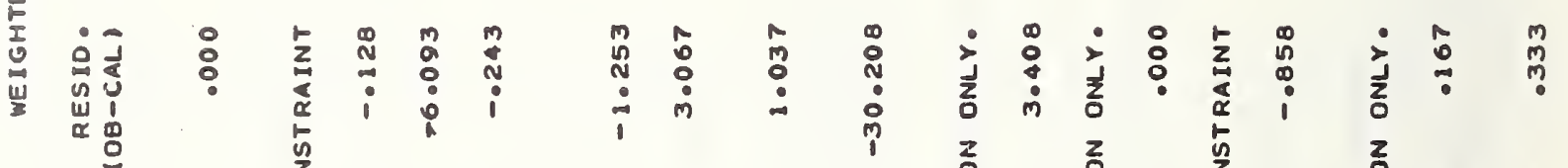

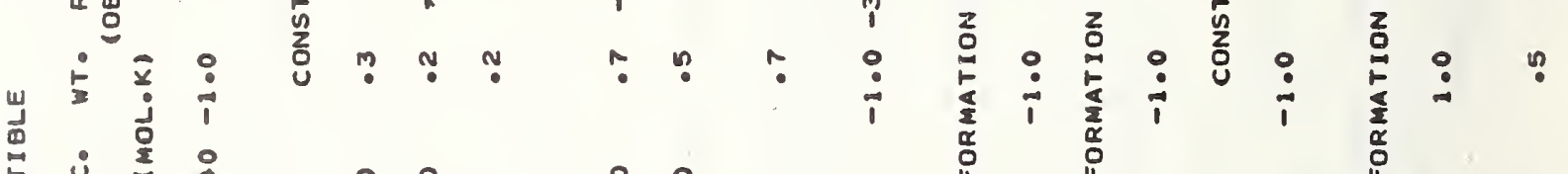
ไู)

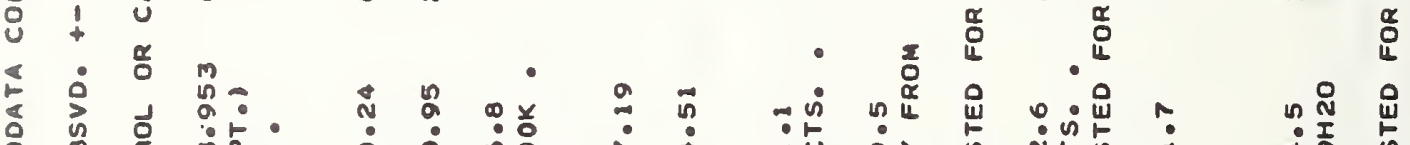

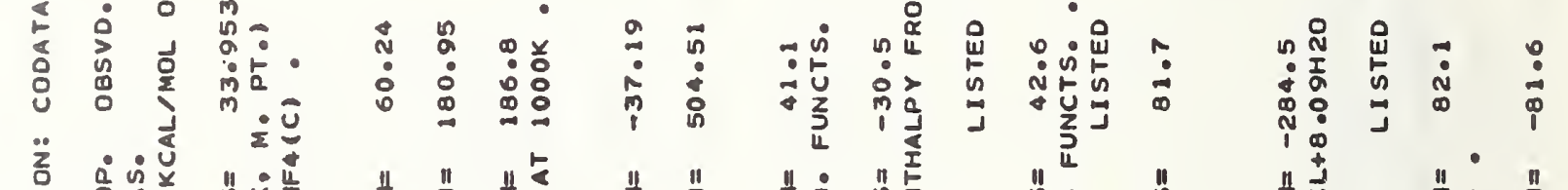

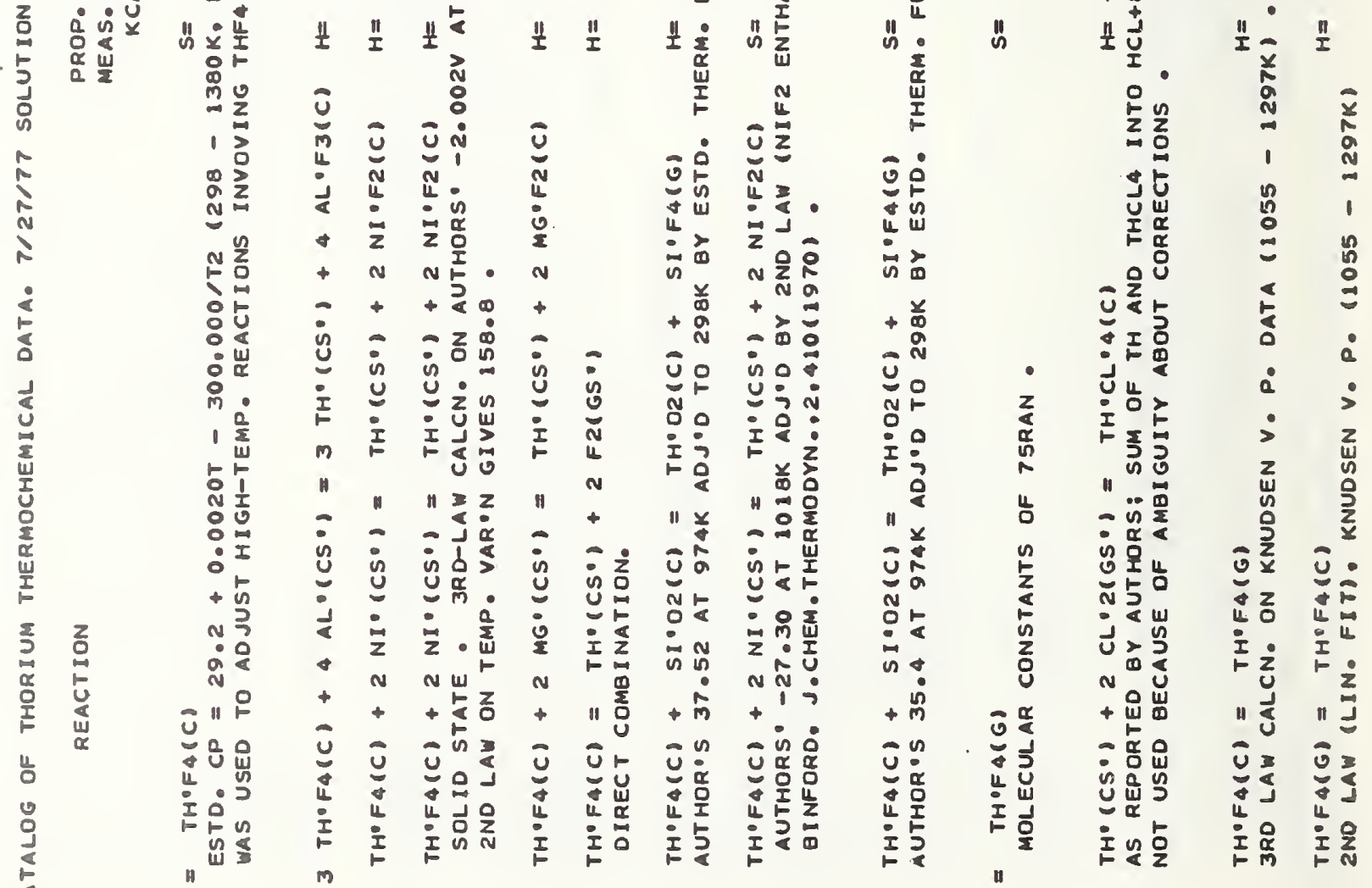

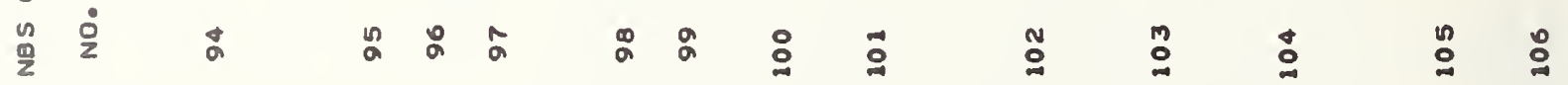




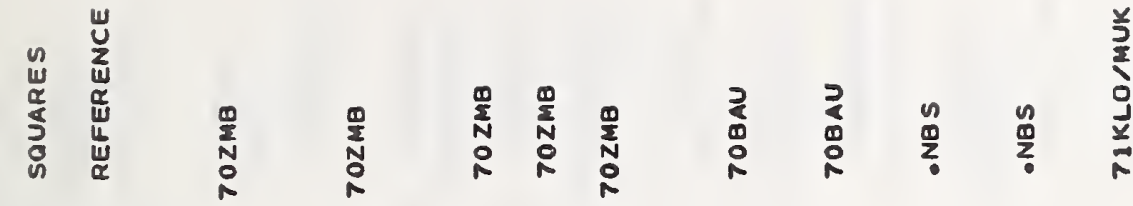

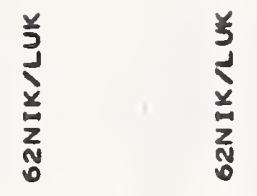

占

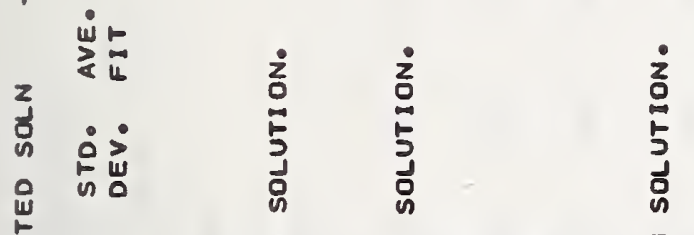

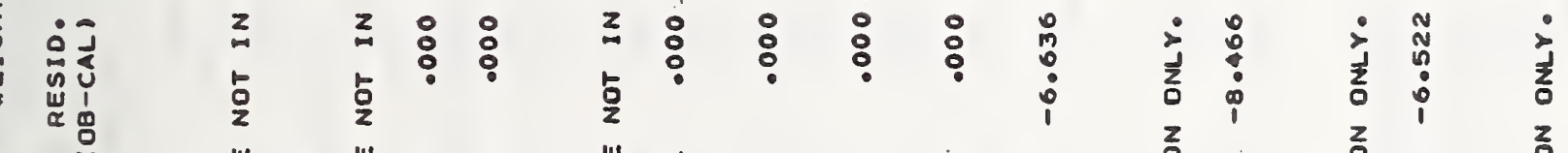

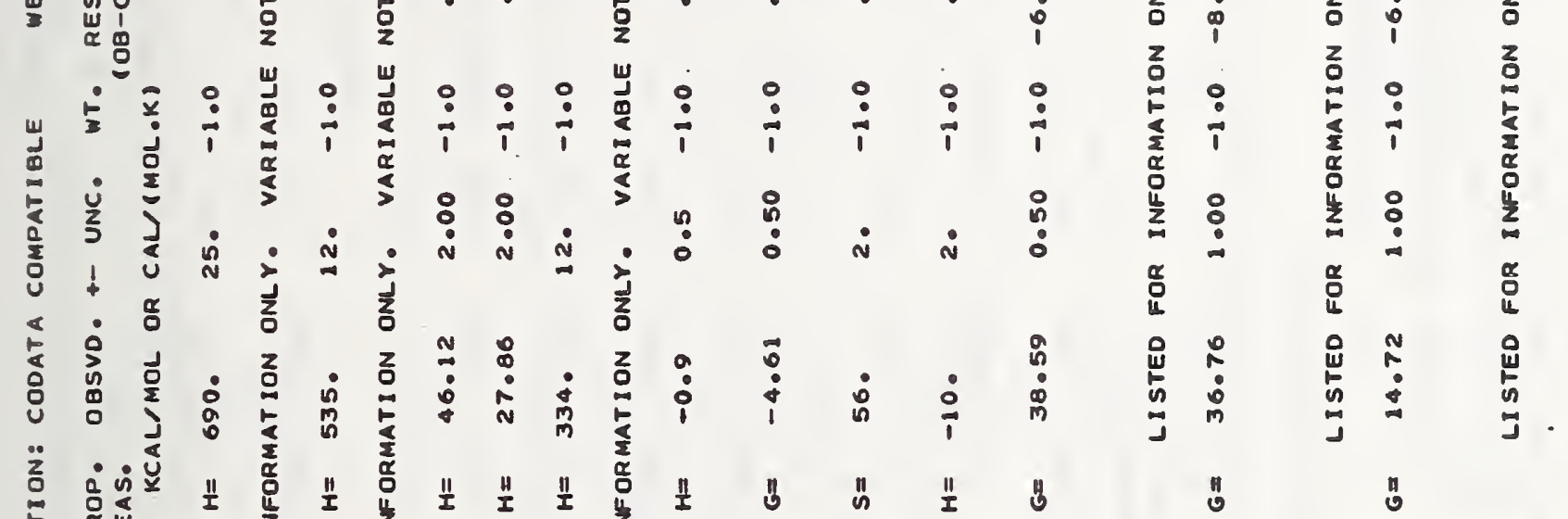

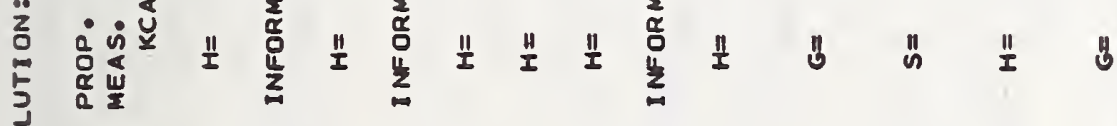

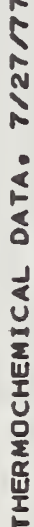

然哭

哭

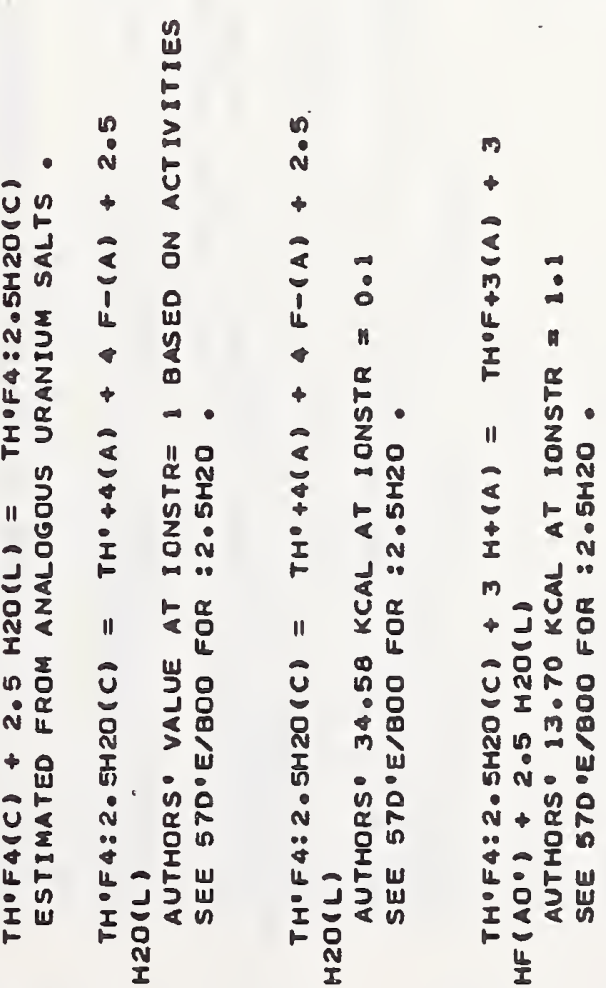

台完 


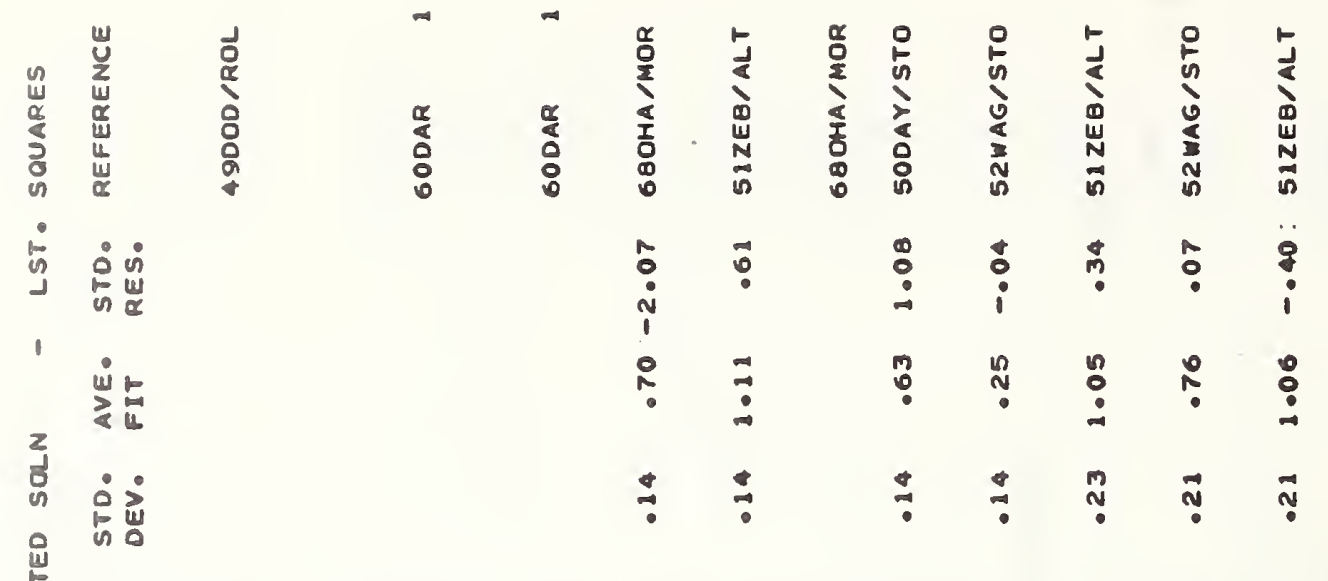




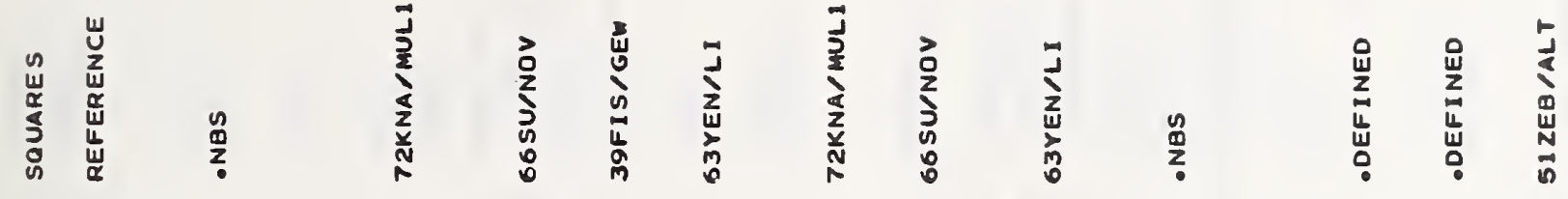

嵩紊

is

$\stackrel{0}{\circ}$

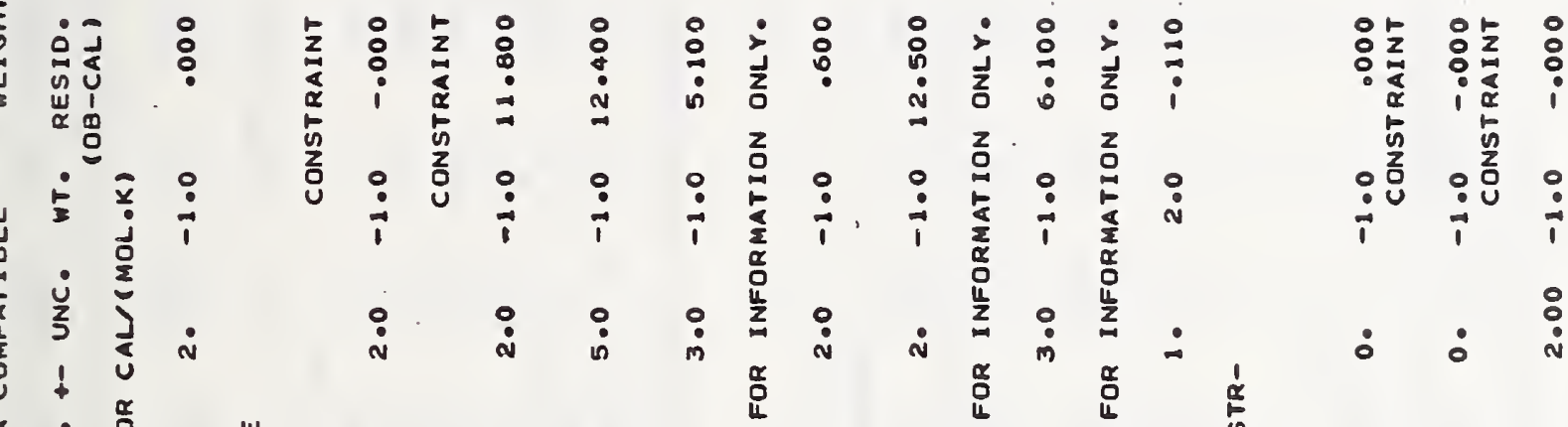

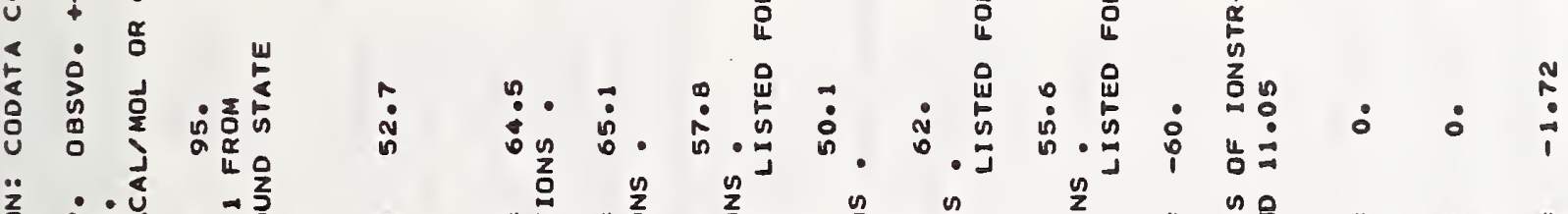

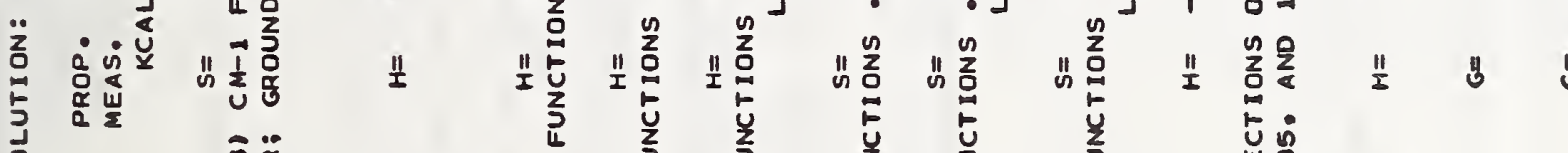

in

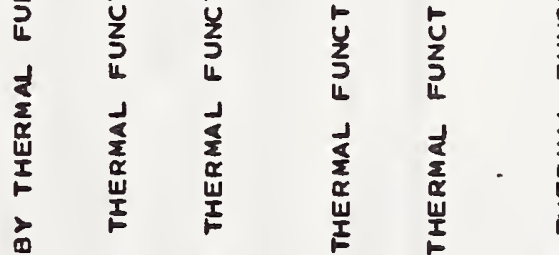

m

ชิธ

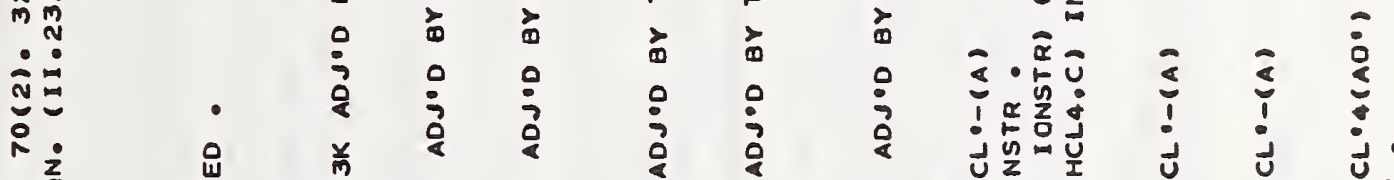

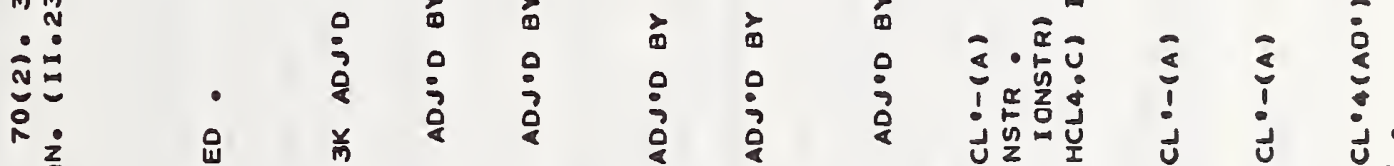

○公主

$>2$

造

₹

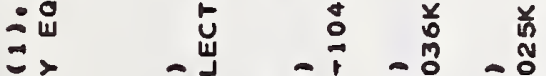

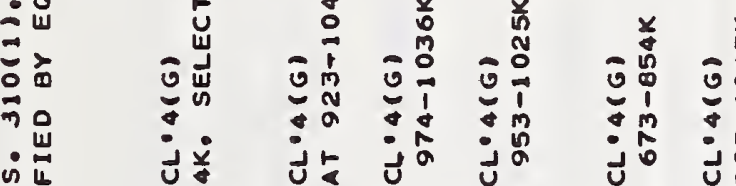

din

$\div \frac{1}{1}$

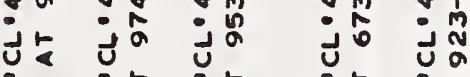

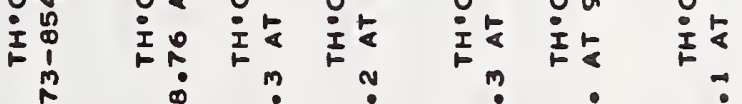

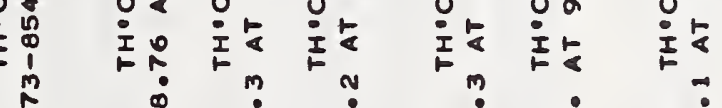

递

-200
+025

岂:

岃:

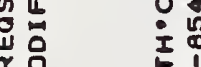

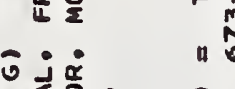

U ش

$11 \dot{0}$

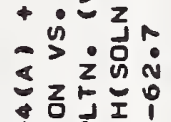

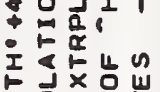

西希出

น 造造娄

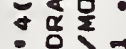

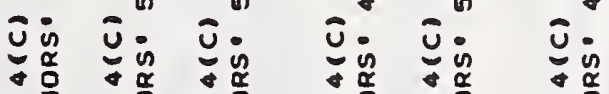

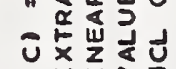

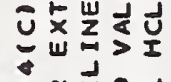

ปั่

움

औ

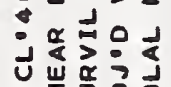

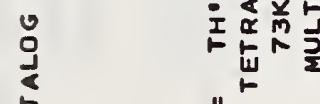

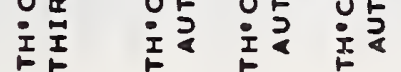

ป๋

บำ

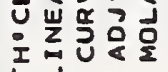

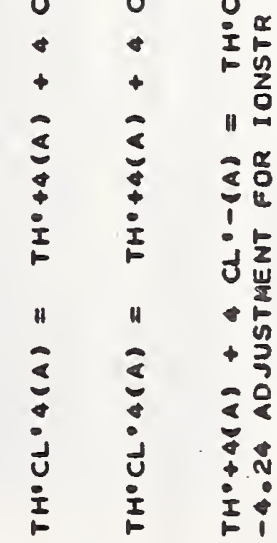

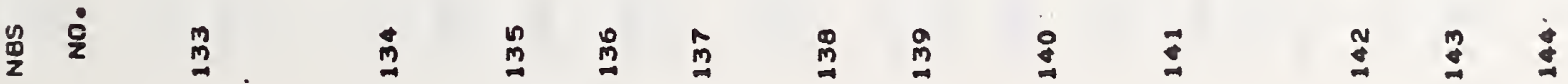




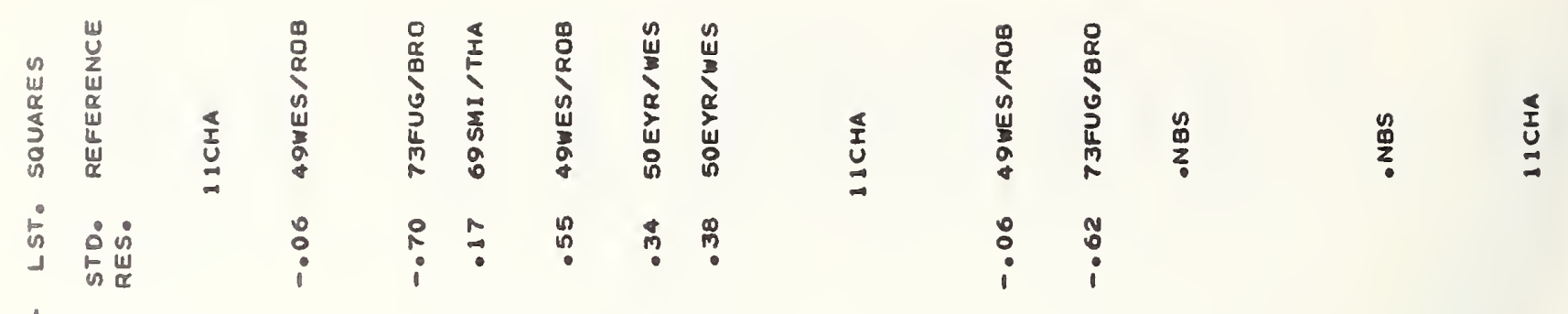

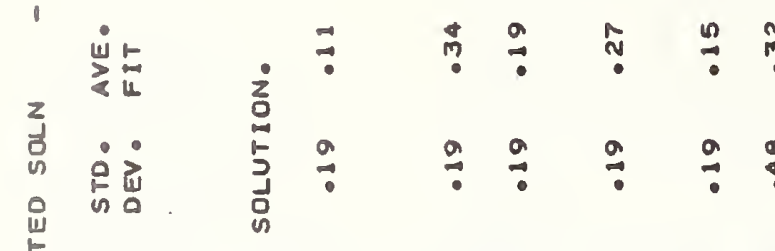

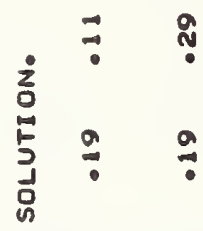

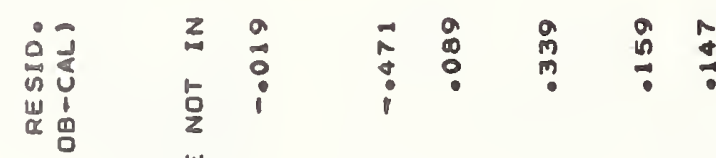

草

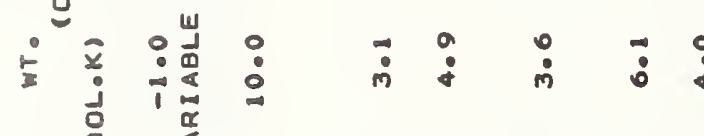

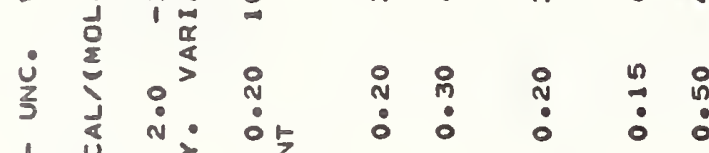

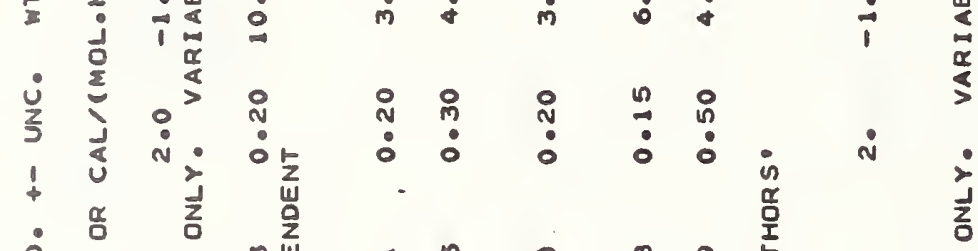

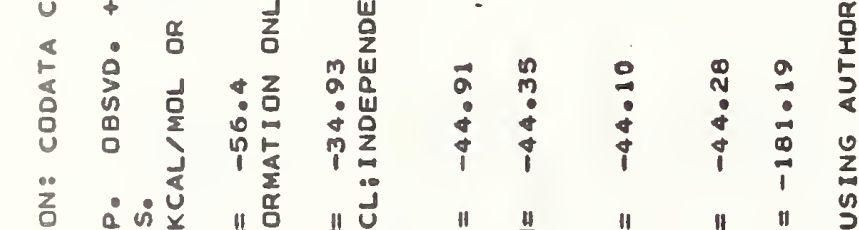

(1)

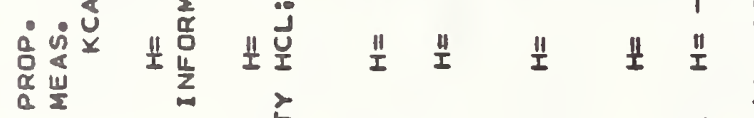

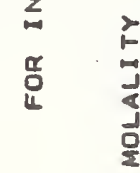

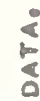

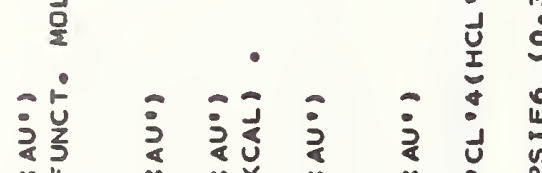

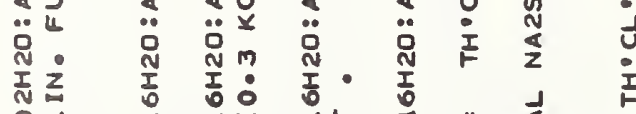

通

:」.

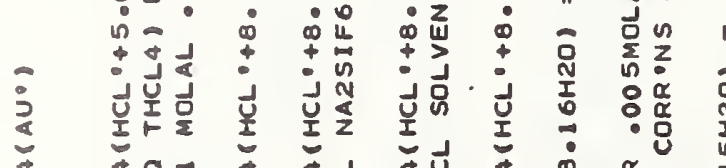

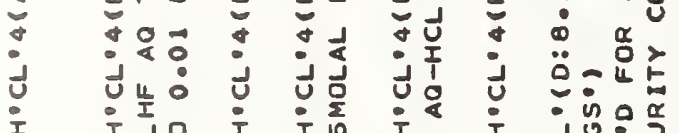

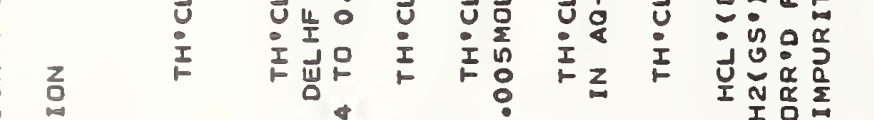

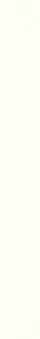

政

$y$

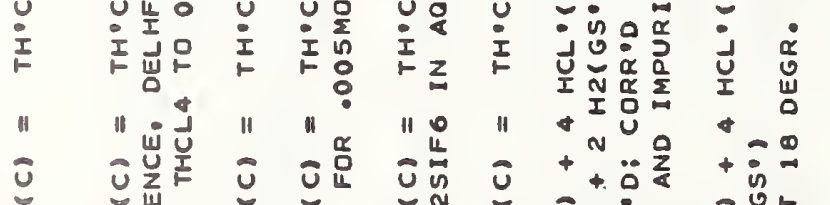

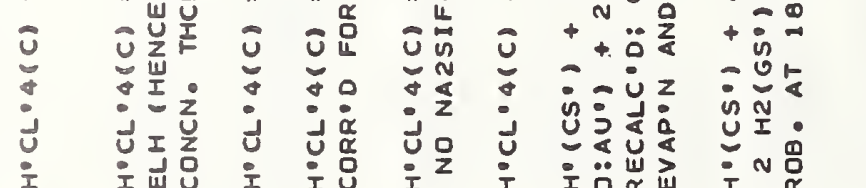

药

క

然

高完

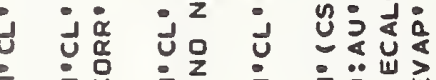

:

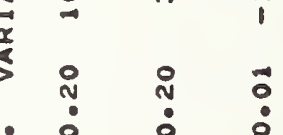

光 000

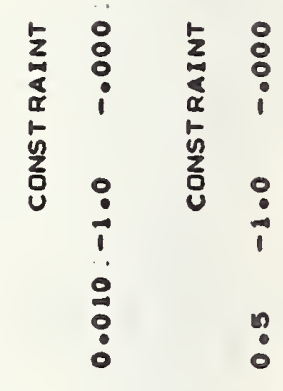

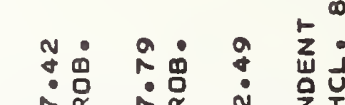




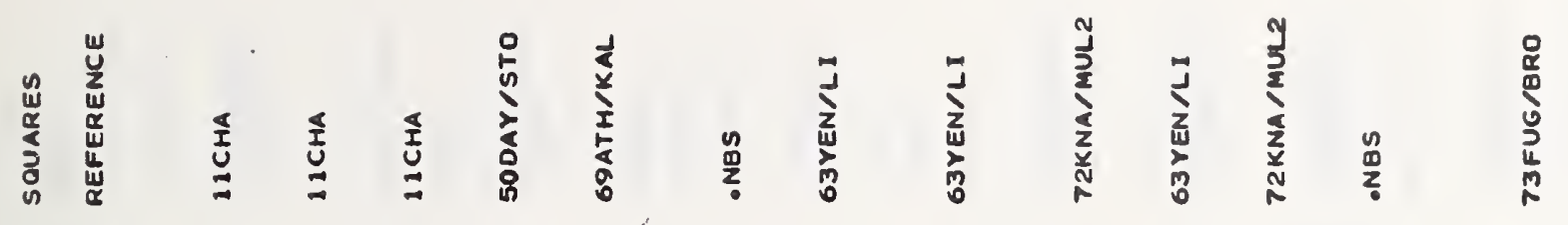

亨旁兽

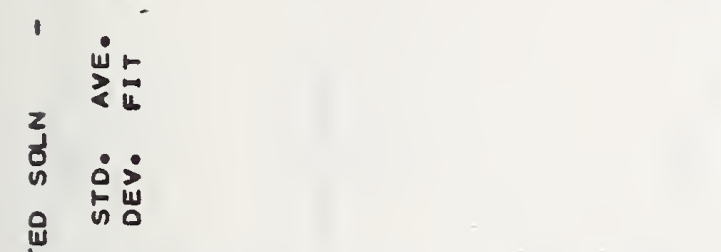

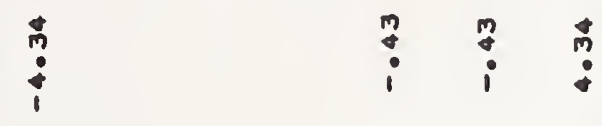

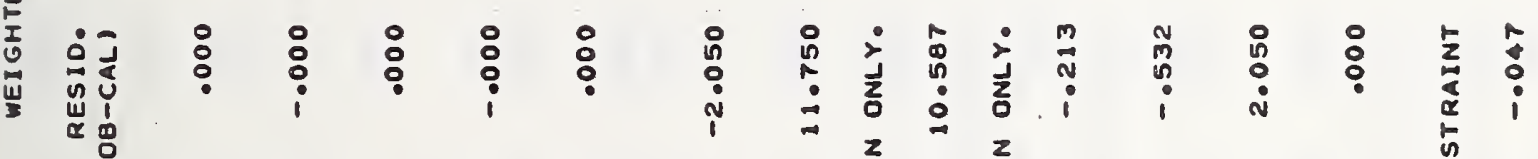

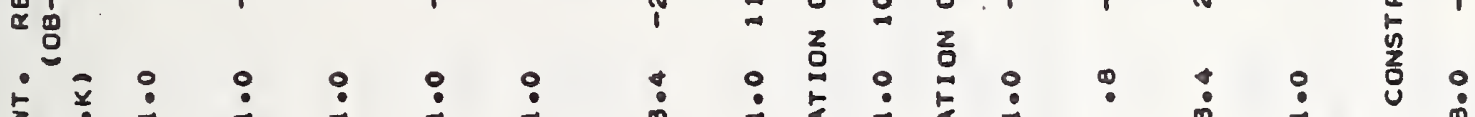

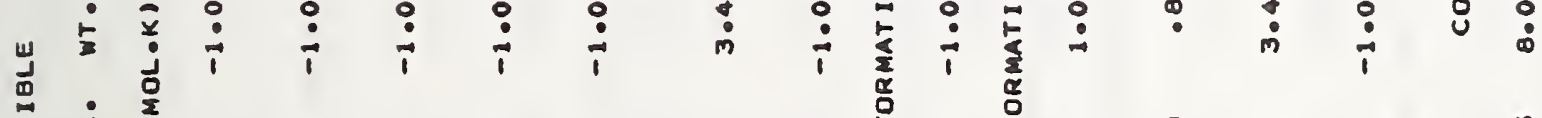

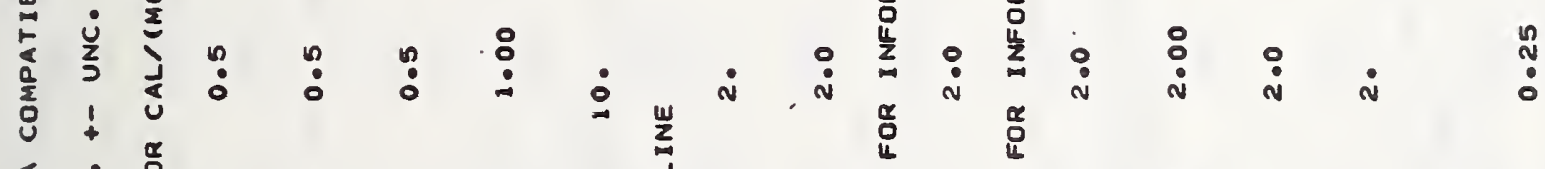

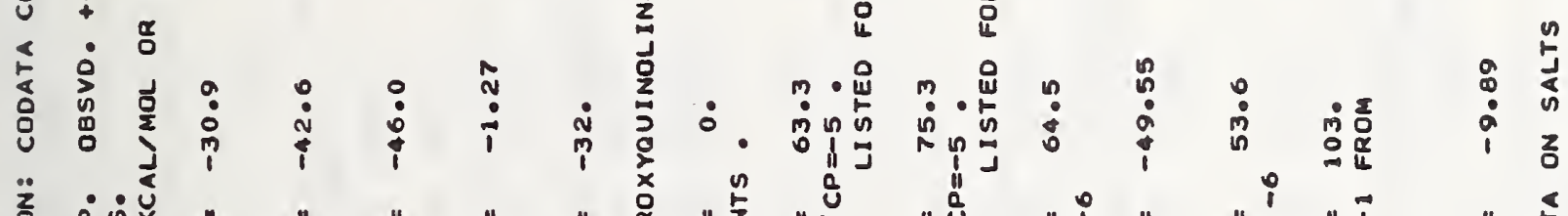

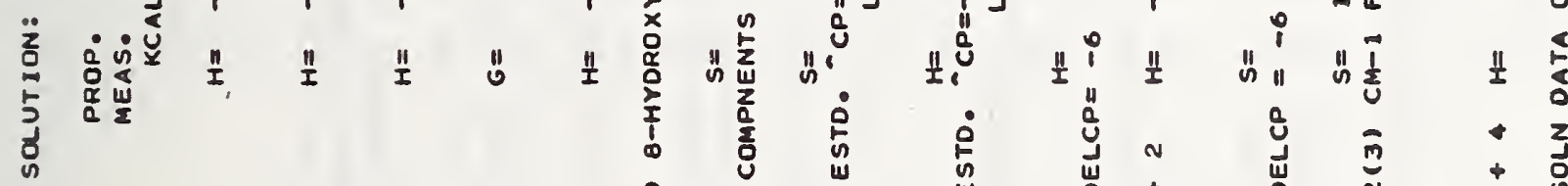

s

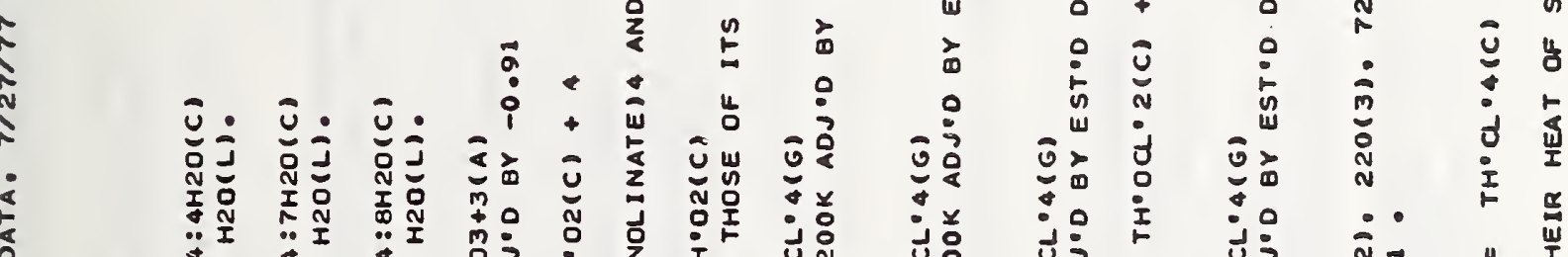

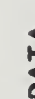

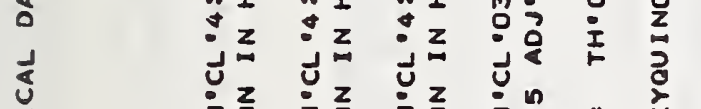

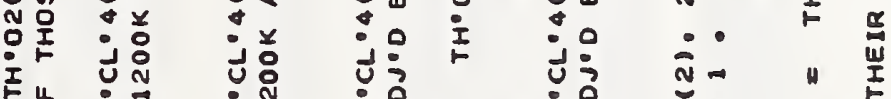

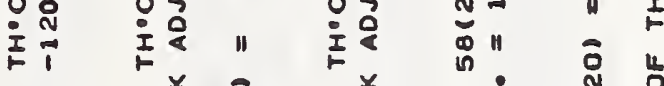

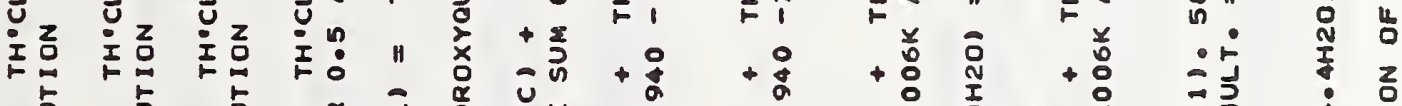

"

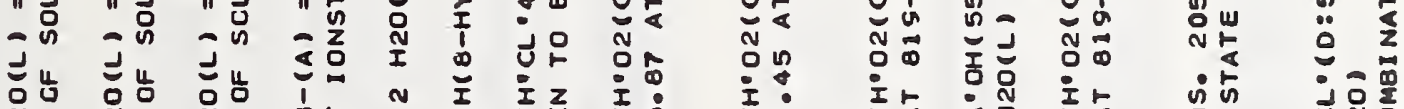

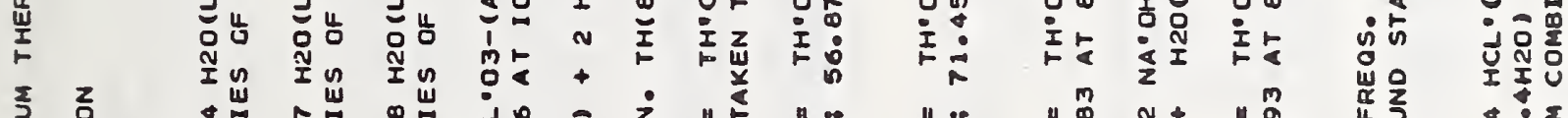

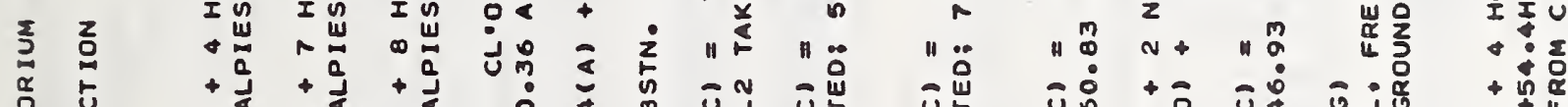

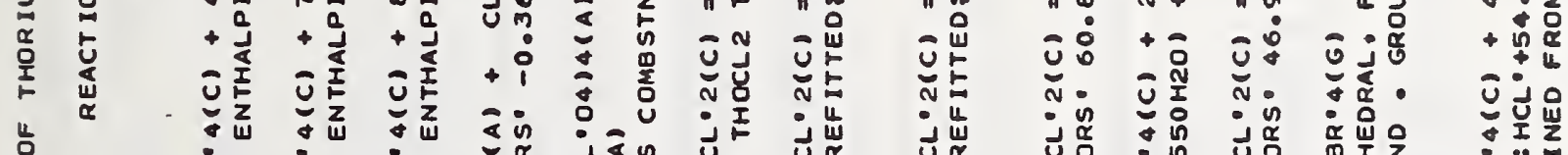

눙

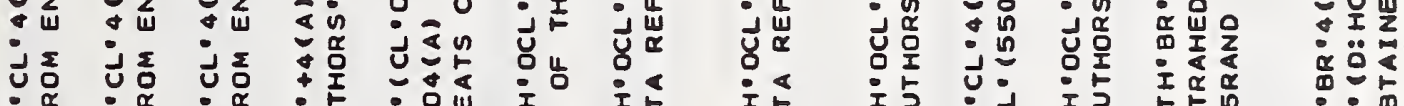

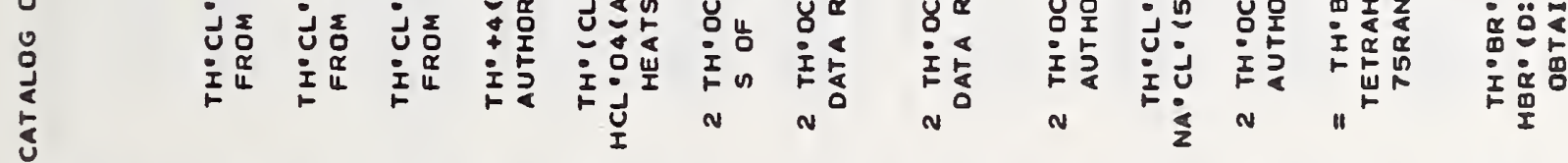

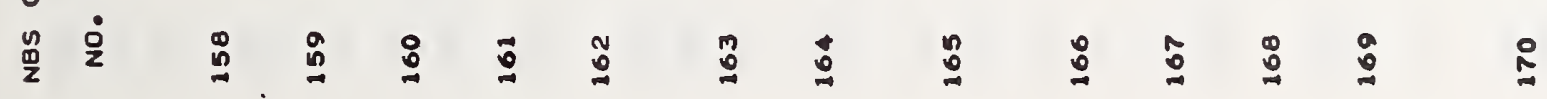



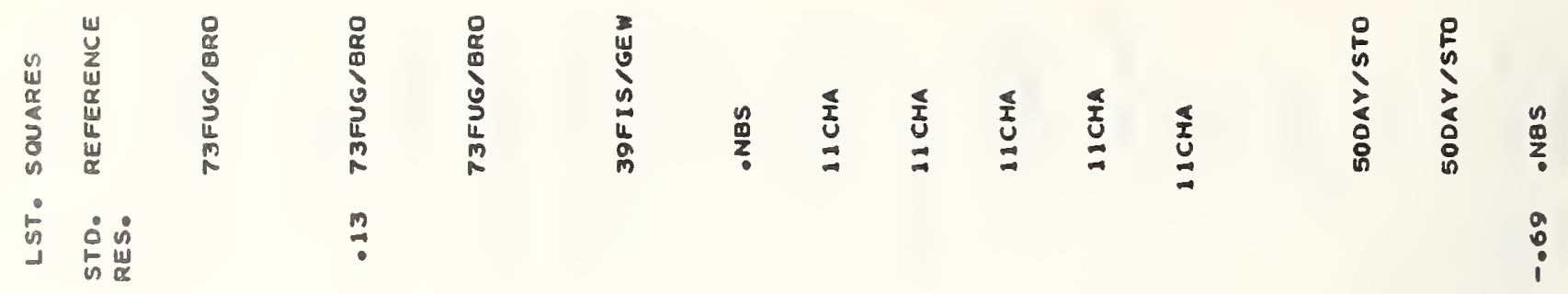

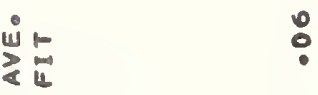

它焉

总员

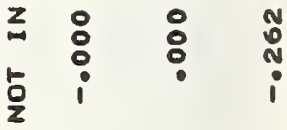

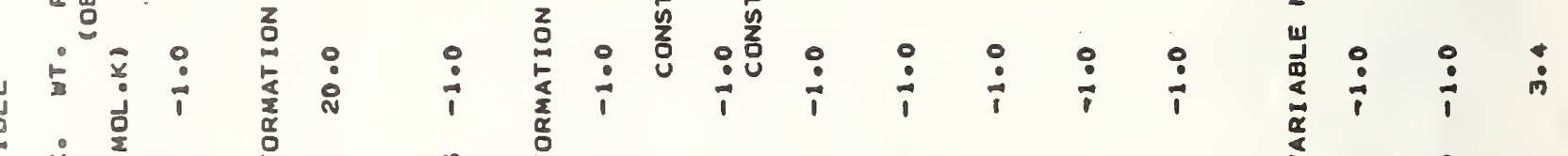

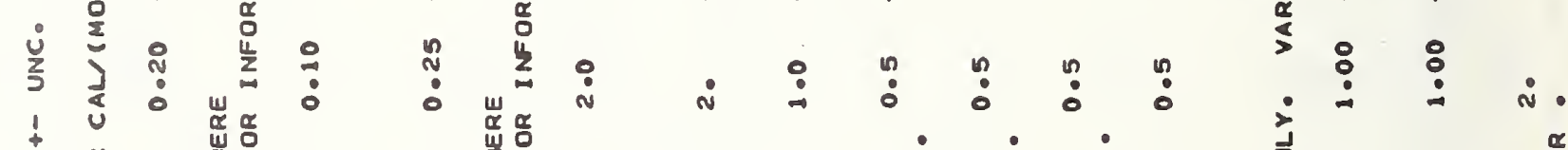

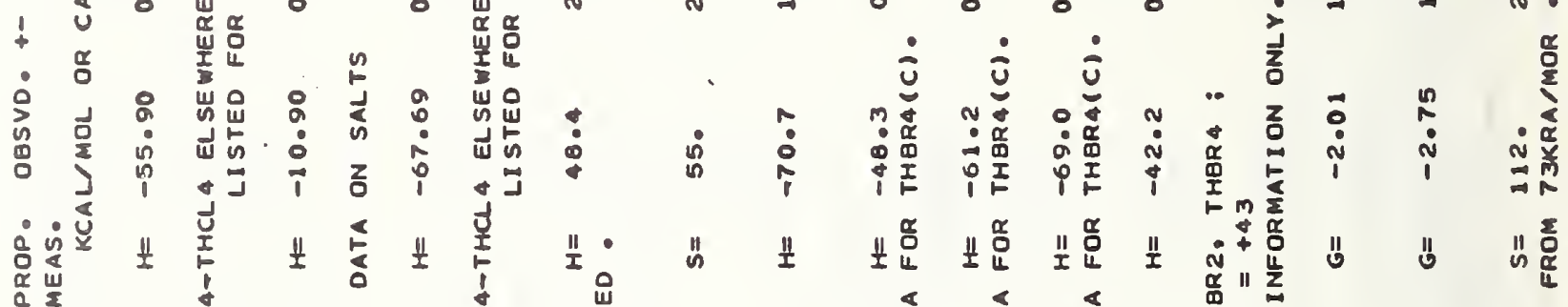

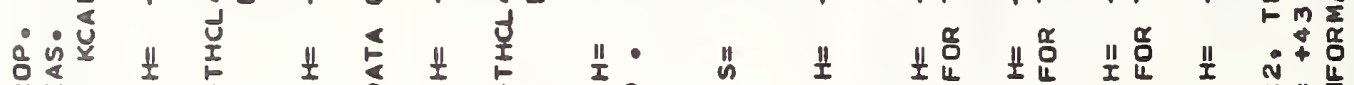

范

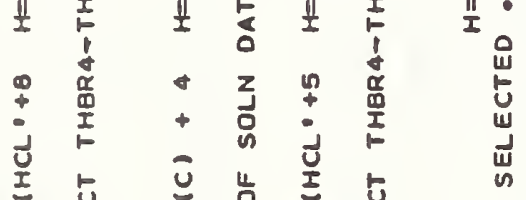

s

s

$+\frac{2}{+2}$

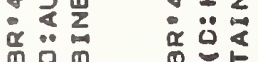

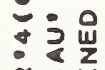

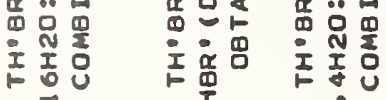

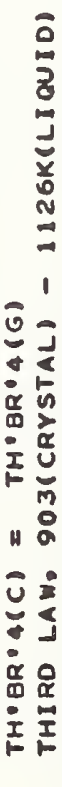

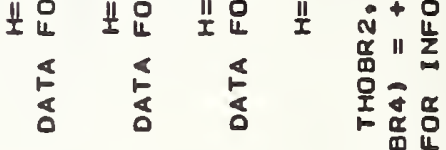

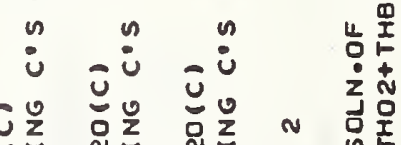

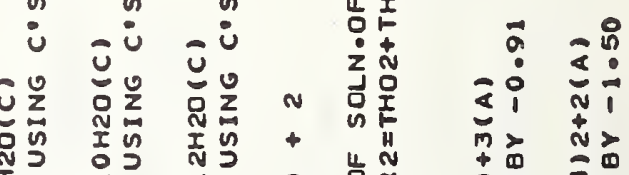

孚 0 J

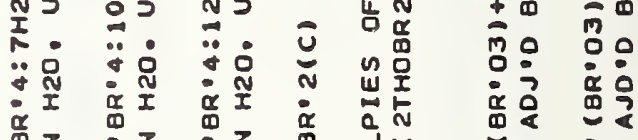

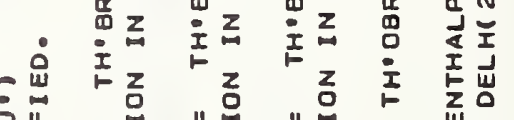

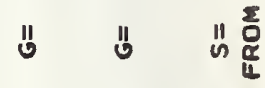

选

盟

$\stackrel{2}{2} \cong$

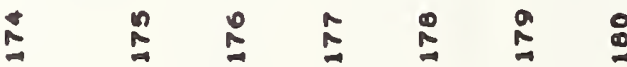

ฮั 


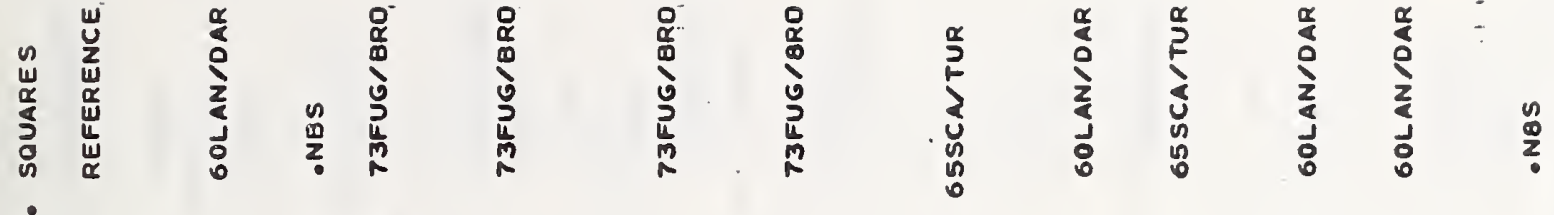

高旁尊

(1)

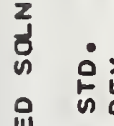

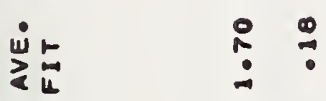

竎高

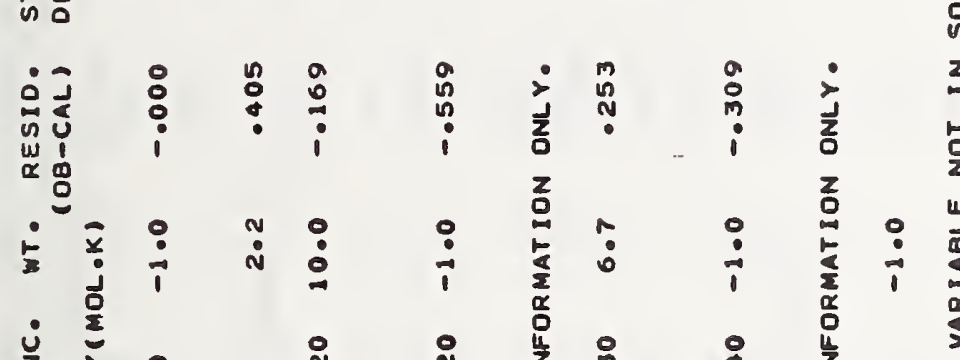

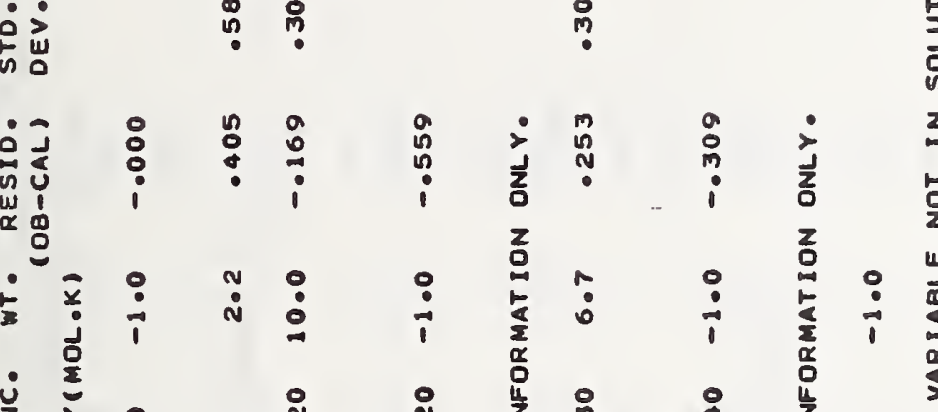

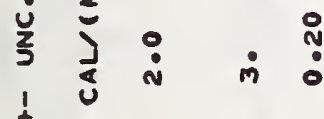

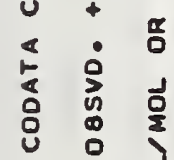

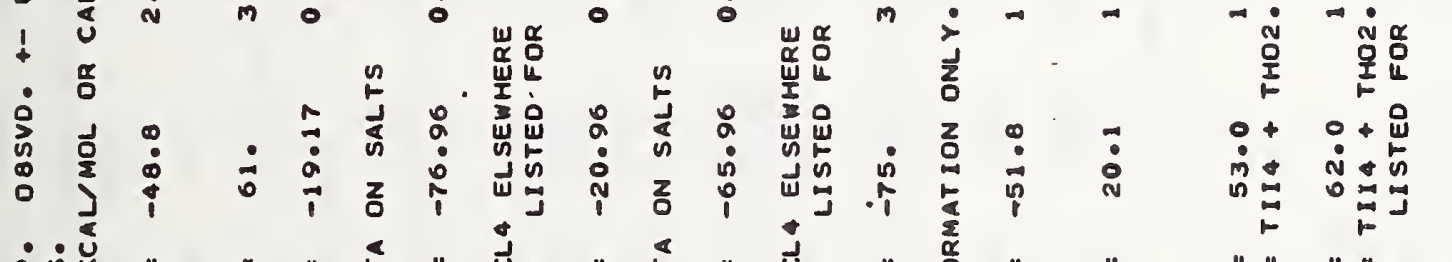

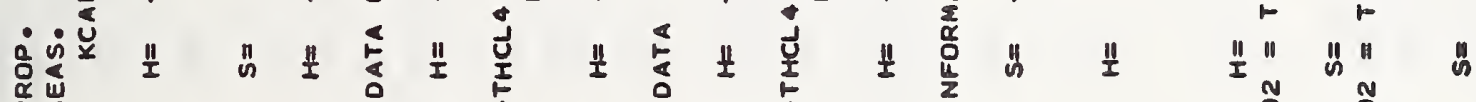

:

م

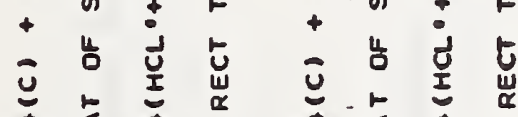

†

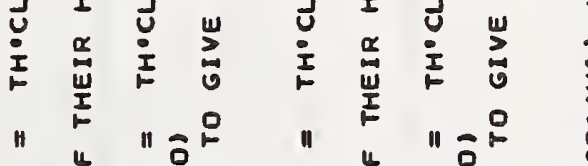

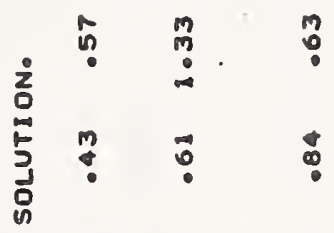

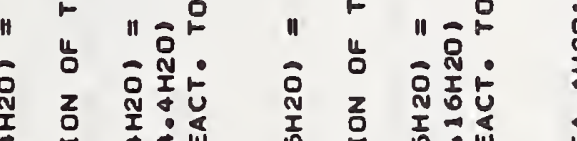

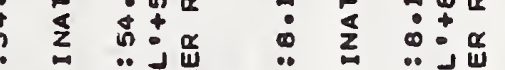

Uิ

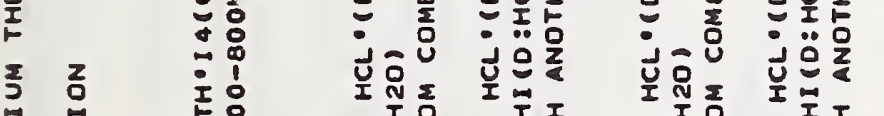

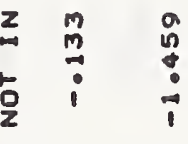

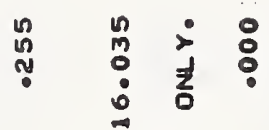

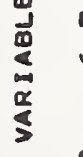




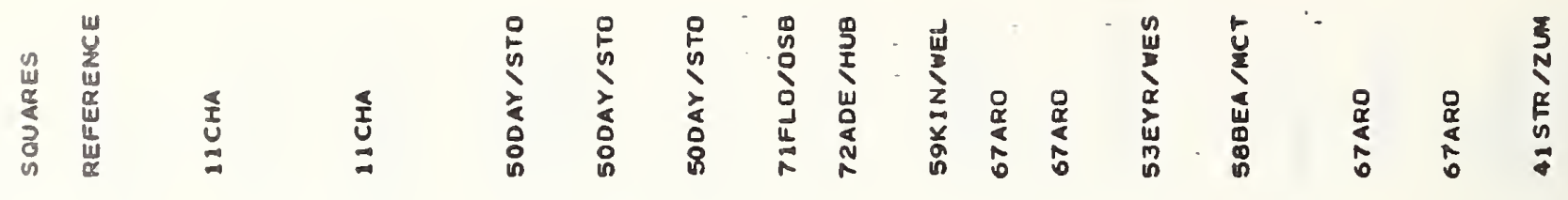

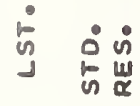

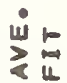

家㟧

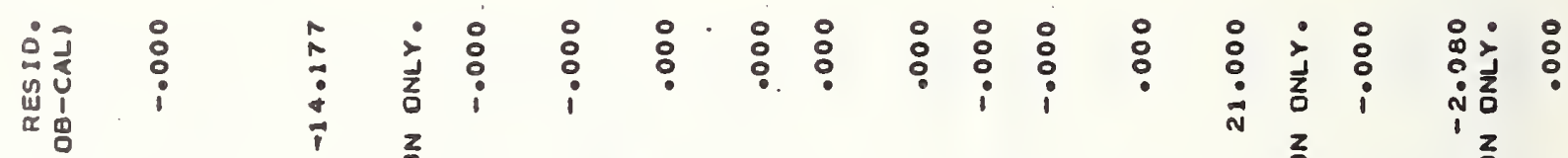

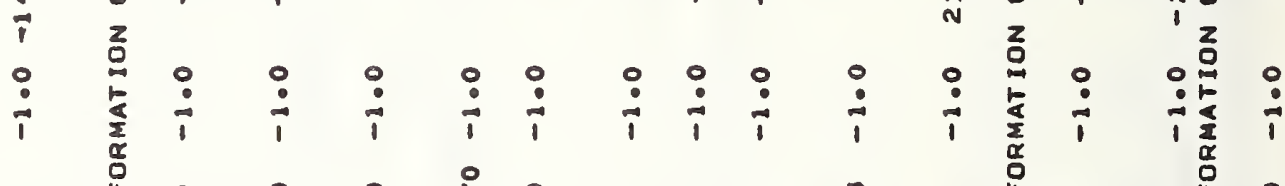

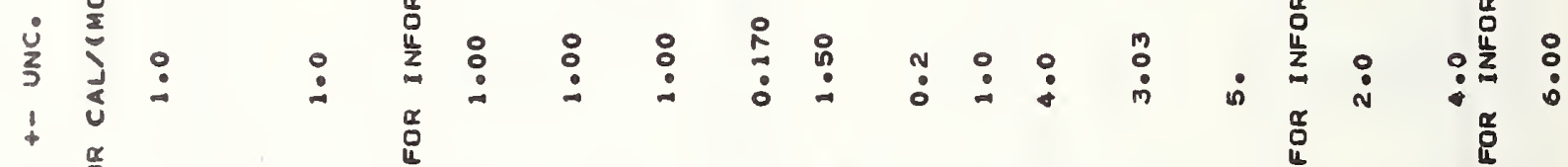
$\varangle \quad$ :

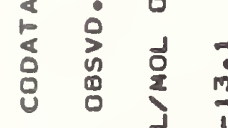

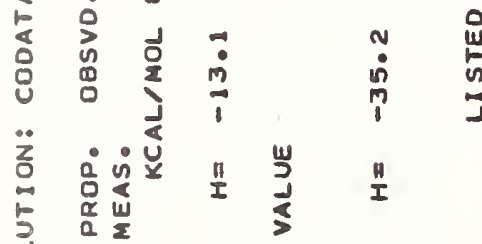

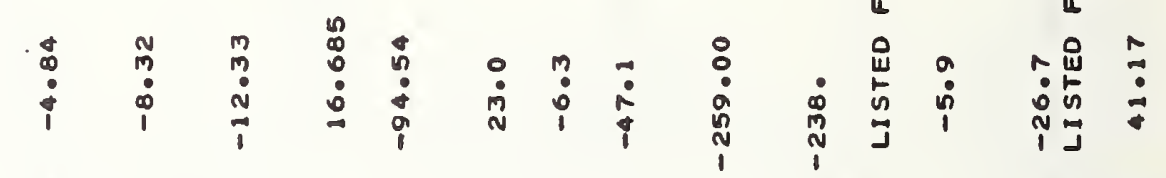

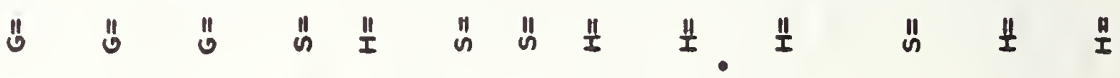<smiles>C1CCC[As]CCOCC1</smiles> 


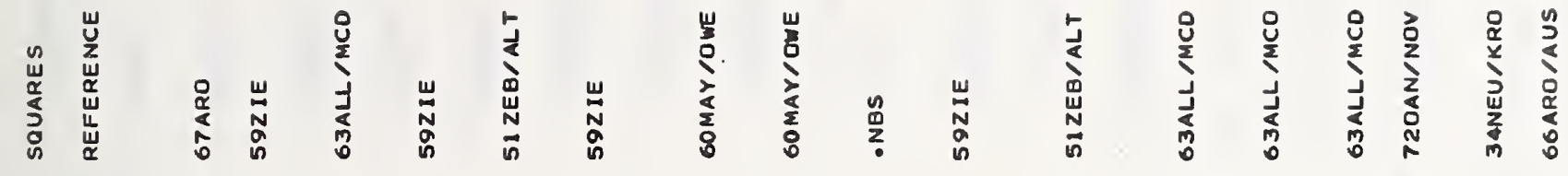

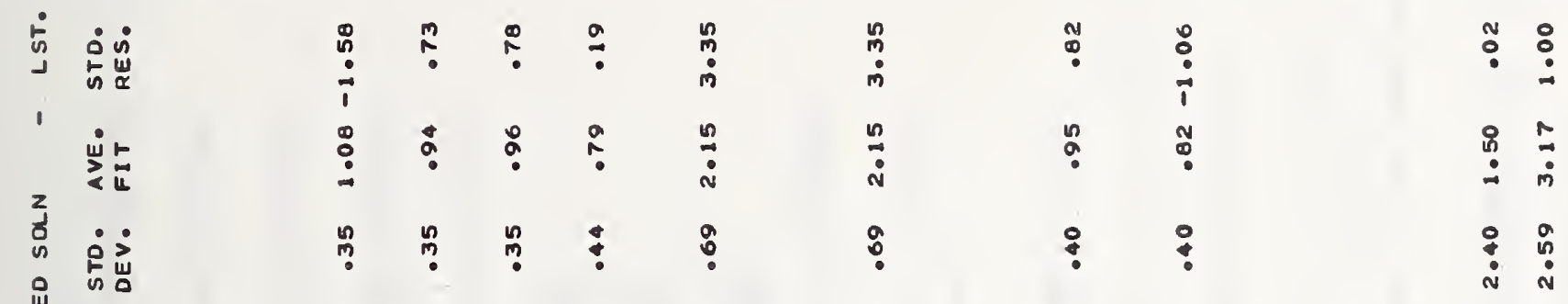

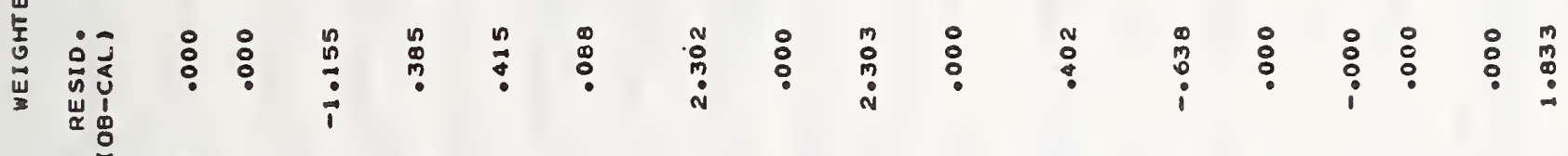

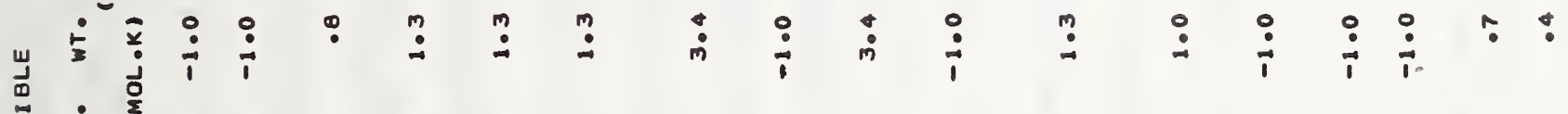

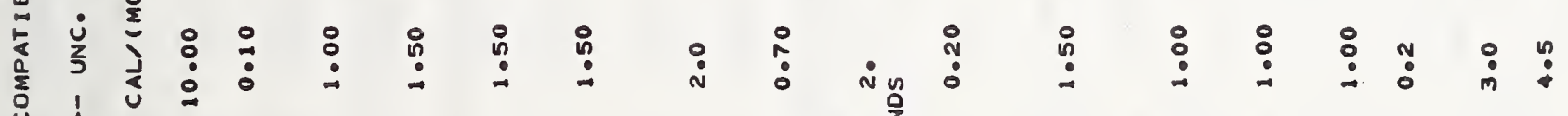

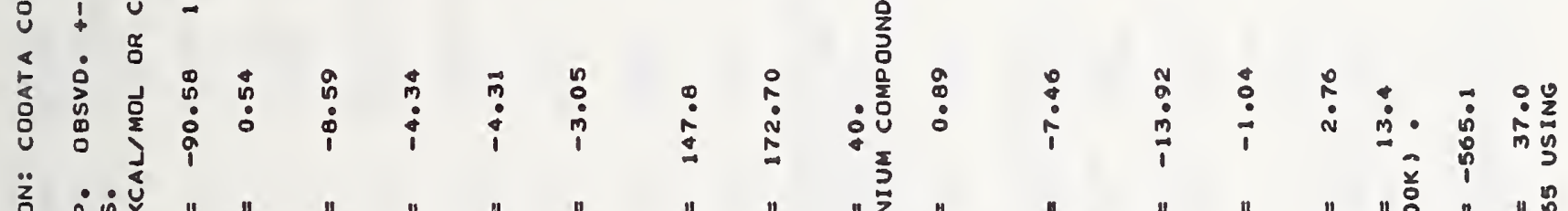

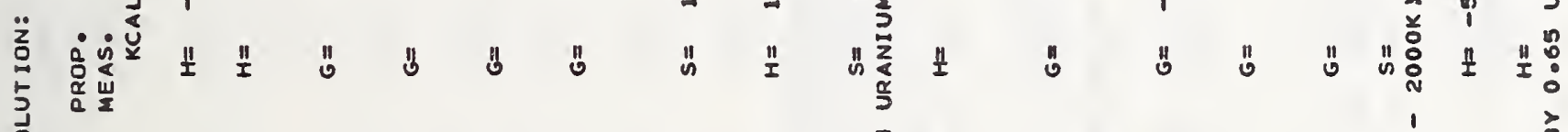
s. ह

(1) (1)

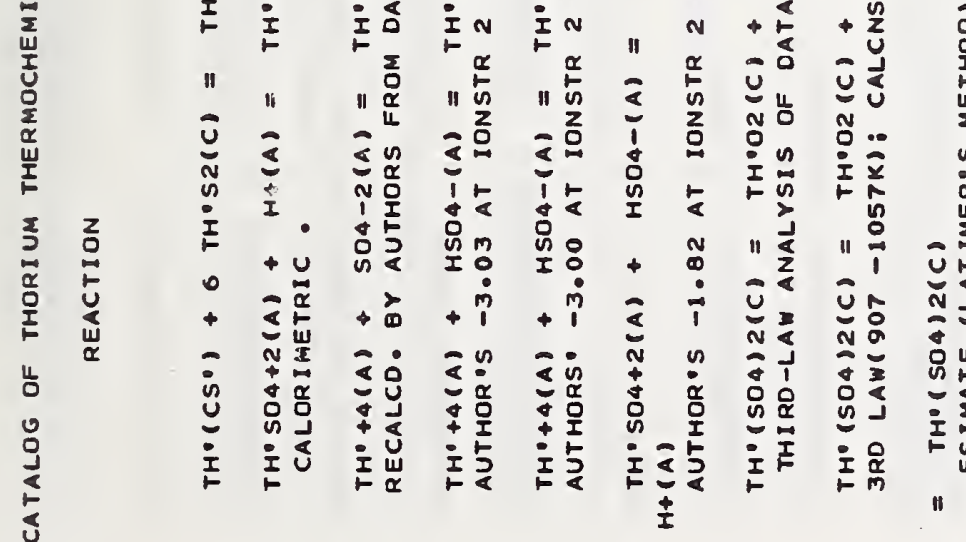

క

(1)

然

ì

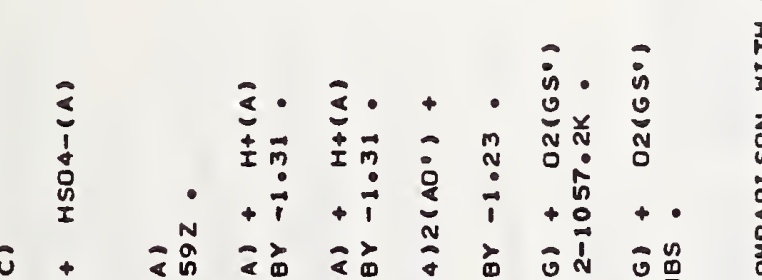

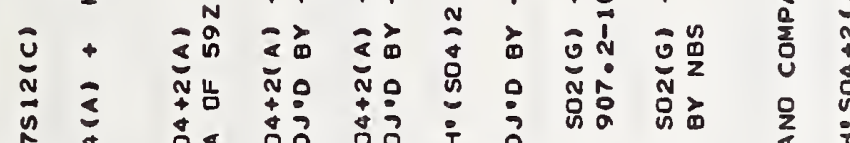

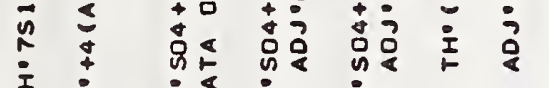

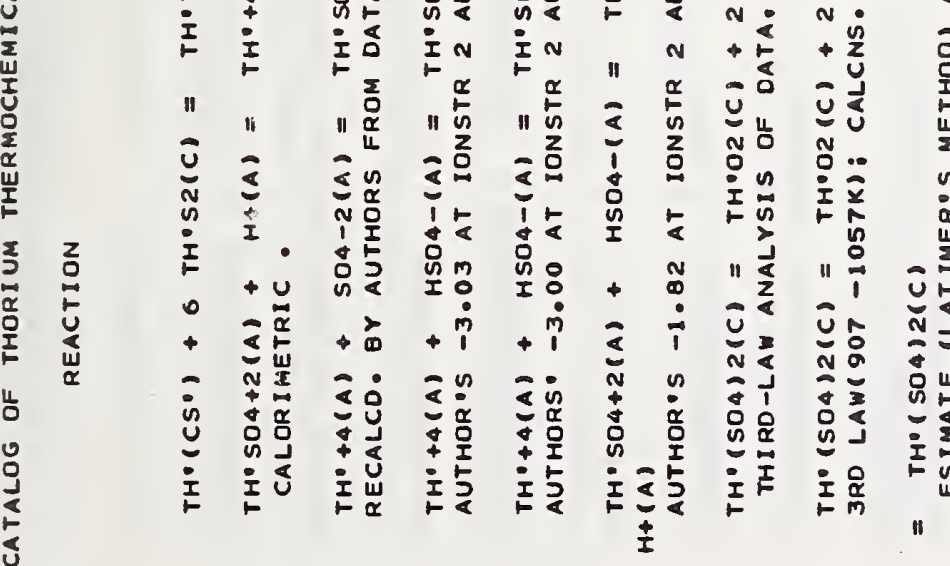

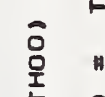

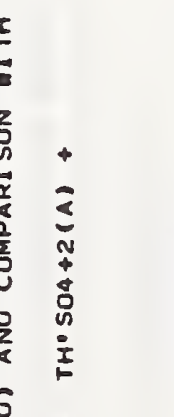

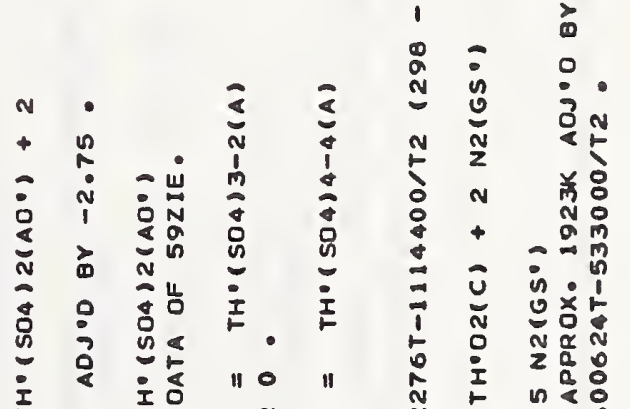

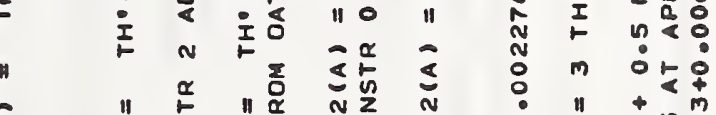

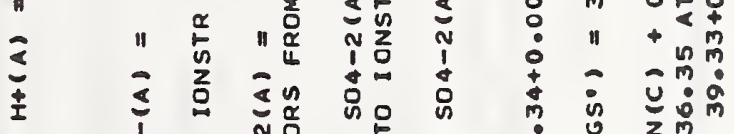

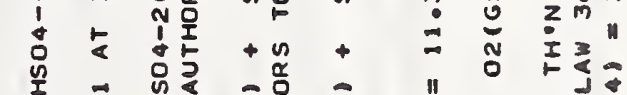




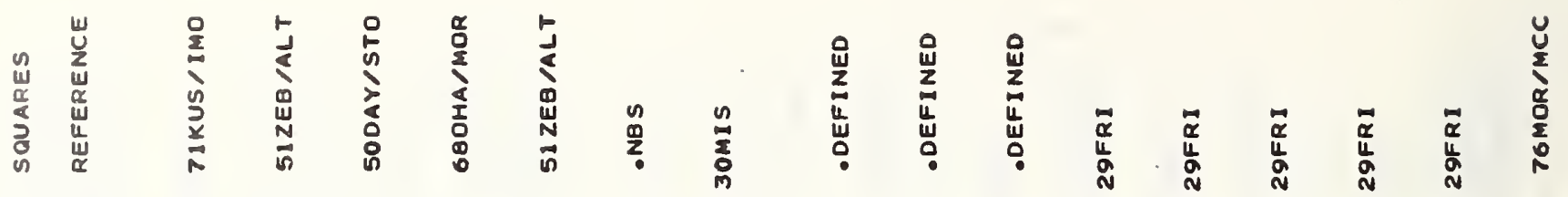

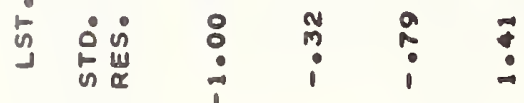

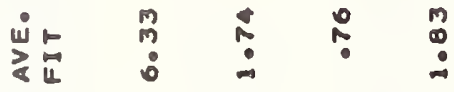

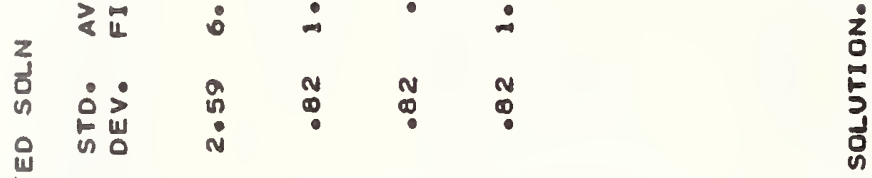

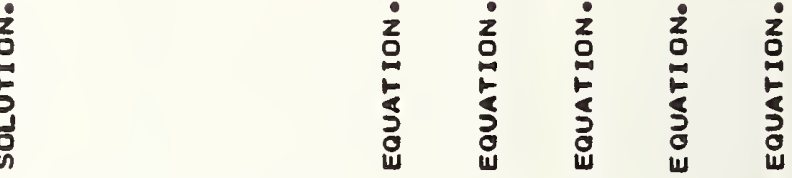

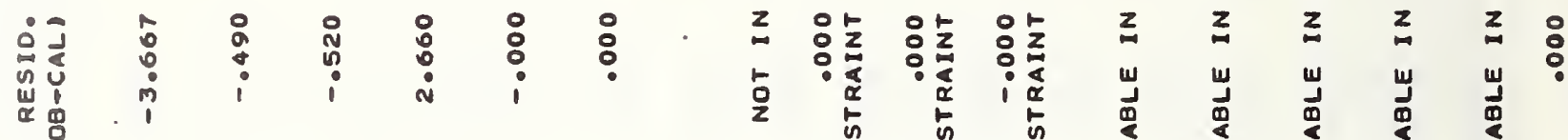

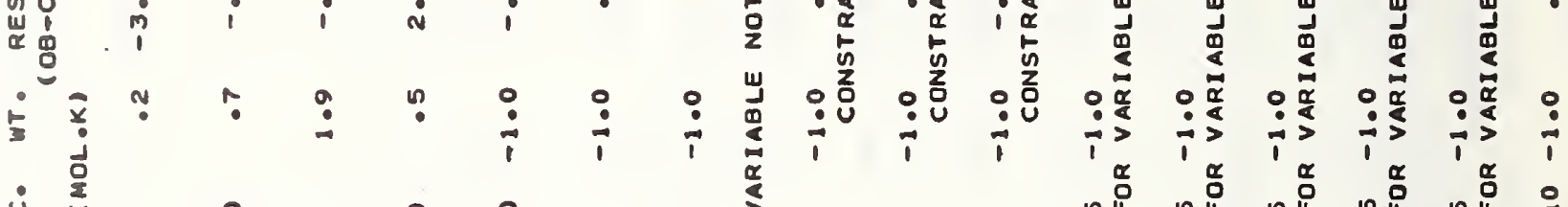

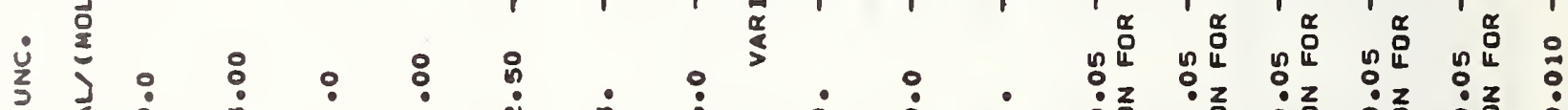

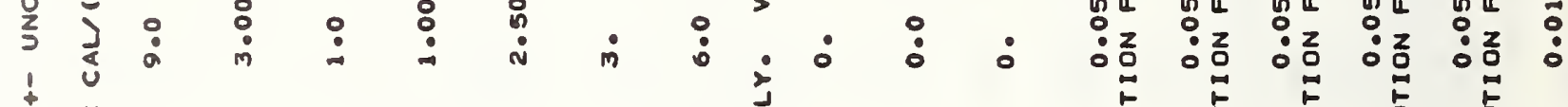

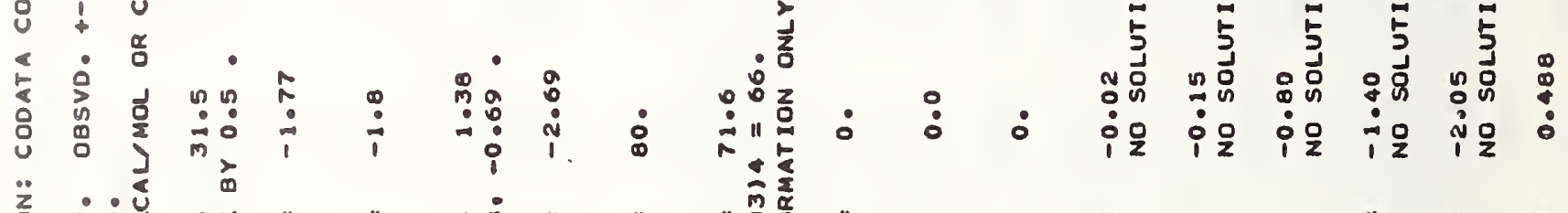

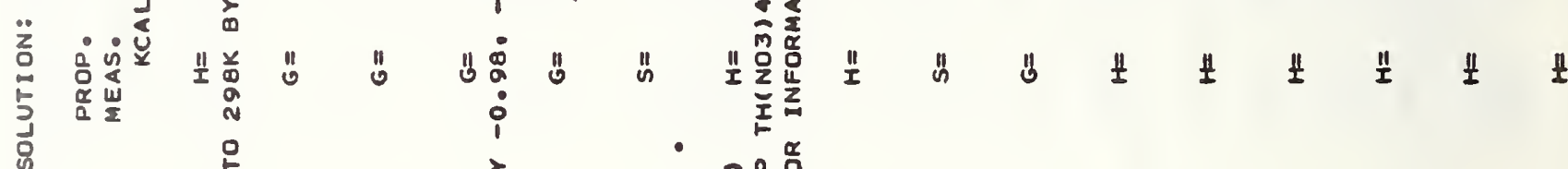

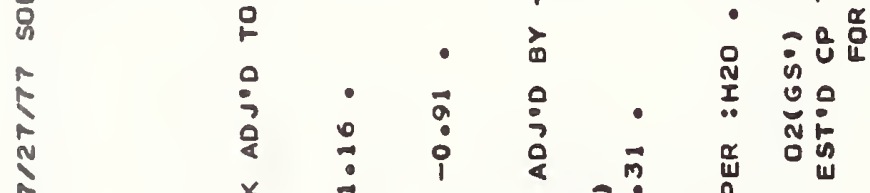

is

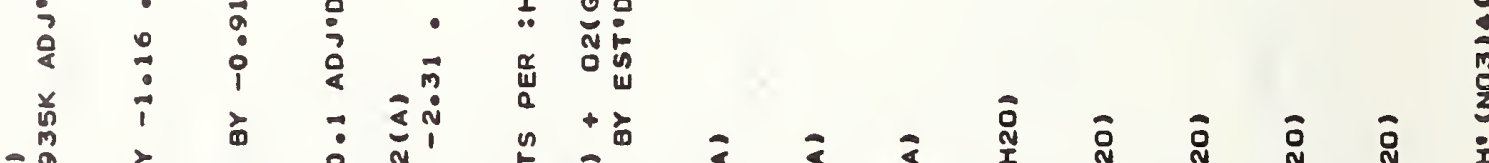

$\frac{1}{3}$

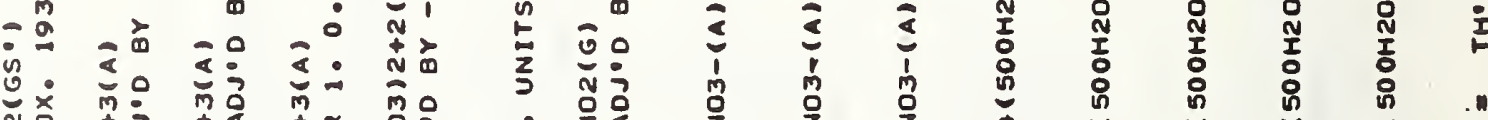

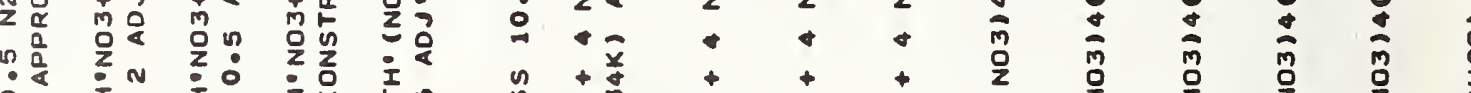

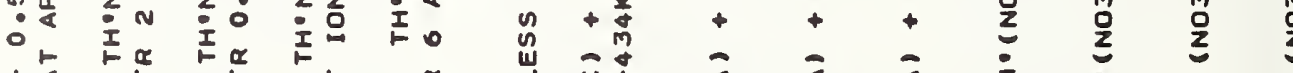

$+\frac{d}{0}$ "

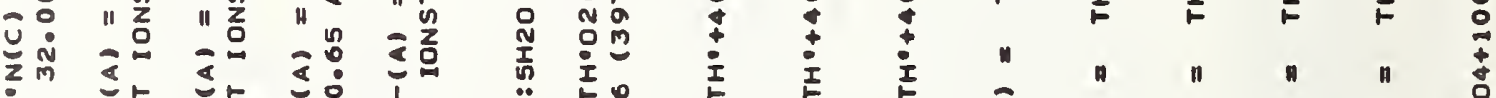

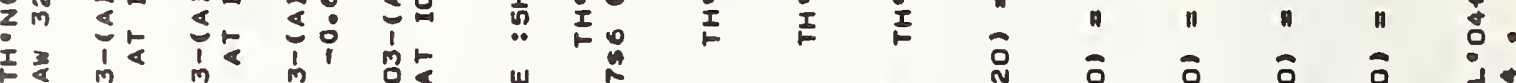

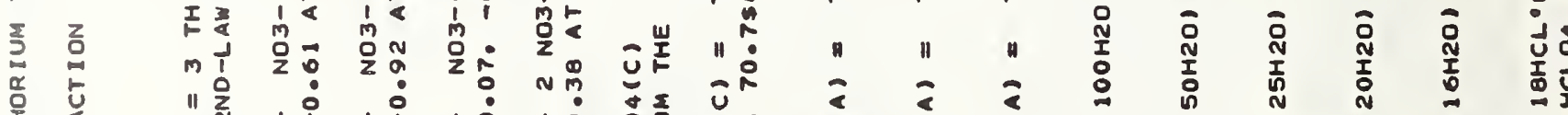

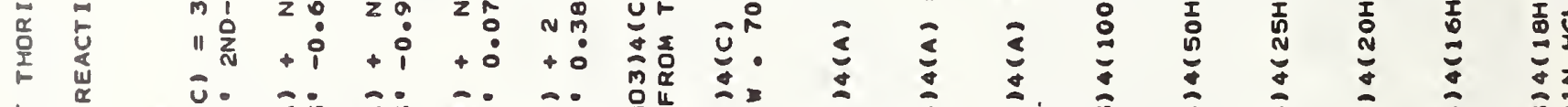

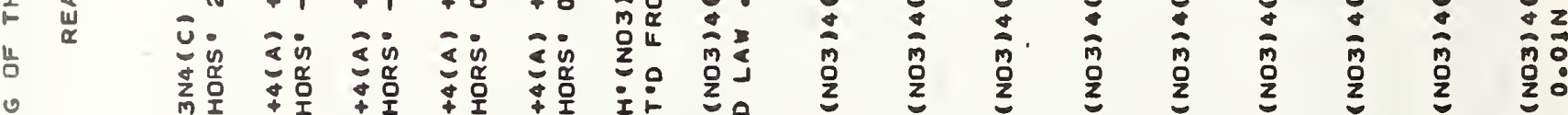

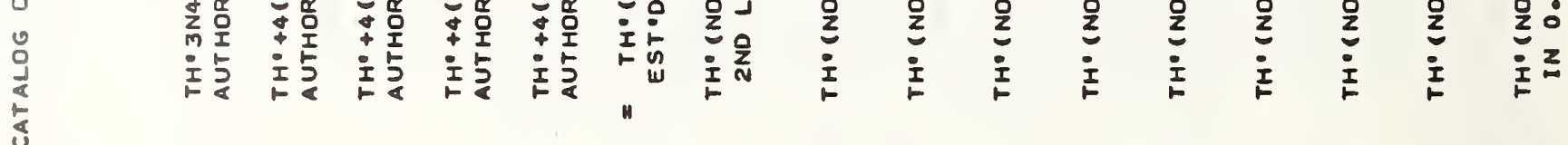

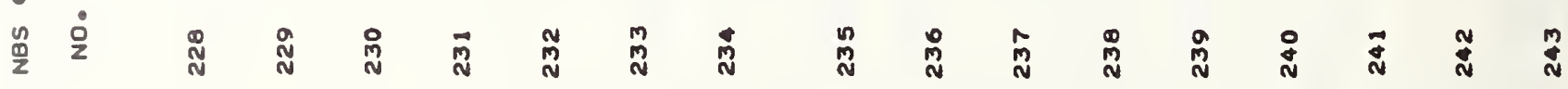




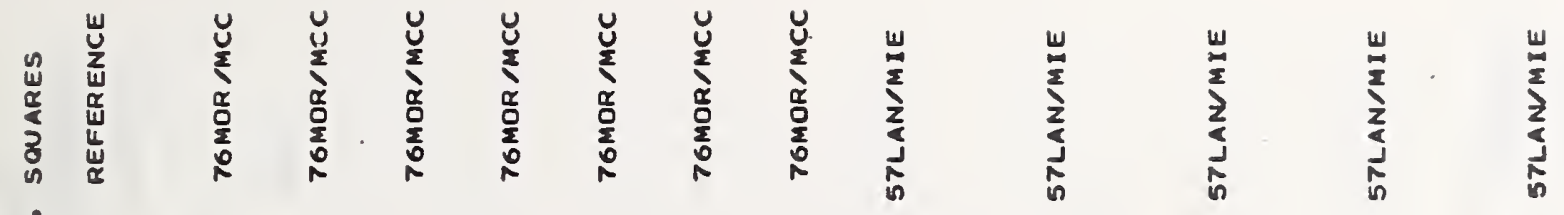

亨㪣

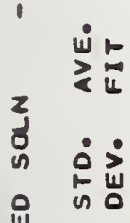

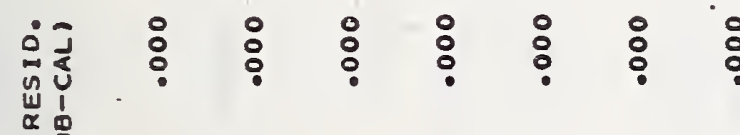

(

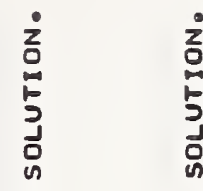

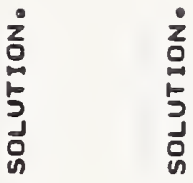

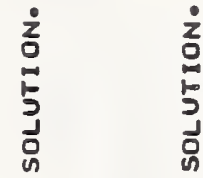

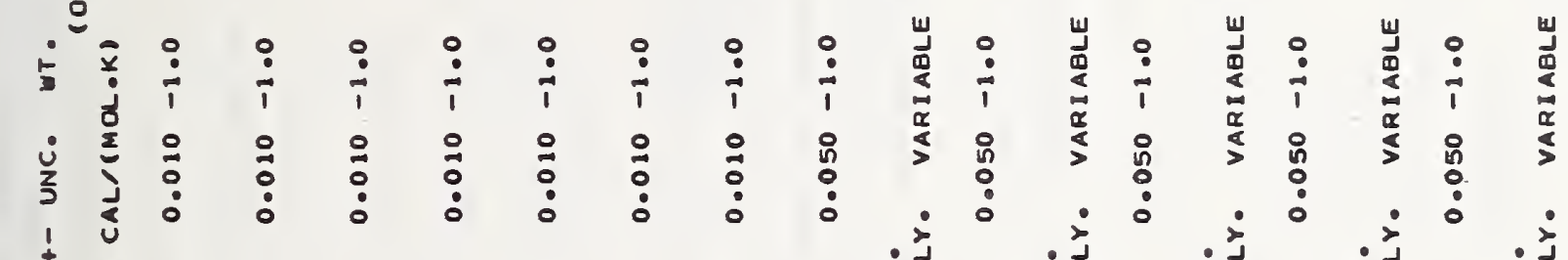

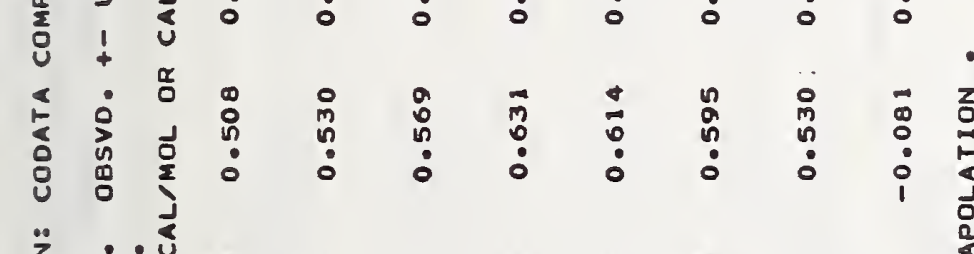

䇏竞

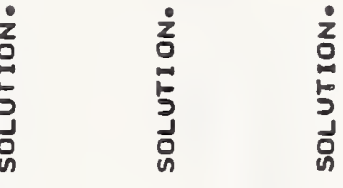

$z \quad z \quad z \quad z$

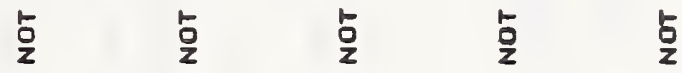

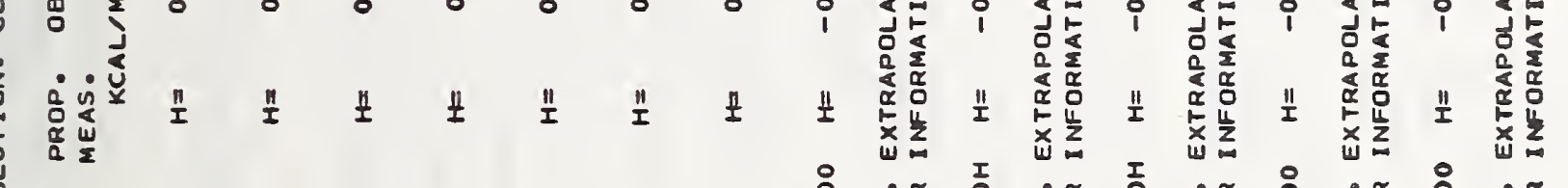

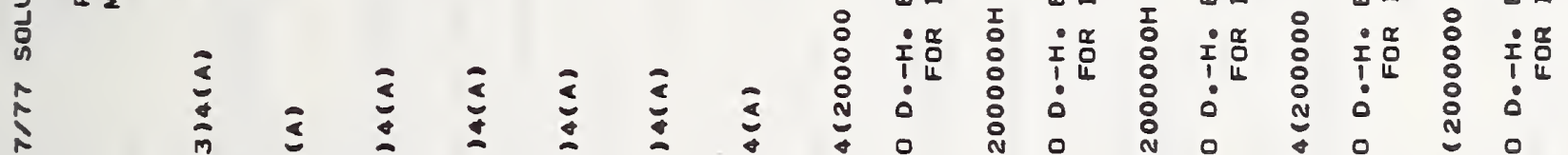

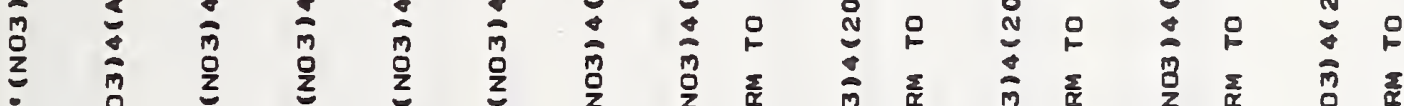

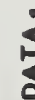

s

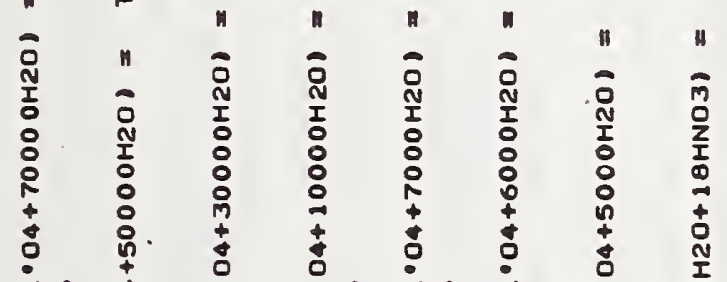

(1)

范

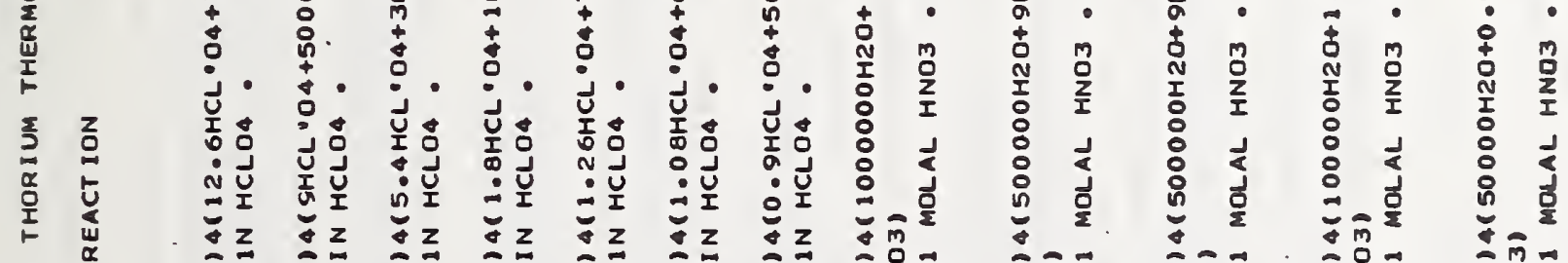

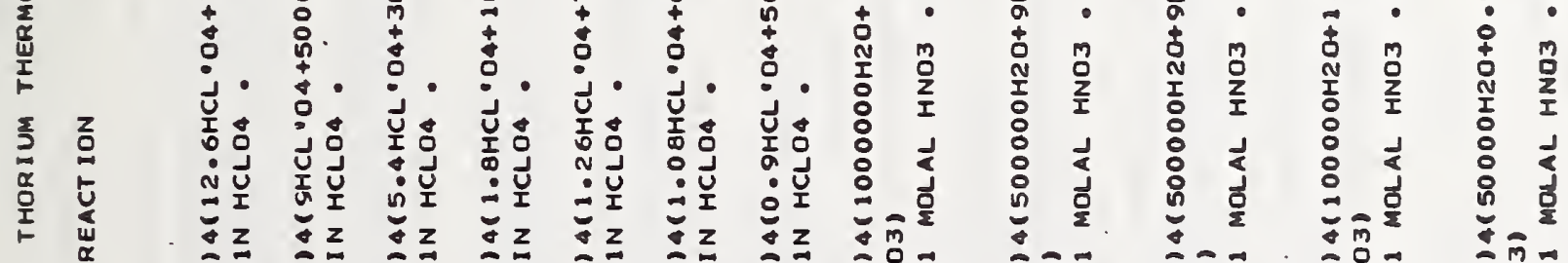

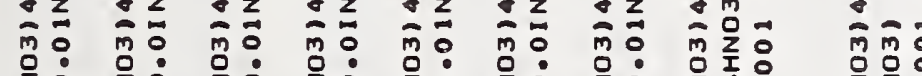

m:

:

Iz

.

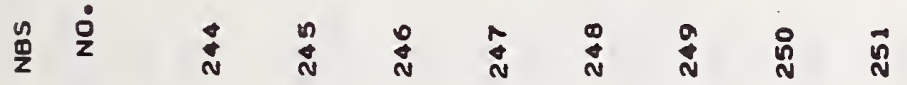

I

I

占 1 点"

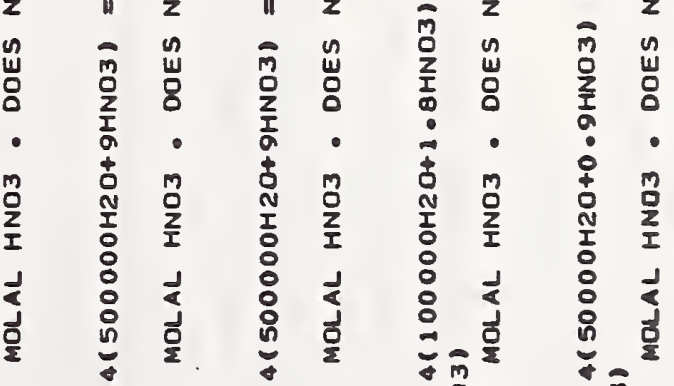

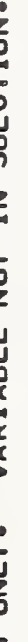




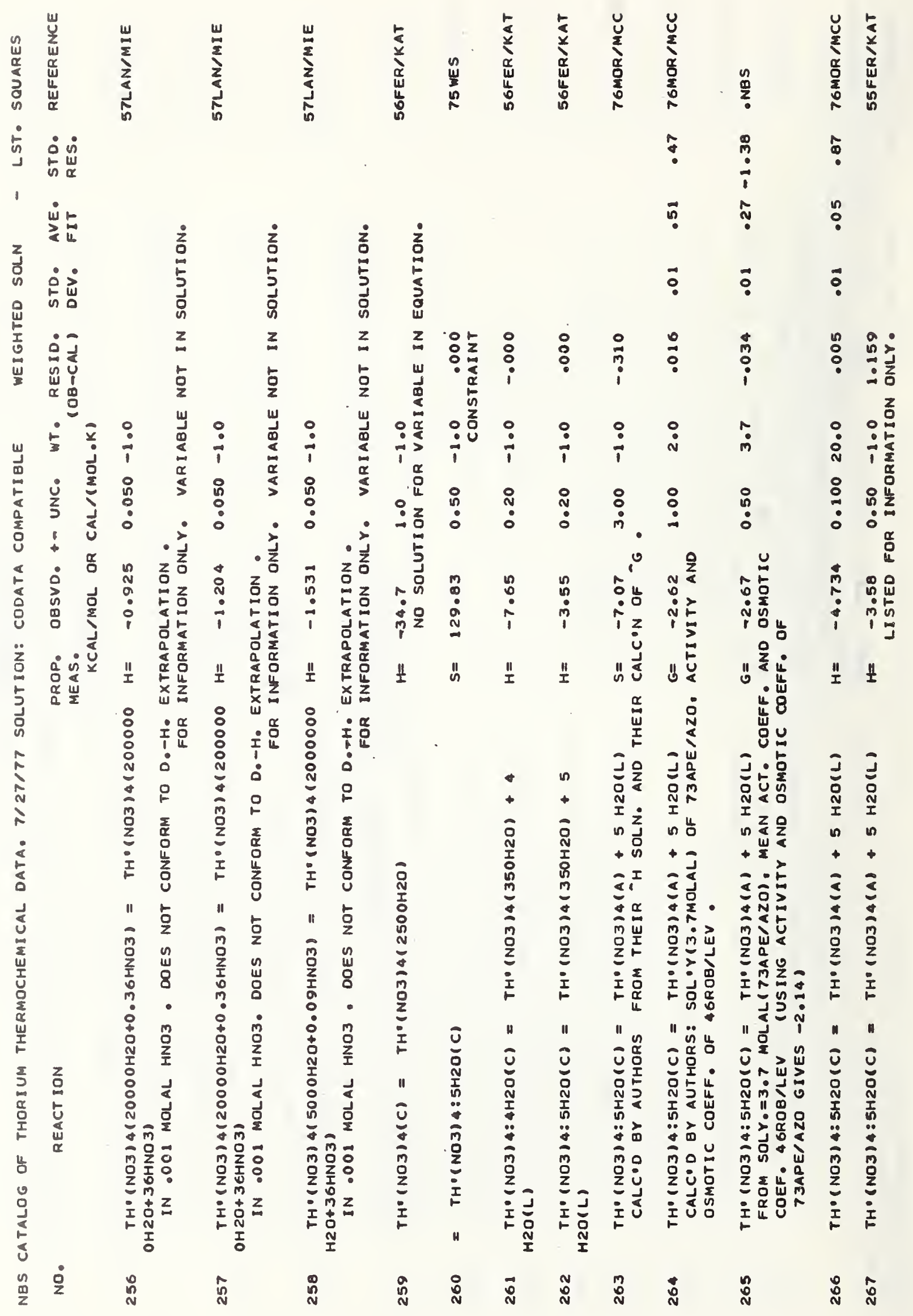




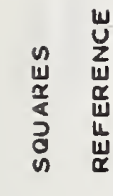

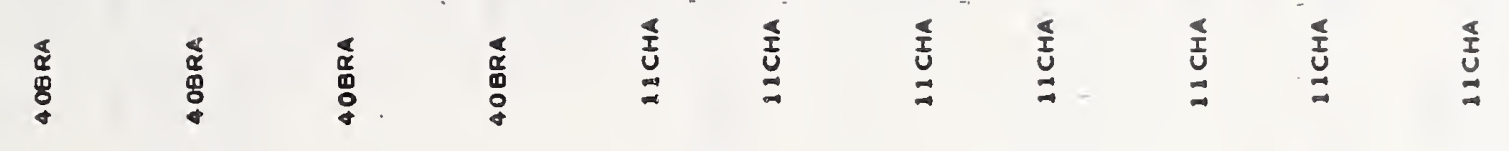

富高兽

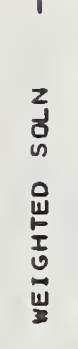

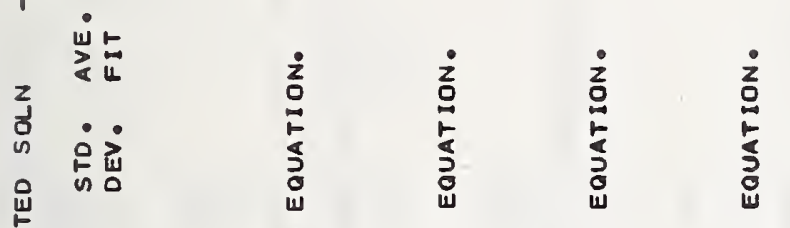

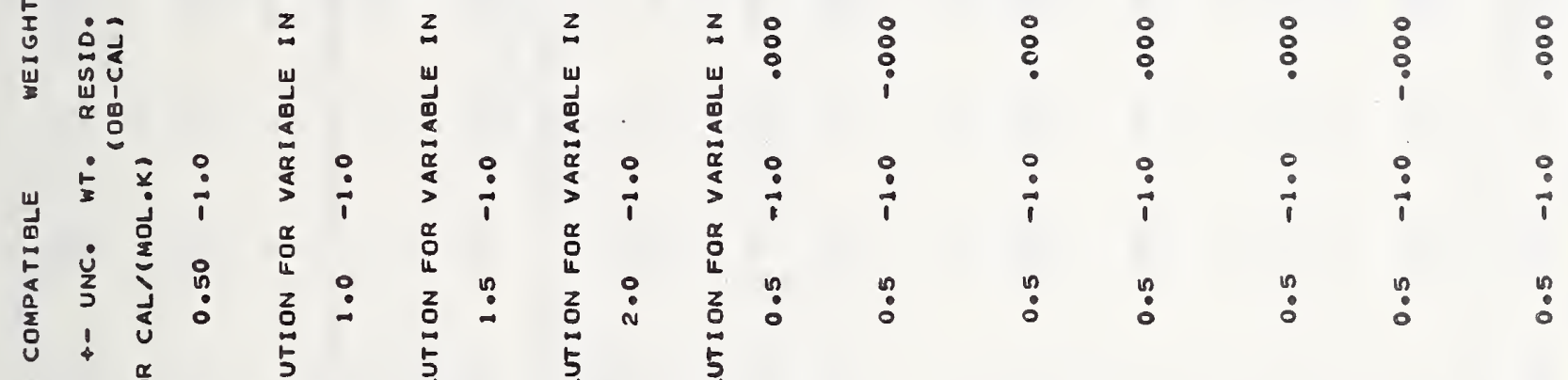

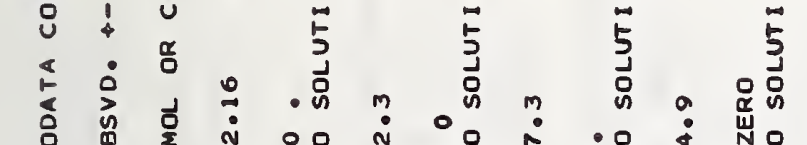

(1)

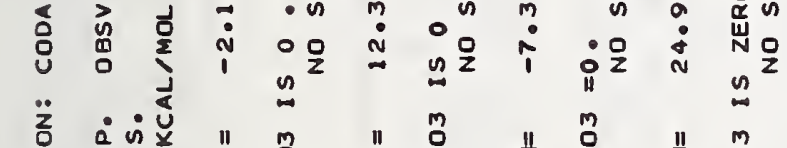

$\cos ^{2}$

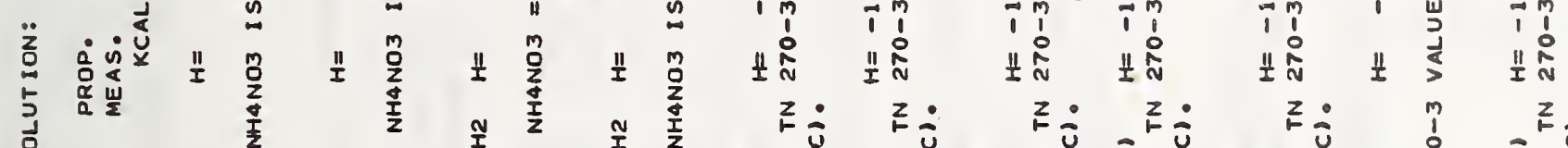

ริ

is

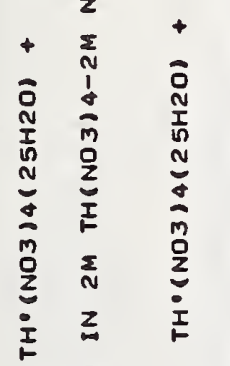

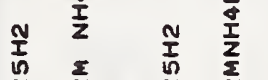

ż: $\quad z \dot{v}$

$z: \quad z \dot{i}$

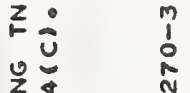

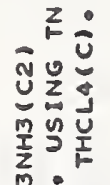

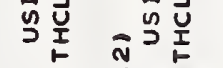

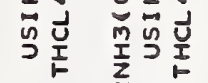

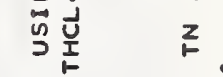

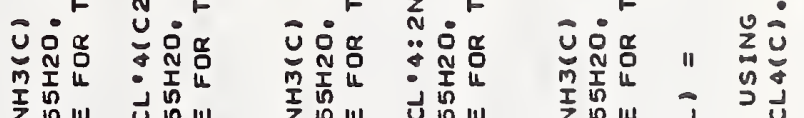

要品

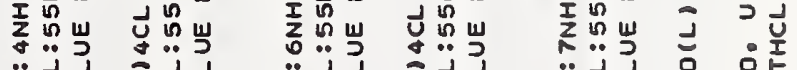

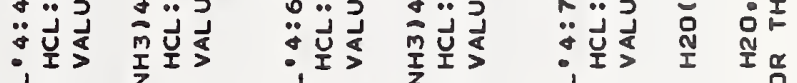

- T

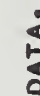

I I I

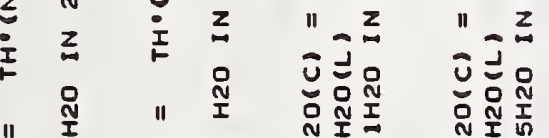

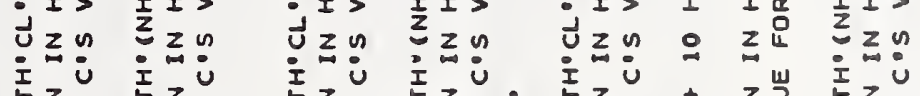

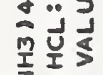

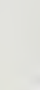

(1)

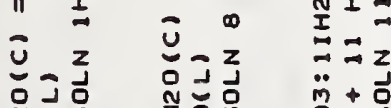

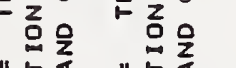

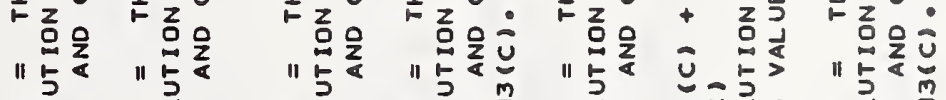

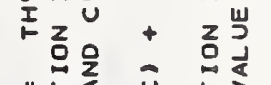

I $z_{0}^{0}$

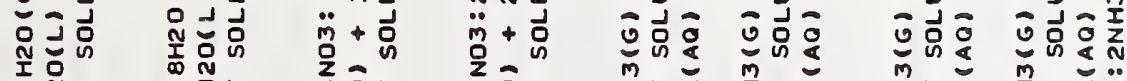

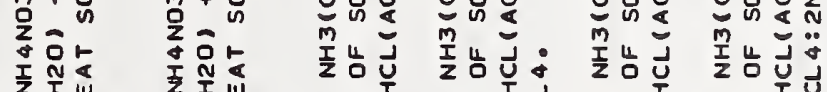

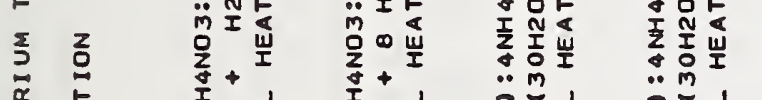

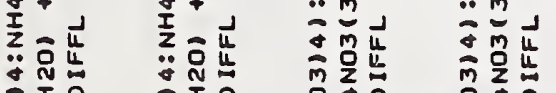

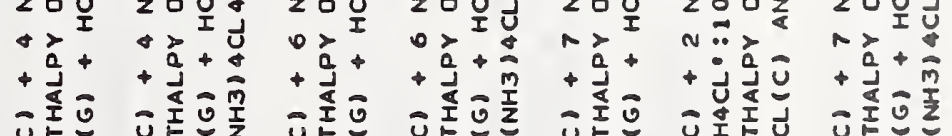

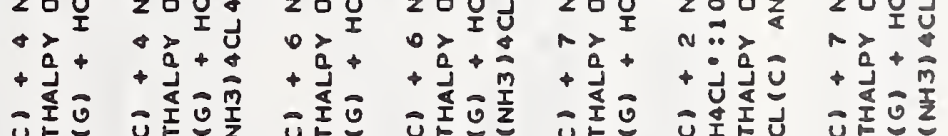

它品品

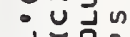

要震

mu

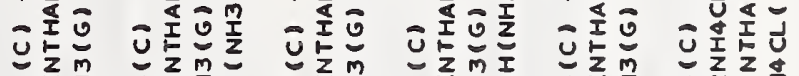

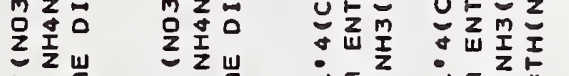

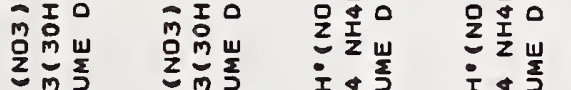

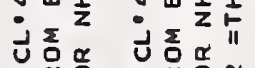

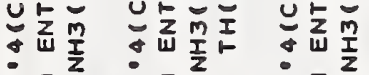

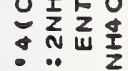

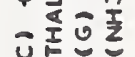

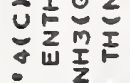

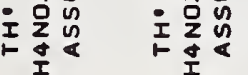

2
5

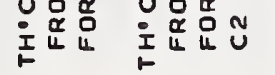

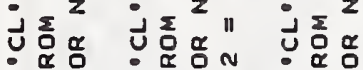

נ艹

$\dot{0} \Sigma^{2}$

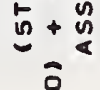

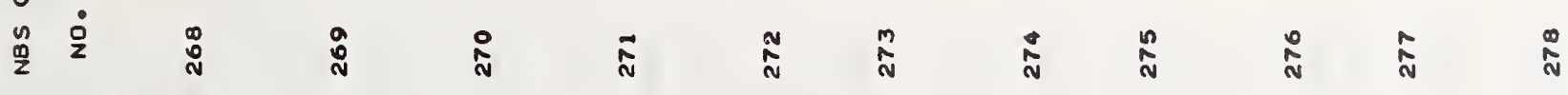

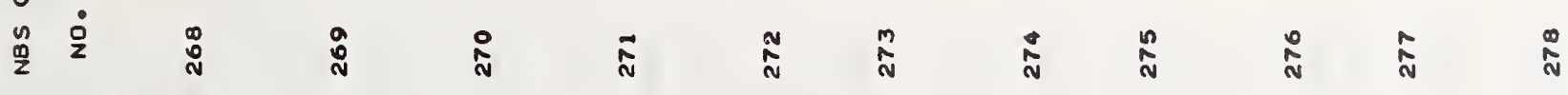

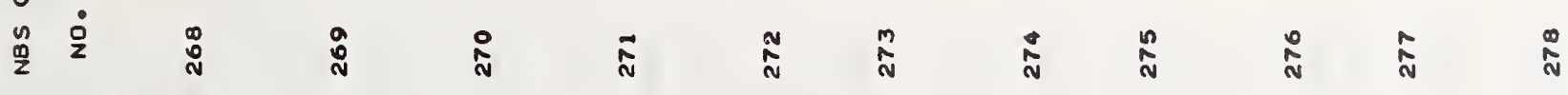

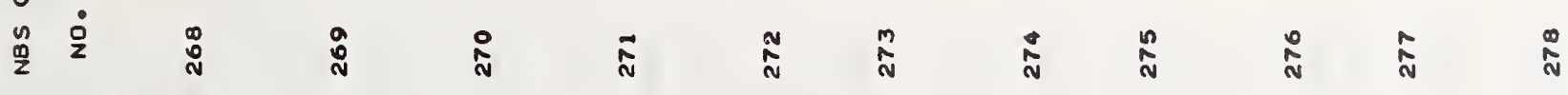

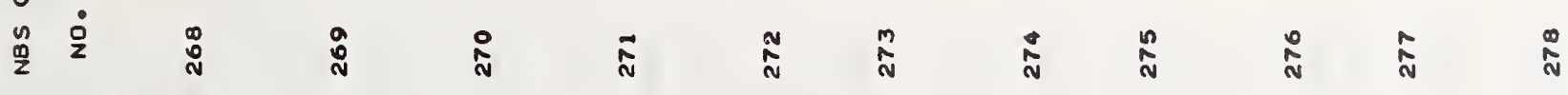

I

主花罚 


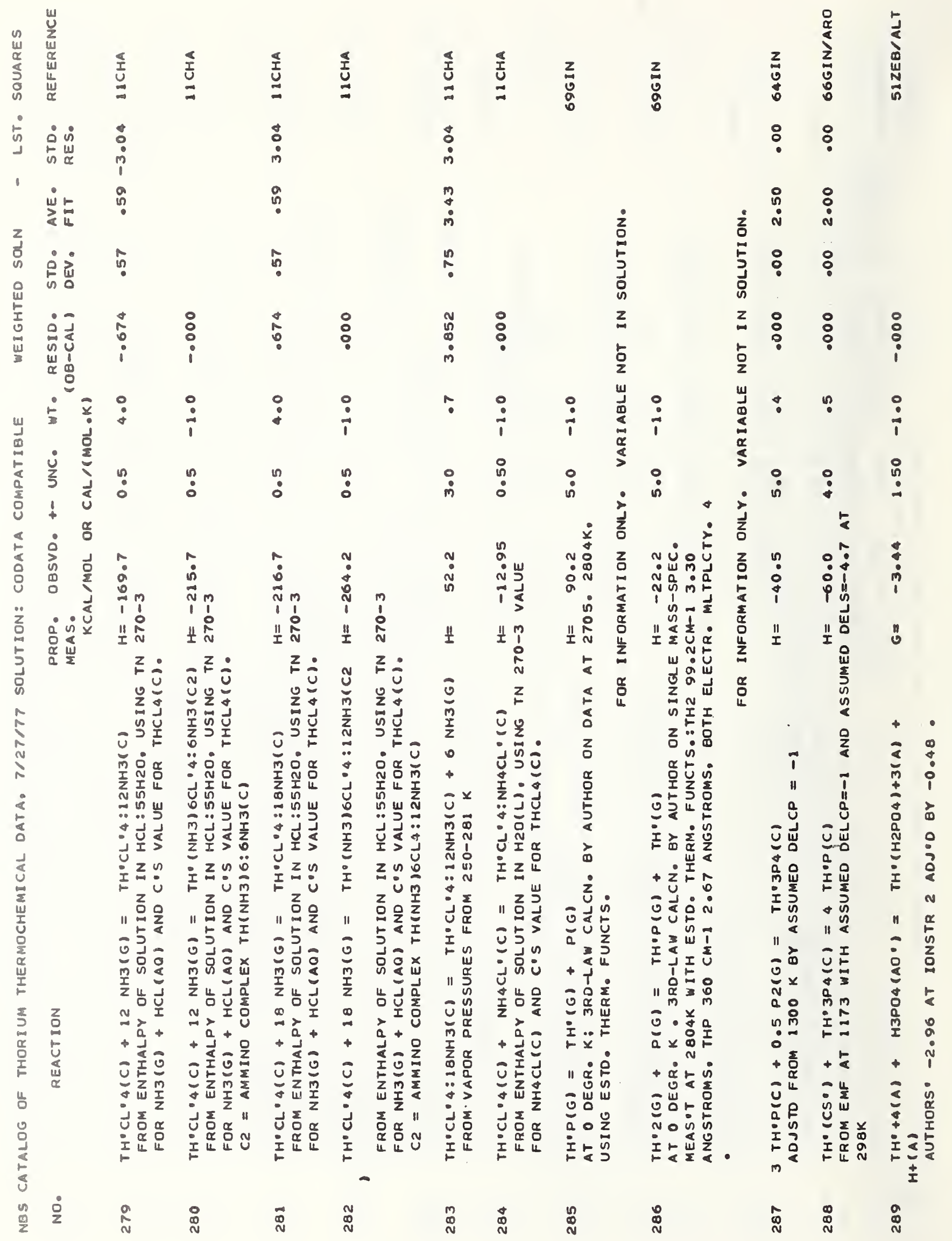




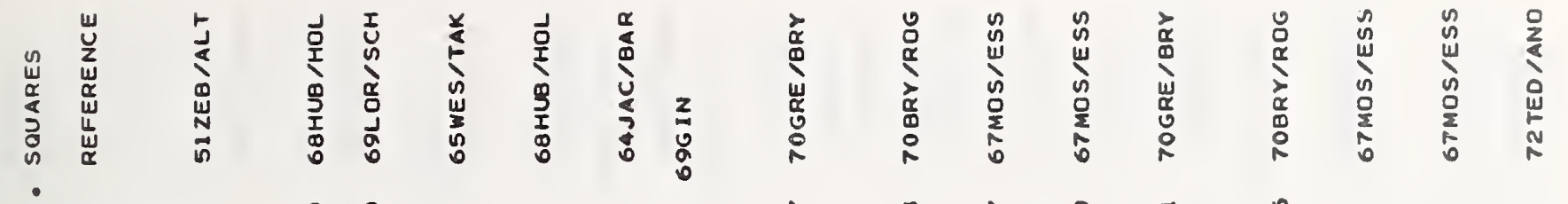

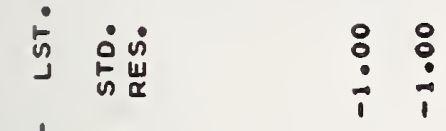

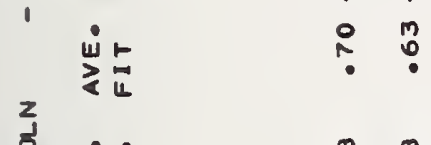

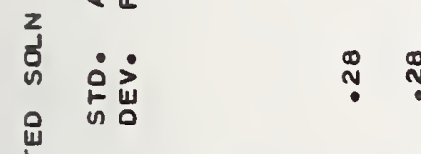

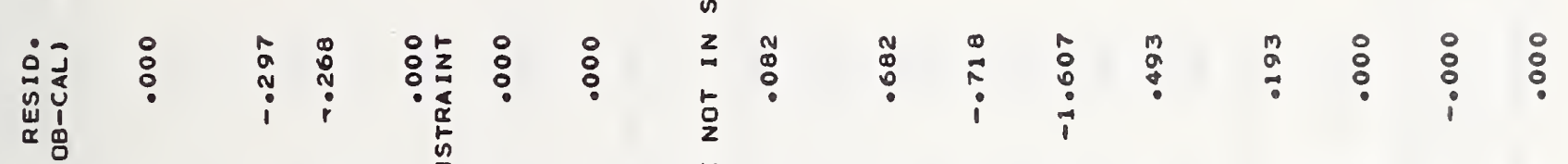

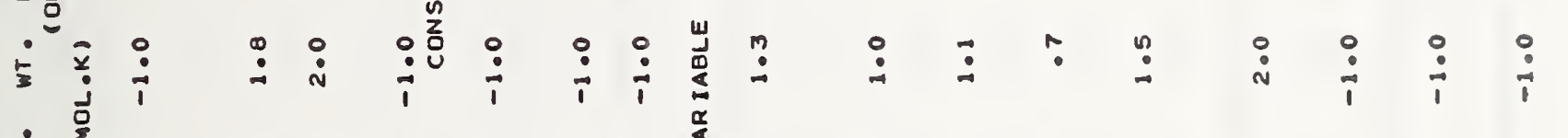

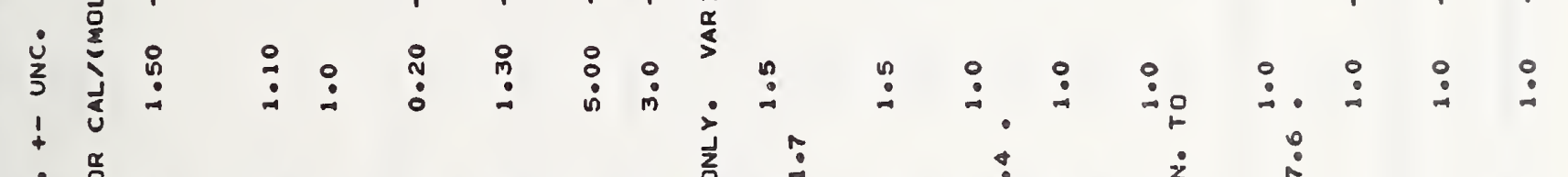

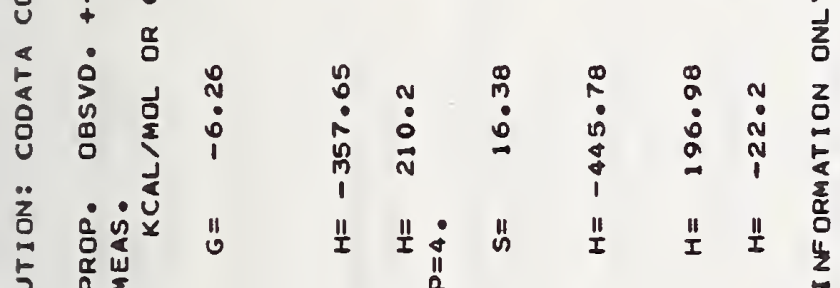

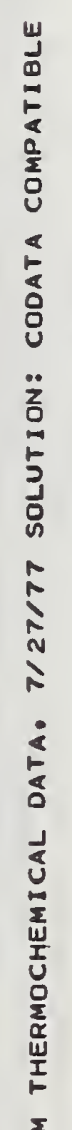

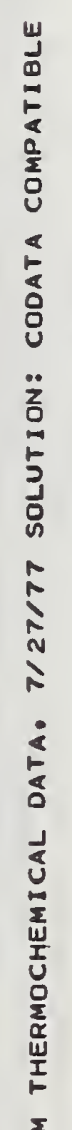

JuJ

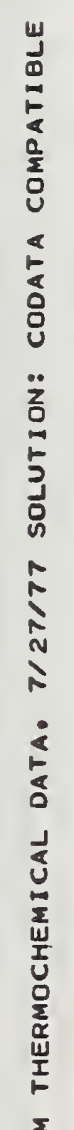

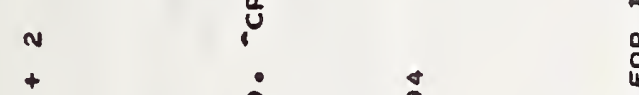<smiles>C#[W]</smiles>

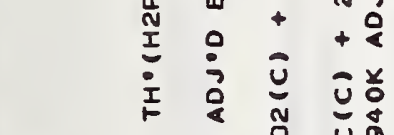

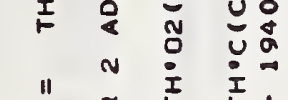

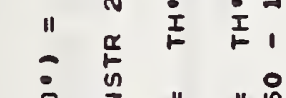

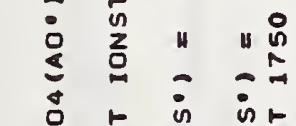

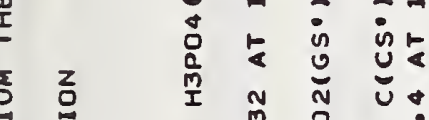

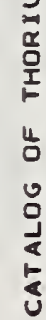

(1)

唡

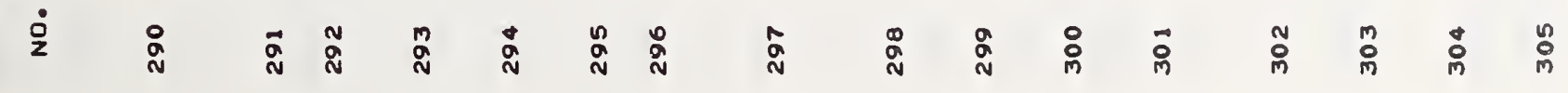




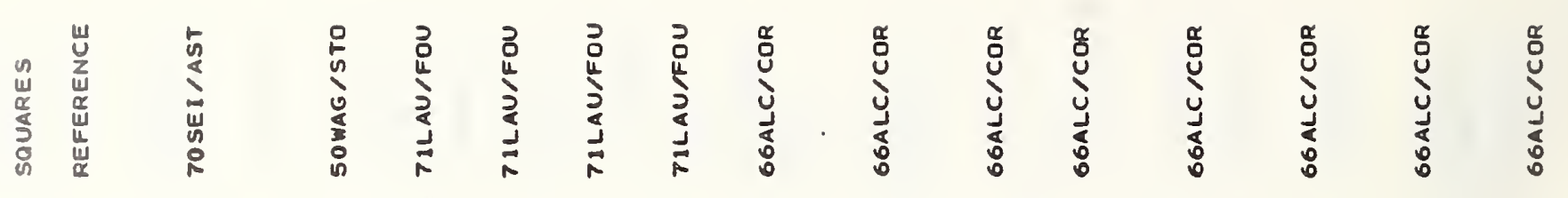

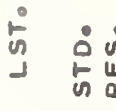

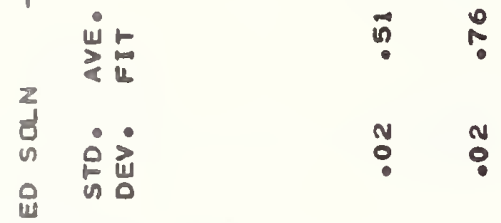

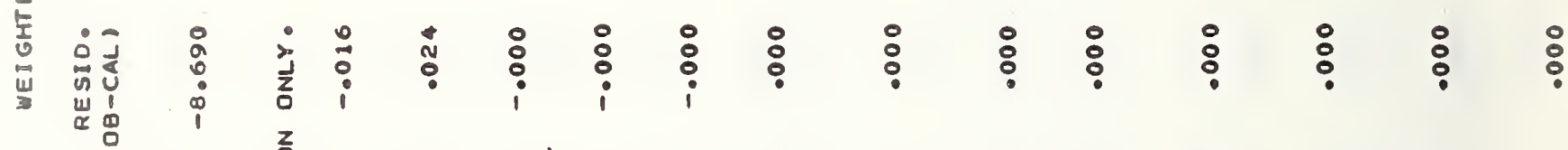

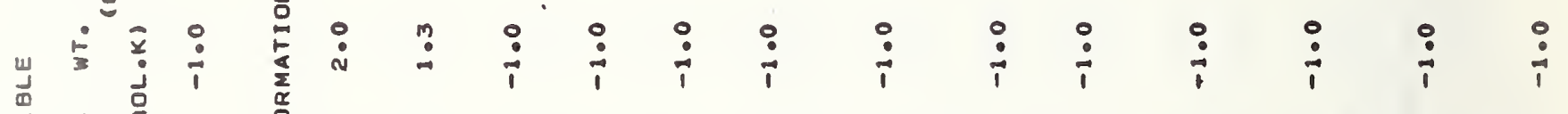

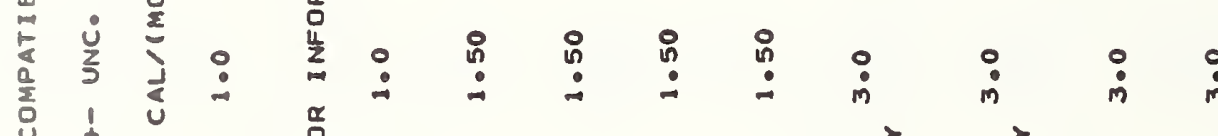

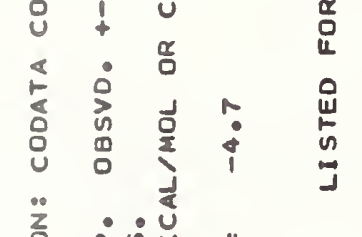

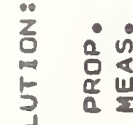

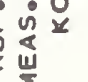
is

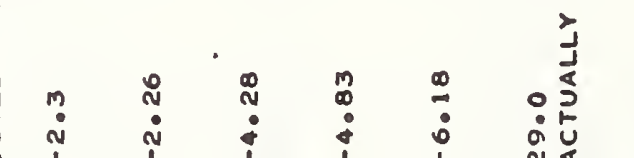

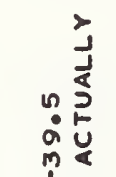
- is $\frac{x^{2}}{3}$

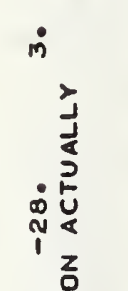

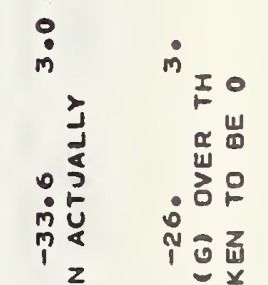
" 范 至苫

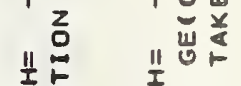
$\sum_{\substack{n\\}}^{n}$

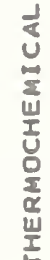

章 \& n 0 a n 2 a

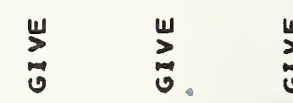

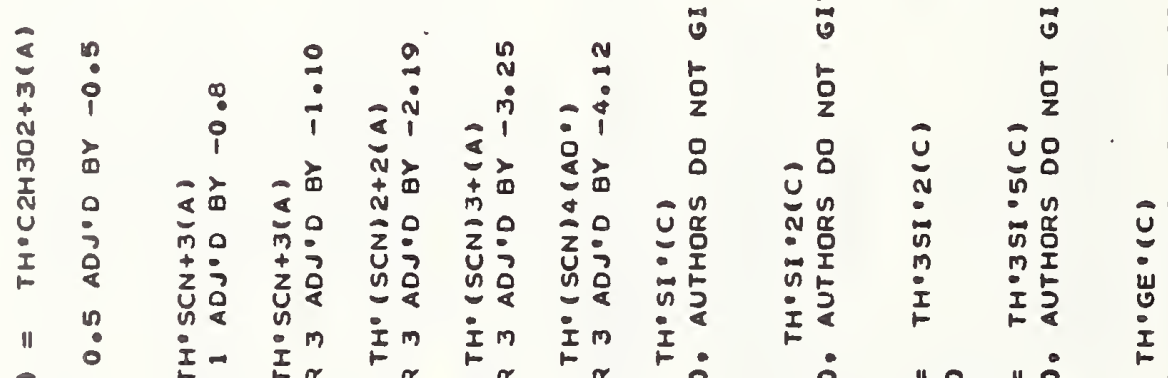

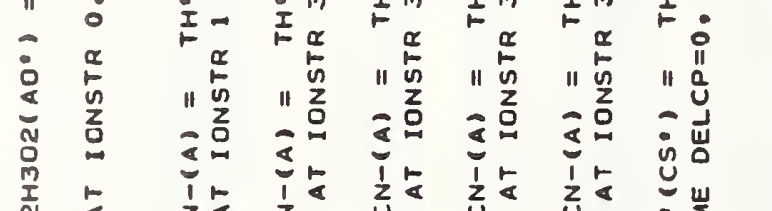

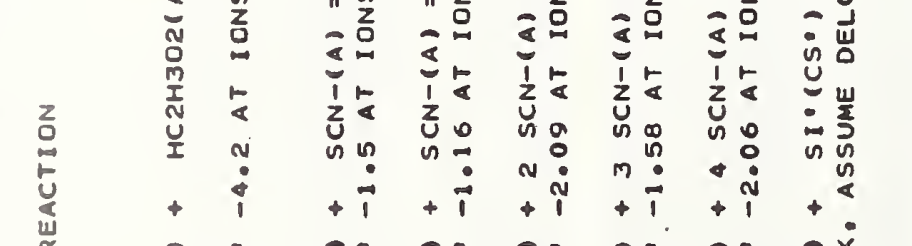

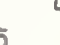

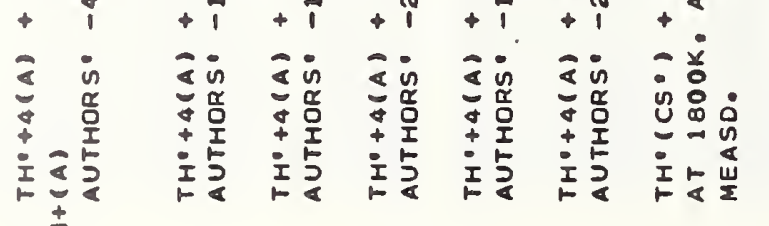

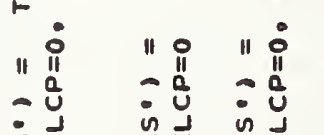

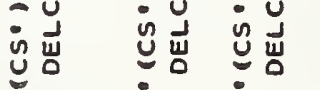
U : I 占告 是定 $\circ$ 总兽 $\approx$

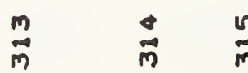
$\frac{n+2}{n+2}$ $\stackrel{\circ}{\circ} \div$ 


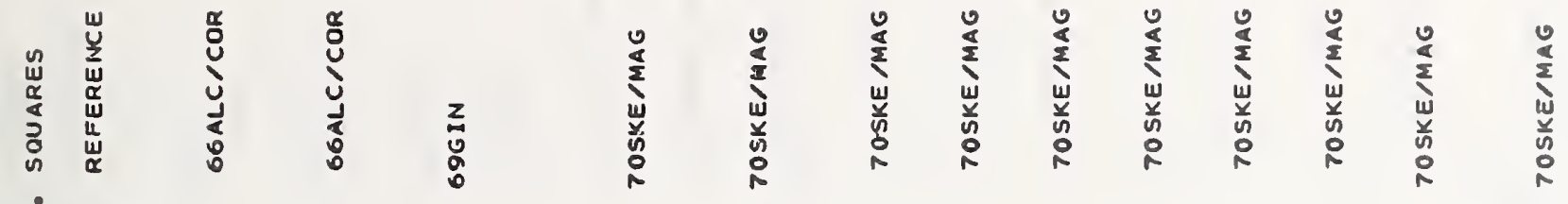

占定品

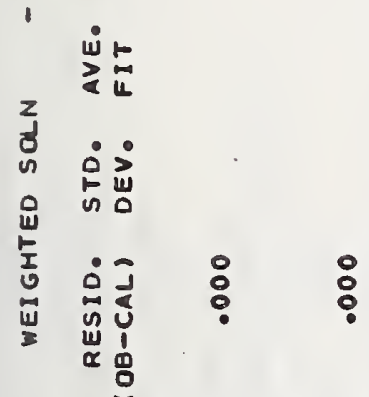

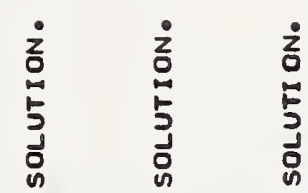

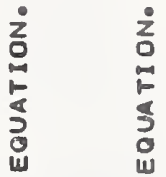

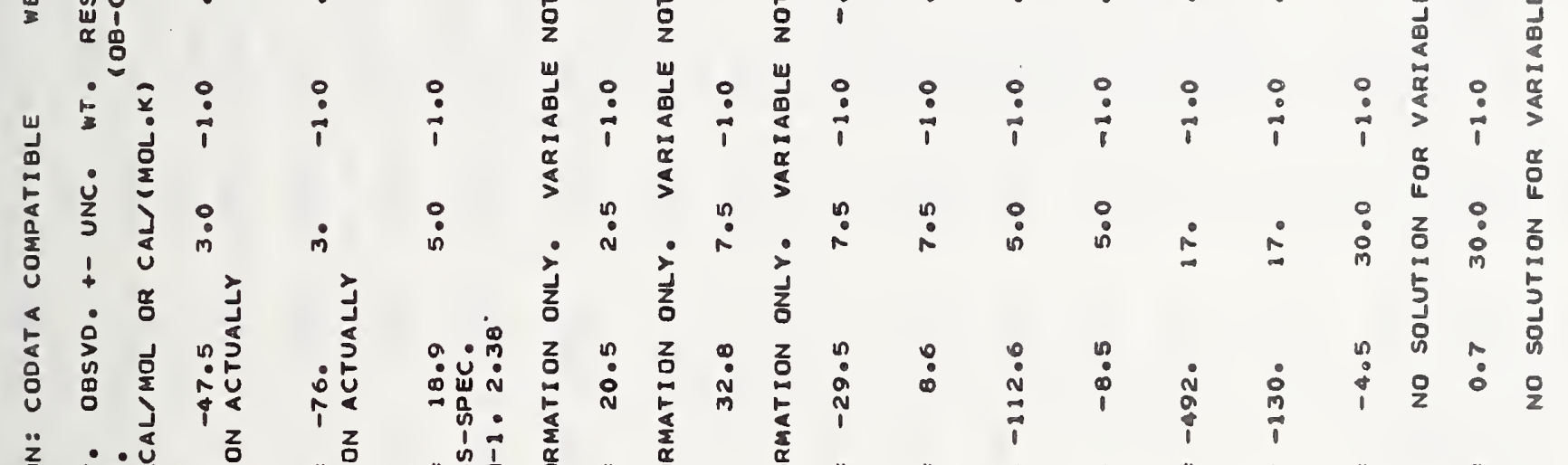

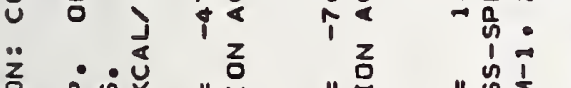

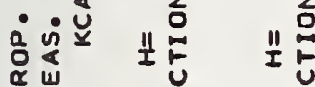

告

II

山्ग

$\sum_{0}^{w} \quad \sum_{0}^{\omega} \quad \sum_{i=1}^{\infty}$

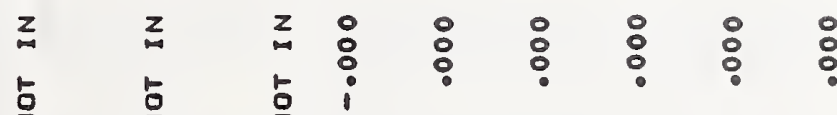

z:

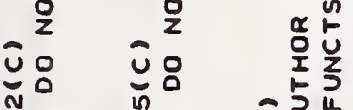

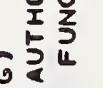

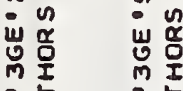

난

à站

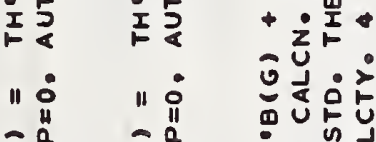

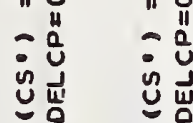

I $=$ แ

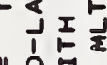

u山

岕㸚 岁

ơ

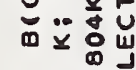

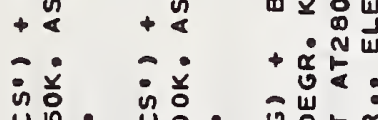

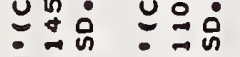

லै

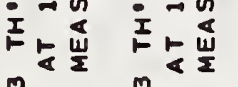

o 0 in

(1)

迹

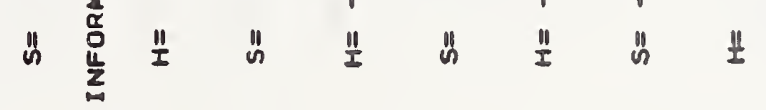

y.

แ。

:

选

哭

$\dot{0}$

in

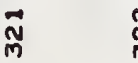

$\underset{N}{N}$

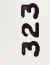

N

$\stackrel{n}{N}$

$\stackrel{8}{\circ}$

$\$$

:

\&

盟 兽

D 


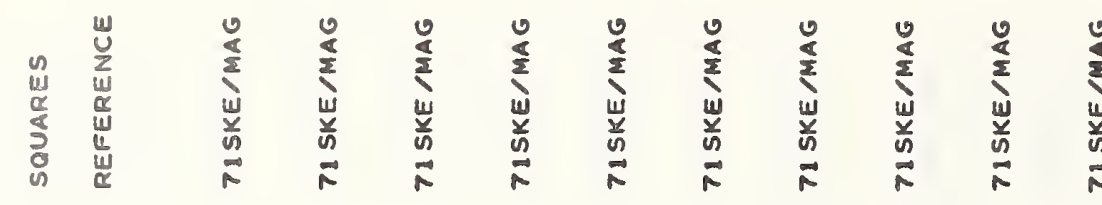

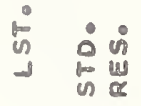

2

$\sum_{k}^{w}$

这

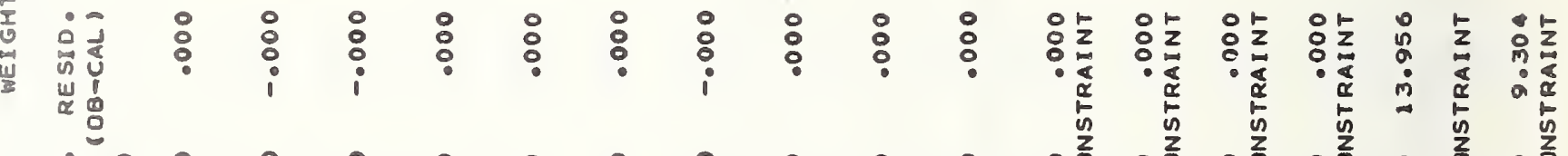

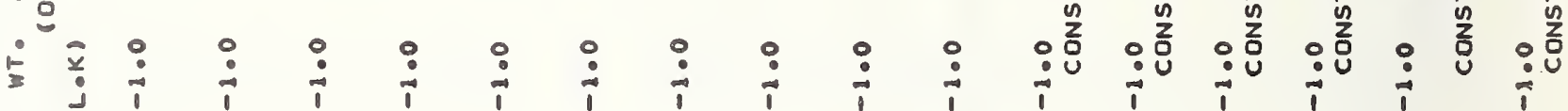

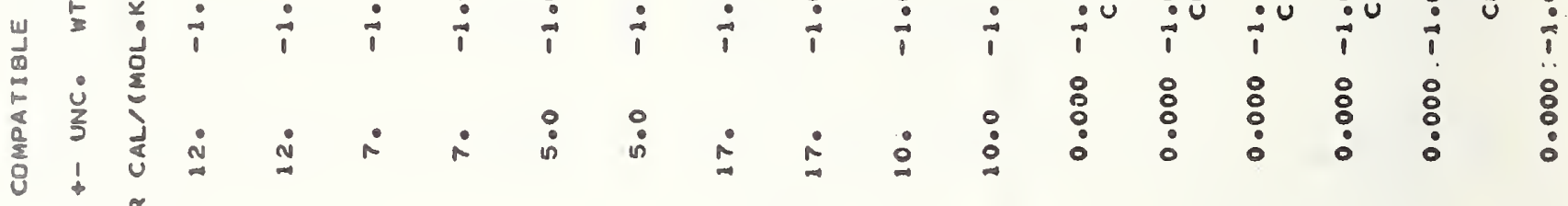

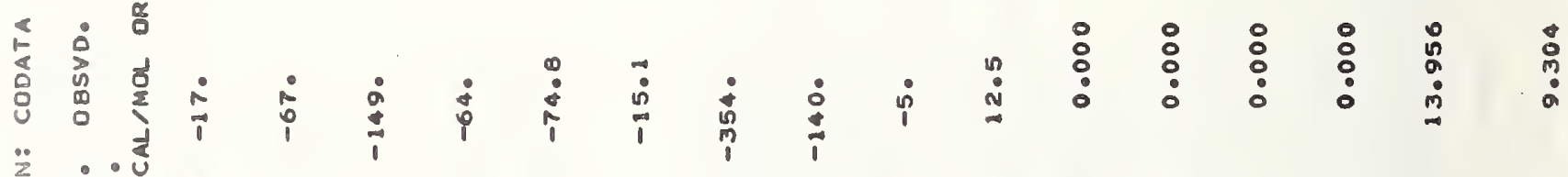

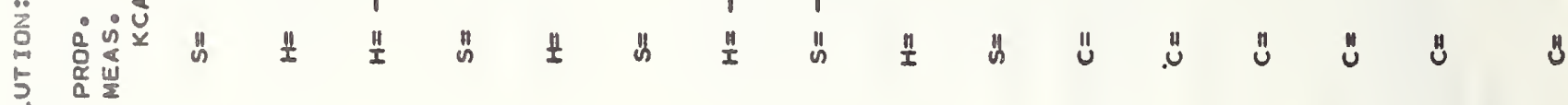

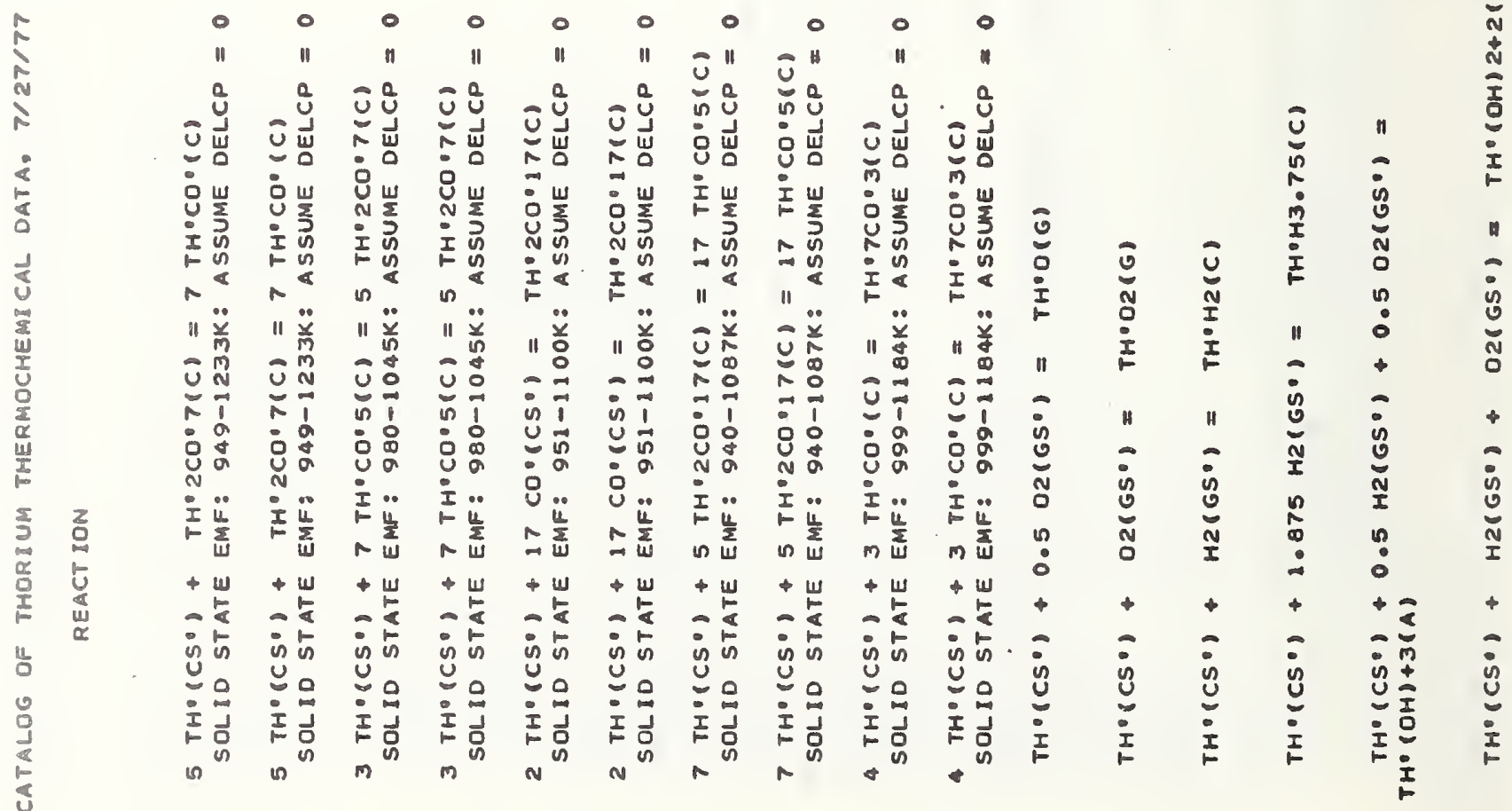

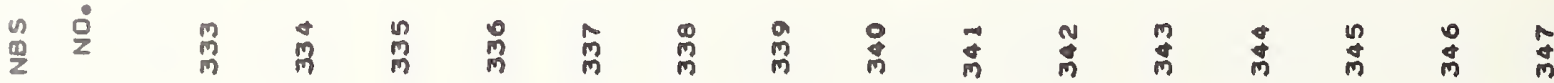




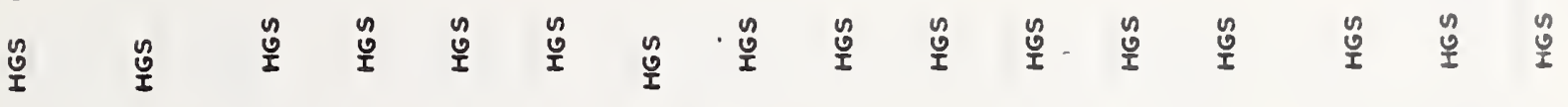

容蜜

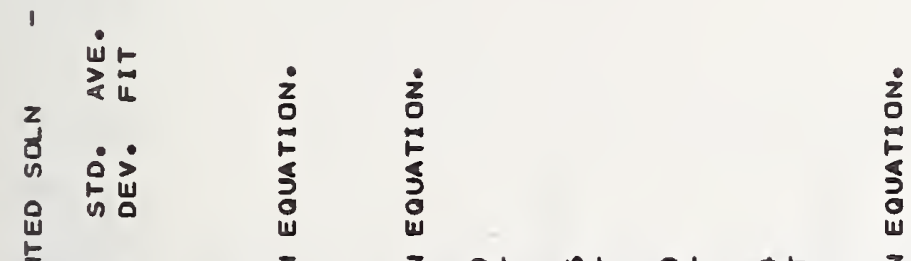

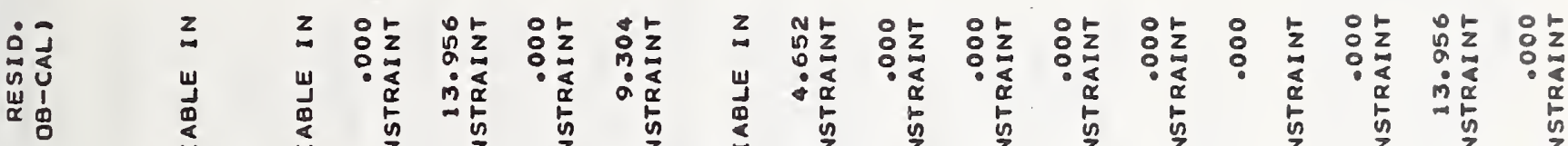

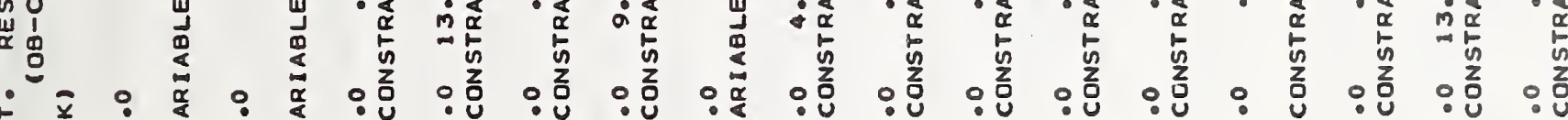

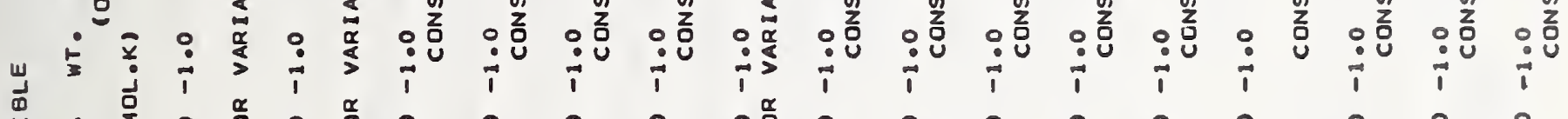

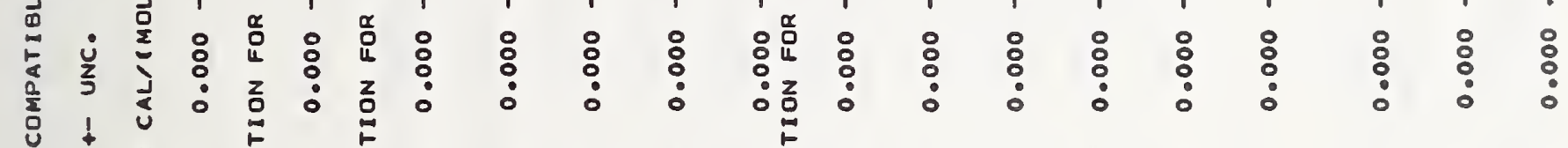

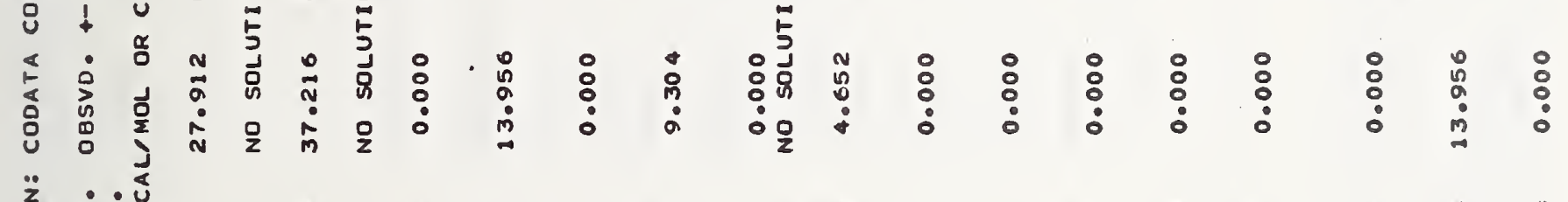

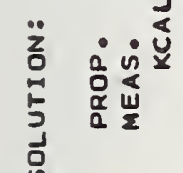

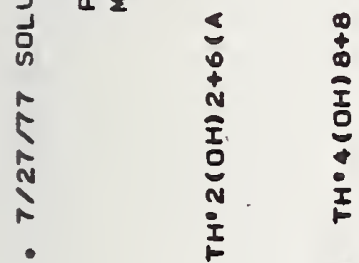

(a)

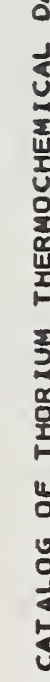

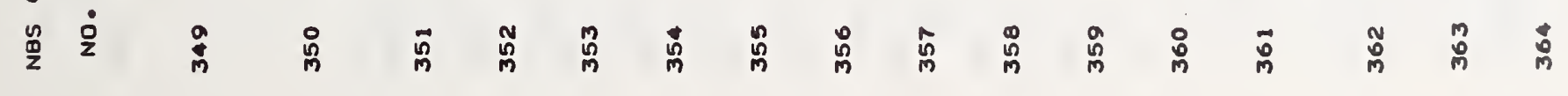




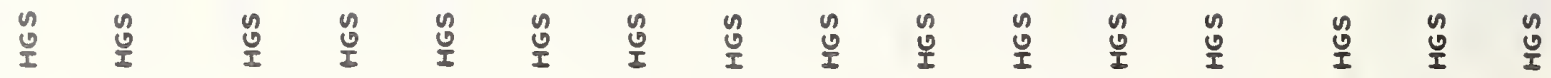

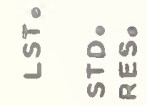

宸范

㝘离

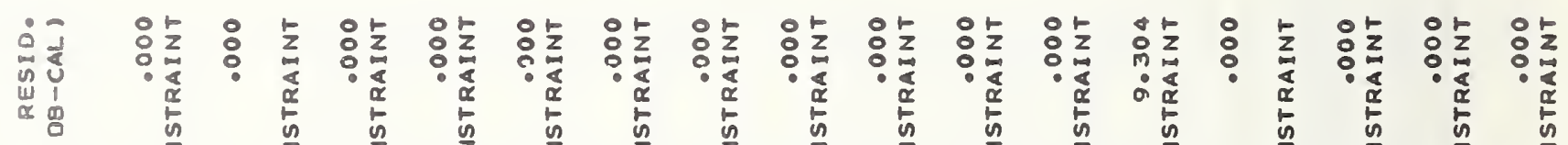

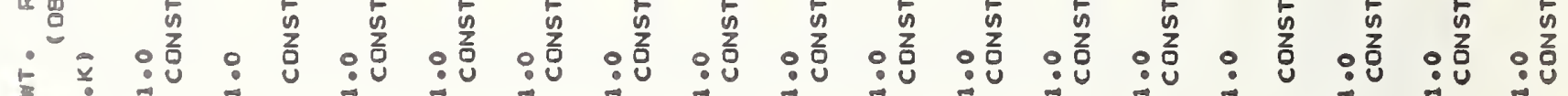

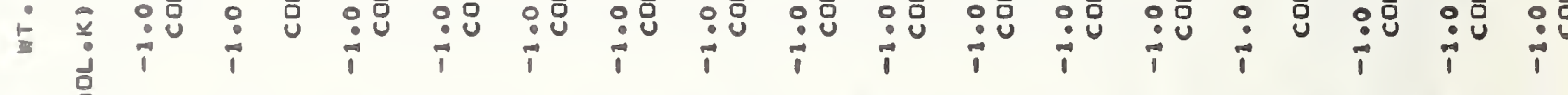

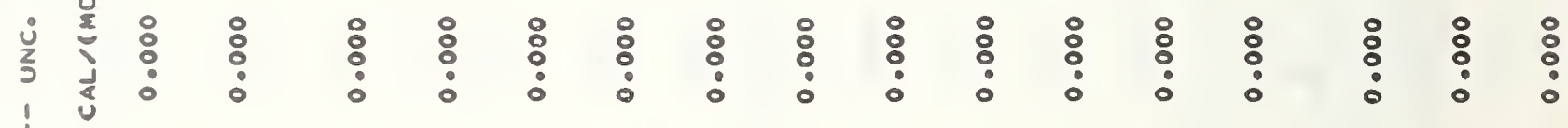

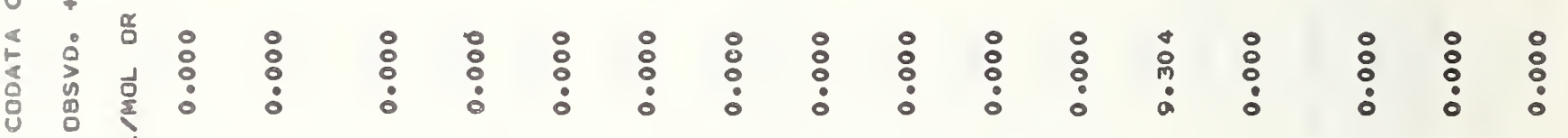

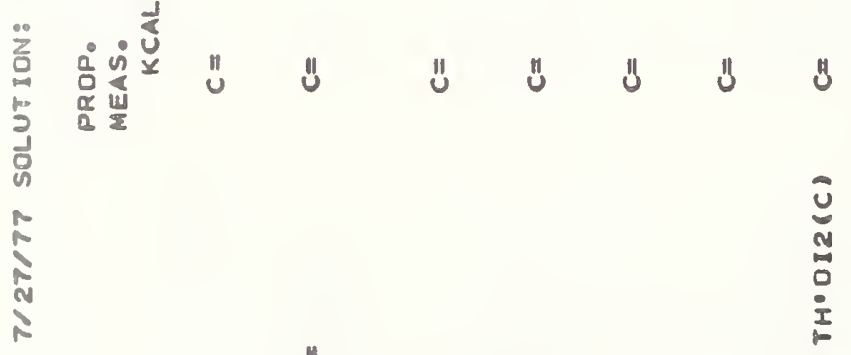




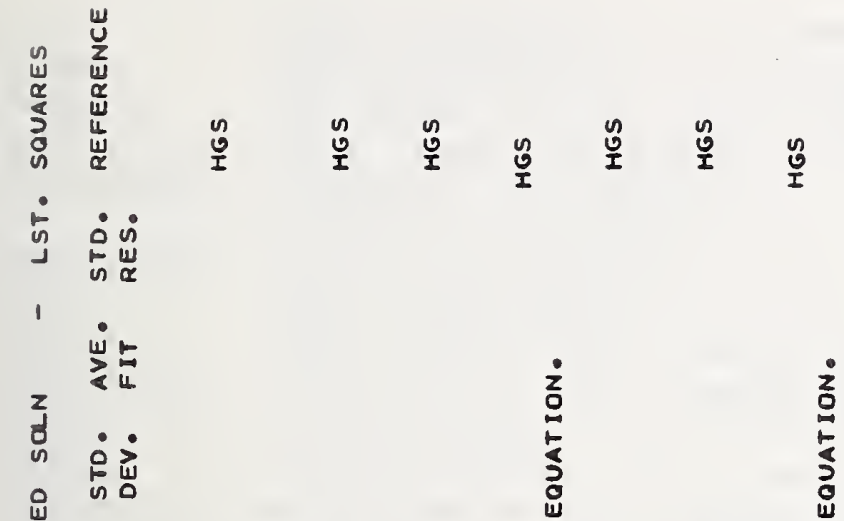

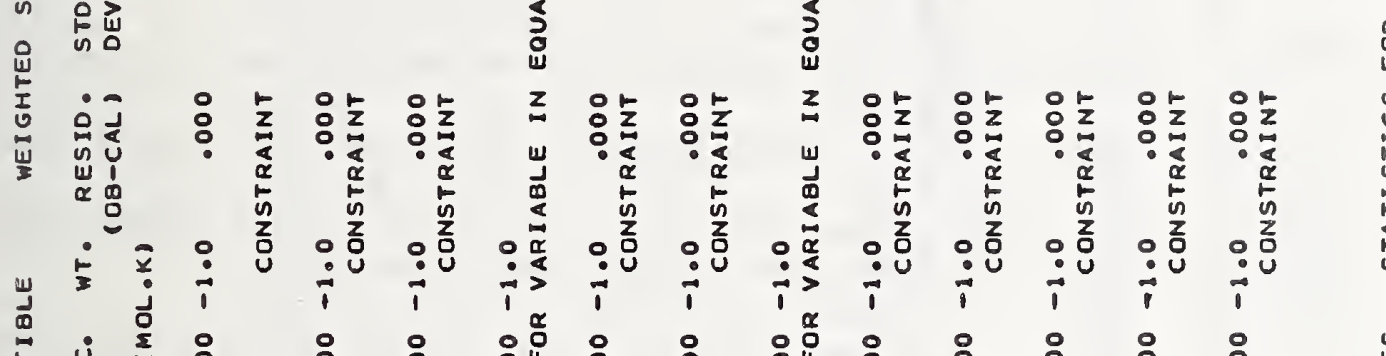

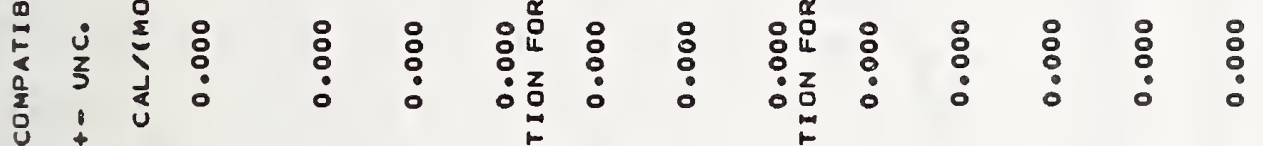

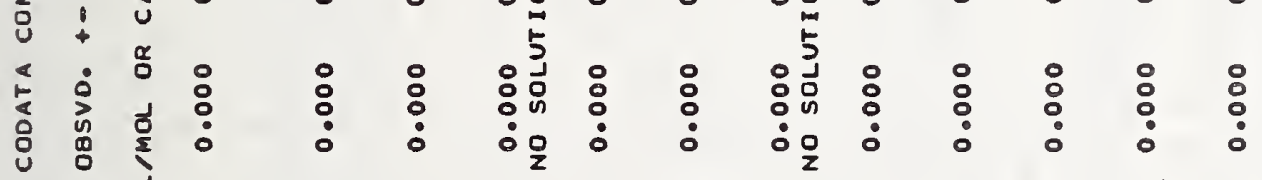

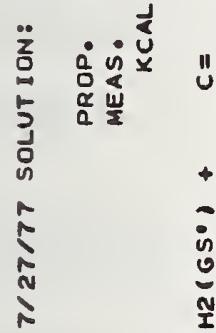

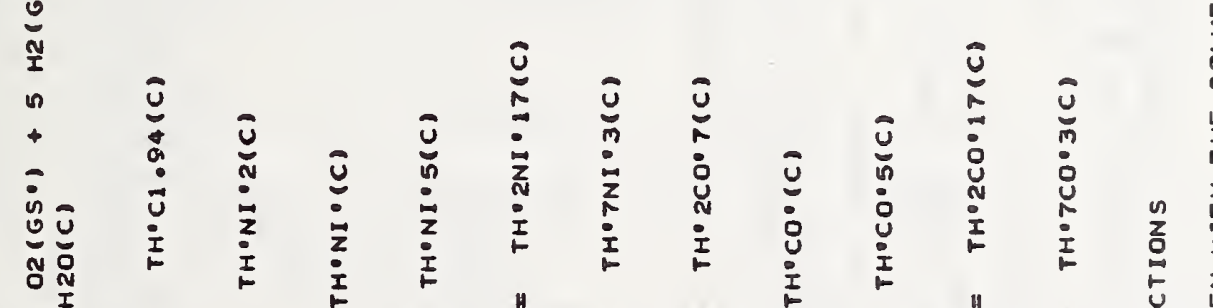

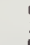

to

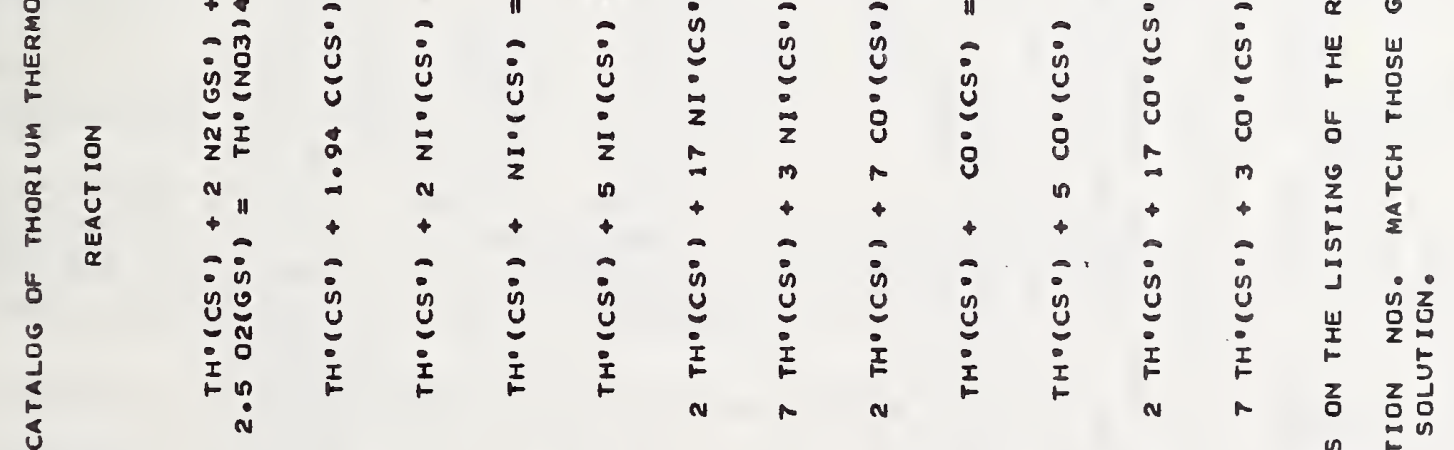

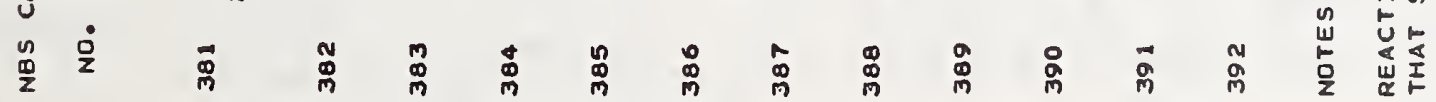

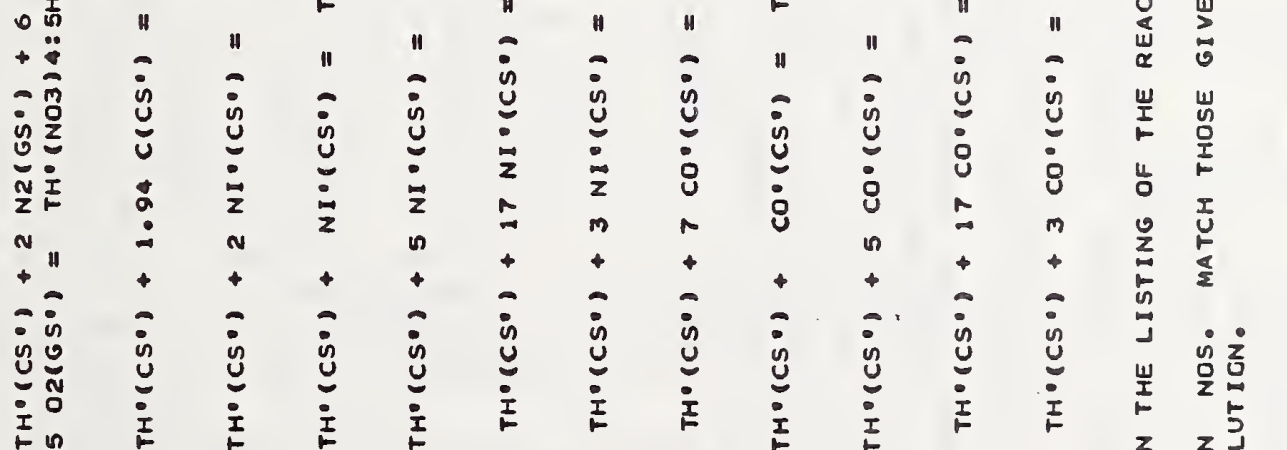




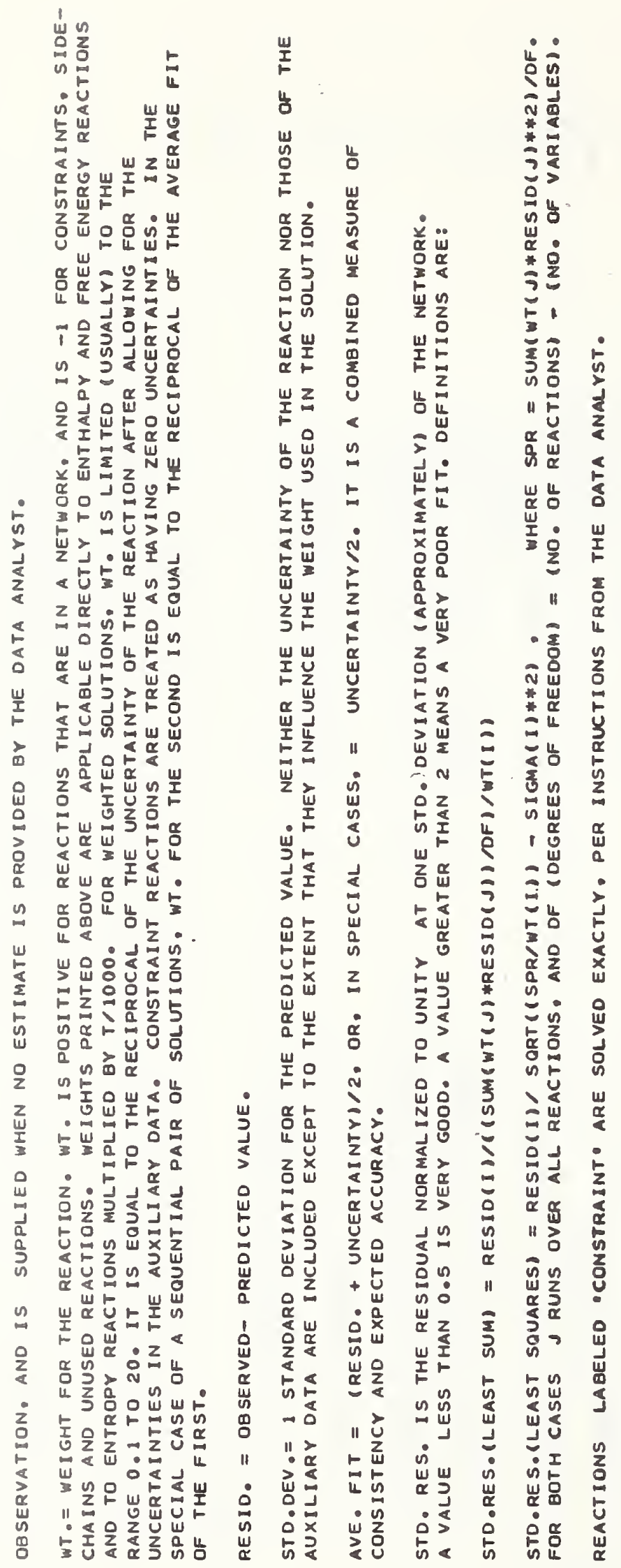


- N3S

O 9 $\mathrm{AAF}$

उOMIS

उZRCT/BEC

$34 N \equiv U / K R O$

39FIS/GEW

4 OBRA

4ISTR/ZUM

4 GGUG

$4 \in R O B / L E V$

49JOD/ROL

$49 W \equiv S / R O B$

SODAY/STO

$50 \equiv Y R / W E S$

SOUAG/STO

5 I MAL/CAM

51ZEB/ALT

52NOT/VIL

52WAG/STO $53 E Y R / W E S$

53GRI/5KO

$5335 B / W E S$ 54LOH/OSB

SARFA/HOL 55FER/KAT

55PAN/HSE 5 GFER/KAT

$570^{\circ} E / B O D$ 57LAN/MIE SBBEA/MCT 5 BDAR/KEN

59GAG/MAS

59KIN/WEL
EVALUATED DATA (SEE COMMENT ON REACTION) VON WARTENBERG. Ho: Z. ELEKTROCHEM. $15.866(1909)$ MISCIATELLI. P.: GAZZ. CHIM. ITAL.60.882(1930) ROTH. W.A.: BECKER, G.: Z. PHYSIK. CHEM.A 159. 1 (1932) NEUMANN. B.: KROGER. C.: KUNZ, H॰: Z. ANORG. ALLGEM. CHEM. 218. $379(1934)$

FISCHEF. W०: GEWEHR. R•: WINGCHEN, H•: Z. ANJRJ. ALLGEM• CHEN. 242. $161(1939)$

BRASELITEN. C.: COMPT. REND. $211.326(1940)$

STROTZER. E.F.: ZUMBUSCH. M.: Z. ANORG. ALLGEM. CHEM. 247. 415 (1941)

GUGGENHEIMER. K.M.: PROC. PHYS. SOC. LDNDON 58.456 (1946) ROBINSON. R.A.: LEVIEN. B.: TRANS. ROY. SOC. N.Z. 76.295 (1946)

DODGEN. H.W.: ROLLEFSON, G.K.: J. AM. CHEM. SOC. 71.2600 (1949)

WESTRUM. E.F.- JR.: ROBINSON. H.D.: THE TRASURANIUM ELEMENTS. NATL. NUCLEAR ENERGY SERIES, VOL 148 . PT 2.887 (MCGRAW-HILL. NEW YOFK. 1969)

DAY.R.A.. JR•: STOUGHTON. R.W.: J. AM. CHEM. SOC. 72.5662 (1950)

EYRING. L•: WESTRUM. E.F.. JR•: J. AM. CHEM. SDC. 72.5555 ( 1950$)$

WAGGENER. W.C.: STOUGHTON. R.W.: U.S. AEC REPORT CRNL $795(1950)$ MALLETT. M.W.: CAMPBELL. I.E.: J. AM. CHEM. SOC. 73.4850 (1951)

ZEBROSKI E EL•: ALTER, H.W.: HEUMANN . F.K॰: j. AM. CHEM. SOC. 73. 5646 (1951)

NOTTORF, R.W.: WILSON. A.S.: RUNDLE, R.E.: NEWTON. A.S•: POWELL. S.E.: IN KATZIN. L.I.: U.S. AEC REPORT TID-5223. 350 (1952)

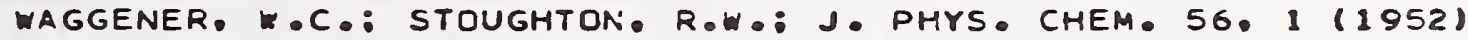
EYRING. L•: WESTRUM. E.F.• JR•: J. AM• CHEM: SOC. 75.4802 (1953)

GRIFFEL. M.: SKOCHDOPLE. R.E.: J. AM. CHEM. SOC.75. 5250 (1953)

OSBORNE.D.W.: WESTRUM, E.F..JR•: J. CHEM. PHYS. 21.1884 (1953) LOHR. H.R.: OSBORNE. D.*.: WESTRUM. E•F•• JR•: J. AM. CHEM. SOC. 76. 3837 (1954)

KRAUS. K.A.: HOLMBERG. R.W.: J. PHYS. CHEM. 5B. 325 (1954) FERRARO. S.R.: KATZIN. L.I.: QUOTED BY GILBREATH. ET AL. IN U.S. AEC REPORT ANL 5451. 89 PP. (1955)

PAN. K.: HSEU. T.M.: BULL. CHEM. SOC. JAPAN 28.162 (1955) FERRARO. S.R.: KATZIN. L.I.: GIBSON. G.: J. INORG. NUCL. CHEM. 2. $118(1956)$

D.EYE. R.W.M.: BOOTH. G.W.: J. INORG. NUCL. CHEM.4. 13 (1957) LANGE. E•: MIEDERER. W०: Z. ELEKTROCHEM. 61. 407 (1957) BEAR. J.: MCTAGGART. F.K.: AUSTRALIAN J. CHEM. 11.458 (1958) DARNELL. A.J.: KENESHEA.F.J.. JR.: J.PHYS. CHEM. 62. 1143 (1958)

GAGARINSKII. YU.V.: MASHIREV. V.P.: ZHUR. NEORG. KHIM. 4. 1246 (1959)

KING. E.G.: WELLER, W.W.: U.S. BUR. MINES. REPT. INVEST. 5485 5 PP. (1959) 
CSNIT

$5921 E$

SODAR I

GOJAF 2

GODAR/MCC

6OLAN/DAR

SOMAY/OWE

6 OWAL

OIBUC/SER

CIDAR/UCC

EIHOC/JOH 61ION/MIT EIVIC/DOU

S2VIK/IUK

S $3 A=K / R A U$

63 ALL/MCD

63 YEN/LI

$645 I N$

64JAC/SAR

6 4 NAB/KUD

653AE/MEY

65EDV/SEL

65LIN

GSSCA/TUR

S5WE S/TAK

G6ALC/COR

GGARD/AUS 66CHI/GAR

66GIN/ARO 6 GHEU/EGA

66!. EV

$66 S U / N O V$

$67 A R O$

CTBER

67MOS/ESS

67ILI/RUT

67 픈 MOR
MIT.KINA. E.A.: AT. ENERG. (RUSS.) 7. 163(2959): SOV. AT. ENERG. (ENGL.) 7.669. (1959)

ZIELEN. A.J.: J.AM. CHEM. SOC. B1. 5022 (1959)

DARNELL. A.J.: U.5. AEC NAA-SR 5045. 13 PP. (1960)

DARNELL. A.J.: J. INORG. NUCL. CHEM. 15.359(1960)

DARNELL. A.J.: MCCOLLUM. W.A.: MILNE. T.A.: J. PHYS. CHEM. 64. $341(1960)$

LANDIS. A.L.: OARNELL. A.J.: U.S. AEC REPORT NAA-SR-5394. 15 PP (1960)

MAYER, S.W.: OWENS. B.B.: RUTHERFORD. T.H.: SERRINO.R.B.: 1. PHYS. CHEM. 64. 911 (1960)

WALLACE. D.C.: PHYS. REV. 120.84 (1960)

BUCHLER,A.: BERKOWITZ-MATTUCK.J.B.: DUGRE.D.H.: J. CHEM. PHYS. 34. 2202 (1961)

DARNELL. A.J.: MCCQLLUM. W.A.: AEC FEPORT NAA-SR-6498. 144PP.. (1961)

HOCH.M.: JOHNSTON.H.L.: J.PHYS. CHEM. 65. 1184 (1961) IONOV. V.I.: MITTSEV. M.A.: SOV.PHYS. JETP 13.518 (1961). VICTOR. A.C.: DOUGLAS. T.B.: J. RESEARCH NAT. BUR. STAND. 65A. 105 (1961)

NIKOLAEV. N.S.: LUKYANYCHEV. YU. A.: SOV.J. AT. ENERGY (USSR) 12. $334(1962)$

ACKERMANN, R.J.: RAUH, E.G.: THCRN, R.J.: CANNON, M.C.: J. PHYS. CHEM. 67.762 (1963)

ALLEN. K.A.: MCDOWELL. W.J.: J.PHYS. CHEM. 67. 1138 (196.3) YEN. K. F.: LI. S.-C.: NOVIKOV. G. I.: RUSS.J.INORG. CHEM. 8. 48 (1963)

GINGERICH, K.A.: U.S. AEC REPORT NYO2541-1 (1964)

JACKSON. D.D.: BARTON, G.W.. JR.: KRIKORIAN. O.H.: NEWBURY. R.S.: J.PHYS. CHEM. 68. 1516(1964)

NABIVANETS. B.I.: KUDRITSKAYA. L.N.: UKR. KHIM. ZH. 30. 891 (1964)

BAES, C.F.. IR.: MEYER. N.J.: ROBERTS. C.E.: J. INORG. CHEM. 4. $518(1965)$

EDVINSSON, G.: SELIN. L.E.: ASLUND. N.: ARKIV FYSIK. 30. $283(1965)$

LINEVSKY. M.: CHEM. PROPULSION INFORM. AGENCY THERMJCHEM. PROCEEDINGS 1.71 (1965)

SCAIFE, D.E.: TURNBULL. A.G.: WYLIE. A.W.: J. CHEM. SOC. (LONDON) 1965. 1432

WESTRUM. E.F.• JR•: TAKAHASHI. Y॰: STOUT. N.D.: J• PHYS. CHEM. 69. $1520(1965)$

ALCDCK. C.B.: CORNISH. J.B.: GRIEVESON.P.: -THERMDDYNAMICS* VOL I. P.211 (INT. AT. ENERGY AGENCY. VIENNA. 1966)

ARONSON. S.: AUSKERN. A.B.: J.PHYS. CHEM. 70.3937 ( 1966$)$ CHIOTTI.P.: GARTNER,G.J.: STEVENS.E.R•: SAITO.Y.: J. CHEM. ENG. DATA 11. 571 (1966)

GINGERICH, K.A.: ARONSON. S.: J.PHYS. CHEM.70. 2517 (1966) HEUS. R.J.: EGAN, J.J.: Z. PHYSIK. CHEM. (NF) 9.38 (1966) LEVINSON. L.S.: J. NUCL. MATER. 19.50 (1966)

SU. M.T.: NOVIKOV. G.I.: ZHUR. NEORG. KHIM. 11.498 (1966) ARONSON, S.: J. INORG. NUCL. CHEM. 29.1611 (1967)

BERAN. M.: COLL. CZECH. CHEM. COMMUN. 32. 1368 (1967)

MOSKVIN. A. I.: ESSEN. L.N.: ZHUR. NEORG• KHIM. 12, 698 (1967)

ILINA. G.G.: RUTGAIZER. YU. S.: SEMENOV. G.A.:

PRIBORY I TEKHNIK EKSPER. 1. 151 (1967)

WEED. H.C.: MORROW. R.J.: U.S.AEC REPORT UCRL-7O245. 26 PP. ( 1967$)$ 
UEHIE/SIL E MHUE/ TOL

G BDHA/MDR

GBZAL

69GIN

6 QGUE/PED

69LOR/SCH

69MUC/5MI

6SSMI/HER

69SMI/THA

7 C.SAU

7 OSRY/ROG

$7 C D E V / R U D$

7OGFE/BRY

7 OIUP

$70 M 00$

7 CFOS

$7 O S E I / A S T$

7 OSKE/MAG

$70 Z M B$

7 IFLO/JSB

7 IKLO/MUK

$71 \mathrm{KUS} / \mathrm{IMO}$

7 IL AU/FOU

$71 \mathrm{MIL}$

$715 \mathrm{KE} / \mathrm{MAG}$

72ACK/RAU

$72 A D \equiv / H U B$

720 AN/NOV

72KNA/MUL 1

$72 K$ VA/MUL 2

$72 T E D / A N O$
HIETANEN, S.: SILLEN. L.G.: AETA. CHEN. SCAND. Z2. 265 (19t8) HUBER. E.J.. JR.: HOLLEY. C.E.. JF.: KRIKORIAN. N.H.: J. CHEM. ENG. CATA $13.253(2968)$

OHASHI. H.: MOROZUMI. T.: NIPPON GENSHIRYOKU GAKKAI 10. $244(1968)$

ZALUBAS. R.: J. OPT. SOC. AMER. 58. 1195 (1968)

GINGERICH. K.A.: HIGH TEMP. SCI. 1.258 (1969)

GUEST. M. F.: PEDLEY. J. B.: AND HORN, M.: J. CHEM.

THERMODYNAMICS 1.345 (1969).

LORENZ, K.: SCHERFF. H.G०: TOUISSANT. N.T.: J. INORG. NUCL. CHEM. 31. 2381(1969)

MUCKEN. K.: SMITH. G.S.: JOHNSON. O.: ELSON. R.C.: ACTA CRYST. B25. $236(1969)$

SMITH. D.H.: HERTEL. G.R.: J. CHEM. PHYS. 51. 3105 (1969)

SMITH, B.C.: THAKUR, L.: WASSEF, M.A.: INDIAN J. CHEM. 7.1154

( 1969$)$

BAUMANN. E.W.: J. INORG. NUCL. CHEM. 32..3823 (1970)

BRYZGALOVA. R.V.: ROGOZIN. YU. M•: CHERNITSKAYA. I.V.:

RADIOKHIMIYA 12.286 (1970)

VAN DEVENTEF, E.H.: RUDZITIS. E.: HUBBARD. W.N.: J. INORG. NUCL. CHEM. 32. 3233 (1970)

GREBENSHCHIKOVA. V.I.: BRYZGALOVA. R.V•: ROGOZIN•YU. M.:

FADIOKHIMIYA 12.279 (197C)

COMMISSION ON ATOMIC WEIGHTS DF IUPAC. PURE APPL. CHEM.

21. $91(1970)$.

MOORE. C.E.: IONIZATION POTENTIALS AND IONIZATION LIMITS DERIVED FROM THE ANALYSES OF OPTICAL SPECTRA. NSRDS-NBS34 6 PP. (SEPT. 1970 )

ROSEN, B.: SPECTROSCOPIC DATA RELATIVE TO DIATOMIC MOLECULES. (PERGAMON PFESS. ELMSFORD. N.Y. 1970)

SEIGEEV. G.M.: ASTRASHKOVA. L.G.: YAGODINSKAYA. N.N.:

RADIOKHIMIYA 12.392 (1970)

SKELTON. W.H.: MAGNANI. N.J.: SMITH. J.F.: MET. TRANS• 1. $1833(1970)$

ZMBOV. K.F.: J. INORG. NUCL. CHEM. 32.1378(1970)

FLOTOW, H.E.: OSBORNE. D.W.: WALTERS.R.R.: J. CHEM.PHYS. 55. 880 (1971)

KLOTZ. P.: MUKHERJI. A.: FELDBERG, S॰: NEWMAN. L•: INORG. CHEM. 10.740 (1971)

KUSAKABE. T.: IMOTO. 5.: NIPPON KINZOKU GAKKAISHI 35. 1115 ( 1971 )

LAUBSCHER. A.E.: FOUCHE.K.F•: J. INORG. NUCL. CHEM. 33. $3521(1971)$

MILIC. N.B.: ACTA CHEM. SCAND.25. 2487 (1971)

SKELTON. W.H.: MAGNANI. N.J.: SMITH, J.F•: MET• TRANS. 2. $473(1971)$

ACKERMANN, R.S.: FAUH, E.G.: J. CHEM. THERMODYNAMICS 4. $521(1972)$

ADER, M.: HUBBARD. W.N.: O०HARE.P.A.: U.S. AEC REPOZT ANL 7876-1. 33 PP. (1972)

DANAN, J.: DE NOVION, C.H०: DALLAPORTA. H.: SOLID STATE COMMUN. 10. $775(1972)$

KNACKE, 0.: MULLER, F.: VAN RENSEN, E•: Z. PHYSIK. CHEM. (NF) 80. 82 (1972)

KNACKE, O.: MULLER, F.: VAN RENSEN, E•: Z. PHYSIK. CHEM. (NF) 80. 91 (1972)

TEDESCO. P.H.: ANON. M.C.: J. INORG. NUCL. CHEM. 34. 2271 ( 1972 ) 
$72 U S H / 5 K O$

$72 W \equiv N / S P I$

73ACK/RAU

7 ЗAPE/AZO

$73 C D D$

7 BHUL/OES

7 $3 K F A / M O R$

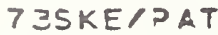

7 SUG

$7430 \mathrm{H} /<$ RA

$74 G A B / Z E E$

$74 H I L / M U R$

$74 N E U / Z M B$

$742 A U / A C K$

$75 C O D$

75PAR/WAG

TSRAN

$76 C H E / W E S$

76GAR/PAR

7 GMOR/MCC 7TFLC
USHERENKO, L.N.: SKORIK, N.A.: RUSS. J. INORG. CHEM. 17 . $1533(2972)$

WENTINK. T.. JR : : SPINDLER, R.J.: J. OUANT. SPECTROSC. RADIAT. TRANSFER 12, 1569 (1972)

ACKERMANN, R.J.: RAUH, E.G.: HIGH TEMP. SCI. 5. 463 (1973) ANELBLAT. A.: AZOULAY, D.: SAHAR. A.: J. CHEM. SOC FARADAY TRANS. I. 1973,1618

CODATA TASK GROUP ON FUNDAMENTAL CONSTANTS, CODATA BULLETIN NO. 11 (DECEMBER 1973 ).

HULTGREN, F•: DESAI. P.D.: HAWKINS, D.T.: GLEISER, M.:

KELLEY. K.K.: WAGMAN. D.D.: 'SELECTED VALUES THERMODYN. PROP. ELEMENTS' ( 1573 ) PUBL. BY AM. SOC. METALS

KRASNOV. K.S.: MOROZOV. E.V.: FILLIPENKO. N.V.: GIRICHEVA. N.I.: IZV. VYSSH, UCHEB. ZAVED. KHIM. I KHIM. TEKHNOL. 16. 1500 (1973)

SKELTON, W.H.: PATTEFSON, J.W.: J. LESS COMMON METALS 31.47 (1973)

SUGAR. J.: J. CHEM. PHYS. 59.788 (1973)

BOHRES. E.W.: KRASSER, W.K.: SCHENK. H.J.: SCHWOCHAU, K. J. INORG. NUCL. CHEM. 36.889 (1974)

GABELNICK.S.D.: REEOY.G.T.: CHASANOV.M.G.: \&. CHEM. PHYS. 60.1267 (1974)

HILDENBRAND, D.L.: MURAD, E.: J. CHEM. PHYS. 61. 1232 (1974) NEUBERT. A.: ZMBOV. K.F.: HIGH TEMP. SCI. 6. 303 (1974)

FAUH. E.G.: ACKERMANN. R.J.: J. CHEM. PHYS. 60. 1396 (1974) CODATA RECOMMENDED KEY VALUES FOR THERMODYNAMICS 1975. CODATA BULL. NO. 17 (JAN. 1976). AND CODATA SPECIAL REPORT NO. 3 (SEPT. 1975), CODATA, PARIS. PARKER, $V$. B: WAGMAN, D. D.: AND GARVIN. D.:

NAT. BUR. STAND. (U.S.) NBSIR 75-968. 34 PP. (1976)

RAND. M.: AT. ENERGY REV. SPECIAL ISSUE NO. 5.

IAEA, VIENNA (1975).

CHEOA.J.A.R.: WESTRUM.E.F..JF.: J.CHEM.THERMDDYNAMICS 8.25 (1976)

GARVIN, D.: PARKER, V. B.: WAGMAN, D. D.: AND EVANS. W. H.: NAT. BUR. STAND. (U.S.). NBSIR 76-1147. 37 PP. (1976)

MORSS. L.R॰: MCCUE. M.C.: J. CHEM. ENGR. DATA 21.337 (1976)

FLOTOW.H.. ARGONNE NATL.LAB.. PRIVATE COMMUNICATION. MARCH 1977 
IV. APPENDIX 
. 
In reducing high-temperature equilibrium measurements to values at $298 \mathrm{~K}$ we have made use of the thermal functions $\mathrm{S}^{\circ}$, $\left(H^{\circ}-H^{\circ}(298)\right)$, and $G^{\circ}-H^{\circ}(298) / T$ for a number of thorium compounds. This section of the Report contains tables of values of the functions that have been used and gives sources of data from which the tables are derived.

Th(c) The high-temperature enthalpy measurements of Levinson [66 LEV] from 1260-2100 $\mathrm{K}$ were fitted empirically to the following equations, which also reproduce the value of $\mathrm{Cp}$ at $298 \mathrm{~K}$ :

$T h(c, \alpha): C p=6.267 \pm 0.000664 \mathrm{~T}+7.02 \times 10^{-7} \mathrm{~T}^{2} \mathrm{cal} / \mathrm{mol} \cdot \mathrm{K}(298-1660)$

Transition $(c, \alpha)=(c, \beta): \Delta H=870 \mathrm{cal} / \mathrm{mol}$ at $1653 \mathrm{~K}$

$\operatorname{Th}(\mathrm{c}, \beta): \mathrm{Cp}=3.72+0.00288 \mathrm{~T} \mathrm{cal} / \mathrm{mol} \cdot \mathrm{K}$

Fusion $(c, \beta)=(1): \Delta H=3300 \mathrm{cal} / \mathrm{mol}$ at $2023 \mathrm{~K}$

$\operatorname{Th}(1): \quad C_{p}=11.0 \mathrm{cal} / \mathrm{mol} \cdot \mathrm{K}$

The above equations for Th $(c, \alpha)$ yield $C p$ values significantly lower than those reported by Wallace [6OWAL] and Mitkina [59MIT] and reproduce the values of Levinson on $T h(c, \alpha)$ more closely than the equation given by Rand [75RAN].

Th(g) Calculated from the 75 energy levels to $20,000 \mathrm{~cm}^{-1}$ given by Zalubas [68ZAL]. Up to $2500 \mathrm{~K}$ these values are essentially the same as those given by Rand [75RAN].

Tho(g) Calculated from the molecular constants given by Rosen [70ROS], using a non-rigid rotator, anharmonic oscillator approximation and including contributions from the higher electronic levels. This analysis of the higher levels is different from that used by Rand [75RAN], and consequently our vafues of $\mathrm{S}^{\circ}$ are lower than Rand's by $0.01,0.03$, and $0.54 \mathrm{cal} \cdot \mathrm{K}^{-1} \cdot \mathrm{mol}^{-1}$ at 298,1000 , and $2000 \mathrm{~K}$, respectively. 
$\mathrm{ThO}_{2}$ (c) The entropy and enthalpy content at $298 \mathrm{~K}$ are based on the measurements of Osborne and Westrum [53 OSB/WES]. The high temperature enthalpy data of Victor and Douglas [61 VIC/DOU] $(273-1173 \mathrm{~K})$ and Hoch and Johnston [61 HOC/JOH] (1450-2750 K) are well represented by the equation given by Victor and Douglas. The data of Hoch and Johnston show considerable scatter with respect to any simple analytical function in $T$. The equation used to calculate the thermal functions is:

$$
\mathrm{Cp}\left(\mathrm{cal} \cdot \mathrm{K}^{-1} \cdot \mathrm{mol}^{-1}\right)=17.060+0.001806 \mathrm{~T}-\frac{251660}{\mathrm{~T}^{2}}
$$

$\mathrm{ThO}_{2}(\mathrm{~g})$ The molecular constants are taken from Gabelnick et al. [74 $\mathrm{GAB} / \mathrm{REE}$ ] who demonstrated by isotopic substitution that the gaseous molecule is bent with an apex angle of $122^{\circ}$. The low lying frequency is taken as $120 \mathrm{~cm}^{-1}$, based on an estimate by Linevsky [65 LIN].

The available vapor pressure data on $\mathrm{ThO}_{2}$ are not easily reconciled with the available thermal functions but do satisfy functions for $\mathrm{ThO}_{2}(\mathrm{~g})$ as a linear molecule.

$\operatorname{ThX}_{\mathrm{n}}(\mathrm{g})(\mathrm{X}=\mathrm{F}, \mathrm{Cl}, \mathrm{Br}, \mathrm{I}, \mathrm{n}=1,2,3)$ The values of $\omega_{\mathrm{e}}$ for $\operatorname{ThX}(\mathrm{g})$ were estimated using the Guggenheimer equation [46 GUG]; we have estimated the anharmonicity constant $\omega_{e} x_{e}$. The degeneracy of the ground state is taken $=4$. Values of $r_{e}$ are taken from Krasnov et a 1 [73 KRA/MOR], who also give values for $\omega_{e}$.

For $\operatorname{ThX}_{2}(g)$ and $\operatorname{ThX}_{3}(g)$ we have used the molecular constants and frequencies estimated by Krasnov et a1. [73 KRA/MOR] for a rigid-rotator-harmonic oscillator. As the ground state of $\left.\mathrm{ThO}_{\mathrm{g}} \mathrm{g}\right)$ was shown to be $1 \Sigma$, we have taken the degeneracy of the ground state of the dihalides to be unity, whereas we have estimated the degeneracy as 6 for the trihalides $\operatorname{ThX}_{3}(\mathrm{~g})$.

$\mathrm{ThF}_{4}$ (c) Values of $\mathrm{S}^{\circ}$ and $\mathrm{H}-\mathrm{H}(0)$ at $298 \mathrm{~K}$ are based on the measurements of Lohr et al. [54 LOH/OSB]. Preliminary unpublished high temperature enthalpy measurements by Dworkin [74 DWO] from $1200 \mathrm{~K}$ $1420 \mathrm{~K}$ were used to obtain approximate values of the enthalpy of fusion and heat capacities. The equations used are:

$$
\begin{aligned}
& \mathrm{Cp}(\mathrm{c})=29.2+0.002 \mathrm{~T}-300000 / \mathrm{T}^{2} \mathrm{cal} / \mathrm{mol} \cdot \mathrm{K} \\
& \triangle \mathrm{H}(\text { fusion })=10000 \mathrm{cal} / \mathrm{mol} \text { at } 1383 \mathrm{~K} \\
& \mathrm{Cp}(1)=32 \mathrm{ca} 1 / \mathrm{mol} \cdot \mathrm{K}
\end{aligned}
$$


$\mathrm{ThF}_{4}(\mathrm{~g}) \quad$ Molecular constants have been estimated by Krasnov et al. [73 $\mathrm{KRA} / \mathrm{MOR}]$ and by Rand [75 RAN]. Calculations using both sets

of frequencies have been made with the vaporization data of Darnell and Keneshea [58 DAR/KEN]. A better fit (Second Law and Third Law] is obtained with Rand's function so these are adopted here.

ThC14(c) There are no good low temperature heat capacity data for $\mathrm{ThCl}_{4}(\mathrm{c})$. The measurements by Chauvenet [11 CHA] near room temperature are unreasonable. We have estimated $\mathrm{S}^{\circ}(298 \mathrm{~K})=$ $45.5 \mathrm{cal} \cdot \mathrm{K}^{-1} \cdot \mathrm{mol}^{-1}$ based on comparisons with $\mathrm{HfCl}_{4}(\mathrm{c}), \mathrm{ZrCl}_{4}$ (c), $\mathrm{UCl}_{4}(\mathrm{c})$, and the $\mathrm{UF}_{4}(\mathrm{c})-\mathrm{ThF}_{4}$ (c) difference. We have accepted Rand's heat capacity equation [75 RAN].

The enthalpy and temperature of transition and fusion were determined by Chiotti et a1. [66 CHI/GAR].

The equations used are:

$$
\begin{aligned}
& \mathrm{Cp}(\mathrm{c})=28.75+0.005561 \mathrm{~T}-147000 / \mathrm{T}^{2} \mathrm{cal} / \mathrm{mol} \cdot \mathrm{K}[298-1042 \mathrm{~K}] \\
& \Delta H(\text { transition })=1200 \mathrm{cal} / \mathrm{mol} \text { at } 679 \mathrm{~K} \\
& \Delta H(\text { fusion })=14690 \mathrm{cal} / \mathrm{mol} \text { at } 1042 \mathrm{~K} \\
& \mathrm{Cp}(1)=40 \mathrm{cal} / \mathrm{mol} \cdot \mathrm{K}
\end{aligned}
$$

$\mathrm{ThCl}_{4}(\mathrm{~g})$ The thermal functions for $\mathrm{ThCl}_{4}(\mathrm{~g})$ are calculated assuming a tetrahedral molecule (symmetry $=12$ ), ground state degeneracy $=1$, and the $\mathrm{Th}-\mathrm{Cl}$ bond distance $=2.58 \AA$. The frequencies and degeneracies are taken to be $300(1), 70(2), 335(3), 75(3)$. The frequency at $335 \mathrm{~cm}^{-1}$ has been observed by Buchler et al. [61 BUC/BER]. The enthalpy and entropy of vaporization data computed from the measurements of Knacke et al. [72 KNA/MUL1] are consistent with the functions given here. The data of Fischer et a1. [39 FIS/GEW] on the vapor pressure of $\mathrm{ThCl}_{4}$ (1) and (c) are not very consistent with the selected functions. 
$\operatorname{ThBr}_{4}$ (c) The heat capacity of the solid and liquid is based on estimated constants given by Rand [75 RAN]. The enthalpy of fusion is calculated from the vapor pressure data of Fischer et al. [39 FIS/GEW]. The entropy is estimated. The equations used are:

$$
\begin{aligned}
& \mathrm{Cp}(\mathrm{c})=30.5+0.0036+-147000 / \mathrm{T}^{2} \mathrm{cal} / \mathrm{mol} \cdot \mathrm{K}[298-952 \mathrm{~K}] \\
& \triangle \mathrm{H}(\text { transition })=1000 \mathrm{cal} / \mathrm{mol} \text { at } 693 \mathrm{~K} \\
& \triangle H(\text { fusion })=7000 \mathrm{ca} 1 / \mathrm{mol} \text { at } 952 \mathrm{~K} \\
& \mathrm{Cp}(1)=41 \mathrm{ca} 1 / \mathrm{mol} \cdot \mathrm{K}
\end{aligned}
$$

$\mathrm{ThBr}_{4}(\mathrm{~g})$ The functions are calculated for a tetrahedral molecule with $\mathrm{Th}-\mathrm{Br}$ distance $=2.72 \AA$ and frequencies $205(1), 58(2), 220(3)$, $72(3)$, as estimated by Rand [75 RAN]. The vapor pressure data of Fischer et al.[39 FIS/GEW] are consistent with these functions for both solid and liquid.

$\mathrm{ThI}_{4}$ (c) The thermal functions for the solid and liquid were calculated from the estimated heat capacity and enthalpy of fusion data given by Rand. The vapor pressure of the solid has been reported by Landis and Darne11 [60 LAN/DAR; equation on 1y]; data for the liquid are given by Fischer et al. [39 FIS/GEW]. The two sets of data do not agree at the triple point, and do not yield similar values for the heat of sublimation at $298 \mathrm{~K}$. If it is assumed that the Landis and Darnell equation, $\log \mathrm{P}(\mathrm{atm})=10.32-10700 / \mathrm{T}$ should be $\log \mathrm{P}(\mathrm{atm})=10.32-10300 / \mathrm{T}$ (misprint in the report?), both sets of data yield consistent values and indicate that $S^{\circ}(298)$ $=61 \mathrm{cal} \cdot \mathrm{K} \cdot \mathrm{mo} 1^{-1}$.

The equations used are:

$$
\begin{aligned}
& \mathrm{Cp}(\mathrm{c})=31.0+0.0031 \mathrm{~T}-147000 / \mathrm{T}^{2} \mathrm{ca} 1 / \mathrm{mol} \cdot \mathrm{K}[298-839 \mathrm{~K}] \\
& \triangle \mathrm{H}(\text { fusion })=11500 \mathrm{ca} 1 / \mathrm{mol} \text { at } 839 \mathrm{~K} \\
& \mathrm{Cp}(1)=42 \mathrm{ca} 1 / \mathrm{mol} \cdot \mathrm{K}
\end{aligned}
$$


$\mathrm{ThI}_{4}(\mathrm{~g})$ The functions are calculated from the molecular constants and frequencies given by Krasnov et a1. [73 KRA/MOR]. These frequencies satisfied the relations given by Herzberg [ 45 HER] better than did those given by Rand [75 RAN]. 
$\operatorname{Th}(c, I)$

$T$

$\begin{array}{cl}\text { CP } & H-H 298 \\ C A L / M O L \cdot K & C A L / M O L\end{array}$

$6 \cdot 53$

6.53

6.64

6.77

6.92

7.08

7.25

7.43

7.63

7.85

8.67

8.32

8.57

8.84

9.13

9.28

$H T=870 \mathrm{CAL} / \mathrm{MOL}$
1653
1700
1800
1900
2000
2023

$H T=3300 \mathrm{CAL} / \mathrm{MOL}$

2023
2100
2200
2300
2400
2500
2600
2700
2800

11.00

11.00

11.00

11.00

11.00

11.00

11.00

11.00

11.00

0.
$12^{\circ}$
$671^{\circ}$
$1342^{\circ}$
$2026^{\circ}$
$2726^{\circ}$
$344{ }^{\circ}$
$4176^{\circ}$
$4929^{\circ}$
$57030^{\circ}$
$6499^{\circ}$
$7318^{\circ}$
$8162^{\circ}$
$9033^{\circ}$
$9931^{\circ}$
$10419{ }^{\circ}$

12.76

12.80

14.69

16.19

17.44

18.52

19.47

20.34

$21 \cdot 13$

21.87

22.56

23.22

23.84

24.44

25.02

$25 \cdot 32$

$S T=.52632 \mathrm{CAL} / \mathrm{MOL} \cdot \mathrm{K}$

$11289^{\circ}$
$11691^{\circ}$
$12567^{\circ}$
$13472^{\circ}$
$14405^{\circ}$
14624.

25.85

26.09

26.59

27.68

27.56

27.66

$S T=1.6312 \mathrm{CAL} / \mathrm{MOL} \cdot \mathrm{K}$

17924.
$18771^{\circ}$
$19871^{\circ}$
$20971^{\circ}$
$22071^{\circ}$
$23171^{\circ}$
$24271^{\circ}$
$25371^{\circ}$
$26471^{\circ}$

$29 \cdot 30$

29.71

30.22

30.71

31.18

31.62

32.06

$32 \cdot 47$

$32 \cdot 87$
( G-H298) /T

CAL/MOL .K

$-12 \cdot 76$

$-12.76$

$-13.02$

$-13.51$

$-14.06$

$-14.62$

$-15.17$

$-15 \cdot 70$

$-16.20$

$-16.68$

$-17.14$

$-17.59$

$-18.01$

$-18 \cdot 42$

$-18.81$

$-19.02$

AT $1653 \mathrm{~K}$

$-19 \cdot 02$

$-19.21$

$-19.61$

$-19.99$

$-20 \cdot 35$

$-20 \cdot 44$ 
Th （g）

T

$\begin{array}{ll}298 & 4.969 \\ 300 & 4.969 \\ 400 & 4.982 \\ 500 & 5.039 \\ 600 & 5.171 \\ 700 & 5.388 \\ 800 & 5.679 \\ 900 & 6.024 \\ 1000 & 6.398 \\ 1100 & 6.780 \\ 1200 & 7.154 \\ 1300 & 7.509 \\ 1400 & 7.837 \\ 1500 & 8.138 \\ 1600 & 8.409 \\ 1700 & 8.653 \\ 1800 & 8.870 \\ 1900 & 9.063 \\ 2000 & 9.235 \\ 2100 & 9.388 \\ 2200 & 9.523 \\ 2300 & 9.642 \\ 2400 & 9.746 \\ 2500 & 9.837 \\ 2600 & 9.915 \\ 2700 & 9.980 \\ 2800 & 10.034 \\ 2900 & 10.077 \\ 3000 & 10.109\end{array}$

$H-H(\theta)$

CAL/MOL

CAL/MOL.K

1481.2

1490.4

1987.7

2488.2

2998.0

3525.3

$4878 \cdot 1$

4662.9

5283.9

$5942 \cdot 8$

$6639 \cdot 7$

7373.8

8140.5

8939.5

9767.1

10620.5

11496.8

12393.7

$13368 \cdot 8$

14240.1

15185.7

16144.0

17113.5

18092.8

19080.5

20075.3

21076.1

$22081 \cdot 8$

23091.2
$\stackrel{S}{C A L / M O L} \cdot K$

45.424

45.454

46.885

48.062

48.931

$49 \cdot 743$

50.481

$51 \cdot 169$

$51 \cdot 823$

52.451

53.057

53.644

54.212

54.763

55.297

55.815

56.316

56.800

57.270

57.724

58.164

58.590

59.003

59.462

59.790

60.165

60.529

60.882

61.224
( G-H298)/T

CAL/MOL.K

$-45.424$

$-45.425$

$-45.988$

- 46.404

$-46.824$

$-47.236$

$-47.635$

$-48.021$

$-48.396$

$-48.759$

$-49.112$

$-49.457$

$-49.792$

$-50.120$

$-50.440$

$-50.752$

$-51.058$

$-51 \cdot 357$

$-51.649$

$-51.936$

$-52.216$

$-52.490$

$-52.759$

$-53.022$

$-53.279$

$-53.532$

$-53.779$

$-54.022$ 
$\mathrm{T}$

$\begin{array}{cl}C P & H-H(\theta) \\ C A L / M O L \cdot K & C A L / M O L\end{array}$

$\begin{array}{lr}298 & 7.473 \\ 300 & 7.481 \\ 400 & 7.864 \\ 500 & 8.150 \\ 600 & 8.353 \\ 700 & 8.501 \\ 800 & 8.621 \\ 900 & 8.732 \\ 1000 & 8.845 \\ 1100 & 8.966 \\ 1200 & 9.099 \\ 1300 & 9.243 \\ 1400 & 9.395 \\ 1500 & 9.552 \\ 1600 & 9.712 \\ 1700 & 9.873 \\ 1800 & 10.031 \\ 1900 & 10.187 \\ 2000 & 10.339 \\ 2100 & 10.487 \\ 2200 & 10.630 \\ 2300 & 10.767 \\ 2400 & 10.901 \\ 2500 & 11.029\end{array}$

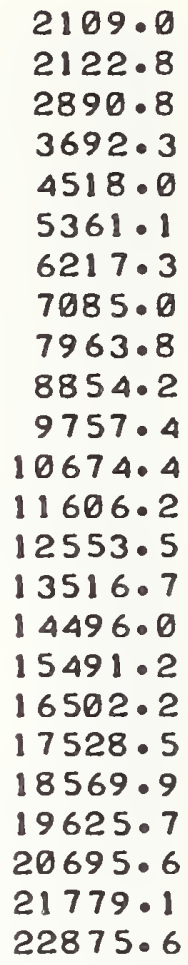

$\stackrel{S}{C A L / M O L \cdot K}$

$57 \cdot 351$

57.397

59.604

61.391

62.896

64.195

65.339

66.361

67.286

$68 \cdot 135$

$68 \cdot 921$

69.655

78.345

70.999

$71 \cdot 620$

$72 \cdot 214$

$72 \cdot 782$

$73 \cdot 329$

73.855

74.363

74.855

$75 \cdot 330$

$75 \cdot 791$

$76 \cdot 239$
( G-H298) /T

$C A L / M O L \cdot K$

$-57.351$

$-57.351$

$-57.649$

$-58.225$

$-58.881$

$-59.550$

$-60.203$

$-60.832$

$-61.431$

$-62.003$

$-62.547$

$-63.066$

$-63.561$

$-64.035$

$-64.490$

$-64.927$

$-65 \cdot 348$

$-65.754$

$-66 \cdot 146$

$-66.525$

$-66.892$

$-67.249$

$-67.595$

$-67.932$

MOLECULAR WEIGHT $=248.04$

MOLECULAR CONSTANTS ARE LISTED AS:

$L E V E L, W E, X E W E, Y E W E, B E, A L P H A E, D E, M U L T I P L I C I T Y, V O 0$

$\begin{array}{llllllllll}1 & 895.77 & 2.39 & 0 & .33264 & 1.3020 E-3 & 1.8330 E-7 & 1 & 0 \\ 2 & 864.1 & 2.31 & 0 & .32641 & .00123 & 1.8630 E-7 & 2 & 5305 \\ 3 & 846.4 & 2.4 & 0 & .32304 & 1.2940 E-3 & 1.3660 E-7 & 1 & 10601 . \\ 4 & 842.8 & 2.18 & 0 & .32497 & 1.2990 E-3 & 1.9400 E-7 & 2 & 11129 . \\ 5 & 835.1 & 2.39 & 0 & .32246 & .00128 & 1.9310 E-7 & 2 & 14490 \\ 6 & 839.2 & 2.5 & 0 & .32155 & .0013 & 1.8500 E-7 & 2 & 15946 . \\ 7 & 829.26 & 2.3 & 0 & .32309 & 1.3030 E-3 & 1.9900 E-7 & 1 & 16320 . \\ 8 & 816.2 & 2.26 & 0 & .31817 & .00124 & 1.9300 E-7 & 2 & 17998 \\ 9 & 816.2 & 2.4 & 0 & .3214 & .00124 & 2.0420 E-7 & 1 & 18338 .\end{array}$


$\mathrm{ThO}_{2}$ (c)

T

$\begin{array}{llr}298 & 14.77 & 0 . \\ 300 & 14.81 & 27 . \\ 400 & 16.21 & 1587 . \\ 500 & 16.96 & 3248 . \\ 600 & 17.44 & 4970 . \\ 700 & 17.81 & 6730^{\circ} \\ 800 & 18.11 & 8530 . \\ 900 & 18.37 & 10354 . \\ 1000 & 18.61 & 12204 . \\ 1100 & 18.84 & 14077 . \\ 1200 & 19.05 & 15971 . \\ 1300 & 19.26 & 17887 . \\ 1400 & 19.46 & 19823 . \\ 1500 & 19.66 & 21779 . \\ 1600 & 19.85 & 23754 . \\ 1700 & 20.04 & 25749 . \\ 1800 & 20.23 & 27763 . \\ 1900 & 20.42 & 29796 . \\ 2000 & 20.61 & 31847 . \\ 2100 & 20.80 & 33917 . \\ 2200 & 20.98 & 36006 . \\ 2300 & 21.17 & 38114 . \\ 2400 & 21.35 & 48239 . \\ 2500 & 21.53 & 42384 . \\ 2600 & 21.72 & 44546 . \\ 2700 & 21.90 & 46727 . \\ 2800 & 22.08 & 48927 . \\ & & \end{array}$

H-H298

CAL/MOL

$C P$
$C A L / M O L \cdot K$

.

7.

587.

48 .

6733

$6733^{\circ}$

10354 .

12204 .

14877 .

15971 .

17887.

19823.

21779 .

$25749^{\circ}$

27763 .

29796 .

31847 .

33917 .

36006 .

38114 .

48239 .

42384 .

44546 .

48927 .
$\stackrel{S}{C A L / M O L \cdot K}$

( G-H298)/T

CAL/MOL.K

$-15.59$

$-15.59$

$-16 \cdot 19$

$-17.37$

$-18.72$

$-20.10$

$-21 \cdot 45$

$-22.76$

$-24.61$

$-25.20$

$-26.34$

$-27.42$

$-28 \cdot 45$

$-29 \cdot 44$

$-30.39$

$-31 \cdot 30$

$-32.17$

$-33.82$

$-33.83$

$-34.61$

$-35.37$

$-36 \cdot 10$

$-36.81$

$-37.49$

$-38.16$

$-38.81$

$-39.45$ 
I

$\begin{array}{ll}298 & 11.317 \\ 300 & 11.334 \\ 400 & 12.110 \\ 500 & 12.621 \\ 600 & 12.955 \\ 700 & 13.180 \\ 800 & 13.336 \\ 900 & 13.448 \\ 1000 & 13.531 \\ 1100 & 13.593 \\ 1200 & 13.642 \\ 1300 & 13.680 \\ 1400 & 13.711 \\ 1500 & 13.736 \\ 1600 & 13.757 \\ 1700 & 13.774 \\ 1800 & 13.788 \\ 1900 & 13.801 \\ 2800 & 13.811 \\ 2100 & 13.820 \\ 2200 & 13.828 \\ 2300 & 13.835 \\ 2400 & 13.841 \\ 2500 & 13.846 \\ 2600 & 13.851 \\ 2700 & 13.855 \\ 2800 & 13.859 \\ 2900 & 13.863 \\ 3000 & 13.866\end{array}$

$H-H(\theta)$

CAL/MOL

CAL/MOL.K

$2921 \cdot 2$

$2942 \cdot 2$

4116.9

5355.3

6635.3

7942.7

9268.9

10608.4

11957.5

13313.9

14675.7

16041.9

17411.5

18783.9

$20158 \cdot 6$

21535.1

22913.2

24292.7

25673.3

27854.8

28437.3

29820.4

31204.2

$32588 \cdot 6$

33973.5

35358.8

36744.6

38130.6

39517.1
$\stackrel{S}{C A L / M O L \cdot K}$

68.701

68.771

$72 \cdot 145$

74.906

77.239

79.254

81.025

82.602

84.024

85.316

86.501

87.595

88.610

89.557

90.444

91.278

92.066

92.812

93.520

94.194

94.837

95.452

96.041

96.606

97.149

97.672

98.176

98.662

99.132
$(G-H 298) / T$

CAL/MOL.K

$-68.701$

$-68.701$

$-69.156$

$-70.038$

$-71.049$

$-72.080$

$-73.098$

$-74.061$

$-74.987$

$-75.868$

$-76.706$

$-77.502$

$-78.260$

$-78.981$

$-79.670$

$-80.329$

$-80.959$

$-81.564$

$-82 \cdot 144$

$-82 \cdot 702$

$-83.239$

$-83.757$

$-84.256$

$-84.739$

$-85.206$

$-85.658$

$-86.096$

$-86.521$

$-86.934$

FREQ 787 CM-1 MULT 1

FREQ $120 \mathrm{CM}-1$ MULT 1

FREQ 735 CM-1 MULT 1

MOLECULAR WEIGHT 264.037

SYMMETRY 2

MOMENTS $23.486 \quad 88.795 \quad 112.281$ IN AMU A2 GROUND STATE DEGENERACY 1 
T

298
300
400
500
600
700
800
900
1000
1100
1200
1300
1400
1500
1600
1700
1800
1900
2000
2100
2200
2300
2400
2500

$\mathrm{CP}$
$C A L / M O L \cdot K$

$$
\begin{aligned}
& 8.285 \\
& 8.292 \\
& 8.573 \\
& 8.735 \\
& 8.839 \\
& 8.912 \\
& 8.968 \\
& 9.014 \\
& 9.054 \\
& 9.090 \\
& 9.123 \\
& 9.155 \\
& 9.185 \\
& 9.215 \\
& 9.244 \\
& 9.273 \\
& 9.302 \\
& 9.331 \\
& 9.361 \\
& 9.390 \\
& 9.420 \\
& 9.450 \\
& 9.481 \\
& 9.512
\end{aligned}
$$

$H-H(\theta)$

CAL/MOL

2226.8
2242.1
3086.7
3952.8
4831.8
5719.5
6613.7
7512.9
8416.3
9323.6
10234.2
1114.1
12065.1
12985.1
13908.0
14833.8
15762.6
16694.2
17628.8
18566.4
19506.9
20450.4
21397.0
22346.6

$\stackrel{S}{C A L / M O L \cdot K}$

$61.47 i$

$61 \cdot 523$

63.951

65.882

67.485

68.853

70.047

71.165

72.057

72.922

73.714

74.445

$75 \cdot 124$

75.758

76.353

76.914

77.445

77.948

78.426

78.883

79.320

79.738

80.140

80.527
$(G-H 298) / T$

CAL/MOL.K

-61.471
-61.472
-61.801
-62.430
-63.143
-63.863
-64.563
-65.232
-65.868
-66.470
-67.041
-67.582
-68.097
-68.586
-69.053
-69.498
-69.925
-70.333
-70.725
-71.102
-71.465
-71.815
-72.153
-72.479

MOLECULAR WEIGHT $=251.04$

ALPHAE APPROXIMATED AS $2.0386 E-3$ STATE 1

DE APPROXIMATED AS $4(B E)+3 /(W E)+21.9931 E-7$ STATE 1

MOLECULAR CONSTANTS ARE LISTED AS

LEVEL, WE, XEWE, YEWE, BE, ALPHAE, DE, MULTIPLICI TY, VØO

$148940.2284 \quad 2.0386 E-3 \quad 1.9931 E-7 \quad 4 \quad 0$ 
$\mathrm{ThF}_{2}(\mathrm{~g})$

T

$\begin{array}{cl}C P & H-H(B) \\ C A L / M O L \cdot K & C A L / M O L\end{array}$

3111.7

3134.9

4418.0

$5739 \cdot 7$

7082.9

8439.0

9803.5

11173.7

12548.0

13925.3

15304.8

16686.1

$18068 \cdot 7$

19452.5

20837.1

$22222 \cdot 5$

$23608 \cdot 5$

24995.1

26382.0

27769.4

$29157 \cdot 1$

30545.1

31933.3

$33321 \cdot 8$
$S$

CAL/MOL.K

70.533

70.611

74.299

77.247

79.695

81.785

83.687

85.221

86.669

87.982

89.182

90.288

91.312

92.267

93.161

94.001

94.793

95.542

96.254

96.931

97.576

98.193

$98 \cdot 784$

99.351
$(\mathrm{G}-\mathrm{H} 298) / T$

CAL/MOL - K

$-70.533$

$-70.534$

$-71.033$

$-71.991$

$-73.077$

$-74.175$

$-75.243$

$-76.263$

$-77.233$

$-78 \cdot 151$

$-79.821$

$-79.846$

$-80.629$

$-81.373$

$-82.882$

$-82.759$

$-83.406$

$-84.025$

$-84.619$

$-85.189$

$-85.737$

$-86.266$

$-86.775$

$-87.267$

FREQ 447 CM-1 MULT 1

FREQ $130 \mathrm{CM}-1$ MULT 1

FREQ 483 CM-1 MULT 1

MOLECULAR WEIGHT 270.04

SYMMETRY 2

MOMENTS $137.64 \quad 25.717 \quad 163.36$ IN AMU A2 GROUND STATE DEGENERACY 1 
$\mathrm{ThF}_{3}(\mathrm{~g})$

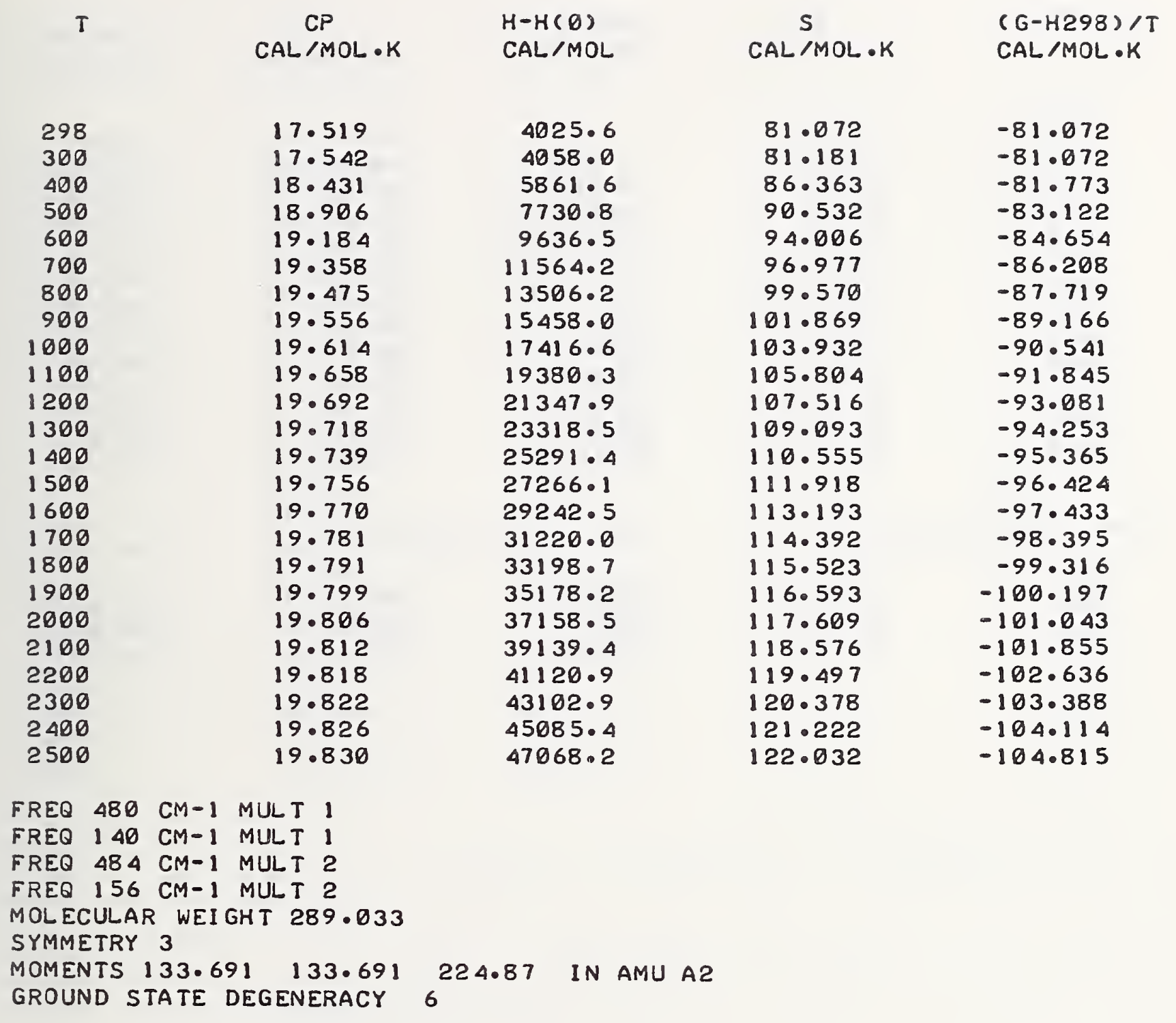


$\mathrm{ThF}_{4}(\mathrm{c}, \mathrm{l})$

\begin{tabular}{|c|c|c|c|c|}
\hline$T$ & $\begin{array}{c}C P \\
C A L / M O L \cdot K\end{array}$ & $\begin{array}{l}\text { H-H298 } \\
\text { CAL/MOL }\end{array}$ & $\stackrel{S}{\mathrm{CAL} / \mathrm{MOL} \cdot \mathrm{K}}$ & $\begin{array}{l}(G-H 298) / T \\
C A L / M O L \cdot K\end{array}$ \\
\hline 298 & $26 \cdot 42$ & g. & 33.95 & -33.95 \\
\hline 300 & $26 \cdot 47$ & 49. & 34.11 & -33.95 \\
\hline 400 & $28 \cdot 13$ & 2789. & 41.98 & -35.81 \\
\hline 500 & 29.00 & 5649. & $48 \cdot 36$ & -37.87 \\
\hline 600 & 29.57 & 8579. & 53.78 & $-39 \cdot 41$ \\
\hline 700 & 29.99 & 11557 & $58 \cdot 29$ & $-41 \cdot 78$ \\
\hline 800 & 30.33 & 14574. & $62 \cdot 32$ & -44.10 \\
\hline 980 & 30.63 & 17622 & 65.91 & -46.33 \\
\hline 1000 & 30.90 & $20699^{\circ}$ & 69.15 & $-48 \cdot 45$ \\
\hline 1100 & 31.15 & 23802 & 72.11 & $-50 \cdot 47$ \\
\hline 1200 & 31.39 & 26929. & 74.83 & $-52 \cdot 39$ \\
\hline 1300 & $31 \cdot 62$ & 30080 & 77.35 & -54.21 \\
\hline 1383 & $31 \cdot 81$ & 32712. & $79 \cdot 32$ & -55.66 \\
\hline$=10000$ & CAL/MOL & $S T=7.2307$ & $C A L / M O L \cdot K$ & AT $1383 \mathrm{~K}$ \\
\hline 1383 & 32.60 & 42712 & 86.55 & -55.66 \\
\hline 1400 & 32.00 & 43256 & 86.94 & -56.04 \\
\hline 1500 & 32.00 & 46456 & 89.14 & $-58 \cdot 17$ \\
\hline
\end{tabular}


$\mathrm{ThF}_{4}$ (g)

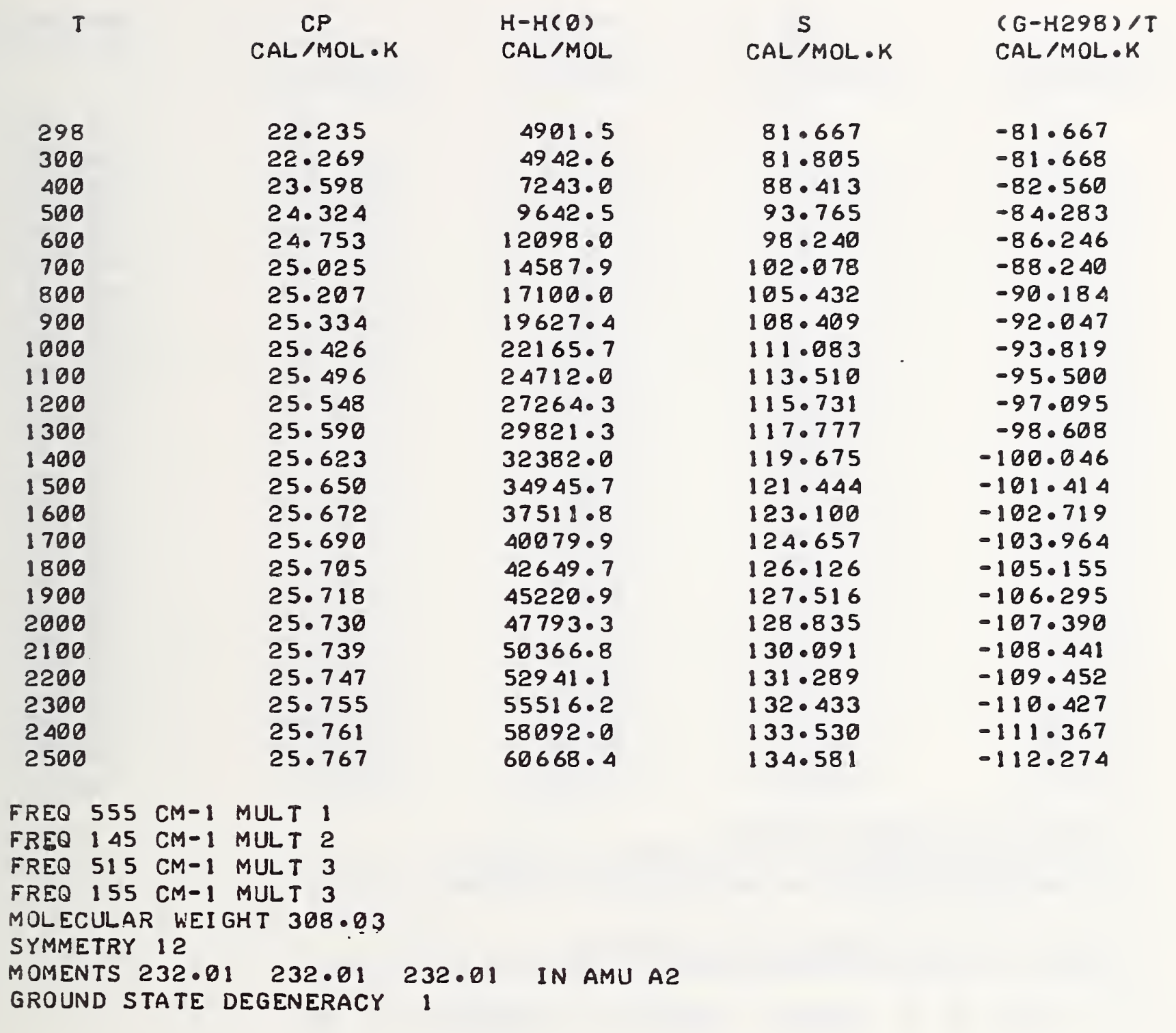


T

$\stackrel{C P}{C A L / M O L \cdot K}$

$$
\begin{aligned}
& 8.714 \\
& 8.718 \\
& 8.865 \\
& 8.951 \\
& 9.012 \\
& 9.061 \\
& 9.104 \\
& 9.144 \\
& 9.183 \\
& 9.220 \\
& 9.258 \\
& 9.295 \\
& 9.333 \\
& 9.372 \\
& 9.411 \\
& 9.451 \\
& 9.492 \\
& 9.534 \\
& 9.577 \\
& 9.620 \\
& 9.665 \\
& 9.710 \\
& 9.757 \\
& 9.804
\end{aligned}
$$

$H-H(\theta)$

CAL/MOL

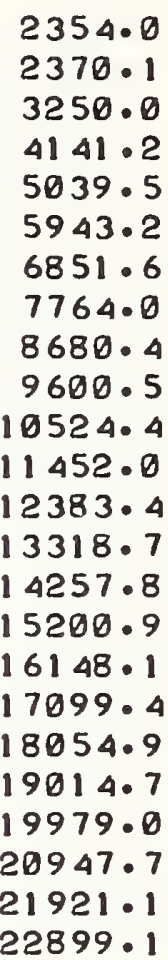

$\stackrel{S}{C A L / M O L \cdot K}$

64.325

64.379

66.910

68.898

70.535

$71 \cdot 928$

$73 \cdot 140$

74.215

$75 \cdot 180$

76.056

76.859

77.601

$78 \cdot 290$

78.935

79.540

80.110

80.651

$81 \cdot 164$

81.653

82.119

82.566

82.995

83.408

83.805
( G-H298) /T

CAL $/ M O L \cdot K$

$-64.325$

$-64.325$

$-64.669$

$-65.323$

$-66.059$

$-66.800$

$-67.518$

$-68 \cdot 203$

$-68.853$

.69 .468

$-70.051$

$-70.682$

$-71 \cdot 126$

$-71.625$

$-72.100$

$-72 \cdot 553$

$-72.987$

$-73.483$

$-73 \cdot 802$

$-74.186$

$-74.555$

$-74.911$

$-75.255$

$-75.587$

MOLECULAR WEIGHT $=267.49$

ALPHAE APPROXIMATED AS $6.0992 E-4$ STATE 1

DE APPROXIMATED AS $4(B E)+3 /(W E): 23.3206 E-8$ STATE 1

MOLECULAR CONSTANTS ARE LISTED AS

LEVEL, WE, XEWE, YEWE, BE, ALPHAE, DE, MUL TIPLICI TY, VOO
129
20
.08912
$6.0992 E-4$
$3 \cdot 3206 E-8$
40 
$\mathrm{ThCl}_{2}(\mathrm{~g})$

$\begin{array}{rll}T & \begin{array}{c}\text { CP } \\ \text { CAL/MOL.K }\end{array} & \begin{array}{l}H-H(O) \\ \text { CAL/MOL }\end{array} \\ 298 & & \\ 300 & 13.212 & 3359.9 \\ 400 & 13.220 & 3384.3 \\ 500 & 13.504 & 4722.4 \\ 600 & 13.645 & 6080.5 \\ 700 & 13.724 & 7449.3 \\ 800 & 13.772 & 8824.3 \\ 900 & 13.804 & 10203.2 \\ 1000 & 13.826 & 11584.8 \\ 1100 & 13.842 & 12968.2 \\ 1200 & 13.854 & 14353.0 \\ 1300 & 13.863 & 15738.9 \\ 1400 & 13.870 & 17125.5 \\ 1500 & 13.875 & 18512.7 \\ 1600 & 13.880 & 19900.5 \\ 1700 & 13.883 & 21288.6 \\ 1800 & 13.886 & 22677.1 \\ 1900 & 13.889 & 24065.9 \\ 2000 & 13.891 & 25454.9 \\ 2100 & 13.893 & 26844.1 \\ 2200 & 13.895 & 28233.5 \\ 2300 & 13.896 & 29623.0 \\ 2400 & 13.897 & 31012.7 \\ 2500 & 13.898 & 32402.5 \\ & 13.899 & 33792.3\end{array}$

$S$ CAL $/ M O L \cdot K$

$(G-H 298) / T$

CAL/MOL $\cdot K$

75.766
75.848
79.695
82.725
85.220
87.340
89.181
90.808
92.266
93.586
94.792
95.901
96.929
97.887
98.783
99.625
100.418
101.169
101.882
102.560
103.206
103.824
104.415
104.983

$-75.766$

$-75.766$

$-76.289$

$-77.284$

$-78.405$

$-79.534$

$-80.627$

$-81 \cdot 669$

$-82.658$

$-83.592$

$-84.476$

$-85 \cdot 312$

$-86.106$

$-86.360$

$-87.577$

$-88.262$

$-88 \cdot 915$

$-89 \cdot 540$

$-90.140$

$-90.715$

$-91 \cdot 268$

$-91.801$

$-92 \cdot 314$

$-92 \cdot 810$

FREQ 388 CM-1 MULT I

FREQ 84 CM-1 MULT I

FREQ $315 \mathrm{CM}-1$ MULT 1

MOLECULAR WEIGHT 302.944

SYMMETRY 2

MOMENTS $372.8 \quad 62.09 \quad 434.89$ IN AMU A2

GROUND STATE DEGENERACY 1 
$\mathrm{ThCl}_{3}(\mathrm{~g})$

$T$

$\begin{array}{ll}298 & 18.643 \\ 300 & 18.656 \\ 400 & 19.155 \\ 500 & 19.403 \\ 600 & 19.542 \\ 700 & 19.628 \\ 800 & 19.634 \\ 900 & 19.723 \\ 1000 & 19.751 \\ 1100 & 19.772 \\ 1200 & 19.787 \\ 1300 & 19.800 \\ 1400 & 19.810 \\ 1500 & 19.818 \\ 1600 & 19.824 \\ 1700 & 19.830 \\ 1800 & 19.834 \\ 1900 & 19.838 \\ 2000 & 19.841 \\ 2100 & 19.844 \\ 2200 & 19.847 \\ 2300 & 19.849 \\ 2400 & 19.851 \\ 2500 & 19.852\end{array}$

$H-H(B)$

CAL/MOL

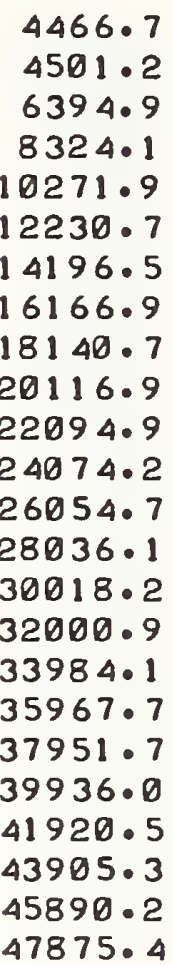

$\stackrel{S}{C A L / M O L} \cdot K$

$88 \cdot 331$

$88 \cdot 447$

93.891

98.195

101.746

104.765

107.390

109.711

111.790

113.674

115.395

116.979

118.447

119.814

121.093

122.295

123.429

124.501

125.519

126.487

127.410

128.292

$129 \cdot 137$

129.948
$(G-H 298) / T$

CAL/MOL.K

$-88 \cdot 331$

$-88 \cdot 332$

$-89.070$

$-90.480$

$-92.070$

$-93.674$

$-95 \cdot 228$

$-96.710$

$-98 \cdot 116$

$-99.446$

$-100.705$

$-101.896$

$-103.027$

$-104.101$

$-105.123$

$-106.098$

$-107.030$

$-107.922$

$-108.776$

$-109.597$

$-110.386$

$-111 \cdot 145$

$-111.877$

$-112.584$

FREQ 320 CM-1 MULT 1

FREQ 100 CM-1 MULT 1

FREQ 333 CM-1 MULT 2

FREQ 105 CM-1 MULT 2

MOLECULAR WEIGHT 338.397

SYMMETRY 3

MOMENTS $353.677 \quad 353.677 \quad 609.06$ IN AMU A2

GROUND STATE DEGENERACY 6 
$\mathrm{ThCl}_{4}(\mathrm{c}, 1)$

\begin{tabular}{|c|c|c|c|c|}
\hline$T$ & $\begin{array}{c}C P \\
C A L / M O L \cdot K\end{array}$ & $\begin{array}{l}\mathrm{H}-\mathrm{H} 298 \\
\mathrm{CAL} / \mathrm{MOL}\end{array}$ & $\stackrel{S}{C A L / M O L \cdot K}$ & $\begin{array}{l}(G-H 298) / T \\
C A L / M O L \cdot K\end{array}$ \\
\hline $\begin{array}{l}298 \\
380 \\
400 \\
500 \\
600 \\
679\end{array}$ & $\begin{array}{l}23.75 \\
28.78 \\
30.06 \\
30.94 \\
31.68 \\
32.21\end{array}$ & $\begin{array}{r}0 . \\
53 . \\
3000 \\
6052 \\
9184 . \\
11708 .\end{array}$ & $\begin{array}{l}45.50 \\
45.68 \\
54.15 \\
60.95 \\
66.66 \\
70.61\end{array}$ & $\begin{array}{l}-45.50 \\
-45.50 \\
-46.65 \\
-48.85 \\
-51.36 \\
-53.37\end{array}$ \\
\hline$H T=1200$ & CAL/MOL & $S T=1.7673$ & CAL/MOL.K & AT $679 \mathrm{~K}$ \\
\hline $\begin{array}{r}679 \\
700 \\
800 \\
900 \\
1000 \\
1042\end{array}$ & $\begin{array}{l}32.21 \\
32.34 \\
32.97 \\
33.57 \\
34.16 \\
34.41\end{array}$ & $\begin{array}{l}12908^{\circ} \\
13585^{\circ} \\
16851^{\circ} \\
20179^{\circ} \\
23565^{\circ} \\
250060^{\circ}\end{array}$ & $\begin{array}{l}72 \cdot 38 \\
73.36 \\
77.72 \\
81.64 \\
85.21 \\
86.62\end{array}$ & $\begin{array}{l}-53.37 \\
-53.96 \\
-56.66 \\
-59.22 \\
-61.64 \\
-62.62\end{array}$ \\
\hline$H I=14690$ & CAL/MOL & $S T=14.098$ & $\mathrm{CAL} / \mathrm{MOL} \cdot \mathrm{K}$ & AT $1042 \mathrm{~K}$ \\
\hline $\begin{array}{l}1042 \\
1100 \\
1200 \\
1300 \\
1400 \\
1500\end{array}$ & $\begin{array}{l}40.0 \theta \\
4 \theta .00 \\
4 \theta .0 \theta \\
40.00 \\
4 \theta .00 \\
40.00\end{array}$ & $\begin{array}{l}39696 . \\
42016 . \\
46016 . \\
50016 . \\
54016 . \\
58016 .\end{array}$ & $\begin{array}{l}100.72 \\
102.88 \\
106.36 \\
109.57 \\
112.53 \\
115.29\end{array}$ & $\begin{array}{l}-62.62 \\
-64.69 \\
-68.02 \\
-71.09 \\
-73.95 \\
-76.61\end{array}$ \\
\hline
\end{tabular}


$\mathrm{ThCl}_{4}(\mathrm{~g})$

T

$\begin{array}{cl}\text { CP } & H-H(B) \\ C A L / M O L \cdot K & C A L / M O L\end{array}$

24.273

24.290

24.922

25.237

25.414

25.523

25.594

25.644

25.679

25.706

25.726

25.742

25.754

25.764

25.773

25.780

25.785

25. 790

25.794

25.798

25.801

25.804

25.806

25.808

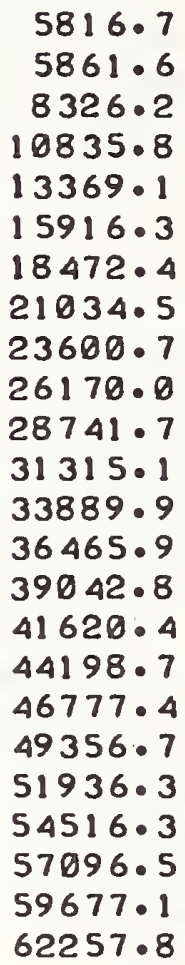

$\stackrel{S}{C A L / M O L} \cdot K$

95.000

95.150

102.236

107.835

112.453

116.380

119.793

122.810

125.514

127.963

130.201

132.260

134.169

135.946

137.609

139.172

140.645

142.039

143.362

144.621

145.821

146.968

148.067

149.120
(G-H298)/T

CAL/MOL.K

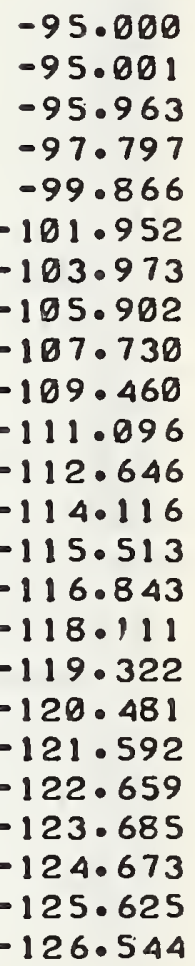

$-95.000$

$-95.001$

$-95.963$

$-97.797$

$-99.866$

$-101.952$

$-103.973$

$-195.902$

$-107.730$

$-109.460$

$-111.096$

$-112.646$

$-114.116$

$-115.513$

$-116.843$

$-118.111$

$-119.322$

$-120.481$

$-121.592$

$-122.659$

$-123.685$

$-124.673$

$-126 \cdot 544$

FREQ 300 CM-1 MULT 1

FREQ $70 \mathrm{CM}-1$ MULT 2

FREQ $335 \mathrm{CM}-1$ MULT 3

FREQ 75 CM-1 MULT 3

MOLECULAR WEIGHT 373.85

SYMMETRY 12

MOMENTS 629.31629 .31629 .31 IN AMU A2

GROUND STATE DEGENERACY 1 
T

$\begin{array}{ll}298 & 8.952 \\ 300 & 8.955 \\ 400 & 9.072 \\ 500 & 9.164 \\ 600 & 9.249 \\ 700 & 9.332 \\ 800 & 9.416 \\ 900 & 9.504 \\ 1000 & 9.595 \\ 1100 & 9.691 \\ 1200 & 9.790 \\ 1300 & 9.894 \\ 1400 & 10.803 \\ 1500 & 10.116 \\ 1600 & 10.234 \\ 1700 & 10.357 \\ 1800 & 10.485 \\ 1900 & 10.617 \\ 2000 & 10.754 \\ 2100 & 10.895 \\ 2200 & 11.042 \\ 2300 & 11.193 \\ 2400 & 11.349 \\ 2500 & 11.510\end{array}$

$\mathrm{H}-\mathrm{H}(\theta)$

$C A L / M O L$

$2452 \cdot 9$

2469.5

$3371 \cdot 2$

4283.1

5203.8

$6132 \cdot 8$

7070.2

8016.2

$8971 \cdot 1$

9935.4

10909.4

11893.6

$12888 \cdot 4$

13894.4

14911.9

15941.4

16983.5

18038.5

19107.0

20189.4

21286.3

22398.0

23525.1

24668.0
$\stackrel{S}{C A L / M O L} \cdot K$

$67 \cdot 153$

67.209

69.801

71.835

73.513

74.943

76.193

77.305

78.308

79.224

80.069

80.852

81.585

82.274

82.926

83.544

84.133

84.696

85.237

85.757

86.258

86.743

87.212

87.668
(G-H298) /T

CAL/MOL.K

$-67.153$

$-67.153$

$-67.506$

$-68 \cdot 175$

$-68.928$

$-69.686$

$-70.421$

$-71 \cdot 124$

$-71.790$

$-72.422$

$-73.021$

$-73.590$

$-74.131$

$-74.647$

$-75.139$

$-75.609$

$-76.060$

$-76.493$

$-76.910$

$-77.311$

$-77.697$

$-78.071$

$-78 \cdot 432$

$-78 \cdot 782$

MOLECULAR WEIGHT $=311.94$

ALPHAE APPROXIMATED AS $3.0458 E-4$ STATE 1

DE APPROXIMATED AS $4(B E)+3 /(W E) \div 27.0029 E-9$ STATE 1

MOLECULAR CONSTANTS ARE LISTED AS:

LEVEL, WE, XEWE, YEWE, BE, ALPHAE, DE, MUL TIPLICI TY, VOO

$119420.040393 .0458 E-4 \quad 7.0029 E-9 \quad 4 \quad 0$ 
ThBr, ( T $_{2}$

\section{$T$}

$\begin{array}{cl}C P & H-H(\theta) \\ C A L / M O L \cdot K & C A L / M O L\end{array}$

$\begin{array}{lll}298 & 13.559 & 3556.7 \\ 300 & 13.563 & 3581.8 \\ 400 & 13.711 & 4946.5 \\ 500 & 13.781 & 6321.4 \\ 600 & 13.820 & 7701.6 \\ 700 & 13.844 & 9084.9 \\ 800 & 13.859 & 10470.1 \\ 900 & 13.870 & 11856.6 \\ 1000 & 13.877 & 13244.0 \\ 1100 & 13.883 & 14632.0 \\ 1200 & 13.887 & 16020.6 \\ 1300 & 13.891 & 17409.5 \\ 1400 & 13.893 & 18798.7 \\ 1500 & 13.896 & 20188.1 \\ 1600 & 13.897 & 21577.8 \\ 1700 & 13.899 & 22967.6 \\ 1800 & 13.900 & 24357.6 \\ 1900 & 13.901 & 25747.6 \\ 2000 & 13.902 & 27137.8 \\ 2100 & 13.903 & 28528.0 \\ 2200 & 13.903 & 29918.3 \\ 2300 & 13.904 & 31308.7 \\ 2400 & 13.904 & 32699.1 \\ 2500 & 13.905 & 34089.6\end{array}$

$S$

CAL/MOL $\cdot K$
$(G-H 298) / T$

CAL/MOL.K
$-81 \cdot 009$

$-81.009$

$-81 \cdot 543$

$-82.556$

$-83.693$

$-84.837$

$-85.942$

$-86.995$

$-87.991$

$-88.933$

$-89.823$

$-90.665$

$-91.464$

$-92.222$

$-92.943$

$-93.631$

$-94.287$

$-94.915$

$-95.517$

$-96.095$

$-96.650$

$-97.185$

$-97.700$

$-98 \cdot 197$

FREQ 196 CM-1 MULT 1

FREO 61 CM-1 MULT 1

FREO 232 CM-1 MULT 1

MULECULAR WEIGHT 391.856

SYMMETRY 2

MOMENTS $123.221 \quad 956.989 \quad 1080.21$ IN AMU A2

GROUND STATE DEGENERACY 1 


\section{$\mathrm{ThBr}_{3}(\mathrm{~g})$}

T

$\begin{array}{ll}298 & 19.311 \\ 300 & 19.317 \\ 400 & 19.553 \\ 500 & 19.666 \\ 600 & 19.728 \\ 700 & 19.766 \\ 800 & 19.790 \\ 900 & 19.807 \\ 1000 & 19.820 \\ 1100 & 19.829 \\ 1200 & 19.835 \\ 1300 & 19.841 \\ 1400 & 19.845 \\ 1500 & 19.848 \\ 1600 & 19.851 \\ 1700 & 19.854 \\ 1800 & 19.856 \\ 1900 & 19.857 \\ 2000 & 19.859 \\ 2100 & 19.860 \\ 2200 & 19.861 \\ 2300 & 19.862 \\ 2400 & 19.863 \\ 2500 & 19.863\end{array}$

$H-H(O)$

CAL/MOL

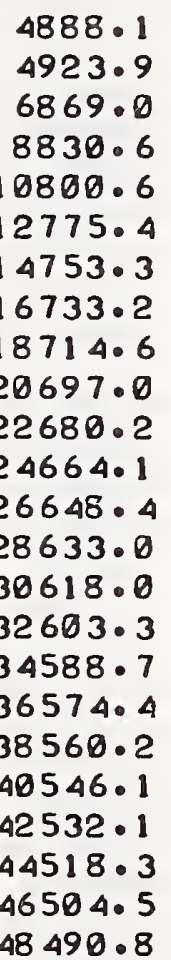

$S$

CAL/MOL.K

$96 \cdot 829$
$96 \cdot 949$
$102 \cdot 543$
$106 \cdot 920$
$110 \cdot 511$
$113 \cdot 555$
$116 \cdot 196$
$118 \cdot 528$
120.616
$122 \cdot 505$
124.231
$125 \cdot 819$
$127 \cdot 289$
$128 \cdot 659$
129.940
131.143
$132 \cdot 278$
$133 \cdot 352$
134.370
$135 \cdot 339$
$136 \cdot 263$
$137 \cdot 146$
137.991
138.802

$(\mathrm{G}-\mathrm{H} 298) / T$ CAL/MOL.K

$-96.829$

$-96.830$

$-97.591$

$-99.835$

$-100.657$

$-102.288$

$-103.865$

$-105.367$

$-106.789$

$-108.134$

$-109.404$

$-110.607$

$-111.746$

$-112.829$

$-113.859$

$-114.840$

$-115.778$

$-116.675$

$-117.534$

$-118.359$

$-119.152$

$-119.915$

$-120.651$

$-121.361$

FREQ 200 CM-1 MULT 1

FREQ 68 CM-1 MULT 1

FREQ $223 \mathrm{CM}-1$ MULT 2

FREQ 66 CM-1 MULT 2

MOLECULAR WEIGHT 471.765

SYMMETRY 3

MOMENTS $872.26 \quad 872.26 \quad 1563.51$ IN AMU A2

GROUND STATE DEGENERACY 6 
$\mathrm{ThBr}_{4}(\mathrm{c}, \mathrm{I})$

\begin{tabular}{|c|c|c|c|c|c|}
\hline \multicolumn{2}{|c|}{$T$} & $\begin{array}{c}C P \\
C A L / M O L \cdot K\end{array}$ & $\begin{array}{l}H-H 298 \\
C A L / M O L\end{array}$ & $\stackrel{S}{C A L / M O L \cdot K}$ & $\begin{array}{l}(G-H 298) / T \\
C A L / M D L \cdot K\end{array}$ \\
\hline & & 29.92 & 0 . & 54.50 & -54.50 \\
\hline & & 29.95 & 55. & 54.69 & -54.50 \\
\hline & & 31.02 & 3109. & 63.46 & -55.69 \\
\hline & & 31.71 & 6247. & 70.46 & -57.97 \\
\hline & & $32 \cdot 25$ & 9446. & 76.29 & $-60 \cdot 55$ \\
\hline & & 32.69 & 12466 & 80.97 & $-62 \cdot 98$ \\
\hline$H T=$ & 1000 & $C A L / M O L$ & \multicolumn{2}{|c|}{$S T=1.443 \mathrm{CAL} / \mathrm{MOL} \cdot \mathrm{K}$} & AT $693 \mathrm{~K}$ \\
\hline & & 32.69 & 13466 & $82 \cdot 42$ & $-62 \cdot 98$ \\
\hline & & 32.72 & 13695 & 82.74 & -63.18 \\
\hline & & 33.15 & $16989^{\circ}$ & 87.14 & -65.91 \\
\hline & & 33.56 & 20325 & 91.07 & $-68 \cdot 49$ \\
\hline & & 33.77 & 22075 & 92.96 & $-69 \cdot 77$ \\
\hline \multicolumn{2}{|r|}{7000} & $C A L / M O L$ & $S T=7.3529 \mathrm{C}$ & $C A L / M O L \cdot K$ & AT $952 \mathrm{~K}$ \\
\hline & & 41.00 & 29075 & $100 \cdot 31$ & $-69 \cdot 77$ \\
\hline & & 41.00 & 31043 & 102.33 & $-71 \cdot 29$ \\
\hline & & 41.00 & 35143. & 106.24 & -74.29 \\
\hline & & 41.00 & 39243. & 109.31 & -77.10 \\
\hline & & 41.90 & 43343. & 113.09 & -79.75 \\
\hline & & 41.90 & $47 \Delta 43$. & 116.13 & -82.24 \\
\hline & & 41.00 & 51543. & 118.95 & -84.59 \\
\hline
\end{tabular}


$\mathrm{ThBr}_{4}(\mathrm{~g})$

T

$\begin{array}{ll}298 & 25.064 \\ 300 & 25.073 \\ 400 & 25.396 \\ 500 & 25.551 \\ 600 & 25.636 \\ 700 & 25.688 \\ 800 & 25.722 \\ 900 & 25.745 \\ 1000 & 25.762 \\ 1100 & 25.774 \\ 1200 & 25.783 \\ 1300 & 25.791 \\ 1400 & 25.797 \\ 1500 & 25.801 \\ 1600 & 25.805 \\ 1700 & 25.808 \\ 1800 & 25.811 \\ 1900 & 25.813 \\ 2000 & 25.815 \\ 2100 & 25.817 \\ 2200 & 25.818 \\ 2300 & 25.820 \\ 2400 & 25.821 \\ 2500 & 25.822\end{array}$

$H-H(D)$

CAL/MOL

$6228 \cdot 0$

6274.4

8800.0

11348.2

13908.0

16474.4

19045.0

21618.4

24193.7

26770.6

29348.5

31927.2

34506.6

37086.5

39666.8

42247.5

$44828 \cdot 5$

47409.7

49991.1

52572.7

55154.5

57736.4

$50318 \cdot 4$

62900.6
$S$

$C A L / M O L \cdot K$

102.720

162.875

110.139

115.824

120.491

124.447

127.879

130.910

133.624

136.080

138.323

140.387

142.298

144.678

145.744

147.308

148.783

150.179

151.503

152.763

153.964

155.111

156.210

157.264
( G-H298) /T

CAL/MOL.K

$-102 \cdot 720$

$-102.720$

$-103.709$

$-105.584$

$-107.691$

$-109.809$

$-111.858$

$-113.810$

$-115.658$

$-117.405$

$-119.056$

$-120.618$

$-122.099$

$-123.506$

$-124.844$

$-126.120$

$-127.339$

$-128.504$

$-129.622$

$-130.694$

$-131.724$

$-132.716$

$-133.673$

$-134.595$

FREQ 205 CM-1 MULT 1

FREQ 58 CM-1 MULT 2

FREQ $220 \mathrm{CM}-1$ MULT 3

FREQ 72 CM-1 MULT 3

MOLECULAR WEIGHT 551.654

SYMMETRY 12

MOMENTS $1576.4 \quad 1576.4 \quad 1576.4$ IN AMU A2 GROUND STATE DEGENERACY 1 
T

$C P$
$C A L / M O L \cdot K$

8.980

8.982

9.066

9.135

9.199

9.262

9.327

9.394

9.464

9.536

9.611

9.690

9.772

9.856

9.944

10.036

10.130

10.228

10.329

10.433

10.541

10.651

10.765

10.883
$\mathrm{H}-\mathrm{H}(\theta)$

CAL/MOL

2494.2

2510.8

3413.4

4323.5

5240.2

6163.2

7092.7

8028.7

8971.6

9921.5

10878.9

11843.9

12817.0

13798.3

14788.3

15787.3

16795.5

17813.4

18841.2

19879.3

20927.9

21987.5

$23058 \cdot 3$

24140.7
$\stackrel{S}{C A L / M O L \cdot K}$

68.950

69.006

71.602

73.632

75.302

76.724

77.963

79.064

80.056

80.959

81.789

82.559

83.277

83.951

84.586

85.188

85.760

86.305

86.827

87.328

87.810

88.275

88.724

89.158
(G-H298)/T

CAL/MOL.K

$-68 \cdot 950$

$-68.950$

$-69.303$

$-69.973$

$-70.725$

$-71 \cdot 482$

$-72.215$

$-72.915$

$-73.578$

$-74.207$

$-74.802$

$-75.367$

$-75.904$

$-76.415$

$-76.902$

$-77.368$

$-77.814$

$-78.242$

$-78.654$

$-79.050$

$-79.431$

$-79.799$

$-80.155$

$-80.500$

MOLECULAR WEIGHT $=358.94$

ALPHAE APPROXIMATED AS $1.3447 E-4$ STATE 1

DE APPROXIMATED AS $4(B E): 3 /(W E): 22.8410 E-9$ STATE 1

MOLECULAR CONSTANTS ARE LISTED AS:

LEVEL, WE, XEWE, YEWE, BE, ALPHAE, DE, MULTIPLI CI TY, VOO

$115110.0253 \quad 1.3447 E-4 \quad 2.8410 E-9 \quad 4 \quad 0$ 
$\operatorname{ThI}_{2}(\mathrm{~g})$

\section{T}

CP
$C A L / M O L \cdot K$

13. 713

13.716

13.799

13.839

13.860

13.874

13.882

13.888

13.892

13.895

13.898

13.900

13.901

13.902

13.903

13.904

13.905

13.905

13.906

13.906

13.906

13.907

13.907

13.907
$H-H(\theta)$

CAL/MOL

$$
3691 \cdot 1
$$

3716.5

5092.8

6475.0

7860.0

9246.8

10634.6

12023.1

$13412 \cdot 1$

$14801 \cdot 5$

16191.2

17581.0

$18971 \cdot 1$

$20361 \cdot 2$

$21751 \cdot 5$

$23141 \cdot 9$

$24532 \cdot 3$

25922.8

27313.3

28703.9

30094.5

31 485.2

32875.9

34266.6
$S$

$C A L / M O L \cdot K$

84.960

85.045

89.004

92.088

94.613

96.751

98.604

100.239

101.703

103.027

104.236

105.349

106.379

$107 \cdot 338$

108.235

109.078

109.873

110.625

111.338

112.016

112.663

113.282

113.873

114.441
( G-H298)/T

CAL/MOL $\cdot K$

$-84.960$

$-84.961$

$-85.500$

$-86.520$

$-87.665$

$-88.814$

$-89.925$

$-90.982$

$-91.982$

$-92.927$

$-93.820$

$-94.664$

$-95.465$

$-96.225$

$-96.948$

$-97.637$

$-98.295$

$-98.924$

$-99.527$

$-100.106$

$-100.662$

$-101.197$

$-101.713$

$-102.211$

FREQ 140 CM-1 MULT 1

FREQ 45 CM-1 MULT 1

FREQ 176 CM-1 MULT 1

MOLECULAR WEIGHT 485.847

SYMMETRY 2

MOMENTS $182.08 \quad 1753.3 \quad 1935.38$ IN AMU A2

GROUND STATE DEGENERACY 1 
$\operatorname{ThI}_{3}(g)$

T

$\begin{array}{ll}298 & 19.550 \\ 300 & 19.553 \\ 400 & 19.690 \\ 500 & 19.755 \\ 600 & 19.790 \\ 700 & 19.812 \\ 800 & 19.826 \\ 900 & 19.835 \\ 1000 & 19.842 \\ 1100 & 19.847 \\ 1200 & 19.851 \\ 1300 & 19.854 \\ 1400 & 19.857 \\ 1500 & 19.859 \\ 1600 & 19.860 \\ 1700 & 19.862 \\ 1800 & 19.863 \\ 1900 & 19.864 \\ 2000 & 19.864 \\ 2100 & 19.865 \\ 2200 & 19.866 \\ 2300 & 19.866 \\ 2400 & 19.867 \\ 2500 & 19.867\end{array}$

$H-H(B)$

CAL/MOL

5117.7

5153.9

7117.8

9089.7

11067.1

$13047 \cdot 3$

15029.2

$17012 \cdot 3$

18996.2

20980.7

22965.7

24951.0

26936.5

$28922 \cdot 3$

$30908 \cdot 2$

32894.3

$34880 \cdot 5$

36866.8

38853.2

40839.7

42826.2

44812.8

46799.4

48786.1
$S$

CAL/MOL $\cdot K$

102.733

102.854

108.501

112.902

116.508

119.560

122.207

124.542

126.633

128.524

130.251

131.840

133.312

134.682

135.963

137.167

$138 \cdot 303$

139.377

140.396

141.365

142.289

$143 \cdot 172$

144.817

144.828
( G-H298) /T

CAL/MOL.K

$-102.733$

$-102.734$

$-103.503$

$-104.959$

$-106.592$

$-108.232$

$-109.817$

$-111.326$

$-112.754$

$-114.103$

$-115.378$

$-116.584$

$-117.727$

$-118.812$

$-119.844$

$-120.828$

$-121.768$

$-122.667$

$-123.528$

$-124.354$

$-125.149$

$-125.913$

$-126.650$

$-127.361$

FREQ 144 CM-1 MULT 1

FREQ 53 CM-1 MULT 1

FREQ 170 CM-1 MULT 2

FREQ 48 CM-1 MULT 2

MOLECULAR WEIGHT 612.751

SYMMETRY 3

MOMENTS $1559.54 \quad 1559.94 \quad 2864.51$ IN AMU A2 GROUND STATE DEGENERACY 6 


$$
\operatorname{ThI}_{4}(c, 1)
$$

$\begin{array}{cc}T & \begin{array}{r}C P \\ C A L / M O L\end{array} \\ & \\ 298 & 30.27 \\ 300 & 30.30 \\ 400 & 31.32 \\ 500 & 31.96 \\ 600 & 32.45 \\ 700 & 32.87 \\ 800 & 33.25 \\ 839 & 33.39\end{array}$

$\begin{array}{cc}H T=11500 \text { CAL/MOL } \\ 839 & 42.00 \\ 900 & 42.00 \\ 1000 & 42.00 \\ 1100 & 42.00 \\ 1200 & 42.00 \\ 1300 & 42.00 \\ 1400 & 42.00 \\ 1500 & 42.00\end{array}$

$\mathrm{H}-\mathrm{H} 298$

CAL/MOL

0.
56.
3142.
6308
$9530^{\circ}$
12796.
$16102^{\circ}$
$17402^{\circ}$

$S T=13.707 \mathrm{CAL} / \mathrm{MOL} \cdot \mathrm{K}$

28902.

31464 .

35664 .

39864 .

44064 .

48264 .

52464 .

56664 .
$\stackrel{S}{\mathrm{CAL} / \mathrm{MOL} \cdot \mathrm{K}}$

61.00

$61 \cdot 19$

70.06

77.12

82.99

$88 \cdot 03$

$92 \cdot 44$

94.03
$(\mathrm{G}-\mathrm{H} 298) / \mathrm{T}$

CAL/MOL.K

$-61.00$

$-61 \cdot 00$

$-62 \cdot 20$

$-64.50$

$-67.11$

$-69 \cdot 75$

$-72 \cdot 31$

$-73.29$
AT $839 \mathrm{~K}$

$-73.29$

$-75.72$

$-79.44$

$-82.87$

$-86.04$

$-89.00$

$-91.76$

$-94.36$ 


\begin{tabular}{|c|c|c|c|}
\hline $\begin{array}{l}\text { U.S. DEPT. OF COMM. } \\
\text { BIBLIOGRAPHIC DATA } \\
\text { SHEET }\end{array}$ & $\begin{array}{l}\text { 1. IUIBI.ICATION OR RI:PORT NO. } \\
\text { NBSIR } 77-1300\end{array}$ & $\begin{array}{l}\text { 2. Ciov't Accession } \\
\text { No. }\end{array}$ & 3. Kecipient's Accession No. \\
\hline \multicolumn{3}{|c|}{$\begin{array}{l}\text { 4. TITI.I: ANII SUIITIII: } \\
\text { A COMPUTER-ASSISTED EVALUATION OF THE THERMOCHEMICAL } \\
\text { DATA OF THE COMPOUNDS OF THCRIUM }\end{array}$} & 6. Performing Organization Code \\
\hline 7. AUTHOR(S) D. D. W & an, R. H. Schumm and V. & Parker & 8. Performing Organ. Report No. \\
\hline \multicolumn{3}{|c|}{$\begin{array}{l}\text { 9. PERFORMING ORGANIZATION NAME AND ADDRESS } \\
\text { NATIONAL BUREAU OF STANDARDS } \\
\text { DEPARTMENT OF COMMERCE } \\
\text { WASHINGTON, D.C. } 20234\end{array}$} & $\begin{array}{l}\text { 10. Project/Task/Work Unit No. } \\
\text { 11. Contract/Grant No. }\end{array}$ \\
\hline \multirow{2}{*}{\multicolumn{3}{|c|}{ 12. Sponsoring Organization Name and Complete Address (Street, City, State, ZIP) }} & $\begin{array}{l}\text { 13. Type of Report \& Pesiod } \\
\text { Covered }\end{array}$ \\
\hline & & & 14. Sponsoring Agency Code \\
\hline
\end{tabular}

15. SUPPLEMENTARY NOTES

16. ABSTRACT (A 200-word or less factual summary of most significant information. If document includes a significant bibliography or literature survey, mention it here.)

Selected values are given for the thermochemical properties of the compounds of thorium. They are obtained from a computer-assisted least sums-least squares approach to the evaluation of thermodynamic data networks. The properties given, where data are available, are enthalpy of formation, Gibbs energy of formation, and entropy at $298.15 \mathrm{~K}\left(\Delta H f^{\circ}(298), \Delta G f^{\circ}(298)\right.$, and $\left.S^{\circ}(298)\right)$. The values are consistent with the CODATA Key Values for Thermodynamics. The reaction catalog from which this self consistent set of values is generated is given with a statistical analysis. Some thermal functions are also given, as well as detailed comments when necessary.

17. KEY WORDS (six to twelve entries; Alphabetical order; capitalize only the first letter of the first key word unless a proper name; separated by semicolons) Data evaluation; enthalpy; entropy; Gibbs energy;

thermochemical data networks; thermochemical tables; thorium compounds

18. AVAILABILITY

For Official Distribution. Do Not Release to NTIS

Order From Sup. of Doc., U.S. Government Printing Office Vashington, D.C. 20402 , SD Cat. No.C.13

[X] Order From Narional Technieal Infomation Service (NTS) Springfield, Virginia 22151

$$
\text { Q Unlimited }
$$$$
1
$$ 
